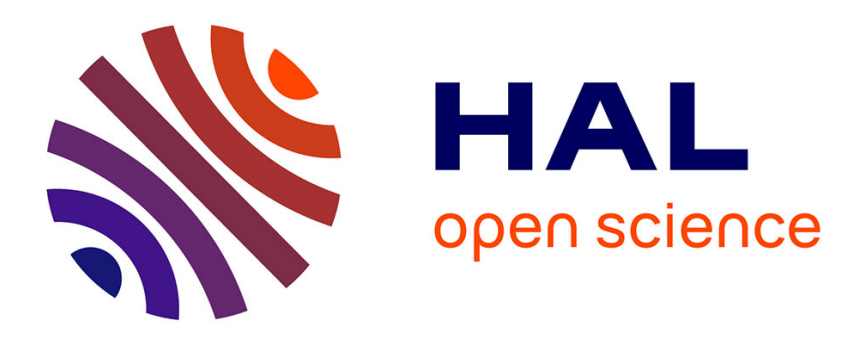

\title{
Mesures d'indépendance linéaire de logarithmes dans un groupe algébrique commutatif dans le cas rationnel
}

François Ballä̈

\section{To cite this version:}

François Ballaÿ. Mesures d'indépendance linéaire de logarithmes dans un groupe algébrique commutatif dans le cas rationnel. Dissertationes Mathematicae, 2019, 543, pp.1-78. 10.4064/dm781-5-2019 . hal-02428928

\section{HAL Id: hal-02428928 \\ https://hal.science/hal-02428928}

Submitted on 8 Feb 2020

HAL is a multi-disciplinary open access archive for the deposit and dissemination of scientific research documents, whether they are published or not. The documents may come from teaching and research institutions in France or abroad, or from public or private research centers.
L'archive ouverte pluridisciplinaire HAL, est destinée au dépôt et à la diffusion de documents scientifiques de niveau recherche, publiés ou non, émanant des établissements d'enseignement et de recherche français ou étrangers, des laboratoires publics ou privés. 


\title{
D I S S E R T A T I O N E S M A T H E M A T C A E
}

Online First version

EDITORIAL BOARD

\author{
ANDRZEJ BIA YNICKI-BIRULA, BOGDAN BOJARSKI, \\ JANUSZ GRABOWSKI, PIOTR GWIAZDA, \\ STANISEAW JANECZKO, PIOTR KOSZMIDER editor, \\ LUDOMIR NEWELSKI, WIESŁAW ŻELAZKO deputy editor
}

FRANÇOIS BALLA Ÿ

Mesures d'indépendance linéaire de logarithmes dans un groupe algébrique commutatif dans le cas rationnel 
Beijing International Center for Mathematical Research

Peking University

5 Yiheyuan Road Haidian District

Beijing 100871, China

E-mail: francois.ballay@bicmr.pku.edu.cn

Published by the Institute of Mathematics, Polish Academy of Sciences

Typeset using $\mathrm{T}_{\mathrm{E}} \mathrm{X}$ at the Institute

Abstracted/Indexed in: Mathematical Reviews, Zentralblatt MATH, Science Citation Index Expanded, Journal Citation Reports/Science Edition, Google Science, Scopus, EBSCO Discovery Service.

Available online at http://journals.impan.pl

(c) Copyright by Instytut Matematyczny PAN, Warszawa 2019

doi: 10.4064/dm781-5-2019

ISSN 0012-3862 


\section{Table des matières}

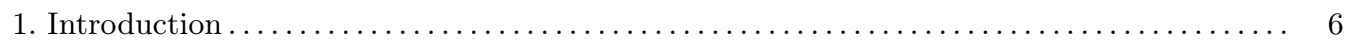

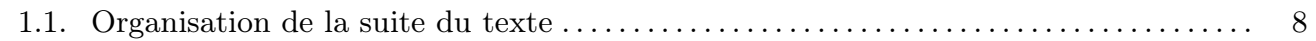

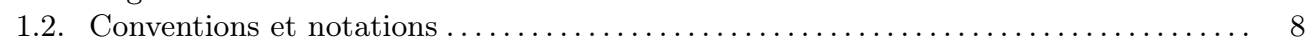

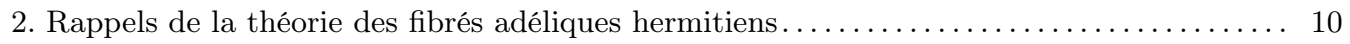

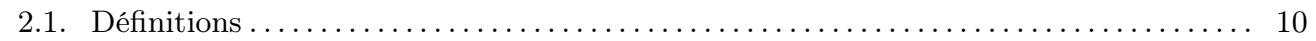

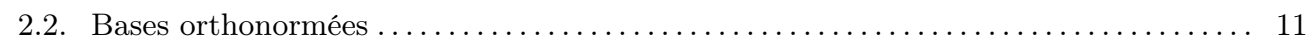

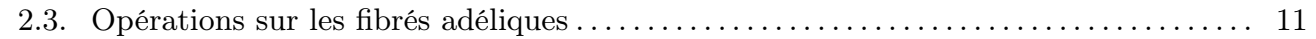

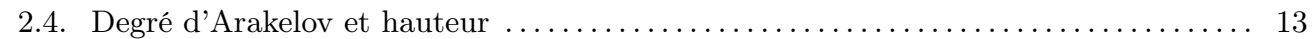

2.5. Hauteur d'un point et d'une application linéaire $\ldots \ldots \ldots \ldots \ldots \ldots \ldots \ldots \ldots \ldots$

2.6. Éléments de théorie des pentes adéliques $\ldots \ldots \ldots \ldots \ldots \ldots \ldots \ldots \ldots \ldots \ldots \ldots \ldots \ldots$

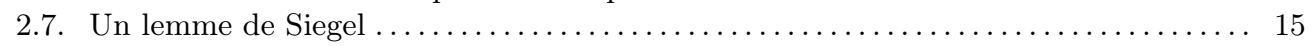

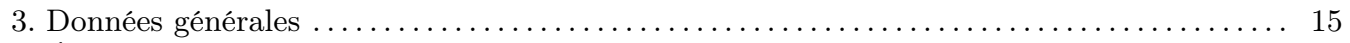

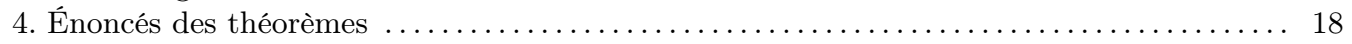

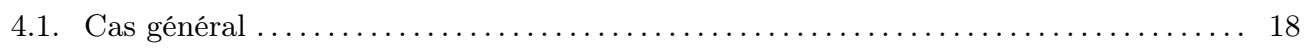

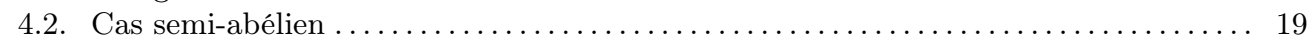

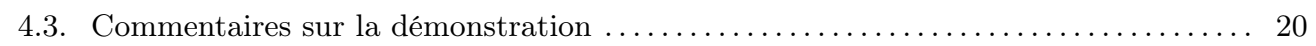

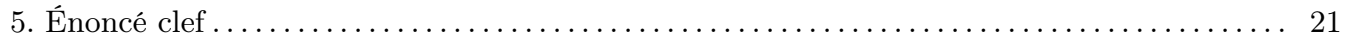

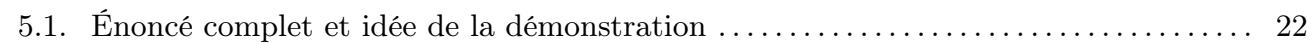

5.2. Groupe engendré par des sous-groupes algébriques $\ldots \ldots \ldots \ldots \ldots \ldots \ldots \ldots \ldots \ldots$

5.3. Degré d'un sous-groupe et hauteur de l'espace tangent $\ldots \ldots \ldots \ldots \ldots \ldots \ldots \ldots \ldots$

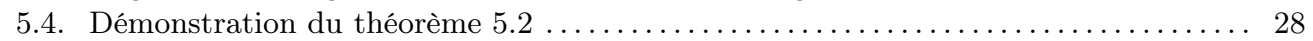

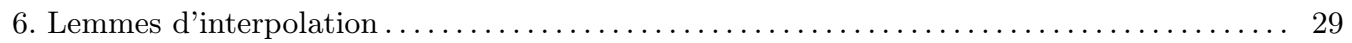

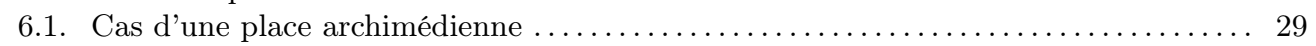

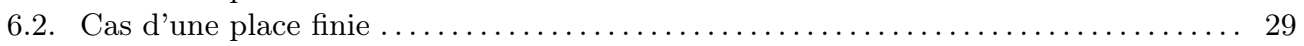

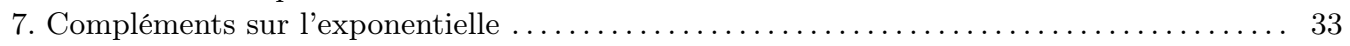

8. Démonstration dans le cas semi-abélien $\ldots \ldots \ldots \ldots \ldots \ldots \ldots \ldots \ldots \ldots \ldots \ldots \ldots \ldots \ldots \ldots \ldots$

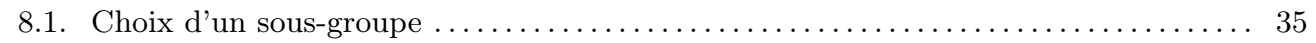

8.2. Fibré adélique hermitien des sections auxiliaires $\ldots \ldots \ldots \ldots \ldots \ldots \ldots \ldots \ldots \ldots . \ldots \ldots$

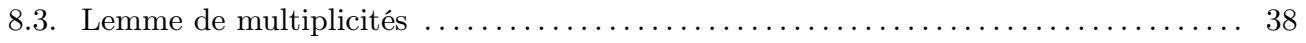

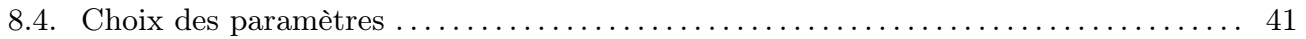

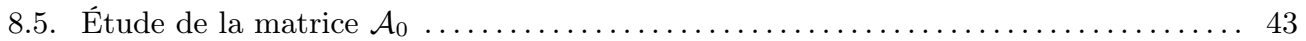

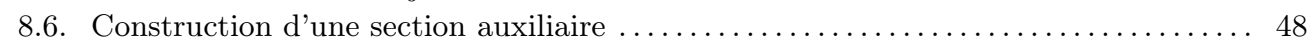

8.7. Construction d'un jet et premières estimations. ...................... 49

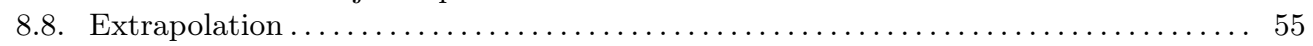

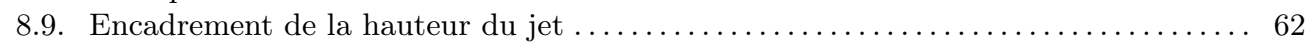

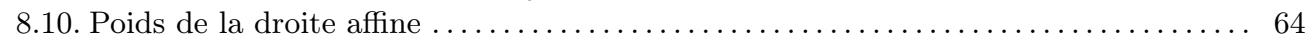

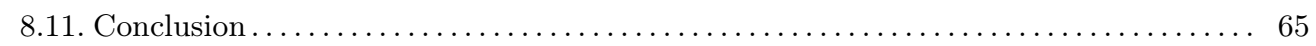

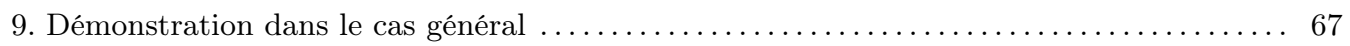

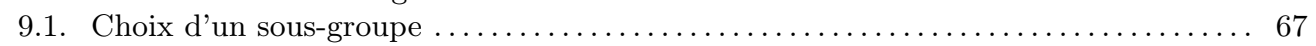

9.2. Fibré adélique hermitien des sections auxiliaires $\ldots \ldots \ldots \ldots \ldots \ldots \ldots \ldots \ldots \ldots .68$

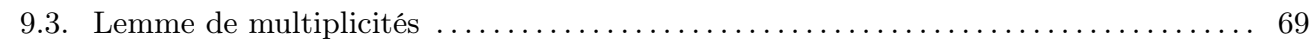




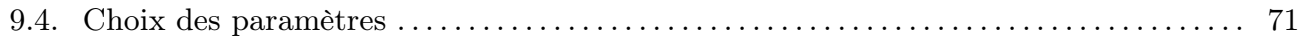

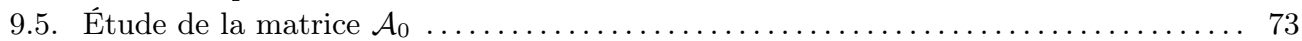

9.6. Construction d'une section auxiliaire $\ldots \ldots \ldots \ldots \ldots \ldots \ldots \ldots \ldots \ldots \ldots \ldots \ldots \ldots \ldots$

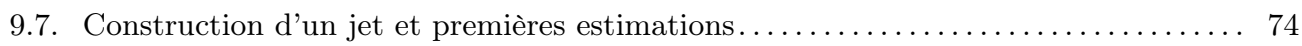

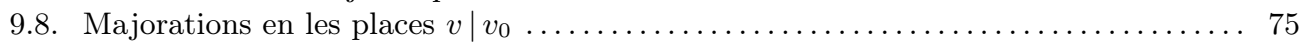

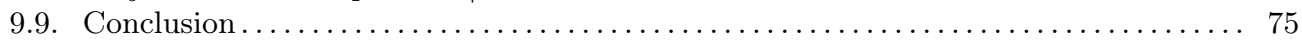

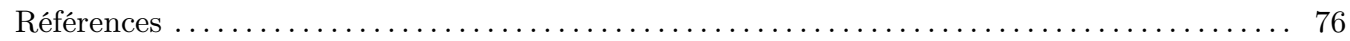




\begin{abstract}
We establish new measures of linear independence of logarithms on a commutative algebraic group. Let $G$ be a connected commutative algebraic group over $\overline{\mathbb{Q}}$ and let $t_{G}$ be the tangent space at the origin. We consider a vector $u \in t_{G} \otimes_{\bar{Q}} \mathbb{C}$ such that its image by the exponential map of the Lie group $G(\mathbb{C})$ is an algebraic point $\mathbf{p} \in G(\overline{\mathbb{Q}})$. Let $V$ be a hyperplane in $t_{G}$. We obtain lower bounds for the distance $d(u, V)$ between $u$ and $V \otimes_{\overline{\mathbb{Q}}} \mathbb{C}$ in the rational case, where $V=t_{H}$ is the tangent space at the origin of an algebraic connected subgroup of $G$. These lower bounds are the best currently known in terms of the height $h(\mathbf{p})$ of $\mathbf{p}$. They generalize measures of linear forms in logarithms previously obtained by Gaudron. Our approach is based on new arguments which allow us to exclude the so-called periodic case in the demonstration, by revisiting previous work of Bertrand and Philippon. Our proofs also rely on tools from Bost's slope theory of hermitian vector bundles. Moreover, we present ultrametric analogues of our results, and we deal with the case where $V=t_{H}$ is a linear subspace of any dimension.
\end{abstract}

Remerciements. Je remercie mes deux directeurs de thèse, Huayi Chen et Éric Gaudron, pour leur soutien et leurs encouragements lors de l'élaboration de ce travail. Je suis très reconnaissant envers Éric Gaudron pour m'avoir suggéré ce thème de recherche, ainsi que pour ses précieux conseils et sa lecture attentive de versions précédentes de ce manuscrit. Je remercie également le rapporteur anonyme pour son important travail de relecture, ainsi que pour ses suggestions qui ont permis d'améliorer la clarté du texte.

2010 Mathematics Subject Classification: Primary 11J86; Secondary 11J61, 14G40.

Key words and phrases: linear forms in logarithms, commutative algebraic group, rational case, periodic case, Baker's method, hermitian vector bundles, Siegel's lemma, $p$-adic interpolation. Received 25 January 2018; revised 23 May 2019.

Published online*. 


\section{Introduction}

Dans ce texte, nous établissons de nouvelles mesures d'indépendance linéaire de logarithmes dans un groupe algébrique commutatif. Nous donnerons des mesures archimédiennes ainsi que leurs analogues ultramétriques. Pour simplifier, nous considérons uniquement le cas archimédien dans cette introduction. Soit $G$ un groupe algébrique connexe et commutatif sur $\overline{\mathbb{Q}}$. L'espace tangent à l'origine $t_{G}$ de $G$ est un $\overline{\mathbb{Q}}$-espace vectoriel de dimension $g:=\operatorname{dim} G$. Soit $V$ un sous-espace vectoriel de $t_{G}$. Fixons un plongement de $\overline{\mathbb{Q}}$ dans $\mathbb{C}$ et notons $t_{G}(\mathbb{C})=t_{G} \otimes_{\overline{\mathbb{Q}}} \mathbb{C}$. Le groupe de Lie $G(\mathbb{C})$ est muni d'une application exponentielle exp: $t_{G}(\mathbb{C}) \rightarrow G(\mathbb{C})$, qui est surjective. Considérons un point $\mathbf{p} \in G(\overline{\mathbb{Q}})$ et un logarithme $u \in t_{G}(\mathbb{C})$ de $\mathbf{p}$ (i.e. un vecteur $u \in t_{G}(\mathbb{C})$ tel que $\exp (u)=\mathbf{p}$ ). Dans le cas où le vecteur $u$ n'appartient pas à $V \otimes_{\bar{Q}} \mathbb{C}$, une mesure d'indépendance linéaire de logarithmes est une minoration de la distance $d(u, V)$ séparant $u$ et $V \otimes_{\overline{\mathbb{Q}}} \mathbb{C}$ en fonction d'invariants liés aux données de départ, notamment la hauteur $h(V)$ de $V$ (définition 2.6) et la hauteur de Weil logarithmique $h(\mathbf{p})$ du point $\mathbf{p}$ (relative à un plongement fixé de $G$ dans un espace projectif). Nous nous plaçons dans le cas dit rationnel, où $V$ est l'espace tangent à l'origine d'un sous-groupe algébrique connexe $H$ de $G$. Dans ce contexte, Gaudron a établi les meilleures minorations connues à ce jour en termes de la hauteur de $\mathbf{p}$ [21]. Cet article combine la méthode de Baker, revisitée par Philippon et Waldschmidt [34, 35], à des minorations de la taille de sous-schémas formels dues à Bost [8]. Cependant, les théorèmes de [21] imposent systématiquement l'hypothèse suivante sur le point $\mathbf{p}$ : pour tout sous-groupe algébrique $G^{\prime}$ de $G$ tel que $t_{G^{\prime}}+V \neq t_{G}$ et pour tout entier naturel $k$ non nul, on a $k \mathbf{p} \notin G^{\prime}(\overline{\mathbb{Q}})$. L'objectif principal de ce travail est de retrouver les mesures d'indépendance linéaires de logarithmes de [21] en supprimant cette hypothèse technique. Celle-ci peut en effet s'avérer très contraignante, et exclut par exemple le cas où $\mathbf{p}$ est un point de torsion. Elle est précisément introduite pour exclure de la démonstration le cas dit périodique, qui pose de sérieux problèmes lorsque l'on cherche à adapter la méthode de Baker au cas général d'un groupe algébrique commutatif quelconque. En particulier, il empêche d'appliquer les méthodes classiques à deux étapes clefs de la démonstration, à savoir la majoration du rang d'un système d'équations linéaire et l'application d'un lemme de multiplicités. L'inconvénient est que ces deux points font intervenir certains sous-groupes de $G$, appelés sous-groupes obstructeurs, et que les arguments classiques sont mis en échec si un multiple de $\mathbf{p}$ appartient à ces sous groupes. Dans leurs articles [34] et [35], Philippon et Waldschmidt ont mis en place une méthode très astucieuse pour intégrer le cas périodique dans les démonstrations de minorations de formes linéaires de logarithmes, au moyen d'une extrapolation sur les dérivations inspirée par les travaux 
de Gel'fond. Cette méthode est devenue très classique en théorie des formes linéaires de logarithmes depuis; elle est par exemple appliquée dans les travaux de Hirata-Kohno [28, 29], David [14, David et Hirata-Kohno [15, Gaudron [19, 24], ou encore Bosser et Gaudron [6] (dans le langage plus moderne des fibrés vectoriels adéliques pour les deux dernières références). Cet outil a néanmoins l'inconvénient d'être assez lourd à mettre en place, car il nécessite un choix de paramètres spécifique au cas périodique. De façon plus problématique, il s'avère que si l'on intègre une extrapolation sur les dérivations dans la démonstration de [21] (qui repose sur un raisonnement par l'absurde), l'on n'aboutit pas à une contradiction, et il est impossible de conclure. C'est la raison pour laquelle les théorèmes de [21] contiennent l'hypothèse $« t_{G^{\prime}}+V \neq t_{G} \Rightarrow \forall k \in \mathbb{N} \backslash\{0\}, k \mathbf{p} \notin G^{\prime}(\overline{\mathbb{Q}}) »$.

Dans ce travail, nous proposons une nouvelle approche pour traiter le cas périodique, qui nous permet de retrouver tous les théorèmes de [21] en remplaçant l'hypothèse sur $\mathbf{p}$ ci-dessus par : pour tout sous-groupe algébrique $G^{\prime}$ de $G$ tel que $t_{G^{\prime}}+V \neq t_{G}$, le vecteur $u$ n'appartient pas à $t_{G^{\prime}}(\mathbb{C})$. Notre hypothèse est bien plus faible que celle de [21]; par exemple, quand $V$ est un hyperplan (qui est la situation généralisant le cas historique de la théorie, traitant d'une forme linéaire de logarithme), cette condition signifie simplement que le point $u$ n'appartient pas à $V \otimes_{\overline{\mathbb{Q}}} \mathbb{C}$. Par ailleurs, dans le cas où $\operatorname{dim} V<g-1$ (qui correspond à la minoration simultanée de plusieurs formes linéaires de logarithmes), cette hypothèse est courante dans la littérature, et figure par exemple dans les travaux de Hirata-Kohno [29] et de Philippon et Waldschmidt [34]. Nous démontrerons le résultat suivant. Rappelons que $V=t_{H}$ est l'espace tangent à l'origine d'un sous-groupe algébrique connexe $H$ de $G$.

THÉORÈmE 1.1. Il existe une constante $q \geq 1$, indépendante de $h(V)$ et de $h(\mathbf{p})$, ayant la propriété suivante. Soit $t=\operatorname{codim}_{t_{G}} V$. Supposons que pour tout sous-groupe algébrique connexe $G^{\prime}$ de $G$ tel que $t_{G^{\prime}}+V \neq t_{G}$, on ait $u \notin t_{G^{\prime}}(\mathbb{C})$. Alors $u \notin V \otimes_{\overline{\mathbb{Q}}} \mathbb{C}$ et

$$
\log d(u, V) \geq-q \max \{1, h(V)\}^{1+(g+1) / t} \max \{1, h(\mathbf{p})\}^{g / t} .
$$

Nous énoncerons des théorèmes plus précis au paragraphe 4 , et nous en donnerons également des analogues ultramétriques. Nos résultats généralisent tous les théorèmes de [21], en remplaçant l'hypothèse $« t_{G^{\prime}}+V \neq t_{G} \Rightarrow \forall k \in \mathbb{N} \backslash\{0\}, k \mathbf{p} \notin G^{\prime}(\overline{\mathbb{Q}}) »$ par celle du théorème 1.1. En particulier, lorsque $V$ est un hyperplan, nous supposons seulement que $u$ n'appartient pas à $V \otimes_{\overline{\mathbb{Q}}} \mathbb{C}$. Nous améliorons également les mesures obtenues en termes du degré d'un corps de nombres de définition de $G$ et de $H$.

L'argument clef de notre approche repose sur la démonstration d'une variante d'un résultat de Bertrand et Philippon 3 . Notons $\Omega_{G} \subset t_{G}(\mathbb{C})$ le réseau des périodes de $G$, défini comme le noyau de l'exponentielle exp. Le corollaire 2 de [3] permet de comparer le degré $\operatorname{deg}\left(G^{\prime}\right)$ d'un sous-groupe algébrique $G^{\prime}$ de $G$ avec la distance $d\left(\omega, t_{G^{\prime}}(\mathbb{C})\right)$ séparant un élément $\omega \in \Omega_{G} \backslash t_{G^{\prime}}(\mathbb{C})$ de l'espace tangent $t_{G^{\prime}}(\mathbb{C})$ de $G^{\prime}(\mathbb{C})$. Nous commencerons par établir une généralisation du corollaire 2 de Bertrand et Philippon [3], qui s'applique à un point $\omega \in\left(t_{G} \backslash t_{G^{\prime}}\right)(\mathbb{C})$ qui n'est plus nécessairement une période (lemme 5.4 page 23 ). Nous démontrerons ensuite le théorème suivant, conséquence du théorème 5.1 .

THÉORÈME 1.2. Il existe une constante $q_{2} \geq 1$ ayant la propriété suivante. Soit $\widetilde{G}$ un sous-groupe algébrique connexe de $G$ tel que $t_{\widetilde{G}}+V \neq t_{G}$ et soit $S$ un entier naturel non 
nul. Supposons que pour tout sous-groupe algébrique connexe $G^{\prime}$ de $G$ tel que $t_{G^{\prime}}+V \neq t_{G}$, on ait $u \notin t_{G^{\prime}}(\mathbb{C})$. Alors on a l'implication

$$
d(u, V)<\frac{1}{S \operatorname{deg} \widetilde{G} \exp \left(q_{2} \max \{1, h(V)\}\right)} \Longrightarrow \forall s \in\{1, \ldots, S\}, s \mathbf{p} \notin \widetilde{G}(\overline{\mathbb{Q}}) .
$$

Nous appliquerons ensuite ce théorème de façon systématique, pour montrer que si la distance $d(u, V)$ ne satisfait pas la minoration voulue, alors les sous-groupes obstructeurs de $G$ ne contiennent pas de petits multiples de p. Nous évitons ainsi les difficultés techniques liées au cas périodique, sans avoir recours à une extrapolation sur les dérivations. La démonstration du théorème 1.2 nécessite une étude minutieuse des relations entre le degré d'un sous-groupe et la hauteur de son espace tangent, au moyen d'un « dévissage » grâce à une décomposition de Chevalley, et repose sur des résultats intermédiaires sur les liens entre les sous-groupes algébriques de $G$ et leurs algèbres de Lie. Une autre caractéristique importante de notre démonstration du théorème 1.1 est d'adopter, pour la première fois dans le contexte général d'un groupe algébrique commutatif quelconque, le schéma de démonstration novateur de l'article [24] de Gaudron (traitant du cas d'un groupe linéaire). Celui-ci intègre des outils de la théorie des fibrés vectoriels hermitiens de Bost [7] à la méthode de Baker. Ce formalisme permet de clarifier l'argumentation en faisant ressortir de façon intrinsèque les invariants associés aux données de départ. Nous nous affranchissons par exemple des contraintes techniques liées aux choix de bases particulières pour $V$ (qui nécessitaient l'emploi d'un lemme de Siegel dans [21]). Signalons enfin que dans le cas ultramétrique, nous remplaçons également une hypothèse faite sur la norme de $u$ dans l'article 21] de Gaudron par la condition naturelle que $u$ appartienne au disque de convergence de l'application exponentielle. Cette amélioration repose sur l'utilisation d'un cas très particulier d'un lemme d'interpolation dû à Robba [36. corollaire 6.3 , page 33 .

1.1. Organisation de la suite du texte. Le paragraphe 2 regroupe les rappels de la théorie des fibrés vectoriels hermitiens dont nous aurons besoin. Nous préciserons les données du problème et les énoncés des théorèmes principaux aux paragraphes 3 et 4 La démonstration du théorème 1.2 constitue l'objet du $\$ 5$ Le paragraphe 6 regroupe quant à lui des lemmes d'interpolation (archimédien et ultramétrique) qui seront mis en œuvre lors de la démonstration du théorème 1.1 ( $\$ \$ 7,9$ ).

\subsection{Conventions et notations}

Notations. Pour tout nombre réel $a$, on note $[a]$ la partie entière de $a$ et $\log _{+} a=$ $\log \max \{1, a\}$, où $\log$ désigne le logarithme népérien. Si $\mathbf{x}=\left(x_{1}, \ldots, x_{n}\right) \in \mathbb{N}^{n}$ alors $\mathbf{x} !:=x_{1} ! \cdots x_{n}$ ! et $|\mathbf{x}|:=x_{1}+\cdots+x_{n}$. Si $\mathbf{x}=\left(x_{1}, \ldots, x_{n}\right)$ est un $n$-uplet d'éléments d'un corps $K$ et si $\mathbf{j}=\left(j_{1}, \ldots, j_{n}\right) \in \mathbb{N}^{n}$, on note $\mathbf{x}^{\mathbf{j}}:=x_{1}^{j_{1}} \cdots x_{n}^{j_{n}}$ et

$$
\mathcal{D}_{\mathbf{x}}=x_{1} \frac{\partial}{\partial z_{1}}+\cdots+x_{n} \frac{\partial}{\partial z_{n}}
$$

l'opérateur différentiel $K$-linéaire sur l'algèbre des polynômes $K\left[z_{1}, \ldots, z_{n}\right]$, de coordonnées $\mathbf{x}$ dans la base $\left(\frac{\partial}{\partial z_{1}}, \ldots, \frac{\partial}{\partial z_{n}}\right)$. Enfin, si $\mathbf{b}=\left(b_{1}, \ldots, b_{n}\right)$ est une base de $K^{n}$, on 
pose

$$
\mathcal{D}_{\mathbf{b}}^{\mathbf{j}}=\mathcal{D}_{b_{1}}^{j_{1}} \circ \cdots \circ \mathcal{D}_{b_{n}}^{j_{n}} .
$$

Normes et valeurs absolues. Si $K$ est un corps de nombres, on note $\Sigma_{K, f}$ l'ensemble des places finies de $K, \Sigma_{K, \infty}$ l'ensemble de ses places infinies, et $\Sigma_{K}=\Sigma_{K, f} \cup \Sigma_{K, \infty}$ l'ensemble des places de $K$. Si $v \in \Sigma_{K}$, on note $K_{v}$ le complété de $K$ en $v, \mathscr{O}_{K_{v}}$ l'anneau des entiers de $K_{v}$, et $\mathbb{C}_{v}$ le complété d'une clôture algébrique de $K_{v}$. Pour toute place $v \in \Sigma_{K}$, on note $|\cdot|_{v}$ la valeur absolue sur $K$ étendant la valeur absolue $|\cdot|_{v}$ sur $\mathbb{Q}$ : $|p|_{v}=p^{-1}$ si $v$ est une place finie au-dessus de $p$ et la restriction de $|\cdot|_{v}$ à $\mathbb{Q}$ est la valeur absolue usuelle si $v$ est archimédienne. On désigne par $\mathbb{Q}_{v}$ le corps $\mathbb{Q}_{p}, \mathbb{R}$ ou $\mathbb{C}$ selon le caractère $p$-adique, réel ou complexe non réel de $v$. On peut alors énoncer la formule du produit : pour tout $x \in K \backslash\{0\}$, on a

$$
\prod_{v \in \Sigma_{K}}|x|_{v}^{\left[K_{v}: \mathbb{Q}_{v}\right]}=1
$$

Soit $n$ un entier naturel non nul et soit $v \in \Sigma_{K}$. Si $\left(z_{1}, \ldots, z_{n}\right)$ est un vecteur de $\mathbb{C}_{v}^{n}$, on pose

$$
\left|\left(z_{1}, \ldots, z_{n}\right)\right|_{v}:= \begin{cases}\left(\sum_{i=1}^{n}\left|z_{i}\right|_{v}^{2}\right)^{1 / 2} & \text { si } v \text { est archimédienne } \\ \max \left\{\left|z_{1}\right|_{v}, \ldots,\left|z_{n}\right|_{v}\right\} & \text { sinon. }\end{cases}
$$

Hauteur d'un point projectif. La formule du produit permet de définir la notion de hauteur d'un point projectif : si $x=\left(x_{0}: \ldots: x_{n}\right) \in \mathbb{P}_{K}^{n}(\overline{\mathbb{Q}})$ est un point fermé, dont les coordonnées appartiennent à une extension finie $L$ de $K$, on note $h(x)$ sa hauteur de Weil (logarithmique et absolue), définie par

$$
h(x)=\sum_{v \in \Sigma_{L}} \frac{\left[L_{v}: \mathbb{Q}_{v}\right]}{[L: \mathbb{Q}]} \log \max \left\{\left|x_{0}\right|_{v}, \ldots,\left|x_{n}\right|_{v}\right\},
$$

où $\left(x_{0}, \ldots, x_{n}\right)$ est un représentant quelconque de $x$. La hauteur d'un point fermé projectif ne dépend pas du choix du corps $L$. On dispose ainsi d'une notion de hauteur pour tout point $x \in \mathbb{P}^{n}(\bar{K})$ (où $\bar{K}$ désigne une clôture algébrique de $K$ ). Si $X$ est une variété quasiprojective et si $\iota: X \hookrightarrow \mathbb{P}_{K}^{n}$ est un plongement fixé de $X$ dans un espace projectif, on notera $h(x)=h(\iota(x))$ pour tout point $x \in X(\bar{K})$.

Polynôme de Hilbert-Samuel et degré d'une variété quasi-projective. Si $V$ est une sous variété quasi-projective d'un produit d'espaces projectifs $\mathbb{P}=\mathbb{P}_{\overline{\mathbb{Q}}}^{N_{0}} \times \cdots \times \mathbb{P}_{\overline{\mathbb{Q}}}^{N_{\ell}}$, on note $\operatorname{deg} V$ le degré de l'adhérence de Zariski $\bar{V}$ de $V$ dans $\mathbb{P}$ relatif au faisceau $\mathscr{O}_{\mathbb{P}}(1, \ldots, 1)$. On note $H\left(V ; X_{0}, \ldots, X_{\ell}\right)$ le polynôme de Hilbert-Samuel de $\bar{V}$, et $\mathscr{H}\left(V ; X_{0}, \ldots, X_{\ell}\right)$ la partie homogène de plus haut degré $(=\operatorname{dim} V)$ de $H\left(V ; X_{0}, \ldots, X_{\ell}\right)$ multipliée par $(\operatorname{dim} V)$ !. Les coefficients de $\mathscr{H}\left(V ; X_{0}, \ldots, X_{\ell}\right)$ sont des entiers de somme égale à $\mathscr{H}(V ; 1, \ldots, 1)=$ $\operatorname{deg} V$. Le polynôme $\mathscr{H}\left(V ; X_{0}, \ldots, X_{\ell}\right)$ vérifie les propriétés suivantes (voir [20, §5.3]) :

— Si $V=V_{0} \times \cdots \times V_{\ell}$ est un sous-schéma produit de $\mathbb{P}$, alors

$$
\mathscr{H}\left(V ; X_{0}, \ldots, X_{\ell}\right)=(\operatorname{dim} V) ! \prod_{i=0}^{\ell} \frac{\mathscr{H}\left(V_{i} ; X_{i}\right)}{\left(\operatorname{dim} V_{i}\right) !} .
$$


- Si $i \in\{0, \ldots, \ell\}$ et si $\pi$ désigne la projection de $V$ sur les $i+1$ premiers facteurs de $\mathbb{P}$, alors pour tout $\left(x_{0}, \ldots, x_{\ell}\right) \in\left[1,+\infty\left[^{\ell+1}\right.\right.$,

$$
\left(\begin{array}{c}
\operatorname{dim} V \\
\operatorname{dim} \pi(V)
\end{array}\right) \mathscr{H}\left(\pi(V) ; x_{0}, \ldots, x_{i}\right) \leq \mathscr{H}\left(V ; x_{0}, \ldots, x_{\ell}\right) .
$$

\section{Rappels de la théorie des fibrés adéliques hermitiens}

Soit $K$ un corps de nombres. L'objectif de ce paragraphe est de rappeler quelques éléments de la théorie des pentes des fibrés vectoriels hermitiens sur Spec $\mathscr{O}_{K}$, introduite par Bost [7] dans les années 90. Nous nous appuierons sur les travaux de Gaudron, qui a généralisé cette théorie dans un cadre adélique dans l'article [22].

\subsection{Définitions}

DÉFinition 2.1. Un fibré (vectoriel) adélique sur $K$ est la donnée $\bar{E}=\left(E,\|\cdot\|_{v}\right)_{v \in \Sigma_{K}}$ d'un $K$-espace vectoriel $E$ et, pour chaque place $v$ de $K$, d'une norme $\|\cdot\|_{v}$ sur $E \otimes_{K} \mathbb{C}_{v}$ satisfaisant les conditions suivantes :

(1) il existe une base $\left(e_{1}, \ldots, e_{n}\right)$ de $E$ sur $K$ et une partie finie $\mathcal{S}$ de $\Sigma_{K, f}$ telle que $\left\|a_{1} e_{1}+\cdots+a_{n} e_{n}\right\|_{v}=\max \left(\left|a_{1}\right|_{v}, \ldots,\left|a_{n}\right|_{v}\right)$ pour toute place $v \in \Sigma_{K, f} \backslash \mathcal{S}$ et pour tout vecteur $\left(a_{1}, \ldots, a_{n}\right) \in \mathbb{C}_{v}^{n}$;

(2) pour toute place $v \in \Sigma_{K}$, la norme $\|\cdot\|_{v}$ est invariante sous l'action du groupe de Galois $\operatorname{Gal}\left(\mathbb{C}_{v} / K_{v}\right):$ si $\left(s_{1}, \ldots, s_{n}\right)$ désigne une base de $E \otimes_{K} K_{v}$ sur $K_{v}$, alors

$$
\left\|\sigma\left(a_{1}\right) s_{1}+\cdots+\sigma\left(a_{n}\right) s_{n}\right\|_{v}=\left\|a_{1} s_{1}+\ldots+a_{n} s_{n}\right\|_{v}
$$

quels que soient $\sigma \in \operatorname{Gal}\left(\mathbb{C}_{v} / K_{v}\right)$ et $\left(a_{1}, \ldots, a_{n}\right) \in \mathbb{C}_{v}^{n}$;

(3) si $v \in \Sigma_{f}$, alors la norme $\|\cdot\|_{v}$ est ultramétrique :

$$
\left\|s+s^{\prime}\right\|_{v} \leq \max \left(\|s\|_{v},\left\|s^{\prime}\right\|_{v}\right) \quad \forall s, s^{\prime} \in E \otimes_{K} \mathbb{C}_{v} .
$$

DÉFinition 2.2. Un fibré adélique $\left(E,\left(\|\cdot\|_{E, v}\right)_{v \in \Sigma_{K}}\right)$ est dit pur si pour toute place $v$ de $K$ et tout $x$ dans $E$, la norme $\|x\|_{E, v}$ appartient à $\left|K_{v}\right|_{v}$. Un fibré adélique est dit hermitien s'il est pur et si les normes aux places archimédiennes de $K$ sont hermitiennes.

À l'exception des paragraphes 2.5 et 2.7 . nous considérons exclusivement des fibrés adéliques hermitiens dans la suite. Les fibrés adéliques hermitiens sur $K$ sont exactement les fibrés vectoriels hermitiens sur Spec $\mathscr{O}_{K}$ au sens de Bost (voir [24, proposition 3.10]). Signalons que la notion d'《espace adélique rigide», introduite par Gaudron et Rémond [27, généralise celle de fibré adélique hermitien pour des espace vectoriels définis sur un sous-corps de $\overline{\mathbb{Q}}$.

EXemple 2.3. Soit $n$ un entier naturel non nul. On munit l'espace vectoriel $K^{n}$ d'une structure naturelle de fibré adélique hermitien en considérant la norme $|\cdot|_{v}$ sur $\mathbb{C}_{v}^{n}$ pour chaque place $v$ de $K$. Nous dirons dans ce cas que $K^{n}$ est muni de la structure triviale de fibré adélique. Soit $E$ est un espace vectoriel de dimension $n$ et soit $\left(e_{1}, \ldots, e_{n}\right)$ une base de $E$. Cette base permet d'identifier $E$ à $K^{n}$, et fournit ainsi une structure de fibré 
adélique hermitien $\left(E,\left(\|\cdot\|_{v}\right)_{v \in \Sigma_{K}}\right)$ à $E$. Pour chaque place $v$ de $\Sigma_{K}$ la norme $\|\cdot\|_{E, v}$ obtenue est donnée par

$$
\forall\left(z_{1}, \ldots, z_{n}\right) \in \mathbb{C}_{v}^{n}, \quad\left\|z_{1} e_{1}+\cdots+z_{n} e_{n}\right\|_{v}=\left|\left(z_{1}, \ldots, z_{n}\right)\right|_{v} .
$$

2.2. Bases orthonormées. Soit $\bar{E}=\left(E,\left(\|\cdot\|_{E, v}\right)_{v \in \Sigma_{K}}\right)$ un fibré adélique hermitien de dimension $r \geq 1$.

Définition 2.4. Soit $v$ une place de $K$. Une base $\left(e_{1}, \ldots, e_{r}\right)$ de $E \otimes_{K} \mathbb{C}_{v}$ est dite orthonormée pour la norme $\|\cdot\|_{E, v}$ si $\left\|e_{i}\right\|_{E, v}=1$ pour tout $i \in\{1, \ldots, r\}$ et si

$$
\left\|a_{1} e_{1}+\cdots+a_{r} e_{r}\right\|_{E, v}=\left|\left(a_{1}, \ldots, a_{r}\right)\right|_{v}
$$

pour toute famille $\left(a_{i}\right)_{1 \leq i \leq r}$ d'éléments de $\mathbb{C}_{v}$.

Le procédé d'orthonormalisation de Gram-Schmidt permet de construire une base orthonormée de $E \otimes_{K} \mathbb{C}_{v}$ pour toute place archimédienne $v$ de $K$. Cette propriété n'est pas vérifiée dans le cas ultramétrique. Cependant, on dispose de l'analogue asymptotique suivant.

Proposition 2.5 ([24, corollaire du lemme 3.8]). Soit $v$ une place ultramétrique de $K$ et soit $\alpha \in\left[0,1\left[\right.\right.$ un nombre réel. Soit $L_{v}$ une extension complète de $K$ (pour la valeur absolue $\left.|\cdot|_{v}\right)$. Alors il existe une base $\mathbf{e}=\left(e_{1}, \ldots, e_{r}\right)$ de $E \otimes_{K} L_{v}$ formée de vecteurs de norme égale 1 et telle que

$$
\alpha \max _{1 \leq i \leq r}\left|a_{i}\right|_{v} \leq\left\|a_{1} e_{1}+\cdots+a_{r} e_{r}\right\|_{E, v} \leq \max _{1 \leq i \leq r}\left|a_{i}\right|_{v}
$$

pour toute famille $\left(a_{i}\right)_{1 \leq i \leq r}$ d'éléments de $L_{v}$.

Signalons que le lemme 3.8 de 24 est plus général, car il donne un résultat valable pour un corps complet muni d'une valeur absolue ultramétrique.

2.3. Opérations sur les fibrés adéliques. Considérons deux fibrés adéliques hermitiens $\bar{E}=\left(E,\left(\|\cdot\|_{E, v}\right)_{v}\right), \bar{F}=\left(F,\left(\|\cdot\|_{F, v}\right)_{v}\right)$ sur $K$.

Sous-fibré et quotient. Nous dirons que $\bar{F}$ est un sous-fibré adélique de $\bar{E}$ si $F \subset E$ et si la structure de fibré adélique de $F$ correspond à celle induite par celle de $E$ : pour chaque place $v$ de $K$ la norme $\|\cdot\|_{F, v}$ correspond à la restriction de $\|\cdot\|_{E, v}$ à $F \otimes_{K} \mathbb{C}_{v}$. Si $\bar{F}$ est un sous-fibré adélique de $\bar{E}$, nous noterons $\bar{F} \subset \bar{E}$. Dans ce cas, on peut construire le fibré adélique $\bar{E} / \bar{F}=\left(E / F,\left(\|\cdot\|_{E / F, v}\right)_{v}\right)$, en considérant les normes quotient : si $v$ est une place de $K$ et si $\bar{x}$ est un élément de $(E / F) \otimes_{K} \mathbb{C}_{v}$, alors

$$
\|\bar{x}\|_{E / F, v}=\inf \left\{\|x\|_{E, v} \mid x \in E \otimes_{K} \mathbb{C}_{v}, \bar{x}=x \bmod F \otimes_{K} \mathbb{C}_{v}\right\} .
$$

Fibré dual. On définit le fibré adélique hermitien dual de $\bar{E}$, noté $\bar{E}^{\vee}$, donné par les normes d'opérateurs

$$
\|\varphi\|_{v}=\sup \left\{\frac{|\varphi(x)|_{v}}{\|x\|_{E, v}} \mid x \in\left(E \otimes \mathbb{C}_{v}\right) \backslash\{0\}\right\}
$$

où $\varphi$ est un élément de $\left(E \otimes \mathbb{C}_{v}\right)^{\vee}$. De façon plus générale, on peut munir le $K$-espace vectoriel $\operatorname{Hom}_{K}(E, F)$ d'une structure de fibré adélique hermitien en considérant pour 
chaque place $v$ de $K$ la norme d'opérateur sur $\operatorname{Hom}_{K}(E, F) \otimes_{K} \mathbb{C}_{v}$ définie par

$$
\|\varphi\|_{v}=\sup \left\{\frac{\|\varphi(x)\|_{F, v}}{\|x\|_{E, v}} \mid x \in\left(E \otimes_{K} \mathbb{C}_{v}\right) \backslash\{0\}\right\} .
$$

Somme directe. On définit le fibré adélique hermitien $\bar{E} \oplus \bar{F}$ d'espace sous-jacent $E \oplus F$ et de normes

$$
\|(x, y)\|_{E \oplus F, v}= \begin{cases}\left(\|x\|_{\bar{E}, v}^{2}+\|y\|_{\bar{F}, v}^{2}\right)^{1 / 2} & \text { si } v \text { est archimédienne, } \\ \max \left\{\|x\|_{\bar{E}, v},\|y\|_{\bar{F}, v}\right\} & \text { si } v \text { est ultramétrique, }\end{cases}
$$

pour tous $x \in E \otimes \mathbb{C}_{v}$ et $y \in F \otimes \mathbb{C}_{v}$.

Produit tensoriel. L'espace vectoriel $E \otimes_{K} F$ est isomorphe à $\operatorname{Hom}_{K}\left(E^{\vee}, F\right)$. On pourrait donc munir $E \otimes_{K} F$ de la structure de fibré adélique induite par celle de $\operatorname{Hom}_{K}\left(E^{\vee}, F\right)$. Cependant, contrairement au cas de la somme directe, cette construction ne fait pas de $E \otimes_{K} F$ un fibré adélique hermitien en général (voir [22, page 43]). Pour pallier à ce problème, il suffit de choisir des normes convenables en les places archimédiennes, comme dans [24, §3.3] : soit $v \in \Sigma_{K}$ une place archimédienne et soient $\left(e_{1}, \ldots, e_{n}\right)$ et $\left(f_{1}, \ldots, f_{m}\right)$ des bases orthonormées des espaces vectoriels $E \otimes_{K} \mathbb{C}_{v}$ et $F \otimes_{K} \mathbb{C}_{v}$ respectivement. On munit alors l'espace vectoriel $E \otimes_{K} F \otimes_{K} \mathbb{C}_{v}$ de l'unique norme $\|\cdot\|_{E \otimes_{K} F, v}$ rendant la base $\left(e_{i} \otimes f_{j}\right)_{1 \leq i \leq n, 1 \leq j \leq m}$ orthonormée :

$$
\forall\left(x_{i, j}\right) \in \mathbb{C}_{v}^{n m}, \quad\left\|\sum_{i, j} x_{i, j} e_{i} \otimes f_{j}\right\|_{E \otimes_{K} F, v}:=\left|\left(x_{i, j}\right)_{i, j}\right|_{v} .
$$

En les places ultramétriques, on considère la norme $\|\cdot\|_{E \otimes_{K} F, v}$ sur $E \otimes_{K} F \otimes_{K} \mathbb{C}_{v}$ induite par l'isomorphisme $E \otimes_{K} F \simeq \operatorname{Hom}_{K}\left(E^{\vee}, F\right)$. On note alors $\bar{E} \otimes_{K} \bar{F}=\left(E \otimes_{K} F\right.$, $\left.\left(\|\cdot\|_{E \otimes_{K} F, v}\right)_{v}\right)$ le fibré adélique obtenu, qui est hermitien.

Puissance symétrique et puissance extérieure. Si $\ell$ est un entier naturel, nous noterons $S^{\ell}(E)$ sa puissance symétrique $\ell$-ième de l'espace vectoriel $E$. C'est un quotient de $E^{\otimes \ell}$, que l'on peut donc munir de la structure de fibré adélique induite par celle de $\bar{E}^{\otimes \ell}$. Nous noterons $S^{\ell}(\bar{E})$ le fibré adélique correspondant. On peut expliciter la norme $\|\cdot\|_{S^{\ell} \bar{E}, v}$ pour une place archimédienne $v$ de $K$. Si $\left(e_{1}, \ldots, e_{r}\right)$ est une base orthonormée de $E \otimes_{K} \mathbb{C}_{v}$, alors la base $\left\{e_{1}^{i_{1}} \cdots e_{r}^{i_{r}}\left|\mathbf{i}=\left(i_{j}\right)_{1 \leq j \leq r} \in \mathbb{N}^{r},\right| \mathbf{i} \mid=\ell\right\}$ de $S^{\ell}(E)$ est orthogonale et la norme de ses éléments vérifie

$$
\left\|e_{1}^{i_{1}} \cdots e_{r}^{i_{r}}\right\|_{S^{\ell} \bar{E}, v}=\left(\frac{\mathbf{i} !}{\ell !}\right)^{1 / 2}
$$

(voir [22, page 46]). Si $\ell \leq r=\operatorname{dim} E$, la $\ell$-ième puissance extérieure $\bigwedge^{\ell} E$ de $E$ est également un quotient de $E^{\otimes \ell}$. Pour toute place ultramétrique $v$ de $K$, on munit l'espace vectoriel $\left(\bigwedge^{\ell} E\right) \otimes_{K} \mathbb{C}_{v}$ de la norme quotient $\|\cdot\|_{\Lambda^{\ell} E, v}$ induite par $\|\cdot\|_{E \otimes \ell, v}$. Si $v$ est une place archimédienne de $K$ et si $\left(e_{1}, \ldots, e_{r}\right)$ est une base orthonormée de $E \otimes_{K} \mathbb{C}_{v}$ pour la norme $\|\cdot\|_{E, v}$, on note $\|\cdot\|_{\Lambda^{\ell} E, v}$ l'unique norme $\operatorname{sur}\left(\bigwedge^{\ell} E\right) \otimes_{K} \mathbb{C}_{v}$ rendant la base $\left\{e_{i_{1}} \wedge \cdots \wedge e_{i_{\ell}} \mid 1 \leq i_{1}<\cdots<i_{\ell} \leq r\right\}$ orthonormée. Remarquons que contrairement au cas des places ultramétriques, cette norme n'est pas exactement la norme quotient de $\|\cdot\|_{E \otimes \ell, v}$ (un facteur $\sqrt{\ell !}$ différencie ces normes, voir [26, page 572]). Ces définitions confèrent une structure de fibré vectoriel adélique à $\bigwedge^{\ell} E$, et l'on note $\bigwedge^{\ell} \bar{E}$ le fibré 
adélique ainsi construit. Les fibrés vectoriels adéliques $S^{\ell} \bar{E}$ et $\bigwedge^{\ell} \bar{E}$ sont hermitiens. En particulier, le déterminant $\operatorname{det} E:=\bigwedge^{\operatorname{dim} E} E$ est muni d'une structure de fibré adélique hermitien.

2.4. Degré d'Arakelov et hauteur. Soit $\bar{E}=\left(E,\left(\|\cdot\|_{E, v}\right)_{v \in \Sigma_{K}}\right)$ un fibré adélique hermitien sur $K$.

DÉfinition 2.6. Si $\bar{E}$ est non nul, le degré d'Arakelov de $\bar{E}$ est le nombre réel

$$
\widehat{\operatorname{deg}}_{\mathrm{n}} \bar{E}:=-\sum_{v \in \Sigma_{K}} \frac{\left[K_{v}: \mathbb{Q}_{v}\right]}{[K: \mathbb{Q}]} \log \left\|e_{1} \wedge \cdots \wedge e_{\operatorname{dim} E}\right\|_{\operatorname{det} E, v},
$$

où $\left(e_{1}, \ldots, e_{\operatorname{dim} E}\right)$ est une $K$-base de $E$. Si $E=\{0\}$, on pose $\widehat{\operatorname{deg}}_{n}(\bar{E}):=0$. La hauteur $h(\bar{E})$ de $\bar{E}$ est alors définie par $h(\bar{E}):=-\widehat{\operatorname{deg}}_{n}(\bar{E})$.

Par la formule du produit, cette définition ne dépend pas du choix de la base de $E$. La notation $\widehat{\operatorname{deg}}_{n}$ souligne le fait que le degré d'Arakelov est normalisé par le degré du corps de nombres $[K: \mathbb{Q}]$.

Les énoncés suivants sont des cas particuliers des propositions 4.19, 4.22 et 4.23 de [22].

Proposition 2.7.

(1) Si $\bar{F} \subset \bar{E}$ est un sous-fibré adélique de $\bar{E}$, alors

$$
h(\bar{E} / \bar{F})=h(\bar{E})-h(\bar{F}) .
$$

(2) Si $\bar{E}_{1}$ et $\bar{E}_{2}$ sont deux sous-fibré adéliques de $\bar{E}$, alors

$$
h\left(\bar{E}_{1} \oplus \bar{E}_{2}\right)=h\left(\bar{E}_{1}\right)+h\left(\bar{E}_{2}\right) .
$$

2.5. Hauteur d'un point et d'une application linéaire. Soit $\bar{E}$ un fibré adélique sur $K$ (non nécessairement hermitien). Si $K^{\prime}$ est une extension finie de $K$, alors pour toute place $v^{\prime} \in \Sigma_{K^{\prime}}$ au-dessus d'une place $v$ de $K$, on considère la norme $\|\cdot\|_{E, v^{\prime}}$ définie de la façon suivante. Si $\sigma^{\prime}: K^{\prime} \hookrightarrow \mathbb{C}_{v}$ et $\sigma: K \hookrightarrow \mathbb{C}_{v}$ sont des plongements associés respectivement à $v^{\prime}$ et $v$, alors pour toutes familles finies d'éléments $e_{i} \in E, x_{i} \in K^{\prime}$, $y_{i} \in \mathbb{C}_{v}$, on pose

$$
\left\|\sum_{i}\left(e_{i} \otimes_{K} x_{i}\right) \otimes_{\sigma^{\prime}} y_{i}\right\|_{E, v^{\prime}}=\left\|\sum_{i} e_{i} \otimes_{\sigma}\left(\sigma^{\prime}\left(x_{i}\right) y_{i}\right)\right\|_{E, v} .
$$

Définition 2.8. Soit $K^{\prime}$ une extension finie de $K$ et soit $s \in E \otimes_{K} K^{\prime}$ un vecteur non nul. La hauteur (logarithmique, absolue) de $s$ est définie par

$$
h_{\bar{E}}(s)=\sum_{v \in \Sigma_{K^{\prime}}} \frac{\left[K_{v}^{\prime}: \mathbb{Q}_{v}\right]}{\left[K^{\prime}: \mathbb{Q}\right]} \log \|s\|_{E, v} .
$$

La hauteur de $s$ sera notée plus simplement $h(s)$ si aucune confusion n'est possible. Par la formule du produit, $h(\lambda s)=h(s)$ pour tout $\lambda \in\left(K^{\prime}\right)^{\times}$. Cette définition est également invariante par extension finie de corps. Cette dernière propriété permet de définir la hauteur de tout élément non nul de $E \otimes_{K} \overline{\mathbb{Q}}$. 
Si $\bar{F}$ est un fibré adélique sur $K$ et si $\varphi: E \rightarrow F$ est une application $K$-linéaire, nous définissons la hauteur de $\varphi$ relativement à $\bar{E}, \bar{F}$ par

$$
h(\varphi):=\sum_{v \in \Sigma_{K}} \frac{\left[K_{v}: \mathbb{Q}_{v}\right]}{[K: \mathbb{Q}]} \log \|\varphi\|_{v},
$$

où $\|\varphi\|_{v}$ désigne la norme d'opérateur de l'application $E \otimes_{K} \mathbb{C}_{v} \rightarrow F \otimes_{K} \mathbb{C}_{v}$ induite par $\varphi$.

2.6. Éléments de théorie des pentes adéliques. Soit $\bar{E}$ un fibré adélique hermitien sur $K$ de dimension supérieure ou égale à 1 .

DÉFInition 2.9. La pente d'Arakelov normalisée de $\bar{E}$ est

$$
\widehat{\mu}_{\mathrm{n}}(\bar{E})=\frac{\widehat{\operatorname{deg}}_{\mathrm{n}} \bar{E}}{\operatorname{dim} E} .
$$

Lemme 2.10 ([22, lemme 6.3]). Soit $\bar{F}$ un fibré adélique hermitien sur $K$ et soit $\varphi: E \rightarrow F$ un isomorphisme de K-espaces vectoriels. On a

$$
\widehat{\mu}_{\mathrm{n}}(\bar{E}) \leq h(\varphi)+\widehat{\mu}_{\mathrm{n}}(\bar{F}) .
$$

D'après la proposition 5.3 de [22, l'intersection de l'ensemble

$$
\Delta(\bar{E})=\left\{\left(\operatorname{dim} F, \widehat{\operatorname{deg}}_{\mathrm{n}}(\bar{F})\right) \mid \bar{F} \subset \bar{E}\right\}
$$

avec $\{(x, y) \mid y \geq t\} \subset \mathbb{R}^{2}$ est finie pour tout nombre réel $t$. L'enveloppe convexe de $\Delta(\bar{E})$ est délimitée par une fonction concave et affine par morceaux $\mathcal{P}_{\bar{E}}:[0, \operatorname{dim} E] \rightarrow \mathbb{R}$. Pour tout entier $i \in\{1, \ldots, \operatorname{dim} E\}$, on définit la $i$-ème pente de $\bar{E}$, notée $\widehat{\mu}_{i}(\bar{E})$, en posant

$$
\widehat{\mu}_{i}(\bar{E})=\mathcal{P}_{\bar{E}}(i)-\mathcal{P}_{\bar{E}}(i-1)
$$

(voir [22, définition 5.9]). Nous allons rappeler les propriétés vérifiées par ces pentes dont nous aurons besoin, qui sont démontrées au paragraphe 5.2 de $[22]$.

Proposition 2.11. On $a$ :

(1) $\sum_{i=1}^{\operatorname{dim} E} \widehat{\mu}_{i}(\bar{E})=\widehat{\operatorname{deg}}_{\mathrm{n}}(\bar{E})$;

(2) $\widehat{\mu}_{\operatorname{dim} E}(\bar{E}) \leq \cdots \leq \widehat{\mu}_{1}(\bar{E})$;

(3) $\widehat{\mu}_{1}(\bar{E})=\max \left\{\widehat{\mu}_{\mathrm{n}}(\bar{F}) \mid \bar{F} \subset \bar{E}, F \neq\{0\}\right\}$;

(4) pour tout $i \in\{1, \ldots, \operatorname{dim} E\}$,

$$
\widehat{\mu}_{i}(\bar{E})=-\widehat{\mu}_{\operatorname{dim} E-i+1}\left(\bar{E}^{\vee}\right) .
$$

Dans la suite, nous noterons $\widehat{\mu}_{\max }(\bar{E})$ la quantité $\widehat{\mu}_{1}(\bar{E})=\max \left\{\widehat{\mu}_{\mathrm{n}}(\bar{F}) \mid \bar{F} \subset \bar{E}\right.$, $F \neq\{0\}\}$, appelée pente maximale de $\bar{E}$.

EXEMPLE 2.12. Si $E$ est muni d'une structure de fibré adélique triviale comme à l'exemple 2.3 alors toutes ses pentes successives $\widehat{\mu}_{1}(\bar{E}), \ldots, \widehat{\mu}_{\operatorname{dim} E}(\bar{E})$ sont nulles. En particulier, $\widehat{\mu}_{\max }(\bar{E})=0$.

Dans la suite du texte, nous serons amenés à comparer la pente maximale d'un fibré adélique hermitien avec celles de ses puissances symétriques, au moyen du résultat suivant. Proposition 2.13 ([24, proposition 3.16]). Pour tout entier $\ell \geq 1$,

$$
\widehat{\mu}_{\max }\left(S^{\ell} \bar{E}\right) \leq \ell\left(\widehat{\mu}_{\max }(\bar{E})+2 \log (\operatorname{dim} E)\right) .
$$


2.7. Un lemme de Siegel. Un lemme de Siegel est un outil récurrent dans les démonstrations de transcendance. Il permet de construire un vecteur non nul $s$ de petite 《 taille »dans un $K$-espace vectoriel $E$, de sorte que $s$ soit solution d'un système d'équations linéaires, voire d'un système d'inéquations (on parle alors de lemme de Siegel approché). Dans le langage des fibrés adéliques, le mot «taille » désigne la hauteur de $s$. Quand $E$ est l'espace des sections globales d'un fibré inversible (par exemple, un espace de polynômes), ce vecteur est couramment appelé section auxiliaire. De nombreuses versions sont apparues dans la littérature depuis l'énoncé originel de Siegel en 1929 (voir par exemple [4, [23], 25]). Dans leurs premières versions, les majorations de la hauteur de $s$ faisaient intervenir le discriminant du corps de nombres $K$. Il est possible de s'affranchir de cette dépendance en construisant la section $s$ dans l'espace $E \otimes_{K} \overline{\mathbb{Q}}$, comme l'ont montré Roy et Thunder [37]. (voir aussi Zhang [43]); on parle alors de lemme de Siegel absolu. Pour un historique plus complet sur ces avancées, nous renvoyons à l'article de Gaudron [24, §4], dont le « lemme de Siegel approché absolu » que nous présentons est issu.

Soient $\bar{E}_{1}=\left(E_{1},\left(\|\cdot\|_{E_{1}, v}\right)_{v \in \Sigma_{K}}\right)$ et $\bar{E}_{2}=\left(E_{2},\left(\|\cdot\|_{E_{2}, v}\right)_{v \in \Sigma_{K}}\right)$ deux fibrés vectoriels adéliques hermitiens sur $K$. Soit $v_{0} \in \Sigma_{K}$ une place de $K$ et soit $A_{0}: E_{1} \otimes_{K} \mathbb{C}_{v_{0}} \rightarrow$ $E_{2} \otimes_{K} \mathbb{C}_{v_{0}}$ une application $\mathbb{C}_{v_{0}}$-linéaire. Étant donné un nombre réel $\alpha>0$, on considère la norme «tordue » $\|\cdot\|_{\alpha, v_{0}}$ sur $E_{1} \otimes_{K} \mathbb{C}_{v_{0}}$ définie par

$$
\forall x \in E_{1} \otimes_{K} \mathbb{C}_{v_{0}}, \quad\|x\|_{\alpha, v_{0}}:= \begin{cases}\max \left\{\|x\|_{E_{1}, v_{0}}, \alpha\left\|A_{0} x\right\|_{E_{2}, v_{0}}\right\} & \text { si } v_{0} \text { est finie, } \\ \left(\|x\|_{E_{1}, v_{0}}^{2}+\alpha^{2}\left\|A_{0} x\right\|_{E_{2}, v_{0}}^{2}\right)^{1 / 2} & \text { sinon. }\end{cases}
$$

On construit alors un nouveau fibré adélique $\bar{E}_{\alpha}=\left(E_{1},\left(\|\cdot\|_{\alpha, v}\right)_{v \in \Sigma_{K}}\right)$, en posant $\|\cdot\|_{\alpha, v}=$ $\|\cdot\|_{E_{1}, v}$ pour toute place $v \neq v_{0}$ de $K$. Nous pouvons alors énoncer un cas particulier du lemme de [24, page 24].

LEMME 2.14. En conservant les notations ci-dessus, il existe un vecteur non nul $x$ de $E \otimes_{K} \bar{K}$ tel que

$$
h_{\bar{E}_{\alpha}}(x) \leq \frac{\operatorname{rg} A_{0}}{\operatorname{dim} E} \frac{\left[K_{v_{0}}: \mathbb{Q}_{v_{0}}\right]}{[K: \mathbb{Q}]} \log _{+}\left(2 \alpha\left\|A_{0}\right\|_{v_{0}}\right)+\frac{1}{2} \log (\operatorname{dim} E)-\widehat{\mu}_{\mathrm{n}}(\bar{E}) .
$$

Démonstration. L'énoncé est un cas particulier du lemme de Siegel approché de [24, page 24], appliqué aux fibrés adéliques $\bar{E}=\bar{E}_{1}$ et $\bar{F}=\left(E_{2},\left(\alpha\|\cdot\|_{E_{2}, v}\right)_{v \in \Sigma_{K}}\right)$ et à l'ensemble $S$ des plongements associés à $v_{0}$. Il est important de remarquer que l'article 24] n'impose pas de condition de pureté dans la définition d'un fibré adélique hermitien (en particulier, les fibrés adéliques $\bar{F}$ et $\bar{E}_{\alpha}$ sont hermitiens au sens de [24]).

Convention. Dans la suite du texte, un fibré adélique hermitien $\bar{E}$ sera simplement désigné par son espace-vectoriel sous-jacent $E$ afin de ne pas alourdir les notations.

\section{Données générales}

Considérons des groupes algébriques connexes commutatifs $G_{1}, \ldots, G_{n}$ sur $\overline{\mathbb{Q}}$ et posons $G=G_{1} \times_{\overline{\mathbb{Q}}} \cdots \times_{\overline{\mathbb{Q}}} G_{n}$. La loi d'addition de ces groupes sera notée + . Pour tout 
$i \in\{1, \ldots, n\}$, on note $t_{G_{i}}$ l'espace tangent à l'origine de $G_{i}$ (qui est un $\overline{\mathbb{Q}}$-espace vectoriel de dimension $\left.g_{i}:=\operatorname{dim} G_{i}\right)$. Considérons un corps de nombres $K$ sur lequel les groupes $G_{1}, \ldots, G_{n}$ sont définis. Pour éviter toute confusion, nous noterons $t_{G_{1}}(K), \ldots, t_{G_{n}}(K)$ les espaces tangents en l'origine des groupes $G_{1}, \ldots, G_{n}$ quand ils seront considérés comme des $K$-espaces vectoriels. Dans la suite du texte, un sous-groupe algébrique de $G$ sera considéré comme défini sur $\overline{\mathbb{Q}}$, sauf précision supplémentaire.

Plongements projectifs. Soit $i \in\{1, \ldots, n\}$. On considère un plongement $\phi_{i}: G_{i} \hookrightarrow$ $\mathbb{P}_{K}^{N_{i}}$ du type de ceux construits par Serre dans [39, Appendice II]. Quitte à effectuer un changement de coordonnées, on peut supposer que $\phi_{i}$ envoie l'élément neutre de $G_{i}$ sur $(1: 0: \cdots: 0)$. Nous noterons $\phi$ le plongement produit :

$$
\phi:=\phi_{1} \times \cdots \times \phi_{n}: G_{1} \times_{K} \cdots \times_{K} G_{n} \hookrightarrow \mathbb{P}_{K}^{N_{1}} \times \cdots \times \mathbb{P}_{K}^{N_{n}} .
$$

Application exponentielle. Soit $v$ une place quelconque de $K$ et soit $i \in\{1, \ldots, n\}$. On dispose d'une application exponentielle $\exp _{v, i}$ du groupe de Lie $v$-adique $G_{i}\left(\mathbb{C}_{v}\right)$, définie sur un voisinage ouvert de l'origine dans $t_{G_{i}}\left(\mathbb{C}_{v}\right):=t_{G_{i}}(K) \otimes_{K} \mathbb{C}_{v}$ (voir [9, Chapitre III, §4.3]). Si la place $v$ est archimédienne, cette application se prolonge en une fonction $\mathbb{C}_{v}$-analytique sur tout $t_{G_{i}}\left(\mathbb{C}_{v}\right)$, et définit une application surjective $\exp _{v, i}$ : $t_{G_{i}}\left(\mathbb{C}_{v}\right) \rightarrow G_{i}\left(\mathbb{C}_{v}\right)$. Lorsque la place $v$ est ultramétrique, il existe un voisinage ouvert $\mathscr{U}_{v, i}$ de l'origine dans $t_{G_{i}}\left(\mathbb{C}_{v}\right)$ tel que la restriction de $\exp _{v, i}$ à $\mathscr{U}_{v, i}$ réalise un difféomorphisme sur son image. Afin d'uniformiser les notations, nous noterons $\mathscr{U}_{v, i}=t_{G_{i}}\left(\mathbb{C}_{v}\right)$ si $v$ est archimédienne $\left({ }^{1}\right)$. Par abus de notation, on confondra souvent $\exp _{v, i}$ avec $\phi_{i} \circ \exp _{v, i}$.

Il existe des fonctions $\varphi_{v, i, 0}, \ldots, \varphi_{v, i, N_{i}}$, analytiques de $\mathscr{U}_{v, i} \subseteq t_{G_{i}}\left(\mathbb{C}_{v}\right)$ dans $\mathbb{C}_{v}$, et vérifiant :

- $\left(\varphi_{v, i, 0}(0), \ldots, \varphi_{v, i, N_{i}}(0)\right)=(1,0, \ldots, 0)$;

- les fonctions $\varphi_{v, i, j}, 0 \leq j \leq N_{i}$, sont sans zéro commun sur $\mathscr{U}_{v, i}$;

- pour tout $z \in \mathscr{U}_{v, i}$,

$$
\exp _{v, i}(z)=\left(\varphi_{v, i, 0}(z): \cdots: \varphi_{v, i, N_{i}}(z)\right) .
$$

Enfin, posons $\mathscr{U}_{v}:=\mathscr{U}_{v, 1} \times \cdots \times \mathscr{U}_{v, n}$ et

$$
\exp _{v}=\exp _{v, 1} \times \cdots \times \exp _{v, n}: \mathscr{U}_{v} \rightarrow G_{1}\left(\mathbb{C}_{v}\right) \times \cdots \times G_{n}\left(\mathbb{C}_{v}\right) .
$$

On confondra souvent $\exp _{v}$ avec $\phi \circ \exp _{v}$.

Choix d'une structure de fibré adélique hermitien. D'après [21, page 224], il existe un entier $\widetilde{m} \in \mathbb{N} \backslash\{0\}$ et des modèles lisses $\mathfrak{G}_{i} \rightarrow \operatorname{Spec} \mathscr{O}_{K}[1 / \widetilde{m}]$ de $G_{i}$ pour tout $i \in\{1, \ldots, n\}$, avec $\mathscr{O}_{K}[1 / \widetilde{m}]$ principal (voir aussi [18, lemme I.5.1]). On note $\mathfrak{G}=$ $\mathfrak{G}_{1} \times \cdots \times \mathfrak{G}_{n}$. Pour tout $i \in\{1, \ldots, n\}, t_{\mathfrak{G}_{i}}$ est un module projectif de type fini sur l'anneau principal $\mathscr{O}_{K}[1 / \widetilde{m}]$, donc il est libre de dimension $g_{i}$.

Soit $i \in\{1, \ldots, n\}$. On se donne une structure de fibré adélique hermitien sur $t_{G_{i}}(K)$, que l'on note $\left(t_{G_{i}}(K),\left(\|\cdot\|_{v}\right)_{v \in \Sigma_{K}}\right)$. On suppose que pour toute place ultramétrique $v$

$\left.{ }^{1}\right)$ Dans ce cas l'exponentielle ne réalise pas forcément un difféomorphisme de $\mathscr{U}_{v, i}$ sur son image, contrairement au cas ultramétrique. 
de $K$ ne divisant pas $\widetilde{m}$, la norme $\|\cdot\|_{v}$ satisfait la condition suivante : il existe une base $\left(e_{v, 1}, \ldots, e_{v, g_{i}}\right)$ du $\mathscr{O}_{K}[1 / \widetilde{m}]$-module libre $t_{\mathfrak{G}_{i}}$ telle que pour tout $\left(z_{1}, \ldots, z_{g_{i}}\right) \in \mathbb{C}_{v}^{g_{i}}$,

$$
\left\|z_{1} e_{v, 1}+\cdots+z_{g_{i}} e_{v, g_{i}}\right\|_{v}=\max _{1 \leq j \leq g_{i}}\left|z_{j}\right|_{v} .
$$

Pour toute place $v \nmid \widetilde{m}$, nous pouvons considérer le $\mathscr{O}_{K_{v}}$-module $t_{\mathfrak{G}_{i}} \otimes \mathscr{O}_{K_{v}}$, et notre choix de normes assure que $t_{\mathfrak{G}_{i}} \otimes \mathscr{O}_{K_{v}}=\left\{z \in t_{G_{i}}\left(K_{v}\right) \mid\|z\|_{v} \leq 1\right\}$. Cette propriété nous sera utile au paragraphe 8.7.1. Ces choix fournissent une structure de fibré adélique hermitien sur $t_{G}(K)=t_{G_{1}}(K) \oplus \cdots \oplus t_{G_{n}}(K)$, que l'on note $\left(t_{G}(K),\left(\|\cdot\|_{v}\right)_{v \in \Sigma_{K}}\right)$.

Dans la suite, on fixe une place $v_{0} \in \Sigma_{K}$. Si $v_{0}$ est une place ultramétrique au-dessus d'un nombre premier $p_{0}$, nous supposerons de plus que la norme $\|\cdot\|_{v_{0}}$ est définie de la façon suivante. Quitte à agrandir l'entier $\widetilde{m}$, il existe une base $\mathbf{f}_{i}=\left(f_{i, 1}, \ldots, f_{i, g_{i}}\right)$ de $t_{\mathfrak{G}_{i}}$ telle que le polydisque

$$
D_{i}\left(0, r_{p_{0}}\right):=\left\{z_{1} f_{i, 1}+\cdots+\left.z_{g_{i}} f_{i, g_{i}} \in t_{G_{i}}\left(\mathbb{C}_{v_{0}}\right)\left|\max _{1 \leq j \leq g_{i}}\right| z_{j}\right|_{v_{0}}<r_{p_{0}}\right\}
$$

soit inclus dans $\mathscr{U}_{v_{0}, i}$, où $r_{p_{0}}=\left|p_{0}\right|_{v_{0}}^{1 /\left(p_{0}-1\right)}=p_{0}^{-1 /\left(p_{0}-1\right)}$. De plus, l'exponentielle $\exp _{v_{0}, i}$ admet un développement en série entière à coefficients dans $\mathscr{O}_{K_{v_{0}}}$ au voisinage de 0 , et son domaine de convergence strict contient le polydisque $D_{i}\left(0, r_{p_{0}}\right)$. Pour tout $j \in\left\{0, \ldots, N_{i}\right\}$, la fonction $\varphi_{v_{0}, i, j}$ vérifie : il existe une suite $\left(a_{v_{0}, j, \mathbf{n}}\right)_{\mathbf{n} \in \mathbb{N}^{g_{i}}}$ dans $\mathscr{O}_{K_{v_{0}}}$ telle que pour tout $z=z_{1} f_{i, 1}+\ldots+z_{g_{i}} f_{i, g_{i}} \in D_{i}\left(0, r_{p_{0}}\right)$, on ait

$$
\varphi_{v_{0}, i, j}(z)=\sum_{\mathbf{n} \in \mathbb{N}^{g_{i}}} \frac{a_{v_{0}, j, \mathbf{n}}}{\mathbf{n} !} \mathbf{z}^{\mathbf{n}}, \quad \text { où } \mathbf{z}=\left(z_{1}, \ldots, z_{g_{i}}\right) .
$$

Pour plus de détails sur cette construction, on pourra se référer à [21, page 224]. Par concaténation des bases $\mathbf{f}_{i}$ des $K$-espaces vectoriels $t_{G_{i}}(K)$, on construit une base $\mathbf{e}=$ $\left(e_{1}, \ldots, e_{g}\right)$ de $t_{G}(K)$. On définit alors $\|\cdot\|_{v_{0}}$ comme étant l'unique norme sur $t_{G}\left(\mathbb{C}_{v_{0}}\right)$ rendant la base e orthonormée. On note

$$
D\left(0, r_{p_{0}}\right):=\left\{z_{1} e_{1}+\cdots+\left.z_{g} e_{g} \in t_{G}\left(\mathbb{C}_{v_{0}}\right)\left|\max _{1 \leq j \leq g}\right| z_{j}\right|_{v_{0}}<r_{p_{0}}\right\} .
$$

Données du problème. Dans la suite, on fixe un vecteur $u=\left(u_{1}, \ldots, u_{n}\right) \in \mathscr{U}_{v_{0}}=$ $\mathscr{U}_{v_{0}, 1} \times \cdots \times \mathscr{U}_{v_{0}, n}$. Pour tout $i \in\{1, \ldots, n\}$, on note $\mathbf{p}_{i}=\exp _{v_{0}, i}\left(u_{i}\right)$ et on suppose que $\mathbf{p}_{i} \in G_{i}(K)$. On pose $\mathbf{p}=\left(\mathbf{p}_{1}, \ldots, \mathbf{p}_{n}\right) \in G(K)$. Si $v_{0}$ est ultramétrique, on suppose de plus que $u \in D\left(0, r_{p_{0}}\right)$. Nous notons $d(\cdot, \cdot)$ la distance associée à la norme $\|\cdot\|_{v_{0}}$ sur $t_{G}\left(\mathbb{C}_{v_{0}}\right)$. On fixe un sous-espace vectoriel $V$ de $t_{G}(K)$ de codimension $1 \leq t \leq g$, et on suppose que $V=t_{H}(K)$ est l'algèbre de Lie d'un sous-groupe algébrique connexe $H$ de $G$ défini sur $K$. Dans la suite, nous cherchons à minorer la distance $d\left(u, V \otimes_{K} \mathbb{C}_{v_{0}}\right)=$ $\inf \left\{\|u-x\|_{v_{0}} \mid x \in V \otimes_{K} \mathbb{C}_{v_{0}}\right\}$. Pour simplifier, nous noterons cette distance $d(u, V)$. On fixe également une extension de la place $v_{0}$ à $\overline{\mathbb{Q}}$, et pour tout sous-groupe algébrique $G^{\prime}$ de $G$, on note $t_{G^{\prime}}\left(\mathbb{C}_{v_{0}}\right)=t_{G^{\prime}} \otimes_{\overline{\mathbb{Q}}} \mathbb{C}_{v_{0}}$.

Convention sur les constantes. Dans les paragraphes 4, 7, 8, et 9, on appellera constante un nombre réel ne dépendant que de $G, \phi, v_{0}$, et de la famille de normes $\left(\|\cdot\|_{v}\right)_{v \in \Sigma_{K}}$. Dans ce texte, une constante est donc en particulier indépendante du degré du corps $K$, de $V$, de $\mathbf{p}$ et de $u$. 


\section{4. Énoncés des théorèmes}

Dans toute la suite, on note $D=[K: \mathbb{Q}] /\left[K_{v_{0}}: \mathbb{Q}_{v_{0}}\right]$. Pour tout $i \in\{1, \ldots, n\}$, on pose $\rho_{i}=1$ si $G_{i}$ est linéaire, et $\rho_{i}=2$ sinon. Nous allons obtenir des minorations légèrement différentes selon que le groupe $G$ est une variété semi-abélienne ou non.

4.1. Cas général. Dans le cas où la place $v_{0}$ est archimédienne, nous obtenons le résultat général suivant.

THÉORÈmE 4.1. Il existe une constante $q_{3} \geq 1$ ayant la propriété suivante. Supposons que la place $v_{0}$ est archimédienne. Soit $\mathfrak{e}$ un nombre réel supérieur à e et soit $\mathfrak{a} \geq 1$ un nombre réel tel que

$$
\mathfrak{a} \geq \frac{\max \left\{D, D h(V), \log \|u\|_{v_{0}}\right\}}{\log \mathfrak{e}} .
$$

Posons

$$
U_{0}:=(\mathfrak{a} \log \mathfrak{e}) \mathfrak{a}^{1 / t} \times \prod_{i=1}^{n}\left(1+\frac{D \max _{k \leq \mathfrak{a}} h\left(k \mathbf{p}_{i}\right)+\left(\mathfrak{a} \mathfrak{e}\left\|u_{i}\right\|_{v_{0}}\right)^{\rho_{i}}}{\mathfrak{a} \log \mathfrak{e}}\right)^{g_{i} / t},
$$

où le maximum porte sur les entiers naturels $k \leq \mathfrak{a}$. Supposons que pour tout sous-groupe algébrique connexe $G^{\prime}$ de $G$ tel que $t_{G^{\prime}}+V \otimes_{K} \overline{\mathbb{Q}} \neq t_{G}$, on ait $u \notin t_{G^{\prime}}\left(\mathbb{C}_{v_{0}}\right)$. Alors $u \notin V \otimes_{K} \mathbb{C}_{v_{0}}$ et

$$
\log d(u, V) \geq-q_{3} U_{0} .
$$

L'affirmation $u \notin V \otimes_{K} \mathbb{C}_{v_{0}}$ du théorème est une conséquence triviale de l'hypothèse faite sur $u$, et l'intérêt du théorème réside dans la minoration de la distance $d(u, V)$. Remarquons que notre résultat améliore la minoration du théorème 1.2 de [21], dans laquelle le terme $\mathfrak{a}^{1 / t}$ était remplacé par

$$
\left(\mathfrak{a}+\frac{D}{\log \mathfrak{e}} \log \left(e+\frac{D}{\log \mathfrak{e}}\right)\right)^{1 / t} .
$$

Nous remplaçons également les quantités $\max _{k \leq \mathfrak{G} \mathbf{q}^{\mathfrak{a}}} h\left(k \mathbf{p}_{i}\right)$ de 21] par $\max _{k \leq \mathfrak{a}} h\left(k \mathbf{p}_{i}\right)$. Mais comme nous l'avons annoncé en introduction, l'intérêt principal du théorème 4.1 est de supprimer l'hypothèse faite sur le point $\mathbf{p}$ dans [21] (qui rendait par exemple le théorème 1.2 de [21] inapplicable si $\mathbf{p}$ était un point de torsion). L'hypothèse $t_{G^{\prime}}+V \otimes \overline{\mathbb{Q}}$ $\neq t_{G} \Rightarrow u \notin t_{G^{\prime}}\left(\mathbb{C}_{v_{0}}\right)$ qui subsiste dans le théorème 4.1 est courante dans les résultats de minorations simultanées de formes linéaires de logarithmes [29, 34. Quand $V$ est un hyperplan $(t=1)$, cette hypothèse correspond simplement à la condition naturelle $u \notin V \otimes \mathbb{C}_{v_{0}}$.

Nous démontrons également un analogue ultramétrique de ce théorème, qui généralise le théorème 1.3 de [21]. Rappelons que pour tout nombre réel $a$, on a noté $\log _{+}(a)=$ $\log \max \{1, a\}$.

THÉORÈME 4.2. Il existe une constante $q_{4} \geq 1$ ayant la propriété suivante. Supposons que la place $v_{0}$ est finie et que $\|u\|_{v_{0}}<r_{p_{0}}$. Soit $\mathfrak{R}$ un nombre réel dans l'intervalle ] $1, r_{p_{0}} /\|u\|_{v_{0}}[$ et soit $\mathfrak{a} \geq 1$ un nombre réel supérieur à

$$
\frac{D \max \{1, h(V)\}+\log _{+}\left((\log \mathfrak{R})^{-1}\right)}{\log \mathfrak{R}} .
$$


Posons

$$
U_{1}:=(\mathfrak{a} \log \mathfrak{R}) \mathfrak{a}^{1 / t} \prod_{i=1}^{n}\left(1+\frac{D \max _{k \leq \mathfrak{a}} h\left(k \mathbf{p}_{i}\right)}{\mathfrak{a} \log \mathfrak{R}}\right)^{g_{i} / t},
$$

où le maximum porte sur les entiers naturels $k \leq \mathfrak{a}$. Supposons que pour tout sous-groupe algébrique connexe $G^{\prime}$ de $G$ tel que $t_{G^{\prime}}+V \otimes \overline{\mathbb{Q}} \neq t_{G}$, on ait $u \notin t_{G^{\prime}}\left(\mathbb{C}_{v_{0}}\right)$. Alors on a $u \notin V \otimes_{K} \mathbb{C}_{v_{0}}$ et

$$
\log d(u, V) \geq-q_{4} U_{1} .
$$

Dans ce théorème intervient l'hypothèse naturelle que le point $u$ appartient au disque de convergence de l'exponentielle. Ceci améliore le théorème 1.3 de [21, qui imposait la condition plus forte $\|u\|_{v_{0}}<r_{p_{0}}^{2}$. Cette nouveauté est due à l'utilisation d'un cas très particulier d'un lemme d'interpolation dû à Robba [36] (rappelé au paragraphe 6). Comme dans le cas archimédien, nous remplaçons les quantités

$$
\left(\mathfrak{a}+\frac{D}{\log \mathfrak{e}} \log \left(e+\frac{D}{\log \mathfrak{e}}\right)\right)^{1 / t} \text { et } \max _{k \leq \Psi^{\mathfrak{a}}} h\left(k \mathbf{p}_{i}\right)
$$

du théorème 1.3 de [21] par $\mathfrak{a}^{1 / t}$ et $\max _{k \leq \mathfrak{a}} h\left(k \mathbf{p}_{i}\right)$ respectivement. Remarquons que dans le cas ultramétrique, l'hypothèse $t_{G^{\prime}}+V \otimes \overline{\mathbb{Q}} \neq t_{G} \Rightarrow u \notin t_{G^{\prime}}\left(\mathbb{C}_{v_{0}}\right)$ est équivalente à celle du théorème 1.3 de [21, car l'exponentielle $v_{0}$-adique réalise un difféomorphisme de $D\left(0, r_{p_{0}}\right)$ sur son image. Ainsi, si $k$ est un entier non nul et s'il existe un sous-groupe $G^{\prime}$ tel que $k \mathbf{p} \in G^{\prime}(\overline{\mathbb{Q}})$, alors on a $u \in t_{G^{\prime}}\left(\mathbb{C}_{v_{0}}\right)$.

4.2. Cas semi-abélien. Dans le cas où le groupe $G$ est une variété semi-abélienne, nous obtenons les deux énoncés suivants, qui sont plus précis en terme de la hauteur du sous-espace $V$. Nous établissons les minorations de [21, théorèmes 1.2 et 1.3] avec des hypothèses plus faibles sur le vecteur $u$.

THÉORÈme 4.3. Supposons que le groupe G est semi-abélien et que la place $v_{0}$ est archimédienne. Il existe une constante $q_{5} \geq 1$ ayant la propriété suivante. Soit $\mathfrak{e}$ un nombre réel supérieur à e et soit $\mathfrak{a} \geq 1$ un nombre réel tel que

$$
\mathfrak{a} \geq \frac{\max \left\{D, D h(V), \log \|u\|_{v_{0}}\right\}}{\log \mathfrak{e}} .
$$

Posons

$$
\begin{aligned}
U_{2}:= & (\mathfrak{a} \log \mathfrak{e})\left(1+\frac{D}{\log \mathfrak{e}} \log \left(\frac{D}{\log \mathfrak{e}}\right)\right)^{1 / t} \\
& \times \prod_{i=1}^{n}\left(1+\frac{D \max _{k \leq \mathfrak{a}} h\left(k \mathbf{p}_{i}\right)+\left(\mathfrak{a} \mathfrak{e}\left\|u_{i}\right\|_{v_{0}}\right)^{\rho_{i}}}{\mathfrak{a} \log \mathfrak{e}}\right)^{g_{i} / t},
\end{aligned}
$$

où le maximum porte sur les entiers naturels $k \leq \mathfrak{a}$. Supposons que pour tout sousgroupe algébrique connexe $G^{\prime}$ de $G$ tel que $t_{G^{\prime}}+V \otimes \overline{\mathbb{Q}} \neq t_{G}$, on ait $u \notin t_{G^{\prime}}\left(\mathbb{C}_{v_{0}}\right)$. Alors $u \notin V \otimes_{K} \mathbb{C}_{v_{0}}$ et

$$
\log d(u, V) \geq-q_{5} U_{2} .
$$

THÉORÈme 4.4. Supposons que le groupe $G$ est semi-abélien, que la place $v_{0}$ est finie et que $\|u\|_{v_{0}}<r_{p_{0}}$. Il existe une constante $q_{6} \geq 1$ ayant la propriété suivante. Soit $\mathfrak{R}$ un 
nombre réel dans l'intervalle $] 1, r_{p_{0}} /\|u\|_{v_{0}}[$ et soit $\mathfrak{a} \geq 1$ un nombre réel supérieur à

$$
\frac{D \max \{1, h(V)\}+\log _{+}\left((\log \mathfrak{R})^{-1}\right)}{\log \mathfrak{R}} .
$$

Posons

$$
U_{3}:=(\mathfrak{a} \log \mathfrak{R})\left(1+\frac{D}{\log \mathfrak{R}} \log \left(\frac{D}{\log \mathfrak{R}}\right)\right)^{1 / t} \prod_{i=1}^{n}\left(1+\frac{D \max _{k \leq \mathfrak{a}} h\left(k \mathbf{p}_{i}\right)}{\mathfrak{a} \log \mathfrak{R}}\right)^{g_{i} / t},
$$

où le maximum porte sur les entiers naturels $k \leq \mathfrak{a}$. Supposons que pour tout sousgroupe algébrique connexe $G^{\prime}$ de $G$ tel que $t_{G^{\prime}}+V \otimes \overline{\mathbb{Q}} \neq t_{G}$, on ait $u \notin t_{G^{\prime}}\left(\mathbb{C}_{v_{0}}\right)$. Alors $u \notin V \otimes_{K} \mathbb{C}_{v_{0}}$ et

$$
\log d(u, V) \geq-q_{6} U_{3}
$$

4.3. Commentaires sur la démonstration. Le schéma de démonstration que nous proposons est commun aux quatre théorèmes. Comme annoncé dans l'introduction, il combine les outils classiques issus de la méthode de Baker, approfondie par Philippon et Waldschmidt [34, 35], avec des outils de la théorie des pentes des fibrés adéliques hermitiens. En particulier, la section auxiliaire sera construite au moyen du « lemme de Siegel approché absolu »(lemme 2.14), à la manière de Gaudron [24]. Au lieu de considérer un coefficient de Taylor de cette section et d'appliquer la formule du produit, nous encadrerons la hauteur de l'un de ses jets, ce qui nous affranchira de la dépendance en un choix de base de dérivation. Comme dans l'article [21, les estimations aux places ultramétriques reposent sur la notion de taille de sous-schémas formels due à Bost [8]. L'énoncé clef (théorème 5.1 qui nous permettra « d'exclure » le cas périodique en évitant le recours à une extrapolation sur les dérivations fait l'objet du paragraphe 5 . Nous l'utiliserons lors de la construction d'un sous-groupe obstructeur (lemme 8.3), ainsi que dans l'application d'un lemme de multiplicités (8.3. Le paragraphe 6 regroupe les lemmes d'interpolations que nous appliquerons au 8.8 . Nous aurons alors tous les résultats intermédiaires nécessaires pour démontrer les théorèmes que nous venons d'énoncer.

Nous commencerons par les démonstrations des théorèmes 4.3 et 4.4 , qui concernent le cas semi-abélien ( $\$ 8$. Nous appliquerons la méthode décrite ci-dessus en attachant un facteur $\mathbb{G}_{\mathrm{a}}$ au groupe $G$ : nous travaillerons avec le point $(1, \mathbf{p}) \in\left(\mathbb{G}_{\mathrm{a}} \times G\right)(K)$, et nous établirons une minoration de la distance $d\left((1, u), t_{\mathbb{G}_{\mathrm{a}}} \oplus V\right)=d(u, V)$. Nous disposerons ainsi d'une variable supplémentaire pour construire la section auxiliaire. L'influence du facteur $\mathbb{G}_{\mathrm{a}}$ se manifeste notamment lors de l'application du lemme de multiplicités (\$8.3), où il permet d'assouplir les contraintes que doivent vérifier les paramètres. Cet artifice nous permettra de préciser la mesure en termes du paramètre $\mathfrak{a}$, et donc en termes de la hauteur $h(V)$. En contrepartie, l'ajout du facteur $\mathbb{G}_{\mathrm{a}}$ introduit un terme résiduel, appelé «poids de la droite affine » (en suivant la terminologie de Gaudron [21]), qui sera évalué au paragraphe 8.10 . C'est ce terme qui fait apparaître les quantités

$$
\left(1+\frac{D}{\log \mathfrak{e}} \log \left(e+\frac{D}{\log \mathfrak{e}}\right)\right)^{1 / t} \text { et }\left(1+\frac{D}{\log \mathfrak{R}} \log \left(e+\frac{D}{\log \mathfrak{R}}\right)\right)^{1 / t}
$$

dans les minorations des théorèmes 4.3 et 4.4 . 
Dans un second temps, nous démontrerons les théorèmes 4.1 et 4.2 (\$9), où $G$ n'est plus nécessairement semi-abélien. La preuve est l'exact analogue de celle du $\$ 8$, sans l'ajout d'un facteur $\mathbb{G}_{\mathrm{a}}$. Dans le cas général, l'astuce précédente se révèle inutile dans la démonstration, car l'on ne sait pas décrire simplement les sous-groupes algébriques de $\mathbb{G}_{\mathrm{a}} \times \overline{\mathbb{Q}} G$. Ainsi, le poids de la droite affine n'intervient pas, et c'est ce qui permet de remplacer le terme $\left(\mathfrak{a}+\frac{D}{\log \mathfrak{e}} \log \left(e+\frac{D}{\log \mathfrak{e}}\right)\right)^{1 / t}$ de [21] par $\mathfrak{a}^{1 / t}$. Si nous avions proposé une démonstration commune aux quatre théorèmes, cette amélioration aurait été impossible; par ailleurs, nous aurions été contraint de faire une l'hypothèse plus forte sur le point $u$ dans le cas général, à savoir : pour tout sous-groupe algébrique connexe $G^{\prime}$ de $\mathbb{G}_{\mathrm{a}} \times \overline{\mathbb{Q}} G$ tel que $t_{G^{\prime}}+\left(t_{\mathbb{G}_{\mathrm{a}}} \oplus V \otimes_{K} \overline{\mathbb{Q}}\right) \neq t_{\mathbb{G}_{\mathrm{a}} \times G}$, on a $(1, u) \notin t_{\mathbb{G}_{\mathrm{a}} \times G}\left(\mathbb{C}_{v_{0}}\right)$. Afin de limiter les répétitions, nous renverrons régulièrement aux démonstrations du cas semi-abélien dans la preuve du cas général. Nous porterons néanmoins une attention particulière aux étapes qui requièrent des modifications (choix d'un sous-groupe, lemme de multiplicités et choix des paramètres).

\section{5. Énoncé clef}

Dans tout ce paragraphe, on fixe un groupe algébrique connexe et commutatif $\mathcal{G}$ sur $\overline{\mathbb{Q}}$. On considère un plongement $\phi$ de $\mathcal{G}$ dans un espace projectif $\mathbb{P} \frac{N}{\mathbb{Q}}$ et on note $\operatorname{deg} \mathcal{A}$ le degré relatif à $\phi$ d'un sous-groupe algébrique $\mathcal{A}$ de $\mathcal{G}$. Soit $K$ un corps de nombres sur lequel $\mathcal{G}$ est défini. On fixe une structure de fibré adélique hermitien sur $t_{\mathcal{G}}$ (vu comme un $K$-espace vectoriel). Si $\mathcal{G}^{\prime}$ est un sous-groupe de $\mathcal{G}$ défini sur $K$, on note $h\left(t_{\mathcal{G}^{\prime}}\right)$ la hauteur de $t_{\mathcal{G}^{\prime}}$ relativement à ce choix de structure adélique. Considérons une place archimédienne $v$ de $K$ et fixons une extension de cette place à $\overline{\mathbb{Q}}$. Pour tout sous-groupe algébrique $\mathcal{G}^{\prime}$ de $\mathcal{G}$ (défini sur $\overline{\mathbb{Q}}$ ), on note $t_{\mathcal{G}^{\prime}}\left(\mathbb{C}_{v}\right)=t_{\mathcal{G}^{\prime}} \otimes_{\overline{\mathbb{Q}}} \mathbb{C}_{v}$. Soit $\mathrm{d}_{v}$ une distance associée à une norme hermitienne donnée $\operatorname{sur} t_{\mathcal{G}}\left(\mathbb{C}_{v}\right)$. Notons exp: $t_{\mathcal{G}}\left(\mathbb{C}_{v}\right) \rightarrow \mathcal{G}\left(\mathbb{C}_{v}\right)$ l'exponentielle de $\mathcal{G}\left(\mathbb{C}_{v}\right)$ et $\Omega_{\mathcal{G}}$ le noyau de l'application exp (appelé réseau des périodes).

Le théorème suivant est l'ingrédient clef qui nous permettra d'exclure le cas périodique lors de la démonstration des théorèmes du paragraphe 4. Le premier point correspond au théorème 1.2 de l'introduction, et sera utilisé lors de la construction d'un sous-groupe obstructeur (lemmes 8.3 et 9.3). Le second point interviendra lors de l'utilisation d'un lemme de multiplicités, aux paragraphes 8.3 et 9.3 .

THÉORÈmE 5.1. Il existe une constante $q_{7} \geq 1$ vérifiant la propriété suivante. Soit $\mathcal{H} \subsetneq \mathcal{G}$ un sous-groupe algébrique connexe défini sur $K$ et soit $\mathrm{u} \in t_{\mathcal{G}}\left(\mathbb{C}_{v}\right)$ un vecteur tel que le point $\mathrm{p}:=\exp (\mathrm{u})$ appartienne à $\mathcal{G}(\overline{\mathbb{Q}})$. Soit $S$ un entier naturel non nul.

(1) Supposons que pour tout sous-groupe algébrique connexe $\mathcal{G}^{\prime}$ de $\mathcal{G}$ tel que $t_{\mathcal{G}^{\prime}}+t_{\mathcal{H}} \neq t_{\mathcal{G}}$, on ait $\mathrm{u} \notin t_{\mathcal{G}^{\prime}}\left(\mathbb{C}_{v}\right)$. Soit $\mathcal{G}^{\prime}$ un sous-groupe algébrique connexe de $\mathcal{G}$ tel que $t_{\mathcal{G}^{\prime}}+t_{\mathcal{H}}$ $\neq t_{\mathcal{G}} . S i$

$$
\mathrm{d}_{v}\left(\mathrm{u}, t_{\mathcal{H}}\left(\mathbb{C}_{v}\right)\right)<\frac{1}{S \operatorname{deg} \mathcal{G}^{\prime} \exp \left(q_{7} \max \left\{1, h\left(t_{\mathcal{H}}\right)\right\}\right)},
$$

alors

$$
\forall s \in\{1, \ldots, S\}, \quad s \mathrm{p} \notin \mathcal{G}^{\prime}(\overline{\mathbb{Q}}) .
$$


(2) Pour tout sous-groupe algébrique connexe $\mathcal{G}^{\prime}$ de $\mathcal{G}$ tel que $t_{\mathcal{H}} \subseteq t_{\mathcal{G}^{\prime}}$ et $\mathrm{u} \notin t_{\mathcal{G}^{\prime}}\left(\mathbb{C}_{v}\right)$, on a l'implication

$$
\mathrm{d}_{v}\left(\mathrm{u}, t_{\mathcal{H}}\left(\mathbb{C}_{v}\right)\right)<\frac{1}{q_{7} S \operatorname{deg} \mathcal{G}^{\prime}} \Longrightarrow \forall s \in\{1, \ldots, S\}, s \mathrm{p} \notin \mathcal{G}^{\prime}(\overline{\mathbb{Q}}) .
$$

5.1. Énoncé complet et idée de la démonstration. Au cours de la démonstration du théorème 5.1, nous établirons plusieurs résultats intermédiaires d'intérêt indépendant. Nous les regroupons dans l'énoncé ci-dessous, dont le théorème 5.1 est une conséquence immédiate.

THÉORÈmE 5.2. Il existe une constante $q \geq 1$ vérifiant la propriété suivante. Soit $\mathcal{H} \subsetneq \mathcal{G}$ un sous-groupe algébrique connexe et soit $\mathrm{u} \in t_{\mathcal{G}}\left(\mathbb{C}_{v}\right)$ un vecteur tel que le point $\mathrm{p}:=$ $\exp (\mathrm{u})$ appartienne à $\mathcal{G}(\overline{\mathbb{Q}})$. Soit $S$ un entier naturel non nul.

(1) Supposons que pour tout sous-groupe algébrique connexe $\mathcal{G}^{\prime}$ de $\mathcal{G}$ tel que $t_{\mathcal{G}^{\prime}}+t_{\mathcal{H}} \neq t_{\mathcal{G}}$, on ait $\mathrm{u} \notin t_{\mathcal{G}^{\prime}}\left(\mathbb{C}_{v}\right)$. Alors pour tout sous-groupe algébrique connexe $\mathcal{G}^{\prime}$ de $\mathcal{G}$ tel que $t_{\mathcal{G}^{\prime}}+t_{\mathcal{H}} \neq t_{\mathcal{G}}$, on a l'implication

$$
\mathrm{d}_{v}\left(\mathrm{u}, t_{\mathcal{H}}\left(\mathbb{C}_{v}\right)\right)<\frac{1}{{ }_{\mathbb{8}} S \operatorname{deg} \mathcal{G}^{\prime} \operatorname{deg} \mathcal{H}} \Longrightarrow \forall s \in\{1, \ldots, S\}, s \mathrm{p} \notin \mathcal{G}^{\prime}(\overline{\mathbb{Q}}) .
$$

(2) Pour tout sous-groupe algébrique connexe $\mathcal{G}^{\prime}$ tel que $t_{\mathcal{H}} \subseteq t_{\mathcal{G}^{\prime}}$ et $\mathrm{u} \notin t_{\mathcal{G}^{\prime}}\left(\mathbb{C}_{v}\right)$, on a

$$
\mathrm{d}_{v}\left(\mathrm{u}, t_{\mathcal{H}}\left(\mathbb{C}_{v}\right)\right)<\frac{1}{q_{\nabla} S \operatorname{deg} \mathcal{G}^{\prime}} \Longrightarrow \forall s \in\{1, \ldots, S\}, s \mathrm{p} \notin \mathcal{G}^{\prime}(\overline{\mathbb{Q}}) .
$$

(3) Supposons que $\mathrm{u} \notin t_{\mathcal{H}}\left(\mathbb{C}_{v}\right)$. Si

$$
\mathrm{d}_{v}\left(\mathrm{u}, t_{\mathcal{H}}\left(\mathbb{C}_{v}\right)\right)<\frac{1}{{ }_{8} S \operatorname{deg} \mathcal{H}},
$$

alors pour tout sous-groupe algébrique connexe $\mathcal{G}^{\prime}$ de $\mathcal{G}$ tel que $t_{\mathcal{G}^{\prime}} \subseteq t_{\mathcal{H}}$, on a

$$
\forall s \in\{1, \ldots, S\}, \quad s \mathrm{p} \notin \mathcal{G}^{\prime}(\overline{\mathbb{Q}}) .
$$

(4) Il existe une constante $\varphi_{9} \geq 1$ vérifiant la propriété suivante. Si $\mathcal{H}$ est défini sur $K$, les points $(1)$ et $(3)$ restent vrais en remplaçant le terme q $\mathrm{deg} \mathcal{H}$ par

$$
\exp \left(\varphi_{9} \max \left\{1, h\left(t_{\mathcal{H}}\right)\right\}\right) .
$$

Si $\mathcal{G}^{\prime}$ est défini sur $K$, alors le second point reste vrai en remplaçant q8 $\mathrm{deg} \mathcal{G}^{\prime}$ par

$$
\exp \left(q_{9} \max \left\{1, h\left(t_{\mathcal{G}^{\prime}}\right)\right\}\right) .
$$

Les trois premiers points de ce théorème permettent de comparer la distance entre un vecteur $\mathrm{u} \in t_{\mathcal{G}}\left(\mathbb{C}_{v}\right)$ et l'espace tangent $t_{\mathcal{G}^{\prime}}$ d'un sous-groupe algébrique $\mathcal{G}^{\prime}$ avec son degré $\operatorname{deg} \mathcal{G}^{\prime}$. En cela, on peut considérer ces énoncés comme des variantes du théorème suivant, dû à Bertrand et Philippon [3].

THÉORÈme 5.3 ([3] corollaire 2]). Il existe une constante q10 $>0$ telle que pour tout sous-groupe algébrique $\mathcal{A}$ de $\mathcal{G}$ et pour toute période $\omega \in \Omega_{\mathcal{G}} \backslash t_{\mathcal{A}}\left(\mathbb{C}_{v}\right)$, on a

$$
\mathrm{d}_{v}\left(\omega, t_{\mathcal{A}}\left(\mathbb{C}_{v}\right)\right) \geq \frac{1}{[10]} \operatorname{deg} \mathcal{A} .
$$


La démonstration du théorème 5.2 s'appuie en partie sur le lemme suivant. Il peut s'interpréter comme une légère généralisation du théorème 5.3 . valable pour un vecteur de $t_{\mathcal{G}}\left(\mathbb{C}_{v}\right)$ qui n'est pas nécessairement une période.

LEMme 5.4. Soit $S$ un entier naturel non nul et soit $\mathrm{u} \in t_{\mathcal{G}}\left(\mathbb{C}_{v}\right)$ un vecteur tel que le point $\mathrm{p}:=\exp (\mathrm{u})$ appartienne à $\mathcal{G}(\overline{\mathbb{Q}})$. Alors pour tout sous-groupe algébrique $\mathcal{A}$ de $\mathcal{G}$ tel que $\mathrm{u} \notin t_{\mathcal{A}}\left(\mathbb{C}_{v}\right)$, on a

$$
\mathrm{d}_{v}\left(\mathrm{u}, t_{\mathcal{A}}\left(\mathbb{C}_{v}\right)\right)<\frac{1}{\bar{q}_{10} S \operatorname{deg} \mathcal{A}} \Longrightarrow \forall s \in\{1, \ldots, S\}, s \mathrm{p} \notin \mathcal{A}(\overline{\mathbb{Q}}) .
$$

Démonstration. Raisonnons par contraposée et supposons qu'il existe un entier $s \in$ $\{1, \ldots, S\}$ tel que $s p \in \mathcal{A}(\overline{\mathbb{Q}})$. Alors il existe un vecteur $x \in t_{\mathcal{A}}\left(\mathbb{C}_{v}\right)$ et une période $\omega \in \Omega_{\mathcal{G}}$ tels que $s \mathrm{u}=x+\omega$. On a donc

$$
\mathrm{d}_{v}\left(\mathrm{u}, t_{\mathcal{A}}\left(\mathbb{C}_{v}\right)\right) \geq \frac{\mathrm{d}_{v}\left(s \mathrm{u}, t_{\mathcal{A}}\left(\mathbb{C}_{v}\right)\right)}{S}=\frac{\mathrm{d}_{v}\left(\omega, t_{\mathcal{A}}\left(\mathbb{C}_{v}\right)\right)}{S}
$$

Puisque $\mathrm{u} \notin t_{\mathcal{A}}\left(\mathbb{C}_{v}\right)$ et $x \in t_{\mathcal{A}}\left(\mathbb{C}_{v}\right)$, on a $\omega \notin t_{\mathcal{A}}\left(\mathbb{C}_{v}\right)$. D'après le théorème 5.3 .

ce qui démontre le lemme.

$$
\mathrm{d}_{v}\left(\mathrm{u}, t_{\mathcal{A}}\left(\mathbb{C}_{v}\right)\right) \geq \frac{1}{q_{10} S \operatorname{deg} \mathcal{A}}
$$

Les points (2) et (3) du théorème 5.2 se déduisent facilement du lemme 5.4. En effet, le deuxième point est une conséquence immédiate du lemme 5.4 (appliqué à $\mathcal{A}=\mathcal{G}^{\prime}$ ) une fois que l'on a remarqué que pour tout sous-groupe algébrique connexe $\mathcal{G}^{\prime}$ tel que $t_{\mathcal{H}} \subseteq t_{\mathcal{G}^{\prime}}$, on $\operatorname{a~}_{v}\left(\mathrm{u}, t_{\mathcal{G}^{\prime}}\left(\mathbb{C}_{v}\right)\right) \leq \mathrm{d}_{v}\left(\mathrm{u}, t_{\mathcal{H}}\left(\mathbb{C}_{v}\right)\right)$. Pour le troisième point, il suffit de remarquer que si $t_{\mathcal{G}^{\prime}} \subseteq t_{\mathcal{H}}$, alors $\mathcal{G}^{\prime}(\overline{\mathbb{Q}}) \subset \mathcal{H}(\overline{\mathbb{Q}})$ et d'appliquer le lemme 5.4 . Le reste du paragraphe 5 est consacré à la démonstration des points (1) et (4) du théorème 5.2. Le premier repose sur des propriétés des groupes algébriques engendrés par deux sous-groupes de $\mathcal{G}$, que nous établirons aux $\$ 5.2$. Nous en déduirons le point (4) grâce à des comparaisons entre le degré d'un sous-groupe algébrique et la hauteur de son espace tangent, démontrées au $\$ 5.3$. Nous terminerons par la preuve du théorème 5.2 au $\$ 5.4$.

5.2. Groupe engendré par des sous-groupes algébriques. On note $m: \mathcal{G} \times \overline{\mathbb{Q}} \mathcal{G} \rightarrow \mathcal{G}$ la loi de composition interne de $\mathcal{G}$. Si $S$ est un schéma sur Spec $\overline{\mathbb{Q}}$ et si $\mathbf{p}$ et $\mathbf{q}$ sont deux points de $\mathcal{G}(S)$, on notera pour simplifier $m(\mathbf{p}, \mathbf{q})=\mathbf{p}+\mathbf{q}$. Soient $\mathcal{A}$ et $\mathcal{B}$ deux sousgroupes algébriques de $\mathcal{G}$. Alors $\mathcal{A} \times_{\overline{\mathbb{Q}}} \mathcal{B}$ est un sous-groupe algébrique de $\mathcal{G} \times_{\overline{\mathbb{Q}}} \mathcal{G}$ et

$$
f=m_{\mid \mathcal{A} \times \overline{\mathbb{Q}}} \mathcal{B}: \mathcal{A} \times \overline{\mathbb{Q}} \mathcal{B} \rightarrow \mathcal{G}
$$

est un morphisme de groupes algébriques. On note $\mathcal{A}+\mathcal{B}=f\left(\mathcal{A} \times_{\overline{\mathbb{Q}}} \mathcal{B}\right)$. Pour tout Spec $\overline{\mathbb{Q}}$-schéma $S$,

$$
(\mathcal{A}+\mathcal{B})(S)=\{a+b \mid a \in \mathcal{A}(S), b \in \mathcal{B}(S)\}
$$

est un sous-groupe de $\mathcal{G}(S)$ par commutativité. De plus $\mathcal{A}+\mathcal{B}$ est fermé d'après 10 , proposition 2.7.1], donc c'est un sous-groupe algébrique de $\mathcal{G}$.

5.2.1. Décomposition de l'espace tangent. L'objectif de ce paragraphe est de démontrer le lemme suivant.

LEMmE 5.5. Les $\overline{\mathbb{Q}}$-espaces vectoriels $t_{\mathcal{A}+\mathcal{B}}$ et $t_{\mathcal{A}}+t_{\mathcal{B}}$ sont égaux. 
Démonstration. Comme $\mathcal{A}$ et $\mathcal{B}$ sont des sous-groupes algébriques de $\mathcal{A}+\mathcal{B}, t_{\mathcal{A}}+t_{\mathcal{B}}$ est un sous-espace vectoriel de $t_{\mathcal{A}+\mathcal{B}}$. Remarquons que $\mathcal{A} \cap \mathcal{B}$ est un sous-schéma en groupes fermé de $\mathcal{G}$. Pour tout schéma $S$ sur $\operatorname{Spec}(\overline{\mathbb{Q}})$, on a une suite exacte de groupes

$$
1 \rightarrow(\mathcal{A} \cap \mathcal{B})(S) \stackrel{\alpha}{\rightarrow}\left(\mathcal{A} \times_{\overline{\mathbb{Q}}} \mathcal{B}\right)(S) \stackrel{f}{\rightarrow}(\mathcal{A}+\mathcal{B})(S) \rightarrow 1,
$$

où $\alpha(g)=(g,-g)$ (rappelons que $f((a, b))=a+b)$. D'après [16, lemme 5.2.1(i)], on a donc une suite exacte

$$
0 \rightarrow t_{\mathcal{A} \cap \mathcal{B}} \rightarrow t_{\mathcal{A} \times \overline{\mathbb{Q}} \mathcal{B}} \rightarrow t_{\mathcal{A}+\mathcal{B}} \rightarrow 0 .
$$

De plus, on a $t_{\mathcal{A} \times \overline{\mathbb{Q}}}=t_{\mathcal{A}} \oplus t_{\mathcal{B}}$. D'après [38, II-V, §2.3)], les algèbres de Lie $t_{\mathcal{A} \cap \mathcal{B}} \otimes_{\overline{\mathbb{Q}}} \mathbb{C}$ et $\left(t_{\mathcal{A}} \cap t_{\mathcal{B}}\right) \otimes_{\overline{\mathbb{Q}}} \mathbb{C}$ sont égales, donc $\operatorname{dim}\left(t_{\mathcal{A} \cap \mathcal{B}}\right)=\operatorname{dim}\left(t_{\mathcal{A}} \cap t_{\mathcal{B}}\right)$. On en déduit que

$$
\operatorname{dim} t_{\mathcal{A}+\mathcal{B}}=\operatorname{dim} t_{\mathcal{A}}+\operatorname{dim} t_{\mathcal{B}}-\operatorname{dim}\left(t_{\mathcal{A}} \cap t_{\mathcal{B}}\right)=\operatorname{dim}\left(t_{\mathcal{A}}+t_{\mathcal{B}}\right),
$$

et donc $t_{\mathcal{A}}+t_{\mathcal{B}}=t_{\mathcal{A}+\mathcal{B}}$.

5.2.2. Majoration du degré d'un groupe engendré par des sous-groupes algébriques. On considère un recouvrement ouvert $\left(U_{i}\right)_{i \in I}$ de $\mathcal{G}$ et des $(N+1)$-uplets de polynômes $\left(P_{i}(\mathbf{X}, \mathbf{Y})=\left(P_{i, 0}(\mathbf{X}, \mathbf{Y}), \ldots, P_{i, N}(\mathbf{X}, \mathbf{Y})\right)_{i \in I}\right.$ de l'anneau $\overline{\mathbb{Q}}[\mathbf{X}, \mathbf{Y}]$, homogènes en chacune des variables $\mathbf{X}:=\left(X_{0}, \ldots, X_{N}\right)$ et $\mathbf{Y}:=\left(Y_{0}, \ldots, Y_{N}\right)$ et représentant localement la loi de composition interne de $\mathcal{G}$ : si p, q sont des points de $U_{i}(\overline{\mathbb{Q}})$, on a ainsi

$$
\phi(\mathbf{p}+\mathbf{q})=\left(P_{i, 0}(\phi(\mathbf{p}), \phi(\mathbf{q})): \cdots: P_{i, N}(\phi(\mathbf{p}), \phi(\mathbf{q}))\right) .
$$

Soit $q_{11} \geq 1$ un majorant des degrés de ces polynômes.

LEMME 5.6. L'inégalité suivante est vérifiée :

$$
\operatorname{deg}(\mathcal{A}+\mathcal{B}) \leq q \frac{\operatorname{dim}}{111} \mathcal{G}(2 \operatorname{dim} \mathcal{G}) ! \operatorname{deg} \mathcal{A} \operatorname{deg} \mathcal{B} .
$$

Démonstration. Rappelons que $f$ désigne la restriction de $m$ à $\mathcal{A} \times_{\overline{\mathbb{Q}}} \mathcal{B}$. On note $V_{i}:=$ $U_{i} \cap(\mathcal{A}+\mathcal{B})$ pour tout $i \in I$. Remarquons les fibres de $f$ sont toutes isomorphes (en tant que schémas sur Spec $\overline{\mathbb{Q}})$ car $f^{-1}(x)=m_{\mathcal{G} \times \mathcal{G}}\left(f^{-1}(y),\left(y^{-1}, x\right)\right)$ pour tout couple $(x, y)$ de points de $\mathcal{A}+\mathcal{B}$ (rappelons que le groupe $\mathcal{G}$ est commutatif). Le lemme 5.6 est alors une conséquence de [3, proposition 2] une fois que l'on a remarqué que $\operatorname{deg}(\mathcal{A} \times \overline{\mathbb{Q}} \mathcal{B}) \leq$ $(2 \operatorname{dim} \mathcal{G}) ! \operatorname{deg} \mathcal{A} \operatorname{deg} \mathcal{B}$. Pour le confort du lecteur, nous reprenons les arguments de la démonstration ici. On a l'égalité

$$
\operatorname{deg}(\mathcal{A}+\mathcal{B})=\operatorname{card}((\mathcal{A}+\mathcal{B}) \cap Y)
$$

où $Y$ est une sous-variété linéaire de $\mathbb{P} \frac{N}{\mathbb{Q}}$ en position générale de codimension $d=$ $\operatorname{dim}(\mathcal{A}+\mathcal{B})$. Considérons des formes linéaires $L_{1}, \ldots, L_{d}$ définissant $Y$ dans $\mathbb{P} \frac{N}{\bar{Q}}$. Notons $Y^{\prime}$ la sous-variété de $\mathbb{P}_{\overline{\mathbb{Q}}}^{N} \times_{\overline{\mathbb{Q}}} \mathbb{P}_{\overline{\mathbb{Q}}}^{N}$ définie par les polynômes homogènes $L_{j}\left(P_{i}(\mathbf{X}, \mathbf{Y})\right)$, $i \in I, 1 \leq j \leq d$. Ces polynômes sont de degré inférieur à q11, Posons $Z^{\prime}=Y^{\prime} \cap(\mathcal{A} \times \overline{\mathbb{Q}} \mathcal{B})$. D'après [32, proposition 3.3], on a l'inégalité

$$
\operatorname{deg} Z^{\prime} \leq q \frac{\operatorname{dim}}{[11} \mathcal{G} \operatorname{deg}(\mathcal{A} \times \overline{\mathbb{Q}} \mathcal{B}) \leq q \frac{\operatorname{dim}}{111} \mathcal{G}(2 \operatorname{dim} \mathcal{G}) ! \operatorname{deg} \mathcal{A} \operatorname{deg} \mathcal{B}
$$

Comme $Z^{\prime}$ est la réunion $\operatorname{de} \operatorname{deg}(\mathcal{A}+\mathcal{B})$ fibres de $f$ d'après l'égalité (5.1), on a par ailleurs

$$
\operatorname{deg} Z^{\prime} \geq \operatorname{deg}(\mathcal{A}+\mathcal{B}) \min _{x \in(\mathcal{A}+\mathcal{B}) \cap Y} \operatorname{deg} f^{-1}(x) \geq \operatorname{deg}(\mathcal{A}+\mathcal{B})
$$


(voir [39, proposition 8.3]). En comparant avec la majoration de deg $Z^{\prime}$ obtenue précédemment, on obtient le résultat voulu.

5.3. Degré d'un sous-groupe et hauteur de l'espace tangent. Étant donné un sous-groupe algébrique $\mathcal{G}^{\prime}$ de $\mathcal{G}$ défini sur $K$, l'objectif de ce paragraphe est de comparer le degré $\operatorname{deg}\left(\mathcal{G}^{\prime}\right)$ de $\mathcal{G}^{\prime}$ avec la hauteur $h\left(t_{\mathcal{G}^{\prime}}\right)$ de son espace tangent. Nous étudierons d'abord le cas où $\mathcal{G}$ est une variété abélienne ou un groupe algébrique linéaire, avant de passer au cas général par un « dévissage » au moyen d'un théorème de décomposition dû à Chevalley. Dans la suite, si $\mathcal{G}^{\prime}$ est groupe algébrique commutatif, on note $\left(\mathcal{G}^{\prime}\right)^{\circ}$ la composante neutre de $\mathcal{G}^{\prime}$ (c'est-à-dire la composante connexe contenant l'élément neutre). On a alors $t_{\mathcal{G}^{\prime}}=t_{\left(\mathcal{G}^{\prime}\right)^{\circ}}$.

5.3.1. Cas d'une variété abélienne. Soit $\mathcal{A}$ une variété abélienne définie sur $K$. Supposons donnée une structure de fibré adélique hermitien sur le $K$-espace vectoriel $t_{\mathcal{A}}$ et fixons un plongement projectif de $\mathcal{A}$. Pour toute sous-variété abélienne $\mathcal{B}$ de $\mathcal{A}$, on note $\operatorname{deg} \mathcal{B}$ le degré de $\mathcal{B}$ associé à ce plongement. Le résultat suivant est une conséquence de [20, lemme 4.8].

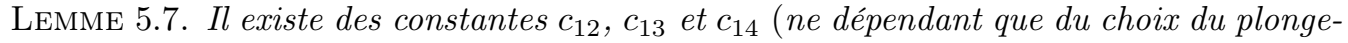
ment projectif de $\mathcal{A}$ et de la structure de fibré adélique hermitien de $\left.t_{\mathcal{A}}\right)$ telles que pour toute sous-variété abélienne $\mathcal{B}$ de $\mathcal{A}$, on ait

$$
q_{12} \log (\operatorname{deg} \mathcal{B})-q_{13} \leq h\left(t_{\mathcal{B}}\right) \leq q_{14} \log (\operatorname{deg} \mathcal{B})+q_{13} .
$$

Démonstration. Supposons que la structure de fibré adélique hermitien sur $t_{\mathcal{A}}$ est associée aux formes de Riemann d'un fibré en droites très ample $\mathscr{L}$ sur $\mathcal{A}$ comme dans [20, pages 702-703], et que le plongement projectif de $\mathcal{A}$ correspond au choix d'une base de sections globales de $\mathscr{L}$. D'après [20, lemme 4.8], pour toute sous-variété abélienne $\mathcal{B}$ de $\mathcal{A}$, on a dans ce cas

$$
\begin{aligned}
\log \left(\frac{\operatorname{deg} \mathcal{B}}{(\operatorname{dim} \mathcal{B}) !}\right)-3 \operatorname{dim} \mathcal{B} & \leq 2 h\left(t_{\mathcal{B}}\right. \\
& \leq 2 h_{F}(\mathcal{A})+8 \operatorname{dim} \mathcal{A} \log \left(\frac{\operatorname{deg} \mathcal{B}}{(\operatorname{dim} \mathcal{B}) !}\right)+2 \operatorname{codim}_{\mathcal{A}} \mathcal{B}
\end{aligned}
$$

où $h_{F}(\mathcal{A})$ désigne la hauteur de Faltings de $\mathcal{A}$, normalisée comme dans l'article [20]. Par ailleurs, un nouveau nouveau choix de structure de fibré adélique sur $t_{\mathcal{A}}$ ne modifie $h\left(t_{\mathcal{B}}\right)$ qu'à constante près. De même, un changement de plongement projectif de $\mathcal{A}$ ne modifie $\operatorname{deg} \mathcal{B}$ qu'à constante près (voir [3, remarque 2]). Le lemme est donc une conséquence de l'encadrement 5.2 .

5.3.2. Cas d'un groupe linéaire. Traitons maintenant le cas où le groupe algébrique est linéaire et commutatif. Nous allons d'abord nous intéresser au cas du tore $\mathcal{L}=\mathbb{G}_{\mathrm{m}}^{n}$, où $n \in \mathbb{N} \backslash\{0\}$. On suppose donnée une $K$-base $\left(e_{1}, \ldots, e_{n}\right)$ de $t_{\mathcal{L}}$, et on considère la structure de fibré vectoriel adélique hermitien associée (voir l'exemple 2.3 à la page 10). Si $\mathcal{L}^{\prime}$ un sous-groupe algébrique connexe de $\mathcal{L}$, le réseau des périodes $\Omega_{\mathcal{L}^{\prime}}$ de $\mathcal{L}^{\prime}$ est un sous groupe de $(2 i \pi \mathbb{Z})^{n} \subset \mathbb{C}^{n}$. On fixe une structure d'espace vectoriel euclidien sur $\Omega_{\mathcal{L}} \otimes_{\mathbb{Z}} \mathbb{R}$ et on note $\operatorname{vol}\left(\Omega_{\mathcal{L}^{\prime}}\right)$ le volume fondamental de $\Omega_{\mathcal{L}^{\prime}}$. 
LEMME 5.8. Il existe une constante $q_{15}$ telle que pour tout sous-groupe algébrique connexe $\mathcal{L}^{\prime}$ de $\mathcal{L}$ défini sur $K$, on ait

$$
\log \left(\operatorname{vol}\left(\Omega_{\mathcal{L}^{\prime}}\right)\right)-q_{15} \leq h\left(t_{\mathcal{L}^{\prime}}\right) \leq \log \left(\operatorname{vol}\left(\Omega_{\mathcal{L}^{\prime}}\right)\right)+q_{15} .
$$

Démonstration. Considérons un sous-réseau $\Lambda$ de $\mathbb{Z}^{n} \simeq \bigoplus_{i=1}^{n} \mathbb{Z} e_{i}$ vérifiant

$$
\mathcal{L}^{\prime}(K)=\left\{\left(x_{1}, \ldots, x_{n}\right) \in \mathbb{G}_{\mathrm{m}}^{n}(K) \mid \prod_{i=1}^{n} x_{i}^{\lambda_{i}}=1 \forall\left(\lambda_{1}, \ldots, \lambda_{n}\right) \in \Lambda\right\} .
$$

On a dans ce cas

$$
t_{\mathcal{L}^{\prime}}=\left\{\left(z_{1}, \ldots, z_{n}\right) \in K^{n} \mid \sum_{i=1}^{n} \lambda_{i} z_{i}=0 \forall\left(\lambda_{1}, \ldots, \lambda_{n}\right) \in \Lambda\right\} .
$$

Comme $\mathcal{L}^{\prime}$ est connexe, il est irréductible, donc $\Lambda$ est primitif d'après [42, corollaire 4.5]). On a l'égalité $t_{\mathcal{L}^{\prime}}=\Lambda^{\perp} \otimes_{\mathbb{Z}} K$, où $\Lambda^{\perp}$ est le sous-réseau de $\mathbb{Z}^{n}$ défini par

$$
\Lambda^{\perp}:=\left\{\left(z_{1}, \ldots, z_{n}\right) \in \mathbb{Z}^{n} \mid \sum_{i=1}^{n} \lambda_{i} z_{i}=0 \forall\left(\lambda_{1}, \ldots, \lambda_{n}\right) \in \Lambda\right\} .
$$

Considérons une base $\left(\omega_{1}, \ldots, \omega_{r}\right)$ du $\mathbb{Z}$-module libre $\Lambda^{\perp}$. C'est une base du $K$-espace vectoriel $t_{\mathcal{L}^{\prime}}=\Lambda^{\perp} \otimes_{\mathbb{Z}} K$. Par définition de la hauteur, on a

$$
\begin{aligned}
h\left(t_{\mathcal{L}^{\prime}}\right) & =\sum_{v \in \Sigma_{K}} \frac{\left[K_{v}: \mathbb{Q}_{v}\right]}{[K: \mathbb{Q}]} \log \left\|\omega_{1} \wedge \ldots \wedge \omega_{r}\right\|_{v} \\
& =\log \left\|\omega_{1} \wedge \cdots \wedge \omega_{r}\right\|_{\infty}+\sum_{p \text { premier }} \log \left\|\omega_{1} \wedge \cdots \wedge \omega_{r}\right\|_{p} .
\end{aligned}
$$

La seconde égalité est justifiée par le fait que les coordonnées des vecteurs $\omega_{1}, \ldots, \omega_{r}$ dans la base $\left(e_{1}, \ldots, e_{n}\right)$ appartiennent à $\mathbb{Z}$. Notons $A$ la matrice de $M_{n, r}(\mathbb{Z})$ dont les vecteurs colonnes sont les coordonnées des vecteurs $\omega_{1}, \ldots, \omega_{r}$ de $\mathbb{Z}^{n}$. Par la formule de Cauchy-Binet,

$$
\left\|\omega_{1} \wedge \cdots \wedge \omega_{r}\right\|_{\infty}=\left|\left(\operatorname{det} A_{0}\right)_{A_{0}}\right|_{\infty}=\left|\operatorname{det}^{t} A A\right|^{1 / 2},
$$

où $A_{0}$ parcourt les mineurs de taille $r \times r \operatorname{de} A$. Par ailleurs, comme $\operatorname{det} A_{0} \in \mathbb{Z}$ pour tout mineur $A_{0}$ de taille $r \times r$, on a

$$
\prod_{p \text { premier }}\left\|\omega_{1} \wedge \cdots \wedge \omega_{r}\right\|_{p}=\prod_{p \text { premier }} \max _{A_{0}}\left|\operatorname{det} A_{0}\right|_{p}=\prod_{p \text { premier }}\left|\operatorname{pgcd}\left(\operatorname{det} A_{0}\right)_{A_{0}}\right|_{p} .
$$

La formule du produit entraîne alors

$$
\exp h\left(t_{\mathcal{L}^{\prime}}\right)=\frac{\left|\operatorname{det}{ }^{t} A A\right|^{1 / 2}}{\left|\operatorname{pgcd}\left(\operatorname{det} A_{0}\right)_{A_{0}}\right|},
$$

où le pgcd est pris sur l'ensemble des mineurs de taille $r \times r$ de $A$. Comme $\mathcal{L}^{\prime}$ est connexe, ce pgcd est égal à 1 d'après [42, proposition 4.2]. Par ailleurs, on peut identifier le $\mathbb{Z}$ module $\Omega_{\mathcal{L}}$ à $\mathbb{Z}^{n} \simeq \bigoplus_{i=1}^{n} \mathbb{Z} e_{i}$ en associant à $e_{k}$ la période $2 i \pi$ du $k$-ième facteur de $\mathbb{G}_{\mathrm{m}}^{n}$. Si $\Omega_{\mathcal{L}} \otimes_{\mathbb{Z}} \mathbb{R}$ est muni de la structure euclidienne correspondante, alors

$$
\operatorname{vol}\left(\Omega_{\mathcal{L}^{\prime}}\right)=\operatorname{vol}\left(\Lambda^{\perp}\right)=\left|\operatorname{det}^{t} A A\right|^{1 / 2},
$$


et donc $h\left(t_{\mathcal{L}^{\prime}}\right)=\log \left(\operatorname{vol}\left(\Omega_{\mathcal{L}^{\prime}}\right)\right)$. D'après [3, lemme 1], un changement de structure euclidienne sur $\Omega_{\mathcal{L}} \otimes_{\mathbb{Z}} \mathbb{R}$ ne modifie $\operatorname{vol}\left(\Omega_{\mathcal{L}^{\prime}}\right)$ qu'à une constante près, indépendante de $\mathcal{L}^{\prime}$. Le lemme est ainsi démontré.

Lemme 5.9. Soit $\mathcal{L}$ un groupe algébrique linéaire, connexe et commutatif, défini sur $K$. On considère un plongement $\phi$ de $\mathcal{L}$ dans un espace projectif et on note $\operatorname{deg}\left(\mathcal{L}^{\prime}\right)$ le degré d'un sous-groupe algébrique $\mathcal{L}^{\prime}$ de $\mathcal{L}$ associé à ce plongement. Pour toute structure de fibré adélique hermitien sur $t_{\mathcal{L}}$, il existe une constante $q_{16}$ telle que pour tout sous-groupe algébrique linéaire $\mathcal{L}^{\prime}$ de $\mathcal{L}$ défini sur $K$, on ait

$$
\log \left(\operatorname{deg} \mathcal{L}^{\prime}\right) \leq h\left(t_{\mathcal{L}^{\prime}}\right)+q 16 .
$$

Si de plus $\mathcal{L}$ est un tore, on a également

$$
h\left(t_{\mathcal{L}^{\prime}}\right)-q_{16} \leq \log \left(\operatorname{deg} \mathcal{L}^{\prime}\right) .
$$

Démonstration. Il existe une extension finie $K^{\prime}$ de $K$ et des entiers naturels $\ell, n$ tels que $\mathcal{L}_{K^{\prime}}=\mathcal{L} \times_{K} \operatorname{Spec} K^{\prime}$ soit isomorphe à $\left(\mathbb{G}_{\mathrm{a}, K^{\prime}}\right)^{\ell} \times_{K^{\prime}}\left(\mathbb{G}_{\mathrm{m}, K^{\prime}}\right)^{n}$. La hauteur $h\left(t_{\mathcal{L}^{\prime}}\right)$ et le degré $\operatorname{deg}\left(\mathcal{L}^{\prime}\right)$ intervenant dans le lemme étant invariants par extension de corps, on peut supposer sans perte de généralité que $K^{\prime}=K$. Ainsi, il existe un sous-groupe $\mathcal{L}_{\text {a }}^{\prime}$ (respectivement $\left.\mathcal{L}_{\mathrm{m}}^{\prime}\right)$ de $\left(\mathbb{G}_{\mathrm{a}, K}\right)^{\ell}$ (respectivement de $\left.\left(\mathbb{G}_{\mathrm{m}, K}\right)^{n}\right)$ tels que $\mathcal{L}^{\prime}$ soit isomorphe à $\mathcal{L}_{\mathrm{a}}^{\prime} \times_{K} \mathcal{L}_{\mathrm{m}}^{\prime}$. D'après [3, proposition 5 et remarque 2], il existe une constante $c \neq 0$ ne dépendant pas de $\mathcal{L}^{\prime}$ telle que

$$
\frac{1}{c} \operatorname{deg} \mathcal{L}^{\prime} \leq\left[\mathcal{L}^{\prime}:\left(\mathcal{L}^{\prime}\right)^{\circ}\right] \operatorname{vol}\left(\Omega_{\mathcal{L}^{\prime}}\right) \leq c \operatorname{deg} \mathcal{L}^{\prime},
$$

où $\left[\mathcal{L}^{\prime}:\left(\mathcal{L}^{\prime}\right)^{\circ}\right]$ désigne le nombre de composantes connexes de $\mathcal{L}^{\prime}$. Comme

$$
\left[\mathcal{L}^{\prime}:\left(\mathcal{L}^{\prime}\right)^{\circ}\right]=\left[\mathcal{L}_{\mathrm{m}}^{\prime}:\left(\mathcal{L}_{\mathrm{m}}^{\prime}\right)^{\circ}\right] \text { et } \operatorname{vol}\left(\Omega_{\mathcal{L}^{\prime}}\right)=\operatorname{vol}\left(\Omega_{\mathcal{L}_{\mathrm{m}}^{\prime}}\right),
$$

on en déduit que

$$
\frac{1}{c} \operatorname{deg} \mathcal{L}^{\prime} \leq\left[\mathcal{L}_{\mathrm{m}}^{\prime}:\left(\mathcal{L}_{\mathrm{m}}^{\prime}\right)^{\circ}\right] \operatorname{vol}\left(\Omega_{\mathcal{L}_{\mathrm{m}}^{\prime}}\right)=\operatorname{vol}\left(\Omega_{\left(\mathcal{L}_{\mathrm{m}}^{\prime}\right)^{\circ}}\right) \leq c \operatorname{deg} \mathcal{L}^{\prime}
$$

D'après le lemme 5.8 , il existe une constante $c^{\prime} \neq 0$ telle que

$$
\frac{1}{c^{\prime}} \operatorname{vol}\left(\Omega_{\left(\mathcal{L}_{\mathrm{m}}^{\prime}\right)^{\circ}}\right) \leq \exp h\left(t_{\mathcal{L}_{\mathrm{m}}^{\prime}}\right) \leq c^{\prime} \operatorname{vol}\left(\Omega_{\left(\mathcal{L}_{\mathrm{m}}^{\prime}\right)^{\circ}}\right)
$$

$\left(\operatorname{car} h\left(t_{\mathcal{L}_{\mathrm{m}}^{\prime}}\right)=h\left(t_{\left(\mathcal{L}_{\mathrm{m}}^{\prime}\right)^{\circ}}\right)\right)$. Dans le cas où $\mathcal{L}$ est un tore, on a $\mathcal{L}^{\prime}=\mathcal{L}_{\mathrm{m}}^{\prime}$ et le lemme est démontré. Passons maintenant au cas général. Un changement de structure de fibré adélique hermitien sur $t_{\mathcal{L}}$ ne modifie la hauteur $h\left(t_{\mathcal{L}^{\prime}}\right)$ qu'à constante près. On peut donc se contenter de démontrer le lemme pour une structure particulière et supposer que $t_{\mathbb{G}_{a}^{\ell}}$ et $t_{\mathbb{G}_{\mathrm{m}}^{n}}$ sont orthogonaux et que $\widehat{\mu}_{\max }\left(t_{\mathbb{G}_{\mathrm{a}}^{\ell}}\right)=0$. On a ainsi

$$
h\left(t_{\mathcal{L}_{\mathrm{a}}^{\prime}}\right)=-\widehat{\operatorname{deg}}_{\mathrm{n}}\left(t_{\mathcal{L}_{\mathrm{a}}^{\prime}}\right) \geq 0 \quad \text { et } \quad h\left(t_{\mathcal{L}^{\prime}}\right)=h\left(t_{\mathcal{L}_{\mathrm{a}}^{\prime}}\right)+h\left(t_{\mathcal{L}_{\mathrm{m}}^{\prime}}\right) \geq h\left(t_{\mathcal{L}_{\mathrm{m}}^{\prime}}\right)
$$

Finalement,

$$
\operatorname{deg} \mathcal{L}^{\prime} \leq c c^{\prime} \exp h\left(t_{\mathcal{L}^{\prime}}\right)
$$

et le lemme est démontré. 
5.3.3. Cas général. Soit $\mathcal{L}$ le sous-groupe algébrique linéaire connexe maximal de $\mathcal{G}$. D'après le théorème de décomposition de Chevalley (voir [13, théorème 1.1]), on dispose d'une suite exacte de groupes algébriques

$$
1 \rightarrow \mathcal{L} \stackrel{\iota}{\rightarrow} \mathcal{G} \stackrel{\pi}{\rightarrow} \mathcal{A} \rightarrow 1,
$$

où $\mathcal{A}$ est une variété abélienne définie sur $K$. Nous allons démontrer la proposition suivante en appliquant les lemmes 5.7 et 5.9 .

Proposition 5.10. Il existe des constantes q17 et q18 telles que pour tout sous-groupe algébrique connexe $\mathcal{G}^{\prime}$ de $\mathcal{G}$ défini sur $K$, on ait

$$
\log \left(\operatorname{deg} \mathcal{G}^{\prime}\right) \leq q_{17} h\left(t_{\mathcal{G}^{\prime}}\right)+q_{18} .
$$

Démonstration. Supposons dans un premier temps que le plongement $\phi$ est construit à partir d'un fibré en droites très ample sur $\mathcal{A}$ comme expliqué dans l'appendice II de [39]. En notant $\mathcal{L}^{\prime}$ le groupe algébrique linéaire $\mathcal{G}^{\prime} \cap \mathcal{L}$, on a une suite exacte

$$
1 \rightarrow \mathcal{L}^{\prime} \stackrel{\iota}{\rightarrow} \mathcal{G}^{\prime} \stackrel{\pi}{\rightarrow} \mathcal{A}^{\prime} \rightarrow 1
$$

où $\mathcal{A}^{\prime}$ est un sous-groupe algébrique de $\mathcal{A}$. Comme $\mathcal{G}^{\prime}$ est connexe, $\mathcal{A}^{\prime}$ est connexe, donc c'est une sous-variété abélienne de $\mathcal{A}$. D'après [30, théorème 1], on a

$$
\operatorname{deg} \mathcal{G}^{\prime}=\left(\begin{array}{c}
\operatorname{dim} \mathcal{G}^{\prime} \\
\operatorname{dim} \mathcal{A}^{\prime}
\end{array}\right) \operatorname{deg} \mathcal{L}^{\prime} \operatorname{deg} \mathcal{A}^{\prime},
$$

d'où

$$
\operatorname{deg} \mathcal{L}^{\prime} \operatorname{deg} \mathcal{A}^{\prime} \leq \operatorname{deg} \mathcal{G}^{\prime} \leq 2^{\operatorname{dim} \mathcal{G}} \operatorname{deg} \mathcal{L}^{\prime} \operatorname{deg} \mathcal{A}^{\prime}
$$

Par ailleurs, la restriction de l'isomorphisme $\iota: t_{\mathcal{G}} / t_{\mathcal{L}} \rightarrow t_{\mathcal{A}}$ à $t_{\mathcal{G}^{\prime}} / t_{\mathcal{L}^{\prime}}$ fournit un isomorphisme $\iota_{\mid t_{\mathcal{G}^{\prime}} / t_{\mathcal{L}^{\prime}}}: t_{\mathcal{G}^{\prime}} / t_{\mathcal{L}^{\prime}} \rightarrow t_{\mathcal{A}^{\prime}}$. La structure de fibré adélique hermitien de $t_{\mathcal{G}}$ induit une structure de fibré adélique hermitien sur $t_{\mathcal{L}}$, puis sur $t_{\mathcal{A}}=t_{\mathcal{G}} / t_{\mathcal{L}}$ par quotient. D'après le lemme 2.10 page 14 , nous avons

$$
\widehat{\mu}_{\mathrm{n}}\left(t_{\mathcal{G}^{\prime}} / t_{\mathcal{L}^{\prime}}\right) \leq h\left(\iota_{\mid t_{\mathcal{G}^{\prime}} / t_{\mathcal{L}^{\prime}}}\right)+\widehat{\mu}_{\mathrm{n}}\left(t_{\mathcal{A}^{\prime}}\right) \leq h(\iota)+\widehat{\mu}_{\mathrm{n}}\left(t_{\mathcal{A}^{\prime}}\right)=h(\iota)-h\left(t_{\mathcal{A}^{\prime}}\right) / \operatorname{dim} \mathcal{A}^{\prime} .
$$

D'après la proposition 2.7 de la page 13 , on a également

$$
\widehat{\mu}_{\mathrm{n}}\left(t_{\mathcal{G}^{\prime}} / t_{\mathcal{L}^{\prime}}\right)=-h\left(t_{\mathcal{G}^{\prime}} / t_{\mathcal{L}^{\prime}}\right) / \operatorname{dim} t_{\mathcal{A}^{\prime}}=\left(-h\left(t_{\mathcal{G}^{\prime}}\right)+h\left(t_{\mathcal{L}^{\prime}}\right)\right) / \operatorname{dim} t_{\mathcal{A}^{\prime}} .
$$

En posant $c=\operatorname{dim} \mathcal{G} \max \{1, h(\iota)\}$, on a donc $h\left(t_{\mathcal{G}^{\prime}}\right)+c \geq h\left(t_{\mathcal{L}^{\prime}}\right)+h\left(t_{\mathcal{A}^{\prime}}\right)$. D'après les lemmes 5.7 et 5.9 , on en déduit qu'il existe des constantes $c^{\prime}$ et $c^{\prime \prime}$ ne dépendant que de $\mathcal{G}$ telles que

$$
\log \left(\operatorname{deg} \mathcal{G}^{\prime}\right) \leq c^{\prime} h\left(t_{\mathcal{G}^{\prime}}\right)+c^{\prime \prime},
$$

ce qui démontre la proposition 5.10 pour notre choix de $\phi$. Comme un changement de choix de plongement ne modifie le degré qu'à constante près (voir [3, remarque 2]), la proposition est démontrée.

5.4. Démonstration du théorème 5.2. Nous avons déjà démontré les points (2) et (3) $\mathrm{du}$ théorème 5.2 au paragraphe 5.1. Montrons le premier point. Soit $\mathcal{G}^{\prime}$ un sous-groupe algébrique connexe de $\mathcal{G}$ tel que $t_{\mathcal{G}^{\prime}}+t_{\mathcal{H}} \neq t_{\mathcal{G}}$. Le groupe $\mathcal{G}^{\prime}+\mathcal{H}=m\left(\mathcal{G}^{\prime} \times{ }_{\overline{\mathbb{Q}}} \mathcal{H}\right.$ ) (où $m$ désigne la loi de $\mathcal{G}$ ) vérifie $t_{\mathcal{G}^{\prime}+\mathcal{H}}=t_{\mathcal{G}^{\prime}}+t_{\mathcal{H}}$ d'après le lemme 5.5 . On en déduit que $t_{\mathcal{G}^{\prime}+\mathcal{H}}+t_{\mathcal{H}}=t_{\mathcal{G}^{\prime}}+t_{\mathcal{H}} \neq t_{\mathcal{G}}$. En appliquant l'hypothèse du théorème 5.2 $[1]$ à $\mathcal{G}^{\prime}+\mathcal{H}$, on 
a $\mathrm{u} \notin t_{\mathcal{G}^{\prime}+\mathcal{H}}\left(\mathbb{C}_{v}\right)$. Par inclusion de $t_{\mathcal{H}}\left(\mathbb{C}_{v}\right)$ dans $t_{\mathcal{G}^{\prime}+\mathcal{H}}\left(\mathbb{C}_{v}\right)=t_{\mathcal{G}^{\prime}}\left(\mathbb{C}_{v}\right)+t_{\mathcal{H}}\left(\mathbb{C}_{v}\right)$, on a par ailleurs $\mathrm{d}_{v}\left(\mathrm{u}, t_{\mathcal{G}^{\prime}+\mathcal{H}}\left(\mathbb{C}_{v}\right)\right) \leq \mathrm{d}_{v}\left(\mathrm{u}, t_{\mathcal{H}}\left(\mathbb{C}_{v}\right)\right)$. D'après le lemme 5.6, on a

$$
\mathrm{d}_{v}\left(\mathrm{u}, t_{\mathcal{G}^{\prime}+\mathcal{H}}\left(\mathbb{C}_{v}\right)\right) \leq \mathrm{d}_{v}\left(\mathrm{u}, t_{\mathcal{H}}\left(\mathbb{C}_{v}\right)\right)<\frac{1}{q_{8} S \operatorname{deg} \mathcal{G}^{\prime} \operatorname{deg} \mathcal{H}}<\frac{1}{q_{10} S \operatorname{deg}\left(\mathcal{G}^{\prime}+\mathcal{H}\right)}
$$

pourvu que la constante $q_{8}$ soit suffisamment grande. En appliquant le lemme 5.4 à $\mathcal{A}=\mathcal{G}^{\prime}+\mathcal{H}$, on en déduit que $s \mathrm{p} \notin\left(\mathcal{G}^{\prime}+\mathcal{H}\right)(\overline{\mathbb{Q}})$ pour tout $s \in\{1, \ldots, S\}$, donc sp $\notin$ $\mathcal{G}^{\prime}(\overline{\mathbb{Q}}) \subset\left(\mathcal{G}^{\prime}+\mathcal{H}\right)(\overline{\mathbb{Q}})$. Le point $(4)$ du théorème 5.2 est une conséquence immédiate des deux premiers points et de la proposition 5.10 .

\section{Lemmes d'interpolation}

Dans la démonstration des théorèmes du paragraphe 4, nous aurons besoin de lemmes d'interpolation (à l'étape d'extrapolation, 88.8). Nous utiliserons un résultat différent selon que la place considérée est archimédienne ou ultramétrique. Dans le premier cas, nous appliquerons un résultat classique dû à Cijsouw et Waldschmidt [12. On pourra consulter l'article de Bertrand [2] pour un exposé sur les lemmes d'interpolation ultramétriques. Dans cet article, nous ferons appel à un cas très particulier d'un théorème de Robba [36, remarque iii) page 276]. Plus précisément, nous allons montrer que les arguments de Robba fournissent un résultat légèrement plus général que celui énoncé dans [36].

Si $L$ est un corps valué, alors pour tout $a \in L$ et pour tout nombre réel $r>0$, on note

$$
D_{L}(a, r):=\{z \in L|| z-a \mid \leq r\}
$$

le disque ouvert de centre $a$ et de rayon $r$.

6.1. Cas d'une place archimédienne. Le lemme d'interpolation suivant est un corollaire de [12, lemme 2] (voir aussi [40, lemme 2.3] et [34, proposition 4.2]).

Lemme 6.1. Soient $T_{1}, S_{1}$ des entiers strictement positifs et soient $R \geq r \geq 2 S_{1}$ des nombres réels. Pour toute fonction $f: \mathbb{C} \rightarrow \mathbb{C}$ analytique dans le disque $D_{\mathbb{C}}(0, R)$, on a

$$
|f|_{r} \leq 2|f|_{R}\left(\frac{2 r}{R}\right)^{T_{1} S_{1}}+5\left(\frac{9 r}{S_{1}}\right)^{T_{1} S_{1}} \max _{\substack{t \in \mathbb{N}, t<S_{1} \\ h \leq T_{1}}}\left|\frac{f^{(h)}}{h !}(t)\right|_{v_{0}}
$$

où $|f|_{\zeta}:=\sup \{|f(z)||| z \mid \leq \zeta\}$ pour tout $\zeta>0$.

Signalons que des lemmes d'interpolations plus précis ont été démontrés depuis [12] (voir par exemple [6, proposition 2.1]). Comme nous n'expliciterons pas les constantes qui interviendront dans le résultat final, nous nous contenterons de la version (plus simple) présentée ci-dessus.

6.2. Cas d'une place finie. Soit $L$ un corps valué ultramétrique localement compact de caractéristique nulle et soit $L^{\prime}$ une extension valuée de $L$. Si $a \in L^{\prime}$ et si $R>0$ est un nombre réel, nous dirons qu'une fonction $f: L^{\prime} \rightarrow L^{\prime}$ est analytique sur le disque 
$D_{L^{\prime}}(a, R):=\left\{z \in L^{\prime}|| a-z \mid \leq R\right\}$ si pour tout $z \in D_{L^{\prime}}(a, R)$, on a le développement

$$
f(z)=\sum_{\ell \in \mathbb{N}} \frac{f^{(\ell)}(a)}{\ell !}(z-a)^{\ell}
$$

avec

$$
\lim _{\ell \rightarrow+\infty}\left|\frac{f^{(\ell)}(a)}{\ell !}\right| R^{\ell}=0 .
$$

Soit $f: L^{\prime} \rightarrow L^{\prime}$ une fonction analytique sur le disque $D_{L^{\prime}}(a, R)$ et soit $r \leq R$. Notons

$$
|f|_{a}(r)=\max _{\ell \in \mathbb{N}}\left|\frac{f^{(\ell)}(a)}{\ell !}\right| r^{\ell} .
$$

Si $\widehat{L}$ désigne le complété d'une clôture algébrique de $L^{\prime}$, la fonction $f$ s'étend naturellement à $D_{\widehat{L}}(a, r)$, et l'inégalité de Cauchy donne

$$
|f|_{a}(r)=\sup \left\{|f(z)| \mid z \in D_{\widehat{L}}(a, r)\right\} .
$$

En particulier, pour tout $\nu \in D_{L^{\prime}}(a, r)$, on a $|f|_{a}(r)=|f|_{\nu}(r)$. En suivant Robba [36, $\S 1.1 .1]$, nous définissons l'entier $N_{a}(f, r)$ (respectivement $\left.n_{a}(f, r)\right)$ comme le maximum (respectivement le minimum) de l'ensemble non vide

$$
\left\{\left.\ell \in \mathbb{N}|| \frac{f^{(\ell)}(a)}{\ell !}\left|r^{\ell}=\right| f\right|_{a}(r)\right\} \text {. }
$$

La fonction $\log (r) \mapsto \log |f|_{a}(r)$, définie pour $\log r \leq \log R$, est continue, affine par morceaux, et sa dérivée à droite (respectivement à gauche) au point $\log r$ est $N_{a}(f, r)$ (respectivement $n_{a}(f, r)$ ). De plus, le nombre $N_{a}(f, r)$ (respectivement $n_{a}(f, r)$ ) est égal au nombre de zéros $z$ de $f$ comptés avec multiplicités tels que $|z-\nu| \leq \rho$ (respectivement $|z-\nu|<\rho$ ) dans une clôture algébrique de $L$ (voir [36, remarque page 255]).

Notons $q$ le cardinal du corps de restes et $\lambda$ la valeur absolue d'une uniformisante de $L$ (ainsi, $L$ est de type $(q, \lambda)$ au sens de [36, §2.6]). Soit $r$ un nombre réel appartenant au groupe de valuation de $L$ et soit $\left(u_{i}\right)_{i \in \mathbb{N}} \in D_{L}(0, r)^{\mathbb{N}}$ une suite très bien répartie dans $D_{L}(0, r)$ au sens d'Amice [1] : pour tout $\nu \in D_{L}(0, r)$ et pour tous entiers naturels $h, k$, on a l'encadrement

$$
\left[\frac{h}{q^{k}}\right] \leq \operatorname{card}\left\{i \in \mathbb{N}|i<h,| u_{i}-\nu \mid \leq r \lambda^{k}\right\} \leq\left[\frac{h-1}{q^{k}}\right]+1 .
$$

ThÉORÈme 6.2. Soit $R>r$ et soit $L^{\prime}$ une extension valuée de L. Considérons une fonction $f: L^{\prime} \rightarrow L^{\prime}$ analytique sur $D_{L^{\prime}}(0, R)=\left\{z \in L^{\prime}|| z \mid \leq R\right\}$. Soient $S_{1} \geq 2$, $T_{1} \geq 1$ deux nombres entiers. On pose

$$
\delta=\min \left\{\left|u_{i}-u_{j}\right| \mid i, j<S_{1}\right\} .
$$

Alors pour tout entier $n \in \mathbb{N}$ et pour tout $\nu \in D_{L}(0, r)$, on a

$$
\left|\frac{f^{(n)}(\nu)}{n !}\right| \leq \max \left\{r^{-n}(r / R)^{S_{1} T_{1}}|f|_{0}(R), \delta^{-n}(r / \delta)^{T_{1}-1} \max _{\substack{h, k \in \mathbb{N} \\ h<T_{1}, k<S_{1}}}\left|\frac{f^{(h)}\left(u_{k}\right)}{h !}\right| \delta^{h}\right\} .
$$

Démonstration. Le théorème correspond à la remarque iii) page 276 de [36] appliquée à $h=S_{1}, k=T_{1}, K=L$. Il est important de signaler que le théorème page 275 de [36] reste valable en remplaçant l'hypothèse $« f: K \rightarrow K$ analytique $»$ par $« f: \widetilde{K} \rightarrow \widetilde{K}$ 
analytique $»$, où $\widetilde{K}$ désigne un corps valué contenant $K$. En effet, ce théorème repose sur celui de la page 270 (qui n'impose pas d'hypothèse sur $K$ ), et sur les majorations des quantités $\varepsilon_{\gamma}(f)$ et $c_{\gamma, z}^{\prime}$ de [36, §5.4]. Le point est que $c_{\gamma, z}^{\prime}$ ne dépend pas de $f$, et que l'on peut donc utiliser l'inégalité (5.4.10) page $275 \mathrm{du}$ moment que $K$ est localement compact et que la suite $\left(u_{i}\right)_{i \in \mathbb{N}}$ est très bien répartie dans une boule de $K$. La majoration de $\varepsilon_{\gamma}(f)$ est quant à elle inchangée si l'on considère une extension valuée du corps $K$. Pour le confort du lecteur, nous allons rappeler le raisonnement de Robba.

Considérons l'ensemble $\Gamma:=\left\{i \in \mathbb{N} \mid 0 \leq i<S_{1}\right\}$. En notant $s$ l'entier tel que $q^{s-1}<S_{1} \leq q^{s}$, on a $\delta=r \lambda^{s-1}$. Considérons le polynôme $P(X)=\prod_{i=0}^{S_{1}-1}\left(X-u_{i}\right)^{T_{1}}$; pour tout $i \in \Gamma$, posons $P_{i}(X)=P(X) /\left(X-u_{i}\right)$ et $\delta_{i}=\min \left\{\left|u_{i}-u_{j}\right| \mid 0 \leq j<S_{1}, j \neq i\right\}$. Si $g: L^{\prime} \rightarrow L^{\prime}$ est une fonction analytique sur $D_{L^{\prime}}(0, R)$ et si $i \in \Gamma$, on note également

$$
\varepsilon_{i}(g)=\max \left\{\left|\frac{g^{(h)}\left(u_{i}\right)}{h !}\right| \delta_{i}^{h} \mid h \in \mathbb{N}, h<T_{1}\right\} .
$$

Soit $\nu \in D_{L}(0, r)$ et soit $0 \leq i<S_{1}$ tel que $\left|\nu-u_{i}\right|=\min \left\{\left|\nu-u_{j}\right| \mid 0 \leq j<S_{1}\right\}$. On pose alors $\delta_{\nu}=\max \left\{\left|\nu-u_{i}\right|, \delta_{i}\right\}$. L'encadrement (6.1) entraîne que pour tout $\nu \in D_{L}(0, r)$, la quantité $\delta_{\nu}$ vaut $\delta$ ou $\delta / \lambda$ (voir [36, (5.4.2)]). Pour tout $0 \leq i<S_{1}$ et pour tout $\nu \in D_{L}(0, r)$, on pose

$$
c_{i, \nu}^{\prime}=\left|P_{i}\right|_{\nu}\left(\delta_{\nu}\right) / \varepsilon_{i}\left(P_{i}\right) .
$$

Soit $\nu \in D_{L}(0, r)$. D'après [36, théorème page 270] (appliqué à $m\left(u_{i}\right)=T_{1}$ pour tout $i \in \Gamma)$, on a

$$
\begin{cases}|f|_{\nu}(r)=|f|_{0}(r) \leq(r / R)^{S_{1} T_{1}}|f|_{0}(R) & \text { si } N_{0}(f, r) \geq S_{1} T_{1} \\ |f|_{\nu}(\delta) \leq|f|_{\nu}\left(\delta_{\nu}\right) \leq \max _{i \in \Gamma} c_{i, \nu}^{\prime} \varepsilon_{i}(f) & \text { sinon. }\end{cases}
$$

Posons

$$
\varepsilon=\max _{\substack{h, k \in \mathbb{N} \\ h<S_{1}, k<T_{1}}}\left|\frac{f^{(h)}\left(u_{k}\right)}{h !}\right| \delta^{h} .
$$

Soit $i \in \Gamma$. Il découle immédiatement de la définition de $\varepsilon_{i}(f)$ que

$$
\begin{cases}\varepsilon_{i}(f) \leq \varepsilon & \text { si } \delta_{i}=\delta \\ \varepsilon_{i}(f) \leq \varepsilon \lambda^{-\left(T_{1}-1\right)} & \text { si } \delta_{i}=\delta / \lambda\end{cases}
$$

Soit $\nu \in D_{L}(0, r)$. Nous allons maintenant majorer la quantité $c_{i, \nu}^{\prime}$. Commençons par montrer la propriété suivante : pour tous entiers naturels $m \leq n$,

$$
\log _{\lambda}\left(\left|P_{i}\right|_{\nu}\left(r \lambda^{m}\right)\right)-\log _{\lambda}\left(\left|P_{i}\right|_{\nu}\left(r \lambda^{n}\right)\right)=-\sum_{j=m+1}^{n} N_{\nu}\left(P_{i}, r \lambda^{j}\right) .
$$

En utilisant la description de $N_{\nu}\left(P_{i}, \rho\right)$ et $n_{\nu}\left(P_{i}, \rho\right)$ en termes de zéros de $P_{i}$, on a

$$
N_{\nu}\left(P_{i}, \rho\right)= \begin{cases}T_{1} \operatorname{card}\left\{0 \leq j<S_{1}|| u_{j}-\nu \mid \leq \rho\right\} & \text { si } \rho<\left|u_{i}-\nu\right| \\ T_{1} \operatorname{card}\left\{0 \leq j<S_{1}|| u_{j}-\nu \mid \leq \rho\right\}-1 & \text { si } \rho \geq\left|u_{i}-\nu\right|\end{cases}
$$

et

$$
n_{\nu}\left(P_{i}, \rho\right)= \begin{cases}T_{1} \operatorname{card}\left\{0 \leq j<S_{1}|| u_{j}-\nu \mid<\rho\right\} & \text { si } \rho \leq\left|u_{i}-\nu\right| \\ T_{1} \operatorname{card}\left\{0 \leq j<S_{1}|| u_{j}-\nu \mid<\rho\right\}-1 & \text { si } \rho>\left|u_{i}-\nu\right|\end{cases}
$$


Pour tout $\rho \in] r \lambda^{m+1}, r \lambda^{m}[$, on a

$\left\{0 \leq j<S|| u_{j}-\nu \mid<\rho\right\}=\left\{0 \leq j<S|| u_{j}-\nu \mid \leq \rho\right\}=\left\{0 \leq j<S|| u_{j}-\nu \mid \leq r \lambda^{m+1}\right\}$, et donc $N_{\nu}\left(P_{i}, \rho\right)=n_{\nu}\left(P_{i}, \rho\right)=N_{\nu}\left(P_{i}, r \lambda^{m+1}\right)$. En particulier, la fonction

$$
F: \log \xi \mapsto \log \left|P_{i}\right|_{\nu}(\xi)
$$

est dérivable sur l'intervalle $] \log \left(r \lambda^{m+1}\right), \log \left(r \lambda^{m}\right)[$, de dérivée constante

$$
F^{\prime}(\log \xi)=N_{\nu}\left(P_{i}, \xi\right)=N_{\nu}\left(P_{i}, r \lambda^{m+1}\right) .
$$

On en déduit que

$$
\left(\log \left(r \lambda^{m}\right)-\log \left(r \lambda^{m+1}\right)\right) N_{\nu}\left(P_{i}, r \lambda^{m+1}\right)=F\left(\log \left(r \lambda^{m}\right)\right)-F\left(\log \left(r \lambda^{m+1}\right)\right),
$$

et donc

$$
-\log (\lambda) N_{\nu}\left(P_{i}, \lambda^{m+1}\right)=\log \left|P_{i}\right|_{\nu}\left(r \lambda^{m}\right)-\log \left|P_{i}\right|_{\nu}\left(r \lambda^{m+1}\right) .
$$

L'égalité 6.4 est donc démontrée dans le cas où $n=m+1$, et l'on en déduit immédiatement le cas général. D'après (6.6), on a par ailleurs $n_{u_{i}}\left(P_{i}, \delta_{i}\right)=T_{1}-1$, donc

$$
\left|P_{i}\right|_{u_{i}}\left(\delta_{i}\right)=\sup _{h \in \mathbb{N}}\left|\frac{P_{i}^{(h)}\left(u_{i}\right)}{h !}\right| \delta_{i}^{h}=\sup _{h<T_{1}}\left|\frac{P_{i}^{(h)}\left(u_{i}\right)}{h !}\right| \delta_{i}^{h}=\varepsilon_{i}\left(P_{i}\right) .
$$

On a ainsi

$$
\begin{aligned}
\log _{\lambda} c_{i, \nu}^{\prime} & =\log _{\lambda}\left(\left|P_{i}\right|_{\nu}\left(\delta_{\nu}\right)\right)-\log _{\lambda}\left(\varepsilon_{i}\left(P_{i}\right)\right) \\
& =\log _{\lambda}\left(\left|P_{i}\right|_{\nu}\left(\delta_{\nu}\right)\right)-\log _{\lambda}\left(\left|P_{i}\right|_{u_{i}}\left(\delta_{i}\right)\right) \\
& =\log _{\lambda}\left(\left|P_{i}\right|_{\nu}\left(r \lambda^{s_{\nu}}\right)\right)-\log _{\lambda}\left(\left|P_{i}\right|_{u_{i}}\left(r \lambda^{s_{i}}\right)\right),
\end{aligned}
$$

où $s_{\nu}, s_{i} \in\{s-1, s-2\}$ sont les entiers tels que $\delta_{\nu}=r \lambda^{s_{\nu}}$ et $\delta_{i}=r \lambda^{s_{i}}$. Si $\left|\nu-u_{i}\right| \leq \delta_{i}$, on a $\left|P_{i}\right|_{u_{i}}\left(\delta_{i}\right)=\left|P_{i}\right|_{\nu}\left(\delta_{i}\right)$ et $\delta_{i}=\delta_{\nu}$, donc $c_{i, \nu}^{\prime} \leq 1$. Supposons que $\left|\nu-u_{i}\right|>\delta_{i}$ et soit $1 \leq \sigma \leq s_{i} \leq s_{\nu}$ l'entier tel que

$$
r \lambda^{\sigma}<\left|\nu-u_{i}\right| \leq r \lambda^{\sigma-1}
$$

D'après 6.1), 6.4 et 6.5), on a

$$
\begin{aligned}
& \log _{\lambda}\left(\left|P_{i}\right|_{\nu}\left(r \lambda^{s_{\nu}}\right)\right)-\log _{\lambda}\left(\left|P_{i}\right|_{\nu}\left(r \lambda^{\sigma-1}\right)\right) \geq T_{1} \sum_{j=\sigma}^{s_{\nu}}\left[\frac{S_{1}}{q^{j}}\right] \geq T_{1} \sum_{j=\sigma}^{s_{i}}\left[\frac{S_{1}}{q^{j}}\right], \\
& \log _{\lambda}\left(\left|P_{i}\right|_{u_{i}}\left(r \lambda^{\sigma-1}\right)\right)-\log _{\lambda}\left(\left|P_{i}\right|_{u_{i}}\left(r \lambda^{s_{i}}\right)\right) \geq-\sum_{j=\sigma}^{s_{i}}\left(T_{1}\left(\left[\frac{S_{1}-1}{q^{j}}\right]+1\right)-1\right) .
\end{aligned}
$$

Comme $\left|\nu-u_{i}\right| \leq r \lambda^{\sigma-1}$, on a $\left|P_{i}\right|_{\nu}\left(r \lambda^{\sigma-1}\right)=\left|P_{i}\right|_{u_{i}}\left(r \lambda^{\sigma-1}\right)$, et on obtient

$$
\begin{aligned}
\log _{\lambda} c_{i, \nu}^{\prime} & \geq T_{1} \sum_{j=\sigma}^{s_{i}}\left[\frac{S_{1}}{q^{j}}\right]-\sum_{j=\sigma}^{s_{i}}\left(T_{1}\left(\left[\frac{S_{1}-1}{q^{j}}\right]+1\right)-1\right) \\
& =-\sum_{j=\sigma}^{s_{i}}\left(T_{1}\left(\left[\frac{S_{1}-1}{q^{j}}\right]+1-\left[\frac{S_{1}}{q^{j}}\right]\right)-1\right) \\
& \geq-s_{i}\left(T_{1}-1\right) .
\end{aligned}
$$


Par ailleurs, $s_{i}=s-1$ si $\delta_{i}=\delta$ et $s_{i}=s-2$ si $\delta_{i}=\delta / \lambda$. On a donc

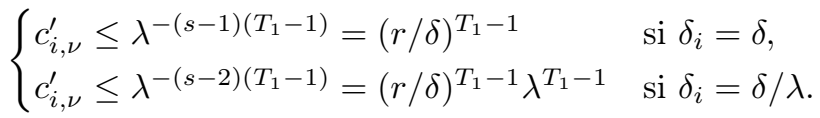

D'après 6.3 et 6.8, pour tout $i<S_{1}$ et tout $\nu \in D_{L}(0, r)$, on a $c_{i, \nu}^{\prime} \varepsilon_{i}(f) \leq(r / \delta)^{T_{1}-1} \varepsilon$. En appliquant (6.2), on en déduit que pour tout entier $n \in \mathbb{N}$ et tout $\nu \in D_{L}(0, r)$,

$$
\begin{aligned}
\left|\frac{f^{(n)}(\nu)}{n !}\right| & \leq \max \left\{r^{-n}|f|_{\nu}(r), \delta^{-n}|f|_{\nu}\left(\delta_{\nu}\right)\right\} \\
& \leq \max \left\{r^{-n}(r / R)^{S_{1} T_{1}}|f|_{0}(R), \delta^{-n}(r / \delta)^{T_{1}-1} \varepsilon\right\},
\end{aligned}
$$

ce qui achève la preuve.

Nous appliquerons le théorème 6.2 dans la situation du corollaire suivant.

Corollaire 6.3. Soit $L$ un corps de nombres et soit $v$ une place de $L$ au-dessus d'un nombre premier $p$. Soit $R \geq 1$ un nombre réel et soient $S_{1} \geq 2, T_{1} \geq 1$ deux nombres entiers. Soit

$$
f:\left\{\left.z \in \mathbb{C}_{v}|| z\right|_{v} \leq R\right\} \rightarrow \mathbb{C}_{v}
$$

une fonction analytique. Alors pour tout $\nu \in \mathbb{Z}_{p}$, on a

$$
|f(\nu)|_{v} \leq \max \left\{\left(\frac{1}{R}\right)^{T_{1} S_{1}}|f|_{v, R}, \gamma \max _{\substack{h, k \in \mathbb{N} \\ h<T_{1}, k<S_{1}}}\left|\frac{f^{(h)}(k)}{h !}\right|_{v}\right\},
$$

où

$$
\gamma=p^{\left(T_{1}-1\right)\left[\log \left(S_{1}-1\right) / \log p\right]}, \quad|f|_{v, R}=\sup \left\{\left.|f(z)|_{v}\left|z \in \mathbb{C}_{v},\right| z\right|_{v} \leq R\right\} .
$$

Démonstration. Le corps $\mathbb{Q}_{p}$ est localement compact de type $(q, \lambda)=(p, 1 / p)$ au sens de [36, §2.6]. Par ailleurs, la suite des entiers naturels est très bien répartie dans $\mathbb{Z}_{p}=$ $\left\{\left.\nu \in \mathbb{Q}_{p}|| \nu\right|_{p} \leq 1\right\}$ (voir [1, §2.1 et exemple 2.3.1] et [36, §5.4]). De plus, on a

$$
\delta=\min \left\{|j-k|_{p} \mid j, k \in\left\{0, \ldots, S_{1}-1\right\}, j \neq k\right\}=p^{-\left[\log \left(S_{1}\right) / \log p\right]} .
$$

La conclusion du corollaire 6.3 est triviale si $R=1$. Si $R>1$, c'est une conséquence immédiate du théorème 6.2 appliqué à $n=0, r=1, L=\mathbb{Q}_{p}, L^{\prime}=\mathbb{C}_{v}$, et à la suite $u_{i}=i$ des entiers naturels.

\section{Compléments sur l'exponentielle}

Reprenons les notations du paragraphe 3. Soit $v$ une place quelconque de $K$ et soit $i \in\{1, \ldots, n\}$. Rappelons que l'on a fixé des fonctions $\varphi_{v, i, 0}, \ldots, \varphi_{v, i, N_{i}}$, analytiques de $\mathscr{U}_{v, i} \subseteq t_{G_{i}}\left(\mathbb{C}_{v}\right)$ dans $\mathbb{C}_{v}$, telles que pour tout $z \in \mathscr{U}_{v, i}$, on ait

$$
\exp _{v, i}(z)=\left(\varphi_{v, i, 0}(z): \cdots: \varphi_{v, i, N_{i}}(z)\right)
$$

(voir page 16). Nous noterons

$$
\Psi_{v, i}: \mathscr{U}_{v, i} \ni z \mapsto\left(\varphi_{v, i, 0}(z), \cdots, \varphi_{v, i, N_{i}}(z)\right) \in \mathbb{C}_{v}^{N_{i}+1},
$$


et pour $j \in\left\{0, \ldots, N_{i}\right\}$,

$$
\Theta_{v, i, j}: \mathscr{U}_{v, i} \backslash \varphi_{v, i, j}^{-1}(\{0\}) \ni z \mapsto\left(\frac{\varphi_{v, i, 0}}{\varphi_{v, i, j}}(z), \ldots, \frac{\varphi_{v, i, N_{i}}}{\varphi_{v, i, j}}(z)\right) \in \mathbb{C}_{v}^{N_{i}+1}
$$

Enfin, posons

$$
\Psi_{v}=\Psi_{v, 1} \times \cdots \times \Psi_{v, n}: \mathscr{U}_{v} \rightarrow \mathbb{C}_{v}^{N_{1}+1} \times \cdots \times \mathbb{C}_{v}^{N_{n}+1} .
$$

D'après [41, propriété 4.6], les anneaux

$$
K\left[\left(\varphi_{v, i, j} / \varphi_{v, i, k}\right)_{0 \leq j \leq N_{i}}\right], \quad 0 \leq k \leq N_{i},
$$

sont stables par dérivation selon un vecteur du $K$-espace vectoriel $t_{G_{i}}(K)$ pour tout $i \in\{1, \ldots, n\}$ et pour toute place $v$ de $K$. Cette propriété nous sera utile au 8.7

Plongement et loi d'addition. Soit $i \in\{1, \ldots, n\}$. Soit $x \in \mathscr{U}_{v_{0}, i} \subset t_{G_{i}}\left(\mathbb{C}_{v_{0}}\right)$. D'après [41, propriété 4.4] (voir aussi [21, page 229]), il existe une constante q19 $\geq 1$ et une famille de polynômes $\left(A_{x, j}^{(i)}(\mathbf{X}, \mathbf{Y})\right)_{0 \leq j \leq N_{i}}$ de l'anneau $K\left[X_{0}, \ldots, X_{N_{i}}, Y_{0}, \ldots, Y_{N_{i}}\right]$ telle que :

- chacun des $A_{x, j}^{(i)}(\mathbf{X}, \mathbf{Y}), 0 \leq j \leq N_{i}$, est homogène de même degré, inférieur à q19, en chacune des variables $\mathbf{X}=\left(X_{0}, \ldots, X_{N_{i}}\right)$ et $\mathbf{Y}=\left(Y_{0}, \ldots, Y_{N_{i}}\right)$;

- pour tout $z \in \mathscr{U}_{v_{0}, i}$ au voisinage de $x$, on a l'égalité

$$
\exp _{v_{0}, i}(x+z)=\left(A_{x, 0}^{(i)}\left(\Psi_{v_{0}, i}(x), \Psi_{v_{0}, i}(z)\right): \cdots: A_{x, N_{i}}^{(i)}\left(\Psi_{v_{0}, i}(x), \Psi_{v_{0}, i}(z)\right)\right) .
$$

De plus, cette constante q19 peut être choisie uniforme en l'indice $i$ ainsi qu'en $x$ par quasi-compacité des groupes $G_{i}$. Pour tout $i \in\{1, \ldots, n\}$, on note

$$
A_{x}^{(i)}(\mathbf{X}, \mathbf{Y}):=\left(A_{x, 0}^{(i)}(\mathbf{X}, \mathbf{Y}): \cdots: A_{x, N_{i}}^{(i)}(\mathbf{X}, \mathbf{Y})\right)
$$

Afin d'alléger les notations, nous omettrons souvent la référence à $x$ dans cette notation, en écrivant $A_{j}^{(i)}$ au lieu de $A_{x, j}^{(i)}$ s'il n'y a pas d'ambiguïté.

Ordre analytique de l'exponentielle. Si $v_{0}$ est archimédienne, alors les fonctions $\varphi_{v_{0}, i, j}, 0 \leq j \leq N_{i}$, sont d'ordre analytique inférieur à $\rho_{i}$, où $\rho_{i}=1$ si $G_{i}$ est linéaire et $\rho_{i}=2$ sinon. De plus, d'après [41, page 75], il existe une constante $q_{20} \geq 1$ telle que pour tout entier $i \in\{1, \ldots, n\}$ et tout vecteur $z \in t_{G_{i}}\left(\mathbb{C}_{v_{0}}\right)$, on ait

$$
-q_{20}\left(1+\|z\|_{v_{0}}\right)^{\rho_{i}} \leq \log \max _{0 \leq j \leq N_{i}}\left|\varphi_{v_{0}, i, j}(z)\right|_{v_{0}} \leq q_{20}\left(1+\|z\|_{v_{0}}\right)^{\rho_{i}} .
$$

Convention sur les notations. À l'exception du 8.7 , nous travaillerons exclusivement avec l'exponentielle $v_{0}$-adique $\exp _{v_{0}}: t_{G}\left(\mathbb{C}_{v_{0}}\right) \rightarrow G\left(\mathbb{C}_{v_{0}}\right)$. Afin de ne pas alourdir les notations, nous ne soulignerons plus la dépendance en la place $v_{0}$ dans les notations introduites précédemment, et écrivant plus simplement $\mathscr{U}$, exp, $\left(\Psi_{i}\right)_{1 \leq i \leq n}$, etc.

\section{Démonstration dans le cas semi-abélien}

Nous allons commencer par démontrer les théorèmes 4.3 et 4.4 , qui concernent le cas semi-abélien. On désigne par $G_{0}$ le groupe additif $\mathbb{G}_{a}$ (plongé dans $\mathbb{P}_{\overline{\mathbb{Q}}}^{1}$ par $z \mapsto(1: z)$ ) et on note $W$ le sous- $K$-espace vectoriel $t_{G_{0}}(K) \oplus V=K \oplus V$ de $t_{G_{0} \times G}(K)=K \oplus t_{G}(K)$. On pose $e_{0}=1 \in t_{G_{0}}(K)=K$. L'espace vectoriel $t_{G_{0} \times G}(K)$ est muni d'une structure 
de fibré adélique hermitien par somme directe. Par abus de notation, nous notons encore $d(\cdot, \cdot)$ la distance associée à la norme $\|\cdot\|_{v_{0}}$ sur $t_{G_{0} \times G}\left(\mathbb{C}_{v_{0}}\right)$. Remarquons que $d(u, V)=$ $d((1, u), W)$ et d'après la proposition 2.7, on a également $h(W)=h(V)$. Enfin, on note $\mathbf{q}=(1, \mathbf{p}) \in\left(G_{0} \times G\right)(K)$. À partir de maintenant, nous faisons l'hypothèse suivante :

Hypothèse 1. Pour tout sous-groupe algébrique connexe $G^{\prime}$ de $G$ tel que $t_{G^{\prime}}+V \neq t_{G}$, on a $u \notin t_{G^{\prime}}\left(\mathbb{C}_{v_{0}}\right)$.

Par abus de notation, nous avons confondu le $K$-espace vectoriel $V$ avec $V \otimes_{K} \overline{\mathbb{Q}}=t_{H}$ dans l'écriture $t_{G^{\prime}}+V \neq t_{G}$. Ainsi, l'expression $« t_{G^{\prime}}+V \neq t_{G} »$ signifie $《 t_{G^{\prime}}+V \otimes \overline{\mathbb{Q}} \neq$ $t_{G} \gg$. Afin de ne pas alourdir la démonstration, nous reproduirons cet abus régulièrement dans la suite du texte.

Cette hypothèse implique en particulier que le point $u$ n'appartient pas à $V \otimes_{K} \mathbb{C}_{v_{0}}$. On suppose que le groupe $G$ est une variété semi-abélienne. L'hypothèse 1 entraîne alors que pour tout sous-groupe algébrique connexe $G^{\prime}$ de $G_{0} \times \overline{\mathbb{Q}} G$ tel que $t_{G^{\prime}}+W \neq t_{G_{0} \times G}$, on a $(1, u) \notin t_{G^{\prime}}\left(\mathbb{C}_{v_{0}}\right)$. En effet, comme $G$ est semi-abélien, tout sous-groupe $G^{\prime} \operatorname{de} G_{0} \times G$ s'écrit sous la forme $G^{\prime}=G_{0}^{\prime} \times G^{\prime \prime}$, où $G_{0}^{\prime}$ est un sous-groupe algébrique de $G_{0}$ et $G^{\prime \prime}$ est un sous-groupe algébrique de $G$. Si $(1, u) \in t_{G^{\prime}}\left(\mathbb{C}_{v_{0}}\right)$, alors $u \in t_{G^{\prime \prime}}\left(\mathbb{C}_{v_{0}}\right)$, et d'après l'hypothèse 1 , on a

$$
t_{G^{\prime}}+W=t_{G_{0}} \oplus\left(t_{G^{\prime \prime}}+V\right)=t_{G_{0}} \oplus t_{G}=t_{G_{0} \times G} .
$$

8.1. Choix d'un sous-groupe. L'objet de ce paragraphe est de choisir un sous-groupe particulier $\widetilde{G}$ de $G_{0} \times_{\overline{\mathbb{Q}}} G$, qui jouera un rôle fondamental dans la construction d'une section auxiliaire (voir les paragraphes 8.5.1 et 8.6). Les définitions qui suivent correspondent à celles de [21, §3.2]. Une nouveauté cruciale pour la suite de la démonstration est le lemme 8.3, qui repose sur les résultats du paragraphe 5, et qui nous permettra d'exclure le cas dit « périodique », où des multiples de $\mathbf{q}$ appartiennent à $\widetilde{G}$.

Soient $\widetilde{D}_{0}, \widetilde{D}_{1}, \ldots, \widetilde{D}_{n}, \widetilde{T}, C_{0}$ des nombres réels strictement positifs et $0<S_{0}<S$ des entiers. On suppose que $\widetilde{T}>1$ et on pose $T=[\widetilde{T}]$. Dans toute la suite, si $G^{\prime}$ est un sousgroupe algébrique de $G_{0} \times \overline{\mathbb{Q}} G$, on note $r^{\prime}=\operatorname{codim}_{G_{0} \times G} G^{\prime}$ et $\lambda^{\prime}=\operatorname{codim}_{W}\left(t_{G^{\prime}} \cap W\right)$ (où, par abus de notation, nous avons confondu $W$ avec $W \otimes \overline{\mathbb{Q}})$. De plus, posons $\Sigma_{\mathbf{q}}(S)=$ $\left\{0_{G_{0} \times G}, \mathbf{q}, \ldots, S \mathbf{q}\right\}$.

DÉFInItion 8.1. Soit $G^{\prime}$ un sous-groupe algébrique connexe de $G_{0} \times_{\overline{\mathbb{Q}}} G$ tel que $t_{G^{\prime}}+W \neq$ $t_{G_{0} \times G}$. On définit le réel strictement positif

$$
A\left(G^{\prime}\right)=\left(\frac{\widetilde{T}^{\lambda^{\prime}} \operatorname{card}\left(\frac{\Sigma_{\mathbf{q}}(S)+G^{\prime}(\overline{\mathbb{Q}})}{G^{\prime}(\overline{\mathbb{Q}})}\right) \mathscr{H}\left(G^{\prime} ; \widetilde{D}_{0}, \widetilde{D}_{1}, \ldots, \widetilde{D}_{n}\right)}{C_{0} \mathscr{H}\left(G_{0} \times G ; \widetilde{D}_{0}, \widetilde{D}_{1}, \ldots, \widetilde{D}_{n}\right)}\right)^{1 /\left(r^{\prime}-\lambda^{\prime}\right)}
$$

et on pose $B\left(G^{\prime}\right)=A\left(G^{\prime}\right)^{\left(r^{\prime}-\lambda^{\prime}\right) / r^{\prime}} \max \left\{1, A\left(G^{\prime}\right)\right\}^{\lambda^{\prime} / r^{\prime}}$.

Comme expliqué à la page 231 de [21], la quantité

$$
x:=\inf \left\{B\left(G^{\prime}\right) \mid t_{G^{\prime}}+W \neq t_{G_{0} \times G}\right\},
$$

où $G^{\prime}$ varie parmi les sous-groupes algébriques connexes $G^{\prime} \operatorname{de} G_{0} \times_{\overline{\mathbb{Q}}} G$ tel que $t_{G^{\prime}}+W \neq$ $t_{G_{0} \times G}$, est un nombre réel strictement positif. En effet, si $B\left(G^{\prime}\right) \leq B(\{0\})$, alors la quantité $\mathscr{H}\left(G^{\prime} ; \widetilde{D}_{0}, \widetilde{D}_{1}, \ldots, \widetilde{D}_{n}\right)$ est bornée, et par conséquent le degré $\operatorname{deg} G^{\prime}$ est borné. 
Puisque les coefficients de $\mathscr{H}\left(G^{\prime} ; X_{0}, X_{1}, \ldots, X_{n}\right)$ sont des entiers naturels inférieurs à $\operatorname{deg} G^{\prime}$, on en déduit que $\mathscr{H}\left(G^{\prime} ; \widetilde{D}_{0}, \widetilde{D}_{1}, \ldots, \widetilde{D}_{n}\right)$ appartient à un ensemble fini (à $\widetilde{D}_{0}, \widetilde{D}_{1}, \ldots, \widetilde{D}_{n}$ fixés). Par conséquent l'ensemble

$$
\left\{B\left(G^{\prime}\right) \mid B\left(G^{\prime}\right) \leq B(\{0\}), t_{G^{\prime}}+W \neq t_{G_{0} \times G}\right\}
$$

est fini, d'où

$$
x=\min \left\{B\left(G^{\prime}\right) \mid t_{G^{\prime}}+W \neq t_{G_{0} \times G}\right\}>0 .
$$

Fixons un sous-groupe algébrique connexe $\widetilde{G}$ de $G_{0} \times_{\overline{\mathbb{Q}}} G$ tel que $B(\widetilde{G})=x$. Pour tout $i \in\{0, \ldots, n\}$, on pose également $D_{i}^{\#}=x \widetilde{D}_{i}, D_{i}=\left[D_{i}^{\#}\right]$ et $D_{i}^{\prime}=\max \left\{1, D_{i}\right\}$. Le résultat suivant est le lemme 3.3 de [21], et découle facilement des définitions précédentes.

Lemme 8.2. Supposons que $x \leq 1$. Alors pour tout sous-groupe algébrique connexe $G^{\prime}$ de $G_{0} \times_{\overline{\mathbb{Q}}} G$ tel que $t_{G^{\prime}}+W \neq t_{G_{0} \times G}$, l'inégalité suivante est vérifiée :

$\widetilde{T}^{\lambda^{\prime}} \operatorname{card}\left(\frac{\Sigma_{\mathbf{q}}(S)+G^{\prime}(\overline{\mathbb{Q}})}{G^{\prime}(\overline{\mathbb{Q}})}\right) \mathscr{H}\left(G^{\prime} ; D_{0}^{\#}, D_{1}^{\#}, \ldots, D_{n}^{\#}\right) \geq C_{0} \mathscr{H}\left(G_{0} \times G ; D_{0}^{\#}, D_{1}^{\#}, \ldots, D_{n}^{\#}\right)$.

De plus, cette inégalité est une égalité pour $G^{\prime}=\widetilde{G}$.

Démonstration. Nous reprenons les arguments de [21. Posons

$$
\mho_{G^{\prime}}=\frac{\widetilde{T}^{\lambda^{\prime}} \operatorname{card}\left(\frac{\Sigma_{\mathbf{q}}(S)+G^{\prime}(\overline{\mathbb{Q}})}{G^{\prime}(\overline{\mathbb{Q}})}\right) \mathscr{H}\left(G^{\prime} ; D_{0}^{\#}, D_{1}^{\#}, \ldots, D_{n}^{\#}\right)}{C_{0} \mathscr{H}\left(G_{0} \times G ; D_{0}^{\#}, D_{1}^{\#}, \ldots, D_{n}^{\#}\right)} .
$$

Par homogénéité de $\mathscr{H}$, on a $x^{r^{\prime}} \mho_{G^{\prime}}=A\left(G^{\prime}\right)^{r^{\prime}-\lambda^{\prime}}$. Si $A\left(G^{\prime}\right) \geq 1$, alors $\mho_{G^{\prime}} \geq 1 / x^{r^{\prime}} \geq 1$. Si $A\left(G^{\prime}\right)<1$, alors par définition on a $B\left(G^{\prime}\right)=A\left(G^{\prime}\right)^{\left(r^{\prime}-\lambda^{\prime}\right) / r^{\prime}} \geq x$, puis $\mho_{G^{\prime}} \geq 1$. On a donc démontré l'inégalité 8.1). De plus comme $x \leq 1$ on a $x=A(\widetilde{G})^{(\widetilde{r}-\widetilde{\lambda}) / \widetilde{r}}$, d'où $\mho_{\widetilde{G}}=1$ (où $\widetilde{\lambda}=\operatorname{codim}_{W}\left(t_{\widetilde{G}} \cap W\right)$ et $\widetilde{r}=\operatorname{codim}_{G_{0} \times G} \widetilde{G}$ ).

Le résultat suivant est une conséquence du théorème 5.1 et du lemme 8.2 .

LEMme 8.3. Si la place $v_{0}$ est finie, alors

$$
\operatorname{card}\left(\frac{\Sigma_{\mathbf{q}}(S)+\widetilde{G}(\overline{\mathbb{Q}})}{\widetilde{G}(\overline{\mathbb{Q}})}\right)=S+1 .
$$

Supposons que $x \leq 1$ et que la place $v_{0}$ est archimédienne. Il existe une constante $q_{21} \geq 1$ telle que si

$$
d(u, V)<\frac{1}{C_{0} q_{21} S D_{0}^{\prime}\left(D_{1}^{\prime}\right)^{g_{1}} \cdots\left(D_{n}^{\prime}\right)^{g_{n}} \exp (\max \{1, h(W)\})},
$$

alors il n'existe pas d'entier $s \in\{1, \ldots, S\}$ tel que sq $\in \widetilde{G}(\overline{\mathbb{Q}})$. En particulier

$$
\operatorname{card}\left(\frac{\Sigma_{\mathbf{q}}(S)+\widetilde{G}(\overline{\mathbb{Q}})}{\widetilde{G}(\overline{\mathbb{Q}})}\right)=S+1
$$

Démonstration. Si la place $v_{0}$ est finie, l'exponentielle exp réalise un difféomorphisme de $D\left(0, r_{p_{0}}\right)$ sur son image. S'il existe $s \in \mathbb{N} \backslash\{0\}$ tel que $s \mathbf{q} \in \widetilde{G}(\overline{\mathbb{Q}})$, on en déduit que $(1, u) \in t_{\widetilde{G}}\left(\mathbb{C}_{v_{0}}\right)$, ce qui est absurde d'après l'hypothèse 1 (par construction, on a $t_{\widetilde{G}}+W \neq$ $\left.t_{G_{0} \times G}\right)$. Supposons que la place $v_{0}$ est archimédienne. Nous allons appliquer le théorème $5.1(1)$ à $\mathcal{G}=G_{0} \times G, \mathcal{H}=G_{0} \times H, \mathcal{G}^{\prime}=\widetilde{G}$ et au point $(1, u) \in t_{G_{0} \times G}\left(\mathbb{C}_{v_{0}}\right)$ (remarquons 
que $d(u, V)=d((1, u), W))$. L'hypothèse 1 correspond alors à celle du théorème 5.1 (1). Par définition de $\widetilde{G}$, on a $t_{\widetilde{G}}+W \neq t_{G_{0} \times G}$. De plus, d'après le lemme 8.2 , on a

$$
\widetilde{T}^{\widetilde{\lambda}} \operatorname{card}\left(\frac{\Sigma_{\mathbf{q}}(S)+\widetilde{G}(\overline{\mathbb{Q}})}{\widetilde{G}(\overline{\mathbb{Q}})}\right) \mathscr{H}\left(\widetilde{G} ; D_{0}^{\#}, D_{1}^{\#}, \ldots, D_{n}^{\#}\right)=C_{0} \mathscr{H}\left(G_{0} \times G ; D_{0}^{\#}, D_{1}^{\#}, \ldots, D_{n}^{\#}\right) \text {. }
$$

D'après [28, propriété 4.4], pour tout $i \in\{0, \ldots, n\}$, l'application partielle

$$
x_{i} \mapsto \mathscr{H}\left(G_{0} \times G ; x_{0}, \ldots, x_{n}\right) / \mathscr{H}\left(\widetilde{G} ; x_{0}, \ldots, x_{n}\right)
$$

est croissante. Comme $D_{i}^{\#} \leq 2 D_{i}^{\prime}$, on en déduit que

$$
\widetilde{T}^{\widetilde{\lambda}} \operatorname{card}\left(\frac{\Sigma_{\mathbf{q}}(S)+\widetilde{G}(\overline{\mathbb{Q}})}{\widetilde{G}(\overline{\mathbb{Q}})}\right) \mathscr{H}\left(\widetilde{G} ; D_{0}^{\prime}, D_{1}^{\prime}, \ldots, D_{n}^{\prime}\right) \leq C_{0} 2^{g+1} \mathscr{H}\left(G_{0} \times G ; D_{0}^{\prime}, D_{1}^{\prime}, \ldots, D_{n}^{\prime}\right) .
$$

Comme $1 \leq D_{i}^{\prime}$ pour tout $i \in\{0,1, \ldots, n\}$, on a par ailleurs

$$
\operatorname{deg} \widetilde{G}=\mathscr{H}(\widetilde{G} ; 1, \ldots, 1) \leq \mathscr{H}\left(\widetilde{G} ; D_{0}^{\prime}, D_{1}^{\prime}, \ldots, D_{n}^{\prime}\right),
$$

et donc

$$
\begin{aligned}
\operatorname{deg} \widetilde{G} & \leq C_{0} 2^{g+1} \mathscr{H}\left(G_{0} \times G ; D_{0}^{\prime}, D_{1}^{\prime}, \ldots, D_{n}^{\prime}\right) \\
& =C_{0} 2^{g+1} \operatorname{deg}\left(G_{0} \times G\right) D_{0}^{\prime}\left(D_{1}^{\prime}\right)^{g_{1}} \cdots\left(D_{n}^{\prime}\right)^{g_{n}} .
\end{aligned}
$$

On conclut alors avec le théorème 5.1 (1)

8.2. Fibré adélique hermitien des sections auxiliaires. Soit $\left(P_{\lambda_{0}}\right)_{0 \leq \lambda_{0} \leq D_{0}}$ une base de l'espace vectoriel $K[X]_{\leq D_{0}}$ des polynômes de degré inférieur ou égal à $D_{0}$ avec $P_{0}=1$. Notons $N_{0}=1$ et $\mathbb{P}=\mathbb{P}_{K}^{N_{0}} \times \cdots \times \mathbb{P}_{K}^{N_{n}}$. Pour tout $i \in\{0, \ldots, n\}$, on fixe des coordonnées homogènes $\left(X_{i, j}\right)_{0 \leq j \leq N_{i}}$ de $\mathbb{P}_{K}^{N_{i}}$. Notons également $\mathbf{D}=\left(D_{0}, \ldots, D_{n}\right)$ et $K[\mathbb{P}]$ la $K$ algèbre multigraduée des polynômes multihomogènes en les variables $\left(X_{i, j}\right)_{0 \leq i \leq n, 0 \leq j \leq N_{i}}$. Soit $E$ l'espace des polynômes de multidegré $\mathbf{D}$ qui ne s'annulent pas identiquement sur $G_{0} \times G$; en notant $I_{G_{0} \times G}$ l'idéal annulateur de $G_{0} \times G$ dans $\mathbb{P}$, on a ainsi $E=$ $\left(K[\mathbb{P}] / I_{G_{0} \times G}\right)_{\mathbf{D}}$. Considérons l'ensemble

$$
\Lambda=\left\{\boldsymbol{\lambda}=\left(\lambda_{0},\left(\boldsymbol{\lambda}_{i}\right)_{1 \leq i \leq n}\right) \in \mathbb{N} \times \prod_{i=1}^{n} \mathbb{N}^{N_{i}+1}\left|\lambda_{0} \leq D_{0}, \boldsymbol{\lambda}_{i}=\left(\lambda_{i, j}\right)_{0 \leq j \leq N_{i}},\right| \boldsymbol{\lambda}_{i} \mid=D_{i}\right\} .
$$

L'ensemble des classes de polynômes de la forme

$$
X_{0,0}^{\operatorname{deg} P_{\lambda_{0}}} P_{\lambda_{0}}\left(\frac{X_{0,1}}{X_{0,0}}\right) \prod_{i=1}^{n} \prod_{j=0}^{N_{i}} X_{i, j}^{\lambda_{i, j}}, \quad \lambda \in \Lambda,
$$

est une famille génératrice de $E$. Considérons une famille de tels polynômes dont les classes $s_{1}, \ldots, s_{\operatorname{dim} E}$ forment une base de $E$. On définit alors un fibré adélique hermitien $\left(E,\left(\|\cdot\|_{E, v}\right)_{v}\right)$ en choisissant pour chaque place $v \in \Sigma_{K}$ la norme $\|\cdot\|_{E, v}$ rendant cette base orthonormée. On a ainsi $\widehat{\mu}_{\mathrm{n}}(E)=0$. Concrètement, un élément $s$ de $E$ s'écrit comme la classe d'équivalence d'un polynôme

$$
P=\sum_{\lambda \in \Lambda} p_{\boldsymbol{\lambda}} X_{0,0}^{\operatorname{deg} P_{\lambda_{0}}} P_{\lambda_{0}}\left(\frac{X_{0,1}}{X_{0,0}}\right) \prod_{i=1}^{n} \prod_{j=0}^{N_{i}} X_{i, j}^{\lambda_{i, j}}
$$


On note alors

$$
F_{s}=P \circ\left(\Psi_{0} \times \Psi\right): \mathbb{C}_{v_{0}} \times \mathscr{U} \rightarrow \mathbb{C}_{v_{0}},
$$

avec $\Psi_{0}(z)=(1, z)$ pour tout $z \in \mathbb{C}_{v_{0}}$. Cette application est bien définie car elle ne dépend pas du choix d'un polynôme $P$ représentant $s$. Pour toute section $s \in E$, il existe donc une unique famille $\left(p_{\boldsymbol{\lambda}}\right)_{\boldsymbol{\lambda} \in \Lambda}$ d'éléments de $K$ telle que pour tout $\mathbf{z}=\left(z_{0}, \mathbf{z}_{1}, \ldots, \mathbf{z}_{n}\right) \in \mathbb{C}_{v_{0}} \times \mathscr{U}$, on ait

$$
F_{s}(\mathbf{z})=\sum_{\boldsymbol{\lambda} \in \Lambda} p_{\boldsymbol{\lambda}} P_{\lambda_{0}}\left(z_{0}\right) \prod_{i=1}^{n} \prod_{j=0}^{N_{i}} \varphi_{i, j}\left(\mathbf{z}_{i}\right)^{\lambda_{i, j}} .
$$

Avec ces notations, pour toute place $v$ de $K$, la norme $\|\cdot\|_{E, v}$ satisfait

$$
\|s\|_{E, v}=\left|\left(p_{\boldsymbol{\lambda}}\right)_{\boldsymbol{\lambda} \in \Lambda}\right|_{v}= \begin{cases}\left(\sum_{\boldsymbol{\lambda} \in \Lambda}\left|p_{\boldsymbol{\lambda}}\right|_{v}^{2}\right)^{1 / 2} & \text { si } v \text { est archimédienne, } \\ \max _{\boldsymbol{\lambda} \in \Lambda}\left|p_{\boldsymbol{\lambda}}\right|_{v} & \text { sinon. }\end{cases}
$$

Nous allons maintenant définir une norme particulière en la place $v_{0}$. Rappelons que $S_{0}$ et $T=[\widetilde{T}]$ sont des entiers strictement positifs. On considère l'ensemble

$$
\mathbf{\Upsilon}=\left\{(m, \boldsymbol{\tau}) \in \mathbb{N} \times \mathbb{N}^{g+1-t}\left|m \leq S_{0},\right| \boldsymbol{\tau} \mid \leq 2(g+1) T\right\} .
$$

Soit $\left(w_{1}, \ldots, w_{g-t}\right)$ une base de $V \otimes_{K} \mathbb{C}_{v_{0}}$ formée de vecteurs de norme égale à 1 . On considère alors la base de $W \otimes_{K} \mathbb{C}_{v_{0}}$ donnée par $\mathbf{w}=\left(e_{0}, w_{1}, \ldots, w_{g-t}\right)$. Considérons la matrice $\mathcal{A}_{0}$ de taille card $\boldsymbol{\Upsilon} \times \operatorname{dim} E$ définie par : pour tout $(m, \boldsymbol{\tau}) \in \boldsymbol{\Upsilon}$ et pour tout $i \in\{1, \ldots, \operatorname{dim} E\}$,

$$
\mathcal{A}_{0}[(m, \boldsymbol{\tau}), i]=\frac{1}{\boldsymbol{\tau} !} D_{\mathbf{w}}^{\boldsymbol{\tau}} F_{s_{i}}(m, m u) .
$$

Étant donné un nombre réel $\alpha>0$ et une place $v$ de $K$, on définit la norme $\|\cdot\|_{\alpha, v}$ sur $E \otimes_{K} \mathbb{C}_{v}$ en posant $\|\cdot\|_{\alpha, v}=\|\cdot\|_{E, v}$ si $v \neq v_{0}$ et

$$
\|s\|_{\alpha, v_{0}}= \begin{cases}\max \left\{\|s\|_{E, v_{0}}, \alpha\left|\mathcal{A}_{0} s\right|_{v_{0}}\right\} & \text { si } v_{0} \text { est finie } \\ \left(\|s\|_{E, v_{0}}^{2}+\left(\alpha\left|\mathcal{A}_{0} s\right|_{v_{0}}\right)^{2}\right)^{1 / 2} & \text { sinon. }\end{cases}
$$

Remarquons que pour toute place $v \in \Sigma_{K}$, on a l'inégalité $\|\cdot\|_{E, v} \leq\|\cdot\|_{\alpha, v}$. On note $h_{\alpha}$ la hauteur (logarithmique et absolue) sur $E \otimes_{K} \overline{\mathbb{Q}}$ associée à cette famille de normes : pour toute extension finie $K^{\prime}$ de $K$ et pour toute section non nulle $s \in E \otimes_{K} K^{\prime}$, on a

$$
h_{\alpha}(s)=\sum_{v \in \Sigma_{K^{\prime}}} \frac{\left[K_{v}^{\prime}: \mathbb{Q}_{v}\right]}{\left[K^{\prime}: \mathbb{Q}\right]} \log \|s\|_{\alpha, v} .
$$

8.3. Lemme de multiplicités. L'objet de ce paragraphe est de démontrer le résultat suivant, qui repose sur un lemme de multiplicités dû à Philippon [32, 33].

LEMME 8.4. Supposons que $x \leq 1$. Il existe une constante $\overline{222} \geq 1$ vérifiant la propriété suivante. Si les conditions

(a) $\left(\varlimsup_{22} S D_{0}^{\prime}\left(D_{1}^{\prime}\right)^{g_{1}} \cdots\left(D_{n}^{\prime}\right)^{g_{n}}\right)^{-1}>d(u, V)$;

(b) $\min \{T, S\}>C_{0}>q_{22}$;

(c) $T>\underset{22}{2} \max \left\{D_{0}^{\prime} /(S+1), D_{1}^{\prime}, \ldots, D_{n}^{\prime}\right\}$

sont vérifiées, alors il n'existe pas d'élément $s \in E \otimes_{K} \overline{\mathbb{Q}} \backslash\{0\}$ tel que

$$
\mathcal{D}_{\mathbf{w}}^{\boldsymbol{\tau}} F_{s}(m, m u)=0 \quad \forall(m, \boldsymbol{\tau}) \in \mathbb{N} \times \mathbb{N}^{g+1-t}, m \leq(g+1) S,|\boldsymbol{\tau}| \leq(g+1) T .
$$


Démonstration. Nous raisonnons par l'absurde en supposant qu'il existe une solution $s \in E \otimes_{K} \overline{\mathbb{Q}} \backslash\{0\}$ au système d'égalités 8.3 . D'après le lemme de multiplicités de Philippon [33], il existe un sous-groupe algébrique connexe $G^{\prime} \subsetneq G_{0} \times_{\overline{\mathbb{Q}}} G$ tel que

$$
T^{\lambda^{\prime}} \operatorname{card}\left(\frac{\Sigma_{\mathbf{q}}(S)+G^{\prime}(\overline{\mathbb{Q}})}{G^{\prime}(\overline{\mathbb{Q}})}\right) \mathscr{H}\left(G^{\prime} ; D_{0}^{\prime}, D_{1}^{\prime}, \ldots, D_{n}^{\prime}\right) \leq 2^{g+1} \mathscr{H}\left(G_{0} \times G ; D_{0}^{\prime}, D_{1}^{\prime}, \ldots, D_{n}^{\prime}\right) .
$$

Étape 1: Montrons que $t_{G^{\prime}}+W \neq t_{G_{0} \times G}$. Par l'absurde, si $t_{G^{\prime}}+W=t_{G_{0} \times G}$ alors $\lambda^{\prime}=r^{\prime}=\operatorname{codim}_{G_{0} \times G} G^{\prime}$. Comme $G$ est une variété semi-abélienne, le groupe $G^{\prime}$ s'écrit $G^{\prime}=G_{0}^{\prime} \times A$, où $G_{0}^{\prime}$ est un sous-groupe de $G_{0}$ et $A$ est un sous-groupe de $G$. Si $G_{0}^{\prime}$ est égal à $G_{0}$, alors l'inégalité (8.4) entraîne

$$
T^{r^{\prime}} \leq 2^{g+1} \operatorname{deg}\left(G_{0} \times G\right) \max \left\{D_{1}^{\prime}, \ldots, D_{n}^{\prime}\right\}^{r^{\prime}} .
$$

Si $G_{0}^{\prime}=\{0\}$, alors pour tout $m \in \mathbb{N} \backslash\{0\}$, on a $m \mathbf{q}=(m, m \mathbf{p}) \notin G^{\prime}=\{0\} \times A$, donc

$$
\operatorname{card}\left(\frac{\Sigma_{\mathbf{q}}(S)+G^{\prime}(\overline{\mathbb{Q}})}{G^{\prime}(\overline{\mathbb{Q}})}\right)=S+1
$$

D'après (8.4), on a alors

$$
T^{r^{\prime}} \leq 2^{g+1} \operatorname{deg}\left(G_{0} \times G\right) \frac{D_{0}^{\prime}}{S+1} \max \left\{D_{1}^{\prime}, \ldots, D_{n}^{\prime}\right\}^{r^{\prime}-1} .
$$

Dans tous les cas, l'hypothèse $(\mathrm{c})$ du lemme est contredite pourvu que la constante $q_{22}$ soit choisie suffisamment grande. On a donc bien $t_{G^{\prime}}+W \neq t_{G_{0} \times G}$.

Étape 2: Montrons que $\lambda^{\prime} \geq 1$ ou bien que pour tout $m \in\{1, \ldots, S\}, m \mathbf{q} \notin G^{\prime}(K)$. D'après la première étape, $t_{G^{\prime}}+W \neq t_{G_{0} \times G}$, donc d'après l'hypothèse 1 on a $(1, u) \notin$ $t_{G^{\prime}}\left(\mathbb{C}_{v_{0}}\right)$. Si $\lambda^{\prime}=\operatorname{codim}_{W}\left(t_{G}^{\prime} \cap W\right)=0$, alors $W \subseteq t_{G}^{\prime}$. D'après l'inégalité (8.4), on a

$$
\operatorname{deg} G^{\prime} \leq 2^{g+1} \operatorname{deg}\left(G_{0} \times G\right) D_{0}^{\prime}\left(D_{1}^{\prime}\right)^{g_{1}} \cdots\left(D_{n}^{\prime}\right)^{g_{n}} .
$$

D'après la condition (a), on en déduit que

$$
d(u, V)=d((1, u), W) \leq \frac{2^{g+1} \operatorname{deg}\left(G_{0} \times G\right)}{q_{22} S \operatorname{deg} G^{\prime}} .
$$

En appliquant le second point du théorème 5.1 au vecteur $(1, u)$ avec $\mathcal{G}=G_{0} \times G, \mathcal{G}^{\prime}=G^{\prime}$, $\mathcal{H}=G_{0} \times H$, on en déduit que pour tout $0<m \leq S, m \mathbf{q} \notin G^{\prime}\left(\overline{\mathbb{Q}}\right.$ ) (pourvu que $q_{22}$ soit plus grande qu'une constante ne dépendant que de $\left.G, \phi,\|\cdot\|_{v_{0}}\right)$.

Étape 3 : Nous allons conclure en montrant que l'inégalité 8.4 contredit le lemme 8.2 . Premièrement, remarquons qu'il existe $i \in\{0, \ldots, n\}$ tel que $D_{i} \neq 0$. En effet, sinon tous les $D_{i}^{\prime}$ sont égaux à 1 et l'inégalité (8.4) entraîne

$$
T^{\lambda^{\prime}} \operatorname{card}\left(\frac{\Sigma_{\mathbf{q}}(S)+G^{\prime}(\overline{\mathbb{Q}})}{G^{\prime}(\overline{\mathbb{Q}})}\right) \leq 2^{g+1} \operatorname{deg}\left(G_{0} \times G\right) .
$$

D'après l'étape 2 , on obtient $\min \{T, S+1\} \leq 2^{g+1} \operatorname{deg}\left(G_{0} \times G\right)$, ce qui contredit la condition (b). Il existe donc au moins un entier $i \in\{0, \ldots, n\}$ tel que $D_{i} \neq 0$. On considère alors les entiers $0 \leq k_{1}<\cdots<k_{h} \leq n$ pour lesquels $D_{k_{i}} \neq 0,1 \leq i \leq h$, et on 
note $\pi$ la projection

$$
\pi: G_{0} \times G \rightarrow \prod_{i=1}^{h} G_{k_{i}}
$$

D'après les propriétés du polynôme $\mathscr{H}$ rappelées au paragraphe 1.2 (page 9), on a

$$
\begin{aligned}
\mathscr{H}\left(G_{0} \times G ; D_{0}^{\prime}, \ldots, D_{n}^{\prime}\right) & =(g+1) ! \prod_{D_{i}=0} \frac{\operatorname{deg} G_{i}}{g_{i} !} \times \prod_{D_{j} \neq 0} \frac{\mathscr{H}\left(G_{j}, D_{j}\right)}{g_{j} !} \\
& \leq \frac{\operatorname{deg}\left(G_{0} \times G\right)}{\left(\operatorname{dim} \pi\left(G_{0} \times G\right)\right) !}\left(\prod_{D_{i} \neq 0} g_{i} !\right) \cdot \mathscr{H}\left(\pi\left(G_{0} \times G\right) ; D_{k_{1}}, \ldots, D_{k_{h}}\right) \\
& \leq \operatorname{deg}\left(G_{0} \times G\right) \cdot \mathscr{H}\left(\pi\left(G_{0} \times G\right) ; D_{k_{1}}, \ldots, D_{k_{h}}\right)
\end{aligned}
$$

(remarquons que $\left(\operatorname{dim} \pi\left(G_{0} \times G\right)\right) ! \prod_{D_{i} \neq 0} \frac{1}{g_{i} !} \in \mathbb{N} \backslash\{0\}$ est un coefficient multinomial). On a aussi

$$
\mathscr{H}\left(\pi\left(G^{\prime}\right) ; D_{k_{1}}, \ldots, D_{k_{h}}\right) \leq \mathscr{H}\left(G^{\prime} ; D_{0}^{\prime}, \ldots, D_{n}^{\prime}\right) .
$$

En utilisant l'inégalité (8.4), on obtient

$$
T^{\lambda^{\prime}} \operatorname{card}\left(\frac{\Sigma_{\mathbf{q}}(S)+G^{\prime}(\overline{\mathbb{Q}})}{G^{\prime}(\overline{\mathbb{Q}})}\right) \leq 2^{g+1} \operatorname{deg}\left(G_{0} \times G\right) \cdot \frac{\mathscr{H}\left(\pi\left(G_{0} \times G\right) ; D_{k_{1}}, \ldots, D_{k_{h}}\right)}{\mathscr{H}\left(\pi\left(G^{\prime}\right) ; D_{k_{1}}, \ldots, D_{k_{h}}\right)} .
$$

Puisque $D_{i}=\left[D_{i}^{\#}\right] \leq D_{i}^{\#}$ pour tout $i \in\{0, \ldots, n\}$, on obtient l'inégalité

$$
T^{\lambda^{\prime}} \operatorname{card}\left(\frac{\Sigma_{\mathbf{q}}(S)+G^{\prime}(\overline{\mathbb{Q}})}{G^{\prime}(\overline{\mathbb{Q}})}\right) \leq 2^{g+1} \operatorname{deg}\left(G_{0} \times G\right) \cdot \frac{\mathscr{H}\left(\pi\left(G_{0} \times G\right) ; D_{k_{1}}^{\#}, \ldots, D_{k_{h}}^{\#}\right)}{\mathscr{H}\left(\pi\left(G^{\prime}\right) ; D_{k_{1}}^{\#}, \ldots, D_{k_{h}}^{\#}\right)}
$$

par croissance des applications partielles

$$
x_{i} \mapsto \mathscr{H}\left(\pi\left(G_{0} \times G\right) ; x_{k_{1}}, \ldots, x_{k_{h}}\right) / \mathscr{H}\left(\pi\left(G^{\prime}\right) ; x_{k_{1}}, \ldots, x_{k_{h}}\right)
$$

(voir [28, propriété 4.4]). On considère le groupe $G^{\prime \prime}$ (vu comme un sous-groupe de $G_{0} \times G$ après permutation éventuelle des facteurs) défini par

$$
G^{\prime \prime}=\pi\left(G^{\prime}\right) \times \prod_{j \notin\left\{k_{1}, \ldots, k_{h}\right\}} G_{j} .
$$

Le sous-groupe $G^{\prime \prime}$ est distinct de $G_{0} \times G$, car sinon $\pi\left(G^{\prime}\right)=\pi\left(G_{0} \times G\right)$ et l'inégalité (8.5) ainsi que l'étape 2 entraînent $\min \{T, S+1\} \leq 2^{g+1} \operatorname{deg}\left(G_{0} \times G\right)$, ce qui contredit la condition (b) pourvu que $q 22 \geq 2^{g+1} \operatorname{deg}\left(G_{0} \times G\right)$. Par ailleurs, on a

$$
\frac{\mathscr{H}\left(\pi\left(G_{0} \times G\right) ; D_{k_{1}}^{\#}, \ldots, D_{k_{h}}^{\#}\right)}{\mathscr{H}\left(\pi\left(G^{\prime}\right) ; D_{k_{1}}^{\#}, \ldots, D_{k_{h}}^{\#}\right)}=\frac{\left(\operatorname{dim} G^{\prime \prime}\right) !\left(\operatorname{dim} \pi\left(G_{0} \times G\right)\right) !}{(g+1) !\left(\operatorname{dim} \pi\left(G^{\prime}\right)\right) !} \cdot \frac{\mathscr{H}\left(G_{0} \times G ; D_{0}^{\#}, \ldots, D_{n}^{\#}\right)}{\mathscr{H}\left(G^{\prime \prime} ; D_{0}^{\#}, \ldots, D_{n}^{\#}\right)}
$$

et

- $\operatorname{dim} \pi\left(G_{0} \times G\right)-\operatorname{dim} \pi\left(G^{\prime}\right)=\operatorname{codim}_{G_{0} \times G} G^{\prime \prime}=r^{\prime \prime}$,

- $\lambda^{\prime \prime}=\operatorname{codim}_{W}\left(W \cap t_{G^{\prime \prime}}\right) \leq \operatorname{codim}_{W}\left(W \cap t_{G^{\prime}}\right)=\lambda^{\prime}\left(\operatorname{car} G^{\prime} \subseteq G^{\prime \prime}\right)$,

- $\operatorname{card}\left(\frac{\Sigma_{\mathbf{q}}(S)+G^{\prime \prime}(\overline{\mathbb{Q}})}{G^{\prime \prime}(\overline{\mathbb{Q}})}\right) \leq \operatorname{card}\left(\frac{\Sigma_{\mathbf{q}}(S)+G^{\prime}(\overline{\mathbb{Q}})}{G^{\prime}(\overline{\mathbb{Q}})}\right)$. 
D'après 8.5), on en déduit qu'il existe une constante $c$ ne dépendant que de $g$ telle que

$$
T^{\lambda^{\prime \prime}} \operatorname{card}\left(\frac{\Sigma_{\mathbf{q}}(S)+G^{\prime \prime}(\overline{\mathbb{Q}})}{G^{\prime \prime}(\overline{\mathbb{Q}})}\right) \mathscr{H}\left(G^{\prime \prime} ; D_{0}^{\#}, \ldots, D_{n}^{\#}\right) \leq c \operatorname{deg} G \cdot \mathscr{H}\left(G_{0} \times G ; D_{0}^{\#}, \ldots, D_{n}^{\#}\right) .
$$

Pour tout $i \in\{0, \ldots, n\}$, on a par ailleurs $D_{i}^{\#} \leq\left[D_{i}^{\#}\right]+1=D_{i}+1 \leq 2 \max \left\{1, D_{i}\right\}=2 D_{i}^{\prime}$. D'après l'hypothèse (c), on a donc

$$
T \geq \frac{\underline{22}}{2} \max \left\{D_{0}^{\#} /(S+1), D_{1}^{\#}, \ldots, D_{n}^{\#}\right\} .
$$

Si $C_{0}>c \operatorname{deg}\left(G_{0} \times G\right) \operatorname{deg} G$, on montre que $t_{G^{\prime \prime}}+W \neq t_{G_{0} \times G}$ en utilisant les mêmes arguments qu'à l'étape 1 . On en déduit que si la constante q22 est suffisamment grande, l'inégalité (8.6) contredit le lemme 8.2 ce qui achève la démonstration.

8.4. Choix des paramètres. Nous allons maintenant fixer tous les paramètres introduits précédemment, à l'exception de la famille de polynômes $\left(P_{\lambda_{0}}\right)_{\lambda_{0} \leq D_{0}}$, que nous choisirons au paragraphe 8.10. Rappelons que l'on a noté $D=[K: \mathbb{Q}] /\left[K_{v_{0}}: \mathbb{Q}_{v_{0}}\right]$. Dans toute la suite, $C_{0}$ désigne un nombre réel supérieur à $\max \left\{q_{21}, q_{22}\right\} \geq 1$. Si $v_{0} \mid p_{0}$ est ultramétrique, on suppose également que $C_{0} \geq e^{2}$.

8.4.1. Cas archimédien. Supposons que la place $v_{0}$ est archimédienne. Soit $\mathfrak{e} \geq e$ un nombre réel et soit $\mathfrak{a} \geq 1$ un nombre réel tel que

$$
\mathfrak{a} \geq \frac{\max \left\{D, D h(W), \log \|u\|_{v_{0}}\right\}}{\log \mathfrak{e}}
$$

Posons alors $S_{0}:=\left[C_{0} \mathfrak{a}\right]$ et $S:=\left[C_{0}^{3} \mathfrak{a}\right]$. On définit également les quantités

$$
\begin{aligned}
A_{i} & :=D \max _{k \leq(g+1) S} h\left(k \mathbf{p}_{i}\right)+\left(1+C_{0}^{3} \mathfrak{a} \mathfrak{e}\left\|u_{i}\right\|_{v_{0}}\right)^{\rho_{i}}, \quad i \in\{1, \ldots, n\}, \\
A_{0} & :=D \log \left(e+\frac{C_{0}^{3} D}{\log \mathfrak{e}}\right)+\log \left(1+\frac{\mathfrak{e} C_{0}^{3} D}{\log \mathfrak{e}}\right), \\
U & :=C_{0} S_{0} \log (\mathfrak{e})\left(\frac{S+1}{C_{0} S_{0}}\right)^{1 / t}\left(\frac{C_{0} A_{0}}{\log \mathfrak{e}}+C_{0}^{2}\right)^{1 / t} \prod_{i=1}^{n}\left(C_{0}^{2}+\frac{C_{0} A_{i}}{S_{0} \log \mathfrak{e}}\right)^{g_{i} / t} .
\end{aligned}
$$

On pose alors

$$
\begin{aligned}
\widetilde{T} & :=\frac{U}{S_{0} \log \mathfrak{e}}, \\
\widetilde{D}_{i} & :=\frac{U}{C_{0} A_{i}+C_{0}^{2} S_{0} \log \mathfrak{e}}, \quad i \in\{1, \ldots, n\}, \\
\widetilde{D}_{0} & :=\frac{U}{C_{0} A_{0}+C_{0}^{2} \log \mathfrak{e}} .
\end{aligned}
$$

Rappelons que l'on a noté $T=[\widetilde{T}], D_{i}=\left[x \widetilde{D}_{i}\right]$ et $D_{i}^{\prime}=\max \left\{1, D_{i}\right\}$ pour tout $i \in$ $\{0, \ldots, n\}$. Les propriétés suivantes découlent facilement des définitions précédentes et seront utilisées de façon récurrente dans la suite.

LEMME 8.5. Les inégalités suivantes sont vérifiées :

(1) $x \leq 1$

(2) $\widetilde{T}>C_{0}^{2}, T>C_{0}$; 
(3) $\left(S_{0}+1\right) /(S+1) \leq 2 / C_{0}^{2}, S / S_{0} \leq 2 C_{0}^{2}$;

(4) $T>C_{0} \max \left\{D_{0}^{\prime} /(S+1), D_{1}^{\prime}, \ldots, D_{n}^{\prime}\right\}$;

(5) $D_{i} \leq D_{i}^{\prime} \leq \widetilde{D}_{i} \leq \widetilde{D}_{i} A_{i} \leq U / C_{0} \forall i \in\{0, \ldots, n\}$;

(6) $\widetilde{D}_{i} \max _{k \leq(g+1) S} h\left(k \mathbf{p}_{i}\right) \leq U /\left(D C_{0}\right) \forall i \in\{1, \ldots, n\}$;

(7) $\widetilde{D}_{i}\left(1+S \mathfrak{e}\left\|u_{i}\right\|_{v_{0}}\right)^{\rho_{i}} \leq U / C_{0} \forall i \in\{1, \ldots, n\}$.

Démonstration. Montrons le premier point. Par définition de $x$, on a l'implication

$$
\frac{1}{\operatorname{deg}\left(G_{0} \times G\right)} \frac{\widetilde{T}^{g+1-t}(S+1)}{C_{0} \widetilde{D}_{0} \widetilde{D}_{1}^{g_{1}} \cdots \widetilde{D}_{n}^{g_{n}}}=A(\{0\})^{t} \leq 1 \Longrightarrow x \leq 1 .
$$

La définition de $U$ correspond précisément à $A(\{0\})^{t}=\left(C_{0}^{t} \operatorname{deg}\left(G_{0} \times G\right)\right)^{-1} \leq 1$, ce qui démontre (1), Les points suivants sont des conséquences immédiates des définitions et de l'inégalité $x \leq 1$.

8.4.2. Cas ultramétrique. Supposons que la place $v_{0}$ est ultramétrique. Soit $\mathfrak{R}$ un nombre réel dans l'intervalle $] 1, r_{p_{0}} /\|u\|_{v_{0}}$ [ et soit $\mathfrak{a} \geq 1$ tel que

$$
\mathfrak{a} \log \mathfrak{R} \geq D \max \{1, h(W)\}+\log _{+}\left((\log \mathfrak{R})^{-1}\right) .
$$

Posons $S_{0}:=\left[C_{0} \mathfrak{a}\right]$ et $S:=\left[C_{0}^{3} \mathfrak{a}\right]$. On définit également

$$
\begin{aligned}
A_{i} & :=D \max _{k \leq(g+1) S} h\left(k \mathbf{p}_{i}\right), \quad i \in\{1, \ldots, n\}, \\
A_{0} & :=D \log \left(e+\frac{C_{0}^{3} D}{\log \mathfrak{R}}\right)+\log \left(1+\frac{\mathfrak{R} C_{0} D}{\log \mathfrak{R}}\right), \\
U & :=C_{0} S_{0} \log \mathfrak{R}\left(\frac{S+1}{C_{0} S_{0}}\right)^{1 / t}\left(\frac{C_{0} A_{0}}{\log \mathfrak{R}}+C_{0}^{2}\right)^{1 / t} \prod_{i=1}^{n}\left(C_{0}^{2}+\frac{C_{0} A_{i}}{S_{0} \log \mathfrak{R}}\right)^{g_{i} / t} .
\end{aligned}
$$

On pose alors

$$
\begin{aligned}
\widetilde{T} & :=\frac{U}{S_{0} \log \Re}, \\
\widetilde{D}_{i} & :=\frac{U}{C_{0} A_{i}+C_{0}^{2} S_{0} \log \Re}, \quad i \in\{1, \ldots, n\}, \\
\widetilde{D}_{0} & :=\frac{U}{C_{0} A_{0}+C_{0}^{2} \log \mathfrak{R}} .
\end{aligned}
$$

Rappelons que l'on a noté $T=[\widetilde{T}], D_{i}=\left[x \widetilde{D}_{i}\right]$ et $D_{i}^{\prime}=\max \left\{1, D_{i}\right\}$ pour tout $i \in$ $\{0, \ldots, n\}$. Comme dans le cas archimédien, énumérons quelques propriétés utiles satisfaites par ces paramètres.

LEMME 8.6. Les inégalités suivantes sont vérifiées :

(1) $x \leq 1$;

(2) $\widetilde{T}>C_{0}^{2}, T>C_{0}$;

(3) $\left(S_{0}+1\right) /(S+1) \leq 2 / C_{0}^{2}, S / S_{0} \leq 2 C_{0}^{2}$;

(4) $T>C_{0} \max \left\{D_{0}^{\prime} /(S+1), D_{1}^{\prime}, \ldots, D_{n}^{\prime}\right\}$;

(5) $D_{i} \leq D_{i}^{\prime} \leq \widetilde{D}_{i} \leq \widetilde{D}_{i} A_{i} \leq U / C_{0} \forall i \in\{0, \ldots, n\}$;

(6) $\widetilde{D}_{i} \max _{k \leq(g+1) S} h\left(k \mathbf{p}_{i}\right) \leq U /\left(D C_{0}\right) \forall i \in\{1, \ldots, n\}$;

(7) $S_{0} \log \mathfrak{R} \geq \log S_{0}$. 
Démonstration. Le premier point se démontre comme dans le cas archimédien, et les points (2) à (6) découlent immédiatement des définitions. Montrons (7). Si $\log \Re \geq$ $1 / \sqrt{C_{0}}$, on a

$$
S_{0} \log \mathfrak{R} \geq\left[C_{0} \mathfrak{a}\right] / \sqrt{C_{0}} \geq \sqrt{C_{0}} \mathfrak{a} \geq \sqrt{C_{0} \mathfrak{a}} \geq \log \left(C_{0} \mathfrak{a}\right) \geq \log S_{0}
$$

(l'avant-dernière inégalité découle de la croissance de $x \mapsto \sqrt{x} / \log x$ sur $\left[e^{2},+\infty[\right.$ ). Supposons que $\log \mathfrak{R}<1 / \sqrt{C_{0}}$. On doit montrer que $f\left(S_{0}\right) \geq 1 / \log \mathfrak{R}$, où $f(x)=x / \log x$ pour $x>1$. La fonction $f$ est croissante sur $\left[e,+\infty\left[\right.\right.$. Or $S_{0} \geq C_{0} \log (1 / \log \mathfrak{R}) / \log \mathfrak{R}$, et l'on en déduit que

$$
\begin{aligned}
f\left(S_{0}\right) & \geq \frac{C_{0} \log (1 / \log \mathfrak{R})}{\log \mathfrak{R}} \times \frac{1}{\log \log (1 / \log \mathfrak{R})+\log (1 / \log \mathfrak{R})+\log C_{0}} \\
& \geq \frac{C_{0} \log (1 / \log \mathfrak{R})}{\log \mathfrak{R}} \times \frac{1}{4 \log (1 / \log \mathfrak{R})}=\frac{C_{0}}{4 \log \mathfrak{R}} \geq \frac{1}{\log \mathfrak{R}} .
\end{aligned}
$$

8.4.3. Hypothèse centrale et organisation de la suite du texte. Nous allons raisonner par l'absurde afin de démontrer une minoration de la distance $d(u, V)$. Dans toute la suite, on fait l'hypothèse suivante.

Hypothèse 2. La distance $d(u, V)$ est inférieure à $\exp \left(-2 \sqrt{C_{0}} U\right)$.

En particulier, les conclusions des lemmes 8.3 et 8.4 sont satisfaites. Dans toute la suite du paragraphe 8 , on pose

$$
\alpha:=\exp \left(U\left(g+1+\sqrt{C_{0}}\right)\right) .
$$

Au paragraphe 8.5, nous allons majorer le rang et la norme d'opérateur de la matrice $\mathcal{A}_{0}$. Cela nous permettra d'appliquer le lemme 2.14 (page 15) pour construire une section non nulle $s \in E \otimes_{K} \overline{\mathbb{Q}}$ de «petite » hauteur $h_{\alpha}(s)$ (8.6). Nous construirons ensuite un jet de cette section. La suite de la preuve est consacrée à l'encadrement de la hauteur de ce jet, et aboutit à une contradiction. Nous en déduirons que l'hypothèse 2 est absurde, ce qui démontrera les théorèmes 4.3 et 4.4

8.5. Étude de la matrice $\mathcal{A}_{0}$. L'objet de ce paragraphe est de majorer le rang et la norme d'opérateur de la matrice $\mathcal{A}_{0}$.

8.5.1. Majoration du rang. Le rang $\operatorname{rg} \mathcal{A}_{0}$ de la matrice $\mathcal{A}_{0}$ est égal au rang du système

$$
\forall(m, \boldsymbol{\tau}) \in \mathbf{\Upsilon}, \quad D_{\mathbf{w}}^{\boldsymbol{\tau}} F_{s}(m, m u)=0
$$

en les inconnues $p_{i}$ définies par $s=\sum_{i=1}^{\operatorname{dim} E} p_{i} s_{i}$. On note $\widetilde{\lambda}=\operatorname{codim}_{W}\left(W \cap t_{\widetilde{G}}\right)$. Les arguments de la démonstration du lemme 6.7 de 35 . montrent que ce rang est inférieur à celui du système

$$
\forall(m, \boldsymbol{\tau}) \in \mathbb{N} \times \mathbb{N}^{\widetilde{\lambda}} ; 0 \leq m \leq S_{0},|\boldsymbol{\tau}| \leq 2(g+1) T, \quad D_{\mathbf{w}^{\prime}}^{\boldsymbol{\tau}} P(m \mathbf{q}+\widetilde{G})=0
$$

où $\mathbf{w}^{\prime}$ désigne une base d'un supplémentaire de $\left(W \otimes_{K} \overline{\mathbb{Q}}\right) \cap t_{\widetilde{G}}$ dans $W \otimes_{K} \overline{\mathbb{Q}}$ si $\tilde{\lambda} \geq 1$ (voir aussi la démonstration du lemme 6.1 de [14]), et où les inconnues du système sont les coordonnées des polynômes $P \in K[\mathbb{P}]_{\mathbf{D}}$ (dans une base quelconque de $K[\mathbb{P}]_{\mathbf{D}}$ ). On en déduit que

$$
\operatorname{rg} \mathcal{A}_{0} \leq \operatorname{card}\left\{\boldsymbol{\tau} \in \mathbb{N}^{\widetilde{\lambda}}|| \boldsymbol{\tau} \mid \leq 2(g+1) T\right\} \operatorname{card}\left(\frac{\Sigma_{\mathbf{q}}\left(S_{0}\right)+\widetilde{G}(\overline{\mathbb{Q}})}{\widetilde{G}(\overline{\mathbb{Q}})}\right) \operatorname{dim}\left(\overline{\mathbb{Q}}[\mathbb{P}] / I_{\widetilde{G}}\right)_{2 \mathbf{D}}
$$


où $I_{\widetilde{G}}$ désigne l'idéal des polynômes identiquement nuls sur $\widetilde{G}$. D'après un théorème de Chardin [11] (que l'on applique à un plongement de $\mathbb{P}$ dans un espace projectif, à la manière de [35, page 306]), il existe une constante $q_{23} \geq 1$ telle que

$$
\operatorname{dim}\left(\overline{\mathbb{Q}}[\mathbb{P}] / I_{\widetilde{G}}\right)_{2 \mathbf{D}} \leq q_{23} \mathscr{H}\left(\widetilde{G} ; D_{0}^{\prime}, \ldots, D_{n}^{\prime}\right) .
$$

Rappelons que pour tout $i \in\{0, \ldots, n\}$, l'application partielle

$$
x_{i} \mapsto \mathscr{H}\left(\widetilde{G} ; x_{0}, \ldots, x_{n}\right) / \mathscr{H}\left(G_{0} \times G ; x_{0}, \ldots, x_{n}\right)
$$

est décroissante (voir par exemple [28, propriété 4.4]). En remarquant que $D_{i}^{\#} \leq 2 D_{i}^{\prime}$ et en appliquant le lemme 8.2 , on obtient

$$
\begin{aligned}
\operatorname{rg} \mathcal{A}_{0} & \leq(2(g+1))^{g} q_{233} \widetilde{T}^{\widetilde{\lambda}} \operatorname{card}\left(\frac{\Sigma_{\mathbf{q}}\left(S_{0}\right)+\widetilde{G}(\overline{\mathbb{Q}})}{\widetilde{G}(\overline{\mathbb{Q}})}\right) \mathscr{H}\left(\widetilde{G} ; D_{0}^{\prime}, \ldots, D_{n}^{\prime}\right) \\
& \leq(2(g+1))^{g} 2^{g+1} C_{0} \frac{\operatorname{card}\left(\frac{\Sigma_{\mathbf{q}}\left(S_{0}\right)+\widetilde{G}(\overline{\mathbb{Q}})}{\widetilde{G}(\overline{\mathbb{Q}})}\right)}{\operatorname{card}\left(\frac{\Sigma_{\mathbf{q}}(S)+\widetilde{G}(\overline{\mathbb{Q}})}{\widetilde{G}(\overline{\mathbb{Q}})}\right)} \mathscr{H}\left(G_{0} \times G ; D_{0}^{\prime}, \ldots, D_{n}^{\prime}\right) .
\end{aligned}
$$

Par ailleurs, pour tout $i \in\{1, \ldots, n\}$, on a

$$
\lim _{\ell \rightarrow+\infty} \mathscr{H}\left(G_{i}, \ell\right) / \operatorname{dim}\left(K\left[\mathbb{P}^{N_{i}}\right] / I_{G_{i}}\right)_{\ell}=g_{i} !
$$

On en déduit qu'il existe une constante $q_{24}$ telle que pour tout $i \in\{1, \ldots, n\}$ et tout $\ell \in \mathbb{N}$,

$$
\mathscr{H}\left(G_{i}, \max \{1, \ell\}\right) / g_{i} ! \leq q_{24} \operatorname{dim}\left(K\left[\mathbb{P}^{N_{i}}\right] / I_{G_{i}}\right)_{\ell} .
$$

On a ainsi

$$
\begin{aligned}
\mathscr{H}\left(G_{0} \times G ; D_{0}^{\prime}, \ldots, D_{n}^{\prime}\right) & =(g+1) ! D_{0}^{\prime} \prod_{i=1}^{n} \frac{\mathscr{H}\left(G_{i}, D_{i}^{\prime}\right)}{g_{i} !} \\
& \leq q \frac{n}{24}(g+1) ! D_{0}^{\prime} \prod_{i=1}^{n} \operatorname{dim}\left(K\left[\mathbb{P}^{N_{i}}\right] / I_{G_{i}}\right)_{D_{i}}=q \frac{n}{24}(g+1) ! \operatorname{dim} E .
\end{aligned}
$$

D'après l'inégalité (8.7), on en déduit qu'il existe une constante $q_{25}$ telle que

$$
\frac{\operatorname{rg} \mathcal{A}_{0}}{\operatorname{dim} E} \leq q_{25} C_{0} \frac{\operatorname{card}\left(\frac{\Sigma_{\mathbf{q}}\left(S_{0}\right)+\widetilde{G}(\overline{\mathbb{Q}})}{\widetilde{G}(\overline{\mathbb{Q}})}\right)}{\operatorname{card}\left(\frac{\Sigma_{\mathbf{q}}(S)+\widetilde{G}(\overline{\mathbb{Q}})}{\widetilde{G}(\overline{\mathbb{Q}})}\right)} .
$$

En appliquant le lemme 8.3, on obtient la proposition suivante.

Proposition 8.7. Il existe une constante $q_{25}$ telle que

$$
\frac{\operatorname{rg} \mathcal{A}_{0}}{\operatorname{dim} E} \leq q_{25} C_{0} \frac{S_{0}+1}{S+1} \leq \frac{2 q_{25}}{C_{0}} .
$$

8.5.2. Majoration de la norme d'opérateur de $\mathcal{A}_{0}$. Nous allons maintenant majorer la quantité $\left\|\mathcal{A}_{0}\right\|_{v_{0}}$, définie par

$$
\left\|\mathcal{A}_{0}\right\|_{v_{0}}=\sup \left\{\left|\mathcal{A}_{0} s\right|_{v_{0}} /\|s\|_{E, v_{0}} \mid s \in\left(E \otimes_{K} \mathbb{C}_{v_{0}}\right) \backslash\{0\}\right\} .
$$

La majoration que nous allons démontrer repose sur les deux lemmes qui suivent. Soit $\left(v_{1}, \ldots, v_{g-t}\right)$ une base de $V \otimes_{K} \mathbb{C}_{v_{0}}$ telle que $\left\|v_{i}\right\|_{v_{0}} \leq 1$ pour tout $i \in\{1, \ldots, g-t\}$. On considère la base $\mathbf{v}$ de $W \otimes_{K} \mathbb{C}_{v_{0}}$ donnée par $\mathbf{v}=\left(e_{0}, v_{1}, \ldots, v_{g-t}\right)$. 
Lemme 8.8. Soit $\boldsymbol{\tau}=\left(\tau_{0}, \tau_{1}, \ldots, \tau_{g-t}\right) \in \mathbb{N}^{g+1-t}$ et soit $s \in E \otimes_{K} \mathbb{C}_{v_{0}}$. Si $\mathbf{e}^{\prime}=$ $\left(e_{0}, e_{1}^{\prime}, \ldots, e_{g}^{\prime}\right)$ est une base orthonormée de $t_{G_{0} \times G}\left(\mathbb{C}_{v_{0}}\right)$, alors pour tout $z \in \mathbb{C}_{v_{0}} \times \mathscr{U}$,

$$
\left|\frac{1}{\boldsymbol{\tau} !} \mathcal{D}_{\mathbf{v}}^{\boldsymbol{\tau}} F_{s}(z)\right|_{v_{0}} \leq q \frac{|\boldsymbol{\tau}|}{26} \max \left\{\left|\frac{1}{\boldsymbol{\sigma} !} \mathcal{D}_{\mathbf{e}^{\prime}}^{\boldsymbol{\sigma}} F_{s}(z)\right|_{v_{0}}\left|\boldsymbol{\sigma} \in \mathbb{N}^{g+1}, \sigma_{0}=\tau_{0},\right| \boldsymbol{\sigma}|=| \boldsymbol{\tau} \mid\right\},
$$

où $\overline{q_{26}}=g^{2}-1$ si $v_{0}$ est archimédienne et $\overline{q_{26}}=1$ sinon.

Démonstration. La base $\mathbf{e}^{\prime}$ identifie l'espace $t_{G_{0} \times G}\left(\mathbb{C}_{v_{0}}\right)$ à $\mathbb{C}_{v_{0}}^{g+1}$. Pour tout $i \in\{1, \ldots$, $g-t\}$, on note $\left(x_{i, j}\right)_{1 \leq j \leq g}$ les coordonnées de $v_{i}$ dans la base $\mathbf{e}^{\prime}$ :

$$
v_{i}=\sum_{j=1}^{g} x_{i, j} e_{j}^{\prime}
$$

Notons également

$$
\mathcal{T}=\left\{\boldsymbol{\sigma}=\left(\sigma_{i, j}\right)_{1 \leq i \leq g-t, 1 \leq j \leq g} \in \mathbb{N}^{g(g-t)}\left|\forall 1 \leq i \leq g-t, \sigma_{i, 1}+\cdots+\sigma_{i, g}=\right| \boldsymbol{\tau}_{i} \mid\right\} .
$$

On a ainsi

$$
\begin{aligned}
\frac{1}{\tau !} \mathcal{D}_{\mathbf{v}}^{\boldsymbol{\tau}} F_{s}(z)= & \left(\frac{1}{\tau_{0} !}\left(\frac{\partial}{\partial z_{0}}\right)^{\tau_{0}}\left(\prod_{i=1}^{g-t} \frac{1}{\tau_{i} !}\left(\sum_{j=1}^{g} x_{i, j} \frac{\partial}{\partial z_{j}}\right)^{\tau_{i}}\right) F_{s}\right)(z) \\
= & \sum_{\boldsymbol{\sigma} \in \mathcal{T}}\left(\prod_{\substack{1 \leq i \leq g-t \\
1 \leq j \leq g}} \frac{x_{i, j}^{\sigma_{i, j}}}{\sigma_{i, j} !}\right)\left(\frac{1}{\tau_{0} !}\left(\frac{\partial}{\partial z_{0}}\right)^{\tau_{0}}\right) \\
& \times\left(\prod_{k=1}^{g}\left(\frac{\partial}{\partial z_{k}}\right)^{\sigma_{1, k}+\cdots+\sigma_{g-t, k}}\right) F_{s}(z) \\
= & \sum_{\boldsymbol{\sigma} \in \mathcal{T}}\left(\prod_{i, j} x_{i, j}^{\sigma_{i, j}}\right) \times\left(\prod_{k=1}^{g} \frac{\left(\sigma_{1, k}+\cdots+\sigma_{g-t, k}\right) !}{\sigma_{1, k} ! \cdots \sigma_{g-t, k} !}\right) \\
& \times \frac{1}{\tau_{0} !}\left(\frac{\partial}{\partial z_{0}}\right)^{\tau_{0}} \times \prod_{\ell=1}^{g} \frac{1}{\left(\sigma_{1, \ell}+\cdots+\sigma_{g-t, \ell}\right) !}\left(\frac{\partial}{\partial z_{\ell}}\right)^{\sigma_{1, \ell}+\cdots+\sigma_{g-t, \ell}} F_{s}(z) .
\end{aligned}
$$

Par hypothèse, $\left|x_{i, j}\right|_{v_{0}} \leq 1$ pour tous $i, j$. Pour tout $k \in\{1, \ldots, g-t\}$, le coefficient multinomial

$$
\frac{\left(\sigma_{1, k}+\cdots+\sigma_{g-t, k}\right) !}{\sigma_{1, k} ! \cdots \sigma_{g-t, k} !}
$$

est un entier naturel, ce qui démontre le lemme si la place $v_{0}$ est finie. Dans le cas archimédien, il suffit de remarquer que

$$
\sum_{\boldsymbol{\sigma} \in \mathcal{T}} \prod_{k=1}^{g} \frac{\left(\sigma_{1, k}+\cdots+\sigma_{g-t, k}\right) !}{\sigma_{1, k} ! \cdots \sigma_{g-t, k} !} \leq((g-t)(g+1))^{|\boldsymbol{\tau}|}
$$

Lemme 8.9. Soit $\boldsymbol{\tau}=\left(\tau_{0}, \tau_{1}, \ldots, \tau_{g-t}\right) \in \mathbb{N}^{g+1-t}$ et soit $s \in E \otimes_{K} \mathbb{C}_{v_{0}}$. Si v $v_{0}$ est archimédienne, alors pour tout $z \in \mathbb{C}_{v_{0}} \times \mathbb{C}_{v_{0}}^{g_{1}} \times \cdots \times \mathbb{C}_{v_{0}}^{g_{n}} \simeq t_{G_{0} \times G}\left(\mathbb{C}_{v_{0}}\right)$, on a

$$
\left|\frac{1}{\boldsymbol{\tau} !} \mathcal{D}_{\mathbf{v}}^{\boldsymbol{\tau}} F_{s}(z)\right|_{v_{0}} \leq\left(g^{2}-1\right)^{|\boldsymbol{\tau}|} D_{0}^{\prime} \prod_{i=1}^{n} \exp \left(q_{27} D_{i}^{\prime}\left(1+\left\|z_{i}\right\|_{v_{0}}\right)^{\rho_{i}}\right) \max _{\lambda_{0} \leq D_{0}}\left|\frac{P_{\lambda_{0}}^{\left(\tau_{0}\right)}}{\tau_{0} !}\left(z_{0}\right)\right|_{v_{0}}\|s\|_{E, v_{0}}
$$


où $z_{i}$ désigne la projection de $z$ sur $\mathbb{C}_{v_{0}}^{g_{i}}$ et $q_{27} \geq 1$ est une constante. Si $v_{0}$ est une place finie, alors pour tout $z \in \mathbb{C}_{v_{0}} \times D\left(0, r_{p_{0}}\right)$,

$$
\left|\frac{1}{\boldsymbol{\tau} !} \mathcal{D}_{\mathbf{v}}^{\boldsymbol{\tau}} F_{s}(z)\right|_{v_{0}} \leq \max _{\lambda_{0} \leq D_{0}}\left|\frac{P_{\lambda_{0}}^{\left(\tau_{0}\right)}}{\tau_{0} !}\left(z_{0}\right)\right|_{v_{0}}\|s\|_{E, v_{0}} .
$$

Démonstration. On considère l'ensemble

$$
\Lambda=\left\{\boldsymbol{\lambda}=\left(\boldsymbol{\lambda}_{i}\right)_{0 \leq i \leq n} \in \mathbb{N} \times \prod_{i=0}^{n} \mathbb{N}^{N_{i}+1}\left|\lambda_{0} \leq D_{0}, \boldsymbol{\lambda}_{i}=\left(\lambda_{i, j}\right)_{0 \leq j \leq N_{i}},\right| \boldsymbol{\lambda}_{i} \mid=D_{i}\right\} .
$$

Soit $s \in E \otimes_{K} \mathbb{C}_{v_{0}}$. Il existe une unique famille $\left(p_{\boldsymbol{\lambda}}\right)_{\boldsymbol{\lambda} \in \Lambda} \in\left(\mathbb{C}_{v_{0}}\right)^{\text {card } \Lambda}$ telle que pour tout $z \in \mathbb{C}_{v_{0}} \times \mathscr{U}$, on ait

$$
F_{s}(z)=\sum_{\lambda \in \Lambda} p_{\boldsymbol{\lambda}} P_{\lambda_{0}}\left(z_{0}\right) \prod_{i=1}^{n} \prod_{j=0}^{N_{i}} \varphi_{i, j}^{\lambda_{i, j}}\left(z_{i}\right)
$$

et $\|s\|_{E, v_{0}}=\left|\left(p_{\boldsymbol{\lambda}}\right)_{\boldsymbol{\lambda}}\right|_{v_{0}}$. Pour tout $\boldsymbol{\lambda} \in \Lambda$, on note

$$
\Psi_{i}^{\boldsymbol{\lambda}_{i}}=\prod_{j=0}^{N_{i}} \varphi_{i, j}^{\lambda_{i, j}} \quad \text { et } \quad F_{s_{\boldsymbol{\lambda}}}=P_{\lambda_{0}} \prod_{i=1}^{n} \Psi_{i}^{\boldsymbol{\lambda}_{i}}
$$

Supposons que la place $v_{0}$ est archimédienne. Soit $\mathbf{e}^{\prime}$ une base orthonormée de $t_{G_{0} \times G}\left(\mathbb{C}_{v_{0}}\right)$ obtenue par concaténation de bases des espaces vectoriels $t_{G_{i}}\left(\mathbb{C}_{v_{0}}\right), 0 \leq i \leq n$. La base $\mathbf{e}^{\prime}$ permet d'identifier $t_{G_{0} \times G}\left(\mathbb{C}_{v_{0}}\right)$ à $\mathbb{C}_{v_{0}} \times \mathbb{C}_{v_{0}}^{g_{1}} \times \cdots \times \mathbb{C}_{v_{0}}^{g_{n}}$. D'après le lemme 8.8, on a

$$
\begin{aligned}
\left|\frac{1}{\boldsymbol{\tau} !} \mathcal{D}_{\mathbf{v}}^{\boldsymbol{\tau}} F_{s}(z)\right|_{v_{0}} & \leq\left(g^{2}-1\right)^{|\boldsymbol{\tau}|} \max _{\substack{|\boldsymbol{\sigma}|=|\boldsymbol{\tau}| \\
\sigma_{0}=\tau_{0}}}\left|\sum_{\boldsymbol{\lambda} \in \Lambda} p_{\boldsymbol{\lambda}} \frac{1}{\boldsymbol{\sigma} !} \mathcal{D}_{\mathbf{e}^{\prime}}^{\boldsymbol{\sigma}} F_{s_{\boldsymbol{\lambda}}}(z)\right|_{v_{0}} \\
& \leq\left(g^{2}-1\right)^{|\boldsymbol{\tau}|}\|s\|_{E, v_{0}} \operatorname{card} \Lambda \max _{\substack{|\boldsymbol{\sigma}|=|\boldsymbol{\tau}| \\
\sigma=1 \\
\boldsymbol{\lambda} \in \Lambda}}\left|\frac{1}{\boldsymbol{\sigma} !} \mathcal{D}_{\mathbf{e}^{\prime}}^{\boldsymbol{\sigma}} F_{s_{\boldsymbol{\lambda}}}(z)\right|_{v_{0}} .
\end{aligned}
$$

Le cardinal de $\Lambda$ est inférieur à $D_{0}^{\prime}\left(D_{1}^{\prime}\right)^{N_{1}} \cdots\left(D_{n}^{\prime}\right)^{N_{n}}$. Soit $\boldsymbol{\lambda} \in \Lambda$ et $\boldsymbol{\sigma} \in \mathbb{N}^{g+1-t}$. On a

$$
\frac{1}{\boldsymbol{\sigma} !} \mathcal{D}_{\mathbf{e}^{\prime}}^{\sigma^{\prime}} F_{s_{\boldsymbol{\lambda}}}(z)=\frac{P_{\lambda_{0}}^{\left(\sigma_{0}\right)}}{\sigma_{0} !}\left(z_{0}\right) \prod_{i=1}^{n}\left(\left(\prod_{j=1}^{g_{i}} \frac{1}{\sigma_{i, j} !}\left(\frac{\partial}{\partial z_{i, j}}\right)^{\sigma_{i, j}}\right) \prod_{k=0}^{N_{i}} \varphi_{i, k}^{\lambda_{i, k}}\left(z_{i}\right)\right) .
$$

Soit $\ell \in\{1, \ldots, n\}$. Pour $\boldsymbol{\theta}=\left(\theta_{1}, \ldots, \theta_{g_{\ell}}\right) \in[0,2 \pi]^{g_{\ell}}$, on note $z_{\boldsymbol{\theta}}=\left(e^{i \theta_{1}}, \ldots, e^{i \theta_{g_{\ell}}}\right) \in \mathbb{C}_{v_{0}}^{g_{\ell}}$. D'après l'inégalité de Cauchy, on a

$$
\begin{aligned}
\left|\prod_{j=1}^{g_{\ell}} \frac{1}{\sigma_{\ell, j} !}\left(\frac{\partial}{\partial z_{\ell, j}}\right)^{\sigma_{\ell, j}} \prod_{k=0}^{N_{\ell}} \varphi_{\ell, k}^{\lambda_{\ell, k}}\left(z_{\ell}\right)\right|_{v_{0}} & \leq \sup _{\boldsymbol{\theta} \in[0,2 \pi]^{g_{\ell}}}\left|\prod_{k=0}^{N_{\ell}} \varphi_{\ell, k}^{\lambda_{\ell, k}}\left(z_{\ell}+z_{\boldsymbol{\theta}}\right)\right|_{v_{0}} \\
& \leq \sup _{\boldsymbol{\theta}, k}\left|\varphi_{\ell, k}\left(z_{\ell}+z_{\boldsymbol{\theta}}\right)\right|_{v_{0}}^{D_{\ell}} \\
& \leq \exp \left(q_{20} D_{\ell}\left(1+\left\|z_{\ell}\right\|_{v_{0}}+\left\|z_{\boldsymbol{\theta}}\right\|_{v_{0}}\right)^{\rho_{\ell}}\right) \\
& \leq \exp \left(c D_{\ell}\left(1+\left\|z_{\ell}\right\|_{v_{0}}\right)^{\rho_{\ell}}\right)
\end{aligned}
$$

où $c \geq 1$ est une constante. On en déduit la majoration voulue si $v_{0}$ est archimédienne en posant $q_{27}=c+N_{i}$. Supposons maintenant que la place $v_{0}$ est finie et soit $z \in$ $\mathbb{C}_{v_{0}} \times D\left(0, r_{p_{0}}\right)$. On a dans ce cas 


$$
\left|\frac{1}{\boldsymbol{\tau} !} \mathcal{D}_{\mathbf{v}}^{\boldsymbol{\tau}} F_{s}(z)\right|_{v_{0}} \leq\|s\|_{E, v_{0}} \max _{\substack{\boldsymbol{\sigma}|=| \boldsymbol{\tau} \mid \\ \sigma=1 \\ \boldsymbol{\lambda} \in \Lambda}}\left|\frac{1}{\boldsymbol{\sigma} !} \mathcal{D}_{\left(e_{0}, \mathbf{e}\right)}^{\boldsymbol{\sigma}} F_{s_{\boldsymbol{\lambda}}}(z)\right|_{v_{0}}
$$

où la base e a été définie à la page 17. Par choix de la base e, on connaît un développement en série entière à coefficients dans $\mathscr{O}_{K_{v_{0}}}$ de $\prod_{i=1}^{n} \Psi^{\boldsymbol{\lambda}_{i}}$ pour tout $\boldsymbol{\lambda} \in \Lambda$, convergeant sur $D\left(0, r_{p_{0}}\right)$ : pour tout $\boldsymbol{\lambda} \in \Lambda$, il existe $\left(a_{\mathbf{n}, \boldsymbol{\lambda}}\right)_{\mathbf{n} \in \mathbb{N}^{g}} \in \mathscr{O}_{K_{v_{0}}}^{\mathbb{N}^{g}}$ tel que

$$
F_{s_{\boldsymbol{\lambda}}}(\mathbf{z})=P_{\lambda_{0}}\left(z_{0}\right) \sum_{\mathbf{n} \in \mathbb{N}^{g}} \frac{a_{\mathbf{n}, \boldsymbol{\lambda}}}{\mathbf{n} !} \mathbf{z}_{1}^{\mathbf{n}}, \quad \forall \mathbf{z}=\left(z_{0}, \mathbf{z}_{1}\right) \in \mathbb{C}_{v_{0}} \times D\left(0, r_{p_{0}}\right) .
$$

On en déduit que pour tout $\left(z_{0}, \mathbf{z}_{1}\right) \in \mathbb{C}_{v_{0}} \times D\left(0, r_{p_{0}}\right)$ et tout $\boldsymbol{\sigma}=\left(\sigma_{0}, \boldsymbol{\sigma}_{1}\right)=\left(\sigma_{0}, \sigma_{1}, \ldots, \sigma_{g}\right)$ $\in \mathbb{N}^{g+1}$, on a

$$
\frac{1}{\boldsymbol{\sigma} !} \mathcal{D}_{\left(e_{0}, \mathbf{e}\right)}^{\boldsymbol{\sigma}} F_{s_{\boldsymbol{\lambda}}}\left(z_{0}, \mathbf{z}_{1}\right)=\frac{P_{\lambda_{0}}^{\left(\sigma_{0}\right)}}{\sigma_{0} !}\left(z_{0}\right) \sum_{\mathbf{n}} \frac{a_{\mathbf{n}, \boldsymbol{\lambda}}}{\left(\mathbf{n}-\boldsymbol{\sigma}_{1}\right) !} \mathbf{z}_{1}^{\mathbf{n}-\boldsymbol{\sigma}_{1}}
$$

où $\mathbf{n}$ parcourt l'ensemble $\left\{\left(n_{1}, \ldots, n_{g}\right) \in \mathbb{N}^{g} \mid n_{j} \geq \sigma_{j} \forall 1 \leq j \leq g\right\}$. La majoration $r_{p_{0}}^{|\mathbf{n}|} \leq|\mathbf{n} !|_{v_{0}}$ pour tout $\mathbf{n} \in \mathbb{N}^{g}$ entraîne alors

$$
\left|\frac{1}{\boldsymbol{\sigma} !} \mathcal{D}_{\left(e_{0}, \mathbf{e}\right)}^{\boldsymbol{\sigma}} F_{s_{\boldsymbol{\lambda}}}(z)\right|_{v_{0}} \leq\left|\frac{P_{\lambda_{0}}^{\left(\sigma_{0}\right)}}{\sigma_{0} !}\left(z_{0}\right)\right|_{v_{0}},
$$

ce qui démontre le lemme.

Nous pouvons maintenant énoncer la majoration de $\left\|\mathcal{A}_{0}\right\|_{v_{0}}$ dont nous aurons besoin par la suite.

Proposition 8.10. Si $v_{0}$ est une place archimédienne, il existe une constante $q 28 \geq 1$ telle que

$$
\left\|\mathcal{A}_{0}\right\|_{v_{0}} \leq \exp \left(\frac{q 28}{C_{0}}\right) \max _{\substack{\lambda_{0} \leq D_{0} \\ h \in \mathbb{N}, m \leq S_{0} \\ h \leq 2(g+1) T}}\left|\frac{P_{\lambda_{0}}^{(h)}}{h !}(m)\right|_{v_{0}} .
$$

Si la place $v_{0}$ est ultramétrique, alors

$$
\left\|\mathcal{A}_{0}\right\|_{v_{0}} \leq \max _{\substack{\lambda_{0} \leq D_{0} \\ m \in \mathbb{N}, m \leq S_{0} \\ h \leq 2(g+1) T}}\left|\frac{P_{\lambda_{0}}^{(h)}}{h !}(m)\right|_{v_{0}}
$$

Démonstration. Supposons que la place $v_{0}$ est archimédienne. D'après la définition de $\left\|\mathcal{A}_{0}\right\|_{v_{0}}$ et le lemme 8.9 (et par une majoration grossière de card $\boldsymbol{\Upsilon}$ ), il existe une constante $c$ telle que

$$
\left\|\mathcal{A}_{0}\right\|_{v_{0}} \leq S_{0} e^{4\left(g^{2}-1\right) T} D_{0}^{\prime} \prod_{i=1}^{n} \exp \left(q_{27} D_{i}^{\prime}\left(1+S_{0}\left\|u_{i}\right\|_{v_{0}}\right)^{\rho_{i}}\right) \max _{\substack { \lambda_{0} \leq D_{0} \\
\begin{subarray}{c}{m \in \mathbb{N}, m \leq S_{0} \\
h \leq 2(g+1) T{ \lambda _ { 0 } \leq D _ { 0 } \\
\begin{subarray} { c } { m \in \mathbb { N } , m \leq S _ { 0 } \\
h \leq 2 ( g + 1 ) T } }\end{subarray}}\left|\frac{P_{\lambda_{0}}^{(h)}}{h !}(m)\right|_{v_{0}} .
$$

Par ailleurs, le lemme 8.5 entraîne les inégalités

$$
\left.S_{0} \leq e^{S_{0}} \leq e^{U / \widetilde{T}} \leq e^{U / C_{0}}, \quad D_{0}^{\prime} \prod_{i=1}^{n} \exp \left(q_{27} D_{i}^{\prime}\left(1+S_{0}\left\|u_{i}\right\|_{v_{0}}\right)^{\rho_{i}}\right) \leq e^{(1+n q 27]}\right) U / C_{0}
$$


et on a

$$
e^{4\left(g^{2}-1\right) T} \leq \exp \left(4\left(g^{2}-1\right) U /\left[C_{0} \mathfrak{a}\right]\right) \leq \exp \left(8\left(g^{2}-1\right) U / C_{0}\right) .
$$

On en déduit que

$$
\left\|\mathcal{A}_{0}\right\|_{v_{0}} \leq \exp \left(\frac{c U}{C_{0}}\right) \max _{\substack{\lambda_{0} \leq D_{0} \\ m \in \mathbb{N}, m \leq S_{0} \\ h \leq 2(g+1) T}}\left|\frac{P_{\lambda_{0}}^{(h)}}{h !}(m)\right|_{v_{0}},
$$

où $c=2+8\left(g^{2}-1\right)+n q 27$. Si la place $v_{0}$ est finie, le lemme est une conséquence immédiate de la définition de $\left\|\mathcal{A}_{0}\right\|_{v_{0}}$ et du lemme 8.9 .

8.6. Construction d'une section auxiliaire. Les majorations du paragraphe 8.5 vont nous permettre de construire un élément non nul de $E \otimes_{K} \overline{\mathbb{Q}}$ de «petite » hauteur en appliquant le lemme de Siegel approché du paragraphe 2 .

Proposition 8.11. Il existe une constante $q_{29}$ et une section $s \in E \otimes_{K} \overline{\mathbb{Q}} \backslash\{0\}$ telle que

$$
h_{\alpha}(s) \leq \frac{q 29}{D \sqrt{C_{0}}}\left(U+\max _{\substack{\lambda_{0} \leq D_{0} \\ m \in \mathbb{N}, m \leq S_{0} \\ h \leq 2(g+1) T}} \log \left|\frac{P_{\lambda_{0}}^{(h)}}{h !}(m)\right|_{v_{0}}\right) .
$$

Démonstration. D'après le lemme 2.14 (page 15), il existe une section non nulle $s \in$ $E \otimes_{K} \overline{\mathbb{Q}}$ telle que

$$
h_{\alpha}(s) \leq \frac{\left[K_{v_{0}}: \mathbb{Q}_{v_{0}}\right]}{[K: \mathbb{Q}]} \times \frac{\operatorname{rg} \mathcal{A}_{0}}{\operatorname{dim} E}\left(\log _{+}\left(2 \alpha\left\|\mathcal{A}_{0}\right\|_{v_{0}}\right)\right)+\frac{1}{2} \log (\operatorname{dim} E)-\widehat{\mu}_{\mathrm{n}}(E) .
$$

Avec notre choix de normes $\left(\|\cdot\|_{E, v}\right)_{v \in \Sigma_{K}}$, la pente $\widehat{\mu}_{\mathrm{n}}(E)$ est nulle. De plus, $\operatorname{dim} E \leq$ $D_{0}^{\prime}\left(D_{1}^{\prime}\right)^{g_{1}} \cdots\left(D_{n}^{\prime}\right)^{g_{n}}$. D'après les lemmes 8.5 et 8.6 on a aussi

$$
\frac{1}{2} \log (\operatorname{dim} E) \leq \frac{1}{2}\left(\log D_{0}^{\prime}+\sum_{i=1}^{n} g_{i} \log D_{i}^{\prime}\right) \leq \frac{g+1}{2} \log \frac{U}{D C_{0}} \leq \frac{g+1}{2} \times \frac{U}{D C_{0}} .
$$

Par ailleurs, on a $\log \alpha=\left(\sqrt{C_{0}}+g+1\right) U$. D'après les propositions 8.7 et 8.10 , l'inégalité 8.9 entraîne

$$
h_{\alpha}(s) \leq \frac{2 q 25}{D C_{0}}\left(\left(\sqrt{C_{0}}+2(g+1)\right) U+\frac{q 28}{C_{0}}+\max _{\substack{\lambda_{0} \leq D_{0} \\ m \in \mathbb{N}, m \leq S_{0} \\ h \leq 2(g+1) T}} \log \left|\frac{P_{\lambda_{0}}^{(h)}}{h !}(m)\right|_{v_{0}}\right),
$$

ce qui implique la conclusion de la proposition.

REMARQUe 8.12. Le choix de la structure de fibré adélique hermitien sur $E$ intervient dans l'inégalité 8.9 (où apparaît la pente $\widehat{\mu}_{\mathrm{n}}(E)$ ), mais aussi dans les paragraphes suivants, où nous devons estimer la hauteur d'un jet. Il s'avère que dans notre démonstration, un choix différent de structure de fibré adélique hermitien sur $E$ ne modifie pas la minoration finale obtenue, à constante près. Comme nous n'expliciterons pas les constantes intervenant dans les théorèmes du paragraphe 4 nous avons défini les normes de façon à simplifier les calculs ultérieurs au maximum. 
8.7. Construction d'un jet et premières estimations. Considérons un élément non nul $s \in E \otimes_{K} \overline{\mathbb{Q}}$ dont la hauteur $h_{\alpha}(s)$ vérifie la majoration de la proposition 8.11. Soit $(m, \ell) \in \mathbb{N} \times \mathbb{N}$ le couple minimal (pour l'ordre lexicographique) pour lequel il existe un élément $\boldsymbol{\tau}$ de $\mathbb{N}^{g+1-t}$ tel que $|\boldsymbol{\tau}|=\ell$ et $\mathcal{D}_{\mathbf{w}}^{\boldsymbol{\tau}} F_{s}(m, m u) \neq 0$. D'après le lemme 8.4. on a $m \leq(g+1) S$ et $\ell \leq(g+1) T$. Soit $K^{\prime}$ une extension finie de $K$ telle que $s \in E \otimes_{K} K^{\prime}$. Pour tout $i \in\{1, \ldots, n\}$, on choisit un entier $\varepsilon_{i} \in\left\{0, \ldots, N_{i}\right\}$ vérifiant

$$
\left|\varphi_{i, \varepsilon_{i}}\left(m u_{i}\right)\right|_{v_{0}}=\max _{0 \leq j \leq N_{i}}\left|\varphi_{i, j}\left(m u_{i}\right)\right|_{v_{0}} .
$$

En particulier, on a $\varphi_{i, \varepsilon_{i}}\left(m u_{i}\right) \neq 0$. Soit $\left(b_{1}, \ldots, b_{g-t}\right)$ une $K$-base de $V$; on considère la base $\mathbf{b}$ de $W$ donnée par $\mathbf{b}=\left(b_{0}=e_{0}, b_{1}, \ldots, b_{g-t}\right)$. Dans ces conditions, on dispose du résultat suivant :

Lemme 8.13 ([21, lemme 4.10]). Pour tout $\boldsymbol{\tau} \in \mathbb{N}^{g+1-t}$ tel que $|\boldsymbol{\tau}|=\ell$, le coefficient de Taylor «tordu»

$$
\prod_{i=1}^{n} \varphi_{i, \varepsilon_{i}}\left(m u_{i}\right)^{-D_{i}} \frac{1}{\boldsymbol{\tau} !} \mathcal{D}_{\mathbf{b}}^{\boldsymbol{\tau}} F_{s}(m, m u)
$$

appartient $\grave{a} K^{\prime}$.

Démonstration. Nous reprenons les arguments de 21 . Soit $\left(p_{\boldsymbol{\lambda}}\right)_{\boldsymbol{\lambda} \in \Lambda}$ une famille d'éléments de $K^{\prime}$ telle que

$$
F_{s}=\sum_{\lambda \in \Lambda} p_{\lambda} P_{\lambda_{0}} \prod_{i, j} \varphi_{i, j}^{\lambda_{i, j}}
$$

et notons $Q$ le polynôme défini par

$$
Q\left(\mathbf{X}_{1}, \ldots, \mathbf{X}_{n}\right)=\sum_{\boldsymbol{\lambda} \in \Lambda} p_{\boldsymbol{\lambda}} \frac{P_{\lambda_{0}}^{\left(\tau_{0}\right)}}{\tau_{0} !}(m) \prod_{i=1}^{n} A^{(i)}\left(\Theta_{i, \varepsilon_{i}}\left(m u_{i}\right), \mathbf{X}_{i}\right)^{\boldsymbol{\lambda}_{i}}
$$

Par la formule de Leibniz et la définition de $\ell$, on a

$$
\prod_{i=1}^{n} \varphi_{i, \varepsilon_{i}}\left(m u_{i}\right)^{-D_{i}} \frac{1}{\boldsymbol{\tau} !} \mathcal{D}_{\mathbf{b}}^{\boldsymbol{\tau}} F_{s}(m, m u)=\left.\frac{1}{\boldsymbol{\tau} !} \mathcal{D}_{\mathbf{b}}^{\boldsymbol{\tau}}\left(\frac{F_{s}\left(m+z_{0}, m u+z\right)}{\prod_{i=1}^{n} \varphi_{i, \varepsilon_{i}}\left(m u_{i}+z_{i}\right)^{D_{i}}}\right)\right|_{\left(z_{0}, z\right)=(0,0)}
$$

où $z_{i}$ désigne la projection de $z \in t_{G}\left(\mathbb{C}_{v_{0}}\right)$ sur $t_{G_{i}}\left(\mathbb{C}_{v_{0}}\right)$. Par définition et par homogénéité des polynômes $A_{j}^{(i)}$, on a

$$
\begin{aligned}
\frac{F_{s}\left(m+z_{0}, m u+z\right)}{\prod_{i=1}^{n} \varphi_{i, \varepsilon_{i}}\left(m u_{i}+z_{i}\right)^{D_{i}}} & =\sum_{\boldsymbol{\lambda} \in \Lambda} p_{\boldsymbol{\lambda}} P_{\lambda_{0}}\left(m+z_{0}\right) \prod_{i, j}\left(\frac{\varphi_{i, j}}{\varphi_{i, \varepsilon_{i}}}\left(m u_{i}+z_{i}\right)\right)^{\lambda_{i, j}} \\
& =\sum_{\boldsymbol{\lambda} \in \Lambda} p_{\boldsymbol{\lambda}} P_{\lambda_{0}}\left(m+z_{0}\right) \prod_{i, j}\left(\frac{A_{j}^{(i)}\left(\Psi_{i}\left(m u_{i}\right), \Psi_{i}\left(z_{i}\right)\right)}{A_{\varepsilon_{i}}^{(i)}\left(\Psi_{i}\left(m u_{i}\right), \Psi_{i}\left(z_{i}\right)\right)}\right)^{\lambda_{i, j}} \\
& =\sum_{\boldsymbol{\lambda} \in \Lambda} p_{\boldsymbol{\lambda}} P_{\lambda_{0}}\left(m+z_{0}\right) \prod_{i, j}\left(\frac{A_{j}^{(i)}\left(\Theta_{i, \varepsilon_{i}}\left(m u_{i}\right), \Psi_{i}\left(z_{i}\right)\right)}{A_{\varepsilon_{i}}^{(i)}\left(\Theta_{i, \varepsilon_{i}}\left(m u_{i}\right), \Psi_{i}\left(z_{i}\right)\right)}\right)^{\lambda_{i, j}}
\end{aligned}
$$




$$
\begin{aligned}
= & \sum_{\boldsymbol{\lambda} \in \Lambda} p_{\boldsymbol{\lambda}} P_{\lambda_{0}}\left(m+z_{0}\right) \prod_{i, j} A_{j}^{(i)}\left(\Theta_{i, \varepsilon_{i}}\left(m u_{i}\right), \Psi_{i}\left(z_{i}\right)\right)^{\lambda_{i, j}} \\
& \times \frac{1}{\prod_{i=1}^{n} A_{\varepsilon_{i}}^{(i)}\left(\Theta_{i, \varepsilon_{i}}\left(m u_{i}\right), \Psi_{i}\left(z_{i}\right)\right)^{D_{i}}} .
\end{aligned}
$$

En dérivant par rapport à $z_{0}$, on a

$$
\left.\frac{\mathcal{D}_{b_{0}}^{\tau_{0}}}{\tau_{0} !}\left(\frac{F_{s}\left(m+z_{0}, m u+z\right)}{\prod_{i=1}^{n} \varphi_{i, \varepsilon_{i}}\left(m u_{i}+z_{i}\right)^{D_{i}}}\right)\right|_{z_{0}=0}=\frac{F_{Q}(z)}{\prod_{i=1}^{n} A_{\varepsilon_{i}}^{(i)}\left(\Theta_{i, \varepsilon_{i}}\left(m u_{i}\right), \Psi_{i}\left(z_{i}\right)\right)^{D_{i}}},
$$

où $F_{Q}=Q \circ \Psi$. En particulier, la dérivée $D_{\mathbf{b}}^{\boldsymbol{\sigma}} F_{Q}(0)$ est nulle pour tout $|\boldsymbol{\sigma}|<\ell-\tau_{0}$. On déduit alors de la formule de Leibniz l'égalité

$$
\begin{aligned}
\prod_{i=1}^{n} \varphi_{i, \varepsilon_{i}}\left(m u_{i}\right)^{-D_{i}} \frac{1}{\tau !} \mathcal{D}_{\mathbf{b}}^{\tau} F_{s}(m, m u) & \frac{1}{\prod_{i=1}^{n} A_{\varepsilon_{i}}^{(i)}\left(\Theta_{i, \varepsilon_{i}}\left(m u_{i}\right),(1: 0: \cdots: 0)\right)^{D_{i}}} \frac{\mathcal{D}_{b_{1}}^{\tau_{1}} \cdots \mathcal{D}_{b_{g-t}}^{\tau_{g-t}}}{\tau_{1} ! \cdots \tau_{g-t} !} F_{Q}(0) .
\end{aligned}
$$

Rappelons que les polynômes $A^{(i)}$ sont à coefficients dans $K$ et que $\Theta_{i, \varepsilon_{i}}\left(m u_{i}\right) \in K$ pour tout $i \in\{1, \ldots, n\}$. Ainsi,

$$
F_{Q}=Q \circ \Psi=\sum_{\boldsymbol{\lambda} \in \Lambda} p_{\boldsymbol{\lambda}} \frac{\left.P_{\lambda_{0}}^{\left(\tau_{0}\right.}\right)}{\tau_{0} !}(m) \prod_{i=1}^{n} A^{(i)}\left(\Theta_{i, \varepsilon_{i}}\left(m u_{i}\right), \Psi_{i}\right)^{\boldsymbol{\lambda}_{i}}
$$

est un élément de l'anneau $K^{\prime}\left[\left(\varphi_{i, j}\right)_{1 \leq i \leq n, 0 \leq j \leq N_{i}}\right]$. Comme

$$
\Psi_{i}(0)=\left(\varphi_{i, 0}(0), \ldots, \varphi_{i, N_{i}}(0)\right)=(1,0, \ldots, 0)
$$

et que l'anneau $K\left[\left(\varphi_{i, j} / \varphi_{i, 0}\right)_{1 \leq i \leq n, 0 \leq j \leq N_{i}}\right]$ est stable par dérivation, le membre de droite de 8.10 est clairement un élément de $K^{\prime}$. Le lemme est donc démontré.

On note $\mathbf{b}^{\vee}$ la base duale de $\mathbf{b}$. On considère alors l'élément $\mathcal{J}$ du produit symétrique $S^{\ell}\left(W^{\vee}\right) \otimes_{K} K^{\prime}$ défini par

$$
\mathcal{J}=\sum_{\substack{\boldsymbol{\tau} \in \mathbb{N}^{g+1-t} \\|\boldsymbol{\tau}|=\ell}} \prod_{i=1}^{n} \varphi_{i, \varepsilon_{i}}\left(m u_{i}\right)^{-D_{i}} \frac{1}{\boldsymbol{\tau} !} \mathcal{D}_{\mathbf{b}}^{\boldsymbol{\tau}} F_{s}(m, m u)\left(\mathbf{b}^{\vee}\right)^{\boldsymbol{\tau}}
$$

Lemme 8.14. Le vecteur $\mathcal{J} \in S^{\ell}\left(W^{\vee}\right) \otimes_{K} K^{\prime}$ ne dépend pas du choix de la base $\mathbf{b}$.

Démonstration. Soient $\mathbf{b}_{1}$ et $\mathbf{b}_{2}$ deux bases de $W$ et soit $x \in W$. Pour tout $\lambda \in \mathbb{C}_{v_{0}}$ tel que le vecteur $(m, m u)+\lambda x$ de $t_{G_{0} \times G}\left(\mathbb{C}_{v_{0}}\right)$ appartienne à $\mathbb{C}_{v_{0}} \times \mathscr{U}$, on a

$$
\begin{aligned}
F_{s}((m, m u)+\lambda x) & =\sum_{k \in \mathbb{N}} \sum_{\substack{\boldsymbol{\tau} \in \mathbb{N}^{g+1-t} \\
|\boldsymbol{\tau}|=k}} \frac{1}{\boldsymbol{\tau} !} \mathcal{D}_{\mathbf{b}_{1}}^{\boldsymbol{\tau}} F_{s}(m, m u)\left(\mathbf{b}_{1}^{\vee}\right)^{\boldsymbol{\tau}}(\lambda x) \\
& =\sum_{k \in \mathbb{N}}\left(\sum_{\substack{\boldsymbol{\tau} \in \mathbb{N}^{g+1-t} \\
|\boldsymbol{\tau}|=k}} \frac{1}{\boldsymbol{\tau} !} \mathcal{D}_{\mathbf{b}_{1}}^{\boldsymbol{\tau}} F_{s}(m, m u)\left(\mathbf{b}_{1}^{\vee}\right)^{\boldsymbol{\tau}}(x)\right) \lambda^{k} .
\end{aligned}
$$


De même, on a

$$
F_{s}((m, m u)+\lambda x)=\sum_{k \in \mathbb{N}}\left(\sum_{\substack{\boldsymbol{\tau} \in \mathbb{N}^{g+1-t} \\|\boldsymbol{\tau}|=k}} \frac{1}{\boldsymbol{\tau} !} \mathcal{D}_{\mathbf{b}_{2}}^{\boldsymbol{\tau}} F_{s}(m, m u)\left(\mathbf{b}_{2}^{\vee}\right)^{\boldsymbol{\tau}}(x)\right) \lambda^{k} .
$$

On en déduit que pour tout $x \in W$ et tout $k \in \mathbb{N}$,

$$
\sum_{\substack{\boldsymbol{\tau} \in \mathbb{N}^{g+1-t} \\|\boldsymbol{\tau}|=k}} \frac{1}{\boldsymbol{\tau} !} \mathcal{D}_{\mathbf{b}_{1}}^{\boldsymbol{\tau}} F_{s}(m, m u)\left(\mathbf{b}_{1}^{\vee}\right)^{\boldsymbol{\tau}}(x)=\sum_{\substack{\boldsymbol{\tau} \in \mathbb{N}^{g+1-t} \\|\boldsymbol{\tau}|=k}} \frac{1}{\boldsymbol{\tau} !} \mathcal{D}_{\mathbf{b}_{2}}^{\boldsymbol{\tau}} F_{s}(m, m u)\left(\mathbf{b}_{2}^{\vee}\right)^{\boldsymbol{\tau}}(x),
$$

et donc

$$
\sum_{\substack{\boldsymbol{\tau} \in \mathbb{N}^{g+1-t} \\|\boldsymbol{\tau}|=k}} \frac{1}{\boldsymbol{\tau} !} \mathcal{D}_{\mathbf{b}_{1}}^{\boldsymbol{\tau}} F_{s}(m, m u)\left(\mathbf{b}_{1}^{\vee}\right)^{\boldsymbol{\tau}}=\sum_{\substack{\boldsymbol{\tau} \in \mathbb{N}^{g+1-t} \\|\boldsymbol{\tau}|=k}} \frac{1}{\boldsymbol{\tau} !} \mathcal{D}_{\mathbf{b}_{2}}^{\boldsymbol{\tau}} F_{s}(m, m u)\left(\mathbf{b}_{2}^{\vee}\right)^{\boldsymbol{\tau}},
$$

ce qui démontre le lemme.

On munit $S^{\ell}\left(W^{\vee}\right)$ de la structure de fibré adélique quotient de celle de $\left(W^{\vee}\right)^{\otimes \ell}$ induite par celle de $W$ (voir le paragraphe 2.3). Concrètement, si b est une base orthonormée de $W \otimes_{K} \mathbb{C}_{v}$ pour une place $v$ de $K^{\prime}$, alors

$$
\|\mathcal{J}\|_{v}^{2}=\sum_{|\boldsymbol{\tau}|=\ell} \frac{\boldsymbol{\tau} !}{\ell !}\left|\prod_{i=1}^{n} \varphi_{i, \varepsilon_{i}}\left(m u_{i}\right)^{-D_{i}} \frac{1}{\boldsymbol{\tau} !} \mathcal{D}_{\mathbf{b}}^{\boldsymbol{\tau}} F_{s}(m, m u)\right|_{v}^{2}
$$

si $v$ est archimédienne et

$$
\|\mathcal{J}\|_{v}=\max _{|\boldsymbol{\tau}|=\ell}\left|\prod_{i=1}^{n} \varphi_{i, \varepsilon_{i}}\left(m u_{i}\right)^{-D_{i}} \frac{1}{\boldsymbol{\tau} !} \mathcal{D}_{\mathbf{b}}^{\boldsymbol{\tau}} F_{s}(m, m u)\right|_{v}
$$

sinon. Remarquons $\tau ! / \ell ! \leq 1$ pour tout $\boldsymbol{\tau} \in \mathbb{N}^{g-t+1}$ tel que $|\boldsymbol{\tau}|=\ell(\operatorname{car} \ell ! / \tau ! \in \mathbb{N} \backslash\{0\}$ est un coefficient multinomial).

L'objectif de la suite du texte est d'obtenir un encadrement suffisamment fin de la hauteur de $\mathcal{J}$ afin d'aboutir à une contradiction. La négation de l'hypothèse 2 fournira alors une minoration de la distance $d(u, V)$ (sous l'hypothèse 1 ). Pour les places $v \in \Sigma_{K^{\prime}}$, $v \nmid v_{0}$, on a une majoration de $\|\mathcal{J}\|_{v}$ donnée par les résultats de [21, paragraphes 4.2 et 4.3], que nous allons rappeler maintenant. Nous obtiendrons une majoration de la quantité

$$
\sum_{\substack{v \in \Sigma_{K^{\prime}} \\ v \nmid v_{0}}} \frac{\left[K_{v}^{\prime}: \mathbb{Q}_{v}\right]}{\left[K^{\prime}: \mathbb{Q}\right]} \log \|\mathcal{J}\|_{v} .
$$

8.7.1. Estimations ultramétriques. Rappelons que nous avons a défini un modèle lisse $\mathfrak{G} \rightarrow \operatorname{Spec} \mathscr{O}_{K}[1 / \widetilde{m}]$ de $G$ au paragraphe 3 . Si $v$ est une place finie de $K^{\prime}$ ne divisant pas $\widetilde{m}$, on note $\widehat{\mathfrak{G}}_{v}$ (respectivement $\widehat{H}_{v}$ ) le complété formel à l'origine du schéma $\mathfrak{G} \times \operatorname{Spec} \mathscr{O}_{K_{v}^{\prime}}$ (respectivement du schéma $\left.H \times{ }_{K} \operatorname{Spec} K_{v}^{\prime}\right)$. De cette façon, $\widehat{H}_{v}$ est un sousschéma formel lisse du schéma formel $\widehat{G}_{K_{v}^{\prime}}:=\widehat{\mathfrak{G}}_{v} \widehat{\otimes} \operatorname{Spec} K_{v}^{\prime}$. On note alors $R_{\mathfrak{G}, v}\left(\widehat{H}_{v}\right)$ la taille de $\widehat{H}_{v}$ relativement au modèle $\widehat{\mathfrak{G}}_{v}$ de $\widehat{G}_{K_{v}^{\prime}}$, comme définie par Bost [8, paragraphe 3.1] (voir aussi [21, page 243]). On démontre alors la proposition suivante, qui est un cas particulier de la proposition 4.11 de [21]. 
Proposition 8.15. Soit $v \in \Sigma_{K^{\prime}}$ une place finie ne divisant pas $\widetilde{m}$. Alors

$$
\begin{aligned}
\|\mathcal{J}\|_{v} \leq & \|s\|_{E, v}\left(\prod_{i=1}^{n} \max _{0 \leq j \leq N_{i}}\left|\frac{\varphi_{i, j}}{\varphi_{i, \varepsilon_{i}}}\left(m u_{i}\right)\right|_{v}^{\llbracket 19 D^{D_{i}}}\right) \max _{\substack{\lambda_{0} \leq D_{0} \\
h \leq \ell}}\left|\frac{P_{\lambda_{0}}^{(h)}}{h !}(m)\right|_{v} \\
& \times R_{\mathfrak{G}, v}\left(\widehat{H}_{v}\right)^{-\ell} \prod_{i=1}^{n}\left|A_{\varepsilon_{i}}^{(i)}\left(\Theta_{i, \varepsilon_{i}}\left(m u_{i}\right),(1: 0: \cdots: 0)\right)\right|_{v}^{-D_{i}} .
\end{aligned}
$$

Si $v \in \Sigma_{K^{\prime}}$ divise $\widetilde{m}$, alors la même majoration reste vraie en remplaçant $R_{\mathfrak{G}, v}\left(\widehat{H}_{v}\right)^{-\ell}$ par $\frac{\ell+D_{1}+\cdots+D_{n}}{30}$, pour une certaine constante $\overline{130} \geq 1$.

Démonstration. Soit $\boldsymbol{\tau}=\left(\tau_{0}, \ldots, \tau_{g-t}\right) \in \mathbb{N}^{g-t}$ tel que $|\boldsymbol{\tau}|=\ell$. Considérons à nouveau le polynôme

$$
Q\left(\mathbf{X}_{1}, \ldots, \mathbf{X}_{n}\right)=\sum_{\boldsymbol{\lambda} \in \Lambda} p_{\boldsymbol{\lambda}} \frac{P_{\lambda_{0}}^{\left(\tau_{0}\right)}}{\tau_{0} !}(m) \prod_{i=1}^{n} A^{(i)}\left(\Theta_{i, \varepsilon_{i}}\left(m u_{i}\right), \mathbf{X}_{i}\right)^{\boldsymbol{\lambda}_{i}},
$$

où $\left(p_{\boldsymbol{\lambda}}\right)_{\boldsymbol{\lambda} \in \Lambda}$ est une famille d'éléments de $K^{\prime}$ telle que

$$
F_{s}=\sum_{\lambda \in \Lambda} p_{\lambda} P_{\lambda_{0}} \prod_{i, j} \varphi_{i, j}^{\lambda_{i, j}}
$$

$\mathrm{Au}$ cours de la démonstration du lemme 8.13 nous avons établi les égalités

$$
\left.\frac{\mathcal{D}_{b_{0}}^{\tau_{0}}}{\tau_{0} !}\left(\frac{F_{s}\left(m+z_{0}, m u+z\right)}{\prod_{i=1}^{n} \varphi_{i, \varepsilon_{i}}\left(m u_{i}+z_{i}\right)^{D_{i}}}\right)\right|_{z_{0}=0}=\frac{F_{Q}(z)}{\prod_{i=1}^{n} A_{\varepsilon_{i}}^{(i)}\left(\Theta_{i, \varepsilon_{i}}\left(m u_{i}\right), \Psi_{i}\left(z_{i}\right)\right)^{D_{i}}},
$$

où $F_{Q}=Q \circ \Psi_{v_{0}}$, et

$$
\begin{aligned}
\prod_{i=1}^{n} \varphi_{i, \varepsilon_{i}}\left(m u_{i}\right)^{-D_{i}} \frac{1}{\tau !} \mathcal{D}_{\mathbf{b}}^{\tau} F_{s}(m, m u) & \frac{1}{\prod_{i=1}^{n} A_{\varepsilon_{i}}^{(i)}\left(\Theta_{i, \varepsilon_{i}}\left(m u_{i}\right),(1: 0: \cdots: 0)\right)^{D_{i}}} \frac{\mathcal{D}_{b_{1}}^{\tau_{1}} \cdots \mathcal{D}_{b_{g-t}}^{\tau_{g-t}}}{\tau_{1} ! \cdots \tau_{g-t} !} F_{Q}(0) .
\end{aligned}
$$

Soit $v$ une place finie de $K^{\prime}$ et soit $i \in\{1, \ldots, n\}$. Les anneaux

$$
K\left[\left(\varphi_{v, i, j} / \varphi_{v, i, 0}\right)_{0 \leq j \leq N_{i}}\right], \quad K\left[\left(\varphi_{v_{0}, i, j} / \varphi_{v_{0}, i, 0}\right)_{0 \leq j \leq N_{i}}\right]
$$

sont stables par dérivation selon un vecteur de $t_{G_{i}}(K)$, et

$$
\left(\varphi_{v, i, 0}, \ldots, \varphi_{v, i, N_{i}}\right)(0)=\left(\varphi_{v_{0}, i, 0}, \ldots, \varphi_{v_{0}, i, N_{i}}\right)(0)=(1,0, \ldots, 0) .
$$

En appliquant les formules de dérivations de fonctions composées de plusieurs variables, on en déduit que

$$
\mathcal{D}_{b_{1}}^{\sigma_{1}} \cdots \mathcal{D}_{b_{g-t}}^{\sigma_{g-t}} F_{Q}(0)=\mathcal{D}_{b_{1}}^{\sigma_{1}} \cdots \mathcal{D}_{b_{g-t}}^{\sigma_{g-t}} F_{Q, v}(0)
$$

pour tout $\boldsymbol{\sigma} \in \mathbb{N}^{g-t}$, où $F_{Q, v}=Q \circ \Psi_{v}$ (rappelons que $F_{Q}=Q \circ \Psi_{v_{0}}$ ). En particulier les dérivées $\mathcal{D}_{b_{1}}^{\sigma_{1}} \cdots \mathcal{D}_{b_{g-t}}^{\sigma_{g-t}} F_{Q, v}(0)$ sont nulles pour tout $\boldsymbol{\sigma} \in \mathbb{N}^{g-t}$ tel que $|\boldsymbol{\sigma}|<\ell-\tau_{0}$. On en déduit que $F_{Q, v}$ satisfait les hypothèses du corollaire 4.5 de 21] (qui est une conséquence du lemme 3.3 de [8]) pour toute place finie $v$ ne divisant pas $\widetilde{m}$. D'après le lemme 8.14 on peut supposer sans perte de généralité que tous les vecteurs de la base $\mathbf{b}$ sont de 
norme $\|\cdot\|_{v}$ égale à 1 . Le corollaire 4.5 de 21 implique alors $\left({ }^{1}\right)$ que la valeur absolue $v$-adique de

$$
\frac{\mathcal{D}_{b_{1}}^{\tau_{1}} \cdots \mathcal{D}_{b_{g-t}}^{\tau_{g-t}}}{\tau_{1} ! \cdots \tau_{g-t} !} F_{Q}(0)=\frac{\mathcal{D}_{b_{1}}^{\tau_{1}} \cdots \mathcal{D}_{b_{g-t}}^{\tau_{g-t}}}{\tau_{1} ! \cdots \tau_{g-t} !} F_{Q, v}(0)
$$

est majorée par $R_{\mathfrak{G}, v}\left(\widehat{H}_{v}\right)^{-\ell} \max _{\boldsymbol{\lambda}}\left|q_{\boldsymbol{\lambda}}\right|_{v}$, où $\left(q_{\boldsymbol{\lambda}}\right)_{\boldsymbol{\lambda}}$ est la famille de coordonnées de $Q$ dans la base $\left(\mathbf{X}_{1}^{\boldsymbol{\lambda}_{1}} \cdots \mathbf{X}_{n}^{\boldsymbol{\lambda}_{n}}\right)_{\boldsymbol{\lambda}}\left(\boldsymbol{\lambda}\right.$ parcourt l'ensemble des $\left(\boldsymbol{\lambda}_{1}, \ldots, \boldsymbol{\lambda}_{n}\right) \in \mathbb{N}^{N_{1}} \times \cdots \times \mathbb{N}^{N_{n}}$ tels que $\left|\boldsymbol{\lambda}_{i}\right| \leq q_{19} D_{i}$ pour tout $i$ ). La première partie de la proposition découle alors d'une estimation immédiate des coefficients $\left(q_{\boldsymbol{\lambda}}\right)_{\boldsymbol{\lambda}}$ de $Q$, de l'égalité 8.12 et du fait que

$$
\|\mathcal{J}\|_{v} \leq \max _{|\boldsymbol{\tau}|=\ell}\left|\prod_{i=1}^{n} \varphi_{i, \varepsilon_{i}}\left(m u_{i}\right)^{-D_{i}} \frac{1}{\boldsymbol{\tau} !} \mathcal{D}_{\mathbf{b}}^{\boldsymbol{\tau}} F_{s}(m, m u)\right|_{v} .
$$

Le cas où $v \mid \widetilde{m}$ est une conséquence de l'égalité 8.12 et d'une estimation directe des

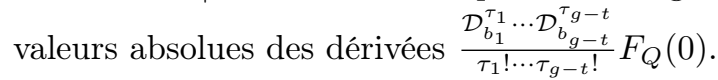

8.7.2. Estimations archimédiennes. Dans le cas archimédien, on a l'estimation suivante, qui correspond à la proposition 4.13 de [21].

Proposition 8.16. Il existe une constante q31 $\geq 1$ telle que pour toute place archimédienne $v \in \Sigma_{K^{\prime}}$, on ait

$$
\begin{aligned}
\|\mathcal{J}\|_{v} \leq & \frac{\ell+\log D_{0}+D_{1}+\cdots+D_{n}}{31}\|s\|_{E, v}\left(\prod_{i=1}^{n} \max _{0 \leq j \leq N_{i}}\left|\frac{\varphi_{i, j}}{\varphi_{i, \varepsilon_{i}}}\left(m u_{i}\right)\right|_{v}^{\mid 19 D^{D_{i}}}\right) \max _{\substack{\lambda_{0} \leq D_{0} \\
h \leq \ell}}\left|\frac{P_{\lambda_{0}}^{(h)}}{h !}(m)\right|_{v} \\
& \times \prod_{i=1}^{n}\left|A_{\varepsilon_{i}}^{(i)}\left(\Theta_{i, \varepsilon_{i}}\left(m u_{i}\right),(1: 0: \cdots: 0)\right)\right|_{v}^{-D_{i}} .
\end{aligned}
$$

Démonstration. La démonstration est analogue à celle de la seconde partie de la proposition 8.15, en remarquant que pour toute place archimédienne $v \in \Sigma_{K^{\prime}}$, on a

$$
\begin{aligned}
\left|\sum_{\boldsymbol{\lambda} \in \Lambda} p_{\boldsymbol{\lambda}} \frac{P_{\lambda_{0}}^{\left(\tau_{0}\right)}}{\tau_{0} !}(m)\right|_{v} & \leq\left.\max _{\substack{\lambda_{0} \leq D_{0} \\
h \leq \ell}}\left|\frac{P_{\lambda_{0}}^{(h)}(m)}{h !}\right|\left(p_{\boldsymbol{\lambda}}\right)_{\boldsymbol{\lambda}}\right|_{v} \sqrt{\operatorname{card} \Lambda} \\
& \leq \max _{\substack{\lambda_{0} \leq D_{0} \\
h \leq \ell}}\left|\frac{P_{\lambda_{0}}^{(h)}}{h !}(m)\right|_{v}\|s\|_{\alpha, v}\left(D_{0}^{\prime}\left(D_{1}^{\prime}\right)^{N_{1}} \cdots\left(D_{n}^{\prime}\right)^{N_{n}}\right)^{1 / 2},
\end{aligned}
$$

et

$$
\begin{aligned}
\|\mathcal{J}\|_{v} & \leq \operatorname{card}\left\{\boldsymbol{\tau} \in \mathbb{N}^{g+1-t}|| \boldsymbol{\tau} \mid=\ell\right\} \max _{|\boldsymbol{\tau}|=\ell}\left|\prod_{i=1}^{n} \varphi_{i, \varepsilon_{i}}\left(m u_{i}\right)^{-D_{i}} \frac{1}{\boldsymbol{\tau} !} \mathcal{D}_{\mathbf{b}}^{\boldsymbol{\tau}} F_{s}(m, m u)\right|_{v} \\
& \leq \ell^{g+1-t} \max _{|\boldsymbol{\tau}|=\ell}\left|\prod_{i=1}^{n} \varphi_{i, \varepsilon_{i}}\left(m u_{i}\right)^{-D_{i}} \frac{1}{\boldsymbol{\tau} !} \mathcal{D}_{\mathbf{b}}^{\boldsymbol{\tau}} F_{s}(m, m u)\right|_{v} .
\end{aligned}
$$

8.7.3. Estimation globale. Nous allons maintenant appliquer les propositions 8.15 et 8.16 pour obtenir une estimation portant sur toutes les places de $K^{\prime}$ ne divisant pas $v_{0}$.

$\left({ }^{1}\right)$ C'est ici qu'intervient l'hypothèse $t_{\mathfrak{G}} \otimes \mathscr{O}_{K_{v}}=\left\{z \in t_{G}\left(K_{v}\right) \mid\|z\|_{v} \leq 1\right\}$ faite sur les normes $\|\cdot\|_{v}$ pour $v \nmid \widetilde{m}$ (voir [21, page 243]). 
La démonstration nécessite une minoration des tailles de sous-schémas formels $R_{\mathfrak{G}, v}\left(\widehat{H}_{v}\right)$ due à Gaudron [21, qui est une conséquence d'un théorème de Raynaud [5, théorème 4, $\S 7.5]$.

Proposition 8.17. Il existe une constante $\overline{3_{22}} \geq 1$ telle que

$$
\begin{aligned}
& \sum_{\substack{v \in \Sigma_{K^{\prime}} \\
v \nmid v_{0}}} \frac{\left[K_{v}^{\prime}: \mathbb{Q}_{v}\right]}{\left[K^{\prime}: \mathbb{Q}\right]} \log \|\mathcal{J}\|_{v} \\
& \quad \leq \sum_{\substack{v \in \Sigma_{K^{\prime}} \\
v \nmid v_{0}}} \frac{\left[K_{v}^{\prime}: \mathbb{Q}_{v}\right]}{\left[K^{\prime}: \mathbb{Q}\right]}\left(\log \|s\|_{\alpha, v}+\max _{\substack{\lambda_{0} \leq D_{0} \\
h \leq(g+1) T}} \log \left|\frac{P_{\lambda_{0}}^{(h)}}{h !}(m)\right|_{v}\right)+q \frac{U}{D C_{0}} .
\end{aligned}
$$

Démonstration. D'après les propositions 8.15 et 8.16 , il existe une constante $c \geq 1$ telle que la quantité $\sum_{v \nmid v_{0}, v \in \Sigma_{K^{\prime}}} \frac{\left[K_{v}^{\prime}: \mathbb{Q}_{v}\right]}{\left[K^{\prime}: \mathbb{Q}\right]} \log \|\mathcal{J}\|_{v}$ soit majorée par

$$
\begin{aligned}
& \sum_{\substack{v \in \Sigma_{K^{\prime}} \\
v \nmid v_{0}}} \frac{\left[K_{v}^{\prime}: \mathbb{Q} v\right]}{\left[K^{\prime}: \mathbb{Q}\right]}\left\{\log \|s\|_{\alpha, v}+\max _{\substack{\lambda_{0} \leq D_{0} \\
h \leq(g+1) T}} \log \left|\frac{P_{\lambda_{0}}^{(h)}}{h !}(m)\right|_{v}\right. \\
& \left.+\sum_{i=1}^{n} D_{i}\left(c \log \max _{0 \leq j \leq N_{i}}\left|\frac{\varphi_{i, j}}{\varphi_{i, \varepsilon_{i}}}\left(m u_{i}\right)\right|_{v}-\log \left|A_{\varepsilon_{i}}^{(i)}\left(\Theta_{i, \varepsilon_{i}}\left(m u_{i}\right),(1: 0: \cdots: 0)\right)\right|_{v}\right)\right\} \\
& +c\left(T+\log D_{0}^{\prime}+\sum_{i=1}^{n} D_{i}\right)-\ell \sum_{v \in \Sigma^{\prime}} \frac{\left[K_{v}^{\prime}: \mathbb{Q}_{v}\right]}{\left[K^{\prime}: \mathbb{Q}\right]} \log R_{\mathfrak{G}, v}\left(\widehat{H}_{v}\right),
\end{aligned}
$$

où la dernière somme porte sur l'ensemble $\Sigma^{\prime} \subset \Sigma_{K^{\prime}}$ des places ultramétriques $v$ de $K^{\prime}$ ne divisant ni $v_{0}$ ni $\widetilde{m}$. D'après [21, page 245], quitte à agrandir l'extension $K^{\prime}$, il existe un sous-ensemble fini $F_{G} \subset \Sigma_{K^{\prime}}$, indépendant de $H$, tel que pour toute place $v \nmid \widetilde{m}$ de $K^{\prime}$, on ait :

- $R_{\mathfrak{G}, v}\left(\widehat{H}_{v}\right) \geq p^{-1 /(p-1)}$ si $v \mid p$ est dans $F_{G}$,

- $R_{\mathfrak{G}, v}\left(\widehat{H}_{v}\right)=1$ si $v \in \Sigma^{\prime} \backslash F_{G}$.

Notons $F_{G}^{\prime}$ l'ensemble des nombres premiers $p$ tels qu'il existe une place $v \in F_{G}$ divisant $p$. Alors $F_{G}^{\prime}$ ne dépend ni de $H$ ni de $K^{\prime}$ et on a

$$
\begin{aligned}
-\ell \sum_{v \in \Sigma^{\prime}} \frac{\left[K_{v}^{\prime}: \mathbb{Q}_{v}\right]}{\left[K^{\prime}: \mathbb{Q}\right]} \log R_{\mathfrak{G}, v}\left(\widehat{H}_{v}\right) & \leq \ell \sum_{v \in F_{G} \cap \Sigma^{\prime}, v \mid p} \frac{\left[K_{v}^{\prime}: \mathbb{Q}_{v}\right]}{\left[K^{\prime}: \mathbb{Q}\right]} \frac{1}{p-1} \log p \\
& =\ell \sum_{p \nmid \widetilde{m}, p \in F_{G}^{\prime}} \frac{1}{p-1} \log p \leq \ell \sum_{p \in F_{G}^{\prime}} \frac{1}{p-1} \log p .
\end{aligned}
$$

On en déduit que

$$
-\ell \sum_{v \in \Sigma^{\prime}} \frac{\left[K_{v}^{\prime}: \mathbb{Q}_{v}\right]}{\left[K^{\prime}: \mathbb{Q}\right]} \log R_{\mathfrak{G}, v}\left(\widehat{H}_{v}\right) \leq c^{\prime} T,
$$

où la constante $c^{\prime}:=(g+1)$ card $F_{G}^{\prime}$ est indépendante de $H$ et du corps $K^{\prime}$. Par ailleurs, pour tout $i \in\{1, \ldots, n\}, A_{\varepsilon_{i}}^{(i)}\left(\Theta_{i, \varepsilon_{i}}\left(m u_{i}\right),(1: 0: \cdots: 0)\right)$ appartient à $K$. La formule du produit et les propriétés de $A_{\varepsilon_{i}}^{(i)}$ rappelées au paragraphe 3 entraînent 


$$
\begin{aligned}
-\sum_{\substack{v \in \Sigma_{K^{\prime}} \\
v \nmid v_{0}}} \frac{\left[K_{v}^{\prime}: \mathbb{Q} v\right]}{\left[K^{\prime}: \mathbb{Q}\right]} \log \left|A_{\varepsilon_{i}}^{(i)}\left(\Theta_{i, \varepsilon_{i}}\left(m u_{i}\right),(1: 0: \cdots: 0)\right)\right|_{v} \\
=\sum_{\substack{v \in \Sigma_{K^{\prime}} \\
v \mid v_{0}}} \frac{\left[K_{v}^{\prime}: \mathbb{Q} v\right]}{\left[K^{\prime}: \mathbb{Q}\right]} \log \left|A_{\varepsilon_{i}}^{(i)}\left(\Theta_{i, \varepsilon_{i}}\left(m u_{i}\right),(1: 0: \cdots: 0)\right)\right|_{v} \\
=\frac{\left[K_{v_{0}}: \mathbb{Q}_{v_{0}}\right]}{[K: \mathbb{Q}]} \log \left|A_{\varepsilon_{i}}^{(i)}\left(\Theta_{i, \varepsilon_{i}}\left(m u_{i}\right),(1: 0: \cdots: 0)\right)\right|_{v_{0}} \\
\leq \frac{\left[K_{v_{0}}: \mathbb{Q} v_{0}\right]}{[K: \mathbb{Q}]} \log \left(e^{[19} \max _{j}\left|\frac{\varphi_{i, j}}{\varphi_{i, \varepsilon_{i}}}\left(m u_{i}\right)\right|_{v_{0}}^{[19}\right) \\
\leq \frac{\left[K_{v_{0}}: \mathbb{Q} v_{0}\right]}{[K: \mathbb{Q}]} \text { q19] } \quad\left(\operatorname{par} \operatorname{choix} \operatorname{de} \varepsilon_{i}\right) .
\end{aligned}
$$

De même, la formule du produit et le choix de $\varepsilon_{i}$ permettent d'écrire

$$
\begin{aligned}
\sum_{\substack{v \in \Sigma_{K^{\prime}} \\
v \nmid v_{0}}} \frac{\left[K_{v}^{\prime}: \mathbb{Q}_{v}\right]}{\left[K^{\prime}: \mathbb{Q}\right]} \log \max _{0 \leq j \leq N_{i}}\left|\frac{\varphi_{i, j}}{\varphi_{i, \varepsilon_{i}}}\left(m u_{i}\right)\right|_{v} & =\sum_{v \in \Sigma_{K^{\prime}}} \frac{\left[K_{v}^{\prime}: \mathbb{Q}_{v}\right]}{\left[K^{\prime}: \mathbb{Q}\right]} \log \max _{0 \leq j \leq N_{i}}\left|\varphi_{i, j}\left(m u_{i}\right)\right|_{v} \\
& =h\left(m \mathbf{p}_{i}\right) .
\end{aligned}
$$

Finalement, on obtient l'inégalité

$$
\begin{aligned}
\sum_{\substack{v \in \Sigma_{K^{\prime}} \\
v \nmid v_{0}}} \frac{\left[K_{v}^{\prime}: \mathbb{Q}_{v}\right]}{\left[K^{\prime}: \mathbb{Q}\right]} \log \|\mathcal{J}\|_{v} \leq & \sum_{\substack{v \in \Sigma_{K^{\prime}} \\
v \nmid v_{0}}} \frac{\left[K_{v}^{\prime}: \mathbb{Q}_{v}\right]}{\left[K^{\prime}: \mathbb{Q}\right]}\left(\log \|s\|_{\alpha, v}+\max _{\substack{\lambda_{0} \leq D_{0} \\
h \leq(g+1) T}} \log \left|\frac{P_{\lambda_{0}}^{(h)}}{h !}(m)\right|_{v}\right) \\
& +q \underline{32}\left(T+\log D_{0}^{\prime}+\sum_{i=1}^{n} D_{i} \max _{0 \leq k \leq(g+1) S}\left\{h\left(k \mathbf{p}_{i}\right)\right\}\right),
\end{aligned}
$$

et on en déduit la majoration voulue en appliquant les lemmes 8.5 et 8.6 selon que la place $v_{0}$ est archimédienne ou non.

Afin de contrôler la hauteur de $\mathcal{J}$, nous allons maintenant majorer $\|\mathcal{J}\|_{v}$ pour toute place $v$ de $K^{\prime}$ au-dessus de $v_{0}$. Afin d'aboutir à une contradiction, cette étape nécessite une étude minutieuse et fait l'objet du paragraphe suivant.

8.8. Extrapolation. Considérons une place quelconque de $K^{\prime}$ au-dessus de $v_{0}$, place que l'on note encore $v_{0}$. Nous cherchons ici à majorer la quantité

$$
\left|\prod_{i=1}^{n} \varphi_{i, \varepsilon_{i}}\left(m u_{i}\right)^{-D_{i}} \frac{1}{\boldsymbol{\tau} !} \mathcal{D}_{\mathbf{w}}^{\boldsymbol{\tau}} F_{s}(m, m u)\right|_{v_{0}}
$$

pour tout $\boldsymbol{\tau} \in \mathbb{N}^{g+1-t}$ tel que $|\boldsymbol{\tau}|=\ell$. Remarquons que si $(m, \boldsymbol{\tau})$ appartient à $\boldsymbol{\Upsilon}$, alors par définition de la norme $\|\cdot\|_{\alpha, v_{0}}$, on a

$$
\begin{aligned}
\left|\prod_{i=1}^{n} \varphi_{i, \varepsilon_{i}}\left(m u_{i}\right)^{-D_{i}} \frac{1}{\tau !} \mathcal{D}_{\mathbf{w}}^{\boldsymbol{\tau}} F_{s}(m, m u)\right|_{v_{0}} & \leq\|s\|_{\alpha, v_{0}} \prod_{i=1}^{n}\left|\varphi_{i, \varepsilon_{i}}\left(m u_{i}\right)\right|_{v_{0}}^{-D_{i}} \\
& \leq\|s\|_{\alpha, v_{0}} \prod_{i=1}^{n} \exp \left(q_{20} D_{i}\left(1+m\left\|u_{i}\right\|_{v_{0}}\right)^{\rho_{i}}\right) .
\end{aligned}
$$


Nous allons démontrer une majoration similaire dans le cas général. La méthode employée est classique en théorie des formes linéaires de logarithmes (et remonte à Baker); elle consiste en une extrapolation sur les points. Nous distinguerons les cas où la place $v_{0}$ est ultramétrique ou archimédienne, et nous utiliserons un lemme d'interpolation adapté à chacune de ces situations. Soit $\widetilde{u} \in V \otimes_{K} \mathbb{C}_{v_{0}}$ un vecteur tel que $d(u, V)=\|u-\widetilde{u}\|_{v_{0}}$.

8.8.1. Cas archimédien. Supposons que la place $v_{0}$ est archimédienne. Sans perte de généralité, nous supposons également que la base $\mathbf{w}$ de $W \otimes_{K} \mathbb{C}_{v_{0}}$ définie à la page 38 est orthonormée pour $\|\cdot\|_{v_{0}}$. Nous aurons besoin du lemme de comparaison suivant.

LEMME 8.18. Il existe une constante $\overline{33} \geq 1$ telle que pour tout entier naturel $k \leq(g+1) S$ et pour tout $\boldsymbol{\tau} \in \mathbb{N}^{g+1-t}$ tel que $|\boldsymbol{\tau}| \leq 2(g+1) T$, on ait

$$
\left|\frac{1}{\tau !} \mathcal{D}_{\mathbf{w}}^{\boldsymbol{\tau}} F_{s}(k, k u)-\frac{1}{\tau !} \mathcal{D}_{\mathbf{w}}^{\boldsymbol{\tau}} F_{s}(k, k \widetilde{u})\right|_{v_{0}} \leq \exp \left(\frac{q \sqrt{33} U}{C_{0}}\right) d(u, V)\|s\|_{\alpha, v_{0}} \max _{\substack{\lambda_{0} \leq D_{0} \\ h \leq \ell+1}}\left|\frac{P_{\lambda_{0}}^{(h)}}{h !}(k)\right|_{v_{0}} .
$$

Démonstration. Considérons l'application

$$
f:[0,1] \rightarrow \mathbb{C}_{v_{0}}, \quad \nu \mapsto \frac{1}{\boldsymbol{\tau} !} \mathcal{D}_{\mathbf{w}}^{\tau} F_{s}(k, k u+\nu k(\widetilde{u}-u)) .
$$

D'après l'inégalité des accroissements finis, on a $|f(1)-f(0)|_{v_{0}} \leq \sup _{\nu \in[0,1]}\left|f^{\prime}(\nu)\right|_{v_{0}}$. Soit $\mathbf{e}^{\prime}=\left(e_{0}, e_{1}^{\prime}, \ldots, e_{g}^{\prime}\right)$ une base orthonormée de $t_{G_{0} \times G}\left(\mathbb{C}_{v_{0}}\right)$. En posant

$$
u=\sum_{j=1}^{g} x_{j} e_{j}^{\prime} \quad \text { et } \quad \tilde{u}=\sum_{j=1}^{g} \widetilde{x}_{j} e_{j}^{\prime}
$$

on a

$$
f^{\prime}(\nu)=\sum_{j=1}^{g} k\left(\widetilde{x}_{j}-x_{j}\right) \frac{1}{\tau !} \frac{\partial}{\partial z_{j}} \mathcal{D}_{\mathbf{w}}^{\boldsymbol{\tau}} F_{s}(k, k u+\nu k(\widetilde{u}-u)) .
$$

En appliquant l'inégalité de Cauchy-Schwarz, on obtient

$$
\left|f^{\prime}(\nu)\right|_{v_{0}} \leq \sqrt{g} k\|\widetilde{u}-u\|_{v_{0}} \max _{1 \leq j \leq g}\left|\frac{1}{\tau !} \frac{\partial}{\partial z_{j}} \mathcal{D}_{\mathbf{w}}^{\boldsymbol{\tau}} F_{s}(k, k u+\nu k(\widetilde{u}-u))\right|_{v_{0}} .
$$

En reprenant les arguments des lemmes 8.8 et 8.9 , on en déduit qu'il existe une constante $c$ telle que

$$
\left|f^{\prime}(\nu)\right|_{v_{0}} \leq c^{T} k d(u, V) \max _{|\boldsymbol{\sigma}|=\ell+1}\left|\frac{1}{\boldsymbol{\sigma} !} \mathcal{D}_{\mathbf{e}^{\prime}}^{\boldsymbol{\sigma}} F_{s}(k, k u+\nu k(\widetilde{u}-u))\right|_{v_{0}},
$$

puis que la valeur absolue $\left|f^{\prime}(\nu)\right|_{v_{0}}$ est majorée par

$$
c^{T} k d(u, V)\|s\|_{\alpha, v_{0}} D_{0}^{\prime} \prod_{i=1}^{n} \exp \left(c D_{i}\left(1+k\left\|u_{i}+\nu\left(\widetilde{u}_{i}-u_{i}\right)\right\|_{v_{0}}\right)^{\rho_{i}}\right) \max _{\substack{\lambda_{0} \leq D_{0} \\ h \leq \ell+1}}\left|\frac{P_{\lambda_{0}}^{(h)}}{h !}(k)\right|_{v_{0}} .
$$

En remarquant que $\nu\left\|u_{i}-\widetilde{u}_{i}\right\|_{v_{0}} \leq d(u, V) \leq 1 / k$, on en déduit qu'il existe une constante $c^{\prime}$ telle que

$$
\left|f^{\prime}(\nu)\right|_{v_{0}} \leq c^{T} k d(u, V)\|s\|_{\alpha, v_{0}} D_{0}^{\prime} \prod_{i=1}^{n} \exp \left(c^{\prime} D_{i}\left(1+k\left\|u_{i}\right\|_{v_{0}}\right)^{\rho_{i}}\right) \max _{\substack{\lambda_{0} \leq D_{0} \\ h \leq \ell+1}}\left|\frac{P_{\lambda_{0}}^{(h)}}{h !}(k)\right|_{v_{0}} .
$$

On en déduit le résultat voulu en appliquant le lemme 8.5. 
Proposition 8.19. Il existe une constante $q_{34} \geq 1$ telle que si $C_{0} \geq q, 6$, alors pour tout $\boldsymbol{\tau} \in \mathbb{N}^{g+1-t}$ tel que $|\boldsymbol{\tau}|=\ell$, on a

$$
\begin{aligned}
& \left|\prod_{i=1}^{n} \varphi_{i, \varepsilon_{i}}\left(m u_{i}\right)^{-D_{i}} \frac{1}{\tau !} \mathcal{D}_{\mathbf{w}}^{\boldsymbol{\tau}} F_{s}(m, m u)\right|_{v_{0}} \\
& \quad \leq e^{-U}\|s\|_{\alpha, v_{0}} \max \left\{\left|\frac{P_{\lambda_{0}}^{(h)}}{h !}(z)\right|_{v_{0}}\left|h \leq 2(g+1) T, \lambda_{0} \leq D_{0},\right| z \mid \leq 2(g+1) S \mathfrak{e}\right\} .
\end{aligned}
$$

Démonstration. Considérons la fonction entière définie par $f(z)=\frac{1}{\boldsymbol{\tau} !} \mathcal{D}_{\mathbf{w}}^{\boldsymbol{\tau}} F_{s}(z, z \widetilde{u})$. En posant $S_{1}=S_{0}, T_{1}=(g+1) T, r=(g+1) S, R=2 r \mathfrak{e}$ et en appliquant le lemme d'interpolation archimédien 6.1 à $f$, on a

$$
|f(m)|_{v_{0}} \leq 2|f|_{R}\left(\frac{1}{\mathfrak{e}}\right)^{(g+1) T S_{0}}+5\left(\frac{9(g+1) S}{S_{0}}\right)^{(g+1) T S_{0}} \max _{\substack{t \in \mathbb{N}, t<S_{1} \\ h \leq T_{1}}}\left|\frac{f^{(h)}}{h !}(t)\right|_{v_{0}},
$$

où $|f|_{R}:=\sup \left\{|f(z)|_{v_{0}}|| z \mid \leq R\right\}$. Notons $\widetilde{\mathbf{x}}=\left(1, \widetilde{x}_{1}, \ldots, \widetilde{x}_{g-t}\right)$ les coordonnées de $(1, \widetilde{u})$ dans la base $\mathbf{w}$ :

$$
(1, \widetilde{u})=e_{0}+\sum_{j=1}^{n} \widetilde{x}_{j} w_{j}
$$

Pour tout entier $h \geq 0$ et tout $z \in t_{G_{0} \times G}\left(\mathbb{C}_{v_{0}}\right)$, on a

$$
\frac{1}{h !} f^{(h)}(z)=\sum_{\substack{|\mathbf{j}|=h \\
\mathbf{j} \in \mathbb{N}^{g+1-t}}}\left(\begin{array}{c}
\boldsymbol{\tau}+\mathbf{j} \\
\mathbf{j}
\end{array}\right) \widetilde{\mathbf{x}}^{\mathbf{j}} \frac{1}{(\boldsymbol{\tau}+\mathbf{j}) !} \mathcal{D}_{\mathbf{w}}^{\boldsymbol{\tau}+\mathbf{j}} F_{s}(z, z \widetilde{u})
$$

où $\widetilde{\mathbf{x}}^{\mathbf{j}}=\widetilde{x}_{1}^{j_{1}} \cdots \widetilde{x}_{g-t}^{j_{g-t}}$. Comme $d(u, V)=\|u-\widetilde{u}\|_{v_{0}} \leq 1$ et que la base $\mathbf{w}$ est orthonormée pour la place $v_{0}$, on en déduit qu'il existe une constante $c$ telle que pour tout entier $0 \leq h \leq(g+1) T$, on ait

$$
\left|\sum_{\substack{|\mathbf{j}|=h \\
\mathbf{j} \in \mathbb{N}^{g+1-t}}}\left(\begin{array}{c}
\boldsymbol{\tau}+\mathbf{j} \\
\mathbf{j}
\end{array}\right) \widetilde{\mathbf{x}}^{\mathbf{j}}\right| \leq \max \left\{1,\|\widetilde{u}\|_{v_{0}}\right\}^{h} h^{g+1-t} \max _{|\mathbf{j}|=h}\left|\left(\begin{array}{c}
\boldsymbol{\tau}+\mathbf{j} \\
\mathbf{j}
\end{array}\right)\right| \leq c^{T}\left(1+\|u\|_{v_{0}}\right)^{(g+1) T} .
$$

On en déduit qu'il existe une constante $c^{\prime}$ telle que pour tout entier $0 \leq h \leq(g+1) T$ et tout $z \in \mathbb{C}_{v_{0}}$, on ait

$$
\begin{aligned}
\left|\frac{f^{(h)}}{h !}(z)\right|_{v_{0}} & \leq c^{T}\left(1+\|u\|_{v_{0}}\right)^{(g+1) T} \max _{|\mathbf{j}|=h}\left|\frac{1}{(\boldsymbol{\tau}+\mathbf{j}) !} \mathcal{D}_{\mathbf{w}}^{\boldsymbol{\tau}+\mathbf{j}} F_{s}(z, z \widetilde{u})\right|_{v_{0}} \\
& \leq \exp \left(\frac{c^{\prime} U}{C_{0}}\right) \max _{|\mathbf{j}|=h}\left|\frac{1}{(\boldsymbol{\tau}+\mathbf{j}) !} \mathcal{D}_{\mathbf{w}}^{\boldsymbol{\tau}+\mathbf{j}} F_{s}(z, z \widetilde{u})\right|_{v_{0}} .
\end{aligned}
$$

Soient $h$ et $k$ des entiers naturels tels que $h \leq(g+1) T$ et $k \leq S_{0}$. D'après le lemme de comparaison 8.18 , on a

$$
\begin{aligned}
\max _{|\mathbf{j}|=h}\left|\frac{1}{(\boldsymbol{\tau}+\mathbf{j}) !} \mathcal{D}_{\mathbf{w}}^{\boldsymbol{\tau}+\mathbf{j}} F_{s}(k, k \widetilde{u})\right|_{v_{0}} \leq & d(u, V)\|s\|_{\alpha, v_{0}} \exp \left(\frac{q \overline{33} U}{C_{0}}\right) \max _{\substack{\lambda_{0} \leq D_{0} \\
h \leq 2(g+1) T}}\left|\frac{P_{\lambda_{0}}^{(h)}}{h !}(k)\right|_{v_{0}} \\
& +\max _{|\mathbf{j}|=h}\left|\frac{1}{(\boldsymbol{\tau}+\mathbf{j}) !} \mathcal{D}_{\mathbf{w}}^{\boldsymbol{\tau}+\mathbf{j}} F_{s}(k, k u)\right|_{v_{0}} .
\end{aligned}
$$


Par ailleurs, pour tout $\mathbf{j}$ tel que $|\mathbf{j}|=h$ et tout $k \leq S_{0}$, le couple $(k, \boldsymbol{\tau}+\mathbf{j})$ appartient

à $\Upsilon$. On en déduit que

$$
\begin{aligned}
\max _{|\mathbf{j}|=h}\left|\frac{1}{(\boldsymbol{\tau}+\mathbf{j}) !} \mathcal{D}_{\mathbf{w}}^{\boldsymbol{\tau}+\mathbf{j}} F_{s}(k, k u)\right|_{v_{0}} \leq \alpha^{-1}\|s\|_{\alpha, v_{0}} & =\exp \left(-U\left(g+1+\sqrt{C_{0}}\right)\right)\|s\|_{\alpha, v_{0}} \\
& \leq \exp \left(-U\left(3(g+1) / 4+\sqrt{C_{0}}\right)\right)\|s\|_{\alpha, v_{0}} .
\end{aligned}
$$

L'inégalité (8.14) entraîne alors

$$
\begin{aligned}
\left|\frac{f^{(h)}}{h !}(k)\right|_{v_{0}} \leq & \exp \left(\frac{c^{\prime} U}{C_{0}}\right)\|s\|_{\alpha, v_{0}} \\
& \times\left(d(u, V) \exp \left(\frac{q 33}{C_{0}}\right) \max _{\substack{\lambda_{0} \leq D_{0} \\
h \leq 2(g+1) T}}\left|\frac{P_{\lambda_{0}}^{(h)}}{h !}(k)\right|_{v_{0}}+e^{-U\left(3(g+1) / 4+\sqrt{C_{0}}\right)}\right) .
\end{aligned}
$$

Pour un choix convenable de $c$ et de $c^{\prime}$, les lemmes 8.5 et 8.9 entraînent de plus

$$
\begin{aligned}
2|f|_{R} & \leq c^{T} D_{0}^{\prime} \prod_{i=1}^{n} \exp \left(c D_{i}\left(1+R\left\|u_{i}\right\|_{v_{0}}\right)^{\rho_{i}}\right) \max _{\substack{\lambda_{0} \leq D_{0} \\
|z| \leq R}}\left|\frac{P_{\lambda_{0}}^{\left(\tau_{0}\right)}}{\tau_{0} !}(z)\right|_{v_{0}}\|s\|_{\alpha, v_{0}} \\
& \leq \exp \left(\frac{c^{\prime} U}{C_{0}}\right) \max _{\substack{\lambda_{0} \leq D_{0} \\
|z| \leq R}}\left|\frac{P_{\lambda_{0}}^{\left(\tau_{0}\right)}}{\tau_{0} !}(z)\right|_{v_{0}}\|s\|_{\alpha, v_{0}} .
\end{aligned}
$$

Par ailleurs, si $C_{0}$ est assez grand, on a

$$
5\left(\frac{9(g+1) S}{S_{0}}\right)^{(g+1) T S_{0}} \leq \exp \left((g+1) \log \left(18(g+1) C_{0}^{2}\right) U\right) \leq e^{\sqrt{C_{0}} U}
$$

et $(1 / \mathfrak{e})^{(g+1) T S_{0}} \leq(1 / \mathfrak{e})^{3(g+1) \widetilde{T} S_{0} / 4}=\exp (-3(g+1) U / 4)$. D'après l'inégalité $(8.13)$, on en déduit que

$$
\begin{aligned}
|f(m)|_{v_{0}} \leq & e^{-3(g+1) U / 4} \exp \left(\frac{c^{\prime} U}{C_{0}}\right) \underset{\substack{\lambda_{0} \leq D_{0} \\
h \leq 2(g+1) T \\
|z| \leq R}}{\max }\left|\frac{P_{\lambda_{0}}^{(h)}}{h !}(z)\right|_{v_{0}}\|s\|_{\alpha, v_{0}} \\
& +\exp \left(\sqrt{C_{0}} U\right) \exp \left(\frac{c^{\prime} U}{C_{0}}\right)\|s\|_{\alpha, v_{0}} \\
& \times\left[d(u, V) \max _{\substack{\lambda_{0} \leq D_{0} \\
h \leq 2(g+1) T \\
k \leq S_{0}}}\left|\frac{P_{\lambda_{0}}^{(h)}}{h !}(k)\right|_{v_{0}} \exp \left(\frac{q 33}{C_{0}}\right)+e^{-U\left(3(g+1) / 4+\sqrt{C_{0}}\right)}\right] .
\end{aligned}
$$

Si $C_{0}$ est suffisamment grand, l'hypothèse 2 page 43 entraîne que

$$
d(u, V) \leq \exp \left(-\left(3(g+1) / 4+\frac{c \overline{33}}{C_{0}}+\sqrt{C_{0}}\right) U\right),
$$

et on en déduit que

$$
|f(m)|_{v_{0}} \leq 3 \exp \left(-U\left(3(g+1) / 4-c^{\prime} / C_{0}\right)\right) \times \max _{\substack{\lambda_{0} \leq D_{0} \\ h \leq 2(g+1) T \\|z| \leq R}}\left|\frac{P_{\lambda_{0}}^{(h)}}{h !}(z)\right|_{v_{0}}\|s\|_{\alpha, v_{0}} .
$$


D'après le lemme 8.18 , il existe une constante $c^{\prime \prime}$ telle que

$$
\begin{aligned}
\left|\frac{1}{\tau !} \mathcal{D}_{\mathbf{w}}^{\tau} F_{s}(m, m u)\right|_{v_{0}} & \leq|f(m)|_{v_{0}}+d(u, V) \exp \left(\frac{\Phi_{33} U}{C_{0}}\right)\|s\|_{\alpha, v_{0}} \max _{\substack{|z| \leq R \\
\lambda_{0} \leq D_{0} \\
h \leq 2(g+1) T}}\left|\frac{P_{\lambda_{0}}^{(h)}}{h !}(z)\right|_{v_{0}} \\
& \leq \exp \left(-U\left(3(g+1) / 4-c^{\prime \prime} / C_{0}\right)\right) \times \max _{\substack{|z| \leq R \\
\lambda_{0} \leq D_{0} \\
h \leq 2(g+1) T}}\left|\frac{P_{\lambda_{0}}^{(h)}}{h !}(z)\right|_{v_{0}}\|s\|_{\alpha, v_{0}} .
\end{aligned}
$$

Par ailleurs, quitte à agrandir $c^{\prime \prime}$, le lemme 8.5 entraîne que pour tout $i \in\{1, \ldots, n\}$,

$$
\left|\varphi_{i, \varepsilon_{i}}\left(m u_{i}\right)\right|_{v_{0}}^{-D_{i}} \leq \exp \left(q_{20} D_{i}\left(1+R\left\|u_{i}\right\|_{v_{0}}\right)^{\rho_{i}}\right) \leq e^{c^{\prime \prime} U / C_{0}} .
$$

Si $C_{0}$ est suffisamment grand, on obtient bien le résultat recherché.

8.8.2. Cas ultramétrique. Supposons que la place $v_{0}$ est finie et soit $i \in\{1, \ldots, n\}$. Rappelons que $\left(\varphi_{i, 0}(0), \ldots, \varphi_{i, N_{i}}(0)\right)=(1,0, \ldots, 0)$ et que pour tout $0 \leq j \leq N_{i}$, il existe une suite $\left(a_{j, \mathbf{n}}\right)_{\mathbf{n} \in \mathbb{N} g_{i}}$ d'éléments de $\mathscr{O}_{K_{v_{0}}}$ telle que

$$
\forall \mathbf{z} \in D_{i}\left(0, r_{p_{0}}\right), \quad \varphi_{i, j}(\mathbf{z})=\sum_{\mathbf{n} \in \mathbb{N} g_{i}} \frac{a_{j, \mathbf{n}}}{\mathbf{n} !} \mathbf{z}^{\mathbf{n}} .
$$

Soit $\mathbf{z} \in D_{i}\left(0, r_{p_{0}}\right)$. Comme $a_{0,0}=1$ et que $\left|\mathbf{z}^{\mathbf{n}} / \mathbf{n} !\right|_{v_{0}}<1$ pour tout $\mathbf{n} \neq \mathbf{0}$ (remarquons que $r_{p_{0}}^{|\mathbf{n}|} \leq|\mathbf{n} !|_{v_{0}}$ pour tout $\left.\mathbf{n} \in \mathbb{N}^{g}\right)$, on a $\left|\varphi_{i, 0}(\mathbf{z})\right|_{v_{0}}=1$. De la même façon on remarque que $\left|\varphi_{i, j}(\mathbf{z})\right|_{v_{0}}<1$ si $j \neq 0$. En particulier $\max _{0 \leq j \leq N_{i}}\left|\varphi_{i, j}(z)\right|_{v_{0}}=\left|\varphi_{i, 0}(z)\right|_{v_{0}}$ pour tout $z \in D_{i}\left(0, r_{p_{0}}\right)$ et $\left|\varphi_{i, \varepsilon_{i}}\left(m u_{i}\right)\right|_{v_{0}}=\left|\varphi_{i, 0}\left(m u_{i}\right)\right|_{v_{0}}=1$. On a ainsi

$$
\left|\prod_{i=1}^{n} \varphi_{i, \varepsilon_{i}}\left(m u_{i}\right)^{-D_{i}} \frac{1}{\boldsymbol{\tau} !} \mathcal{D}_{\mathbf{w}}^{\boldsymbol{\tau}} F_{s}(m, m u)\right|_{v_{0}}=\left|\frac{1}{\boldsymbol{\tau} !} \mathcal{D}_{\mathbf{w}}^{\boldsymbol{\tau}} F_{s}(m, m u)\right|_{v_{0}} .
$$

Nous allons maintenant reprendre les arguments des lemmes 8.8 et 8.9 afin d'établir un lemme de comparaison analogue au lemme 8.18. Pour tous $\mathbf{z}_{1}, \mathbf{z}_{1}^{\prime} \in D\left(0, r_{p_{0}}\right)$ et tout $\mathbf{n} \in \mathbb{N}^{g} \backslash\{0\}$, on a

$$
\left|\mathbf{z}_{1}^{\mathbf{n}}-\mathbf{z}_{1}^{\prime \mathbf{n}}\right|_{v_{0}} \leq\left\|\mathbf{z}_{1}-\mathbf{z}_{1}^{\prime}\right\|_{v_{0}} \max \left\{\left\|\mathbf{z}_{1}\right\|_{v_{0}},\left\|\mathbf{z}_{1}^{\prime}\right\|_{v_{0}}\right\}^{|\mathbf{n}|-1},
$$

d'où

$$
\left|\frac{\mathbf{z}_{1}^{\mathbf{n}}}{\mathbf{n} !}-\frac{\mathbf{z}_{1}^{\prime \mathbf{n}}}{\mathbf{n} !}\right|_{v_{0}} \leq \frac{\left|\mathbf{z}_{1}^{\mathbf{n}}-\mathbf{z}_{1}^{\prime \mathbf{n}}\right|_{v_{0}}}{r_{p_{0}}^{|\mathbf{n}|}} \leq\left\|\mathbf{z}_{1}-\mathbf{z}_{1}^{\prime}\right\|_{v_{0}} r_{p_{0}}^{-1} .
$$

La fonction $F_{s}$ s'écrit $F_{s}=\sum_{\boldsymbol{\lambda} \in \Lambda} p_{\boldsymbol{\lambda}} F_{s_{\lambda}}$, où

$$
\Lambda=\left\{\boldsymbol{\lambda}=\left(\lambda_{0},\left(\boldsymbol{\lambda}_{i}\right)_{1 \leq i \leq n}\right) \in \mathbb{N} \times \prod_{i=1}^{n} \mathbb{N}^{N_{i}+1}\left|\lambda_{0} \leq D_{0}, \boldsymbol{\lambda}_{i}=\left(\lambda_{i, j}\right)_{0 \leq j \leq N_{i}},\right| \boldsymbol{\lambda}_{i} \mid=D_{i}\right\}
$$

et $F_{s_{\boldsymbol{\lambda}}}=P_{\lambda_{0}} \prod_{i=1}^{n} \prod_{j=0}^{N_{i}} \varphi_{i, j}^{\lambda_{i, j}}$ pour tout $\boldsymbol{\lambda} \in \Lambda$. Soit $\boldsymbol{\sigma}=\left(\sigma_{0}, \boldsymbol{\sigma}_{1}\right) \in \mathbb{N} \times \mathbb{N}^{g}$. Par choix de la base $\mathbf{e}$ (définie à la page 17 ), pour tout $\boldsymbol{\lambda} \in \Lambda$, il existe une suite $\left(a_{\mathbf{n}, \boldsymbol{\lambda}}\right)_{\mathbf{n} \in \mathbb{N}^{g}}$ d'éléments de $\mathscr{O}_{K_{v_{0}}}$ telle que pour tout $k \in \mathbb{N}$, on ait

$$
\frac{1}{\boldsymbol{\sigma} !} \mathcal{D}_{\left(e_{0}, \mathbf{e}\right)}^{\boldsymbol{\sigma}} F_{s_{\boldsymbol{\lambda}}}\left(k, k \mathbf{z}_{1}\right)-\frac{1}{\boldsymbol{\sigma} !} \mathcal{D}_{\left(e_{0}, \mathbf{e}\right)}^{\boldsymbol{\sigma}} F_{s_{\boldsymbol{\lambda}}}\left(k, k \mathbf{z}_{1}^{\prime}\right)=\frac{P_{\lambda_{0}}^{\left(\sigma_{0}\right)}}{\sigma_{0} !}(k) \sum_{\mathbf{n}} \frac{a_{\mathbf{n}, \boldsymbol{\lambda}}}{\left(\mathbf{n}-\boldsymbol{\sigma}_{1}\right) !}\left(\mathbf{z}_{1}^{\mathbf{n}-\boldsymbol{\sigma}_{1}}-\mathbf{z}_{1}^{\prime \mathbf{n}-\boldsymbol{\sigma}_{1}}\right),
$$


où la somme est prise sur l'ensemble des $\mathbf{n}=\left(n_{1}, \ldots, n_{g}\right) \in \mathbb{N}^{g}$ tels que $n_{j} \geq \sigma_{1, j}$ pour tout $j$ (en posant $\boldsymbol{\sigma}_{1}=\left(\sigma_{1,1}, \ldots, \sigma_{1, g}\right)$ ). En raisonnant comme dans la démonstration du lemme 8.8. on montre que pour tout $\mathbf{h}=\left(h_{0}, \mathbf{h}_{1}\right) \in \mathbb{N} \times \mathbb{N}^{g-t}$, la quantité

$$
\left|\frac{1}{\mathbf{h} !} \mathcal{D}_{\mathbf{w}}^{\mathbf{h}} F_{s_{\boldsymbol{\lambda}}}\left(k, k \mathbf{z}_{1}\right)-\frac{1}{\mathbf{h} !} \mathcal{D}_{\mathbf{w}}^{\mathbf{h}} F_{s_{\boldsymbol{\lambda}}}\left(k, k \mathbf{z}_{1}^{\prime}\right)\right|_{v_{0}}
$$

est majorée par

$$
\max _{\boldsymbol{\sigma}}\left|\frac{1}{\boldsymbol{\sigma} !} \mathcal{D}_{\left(e_{0}, \mathbf{e}\right)}^{\boldsymbol{\sigma}} F_{s_{\boldsymbol{\lambda}}}\left(k, k \mathbf{z}_{1}\right)-\frac{1}{\boldsymbol{\sigma} !} \mathcal{D}_{\left(e_{0}, \mathbf{e}\right)}^{\boldsymbol{\sigma}} F_{s_{\boldsymbol{\lambda}}}\left(k, k \mathbf{z}_{1}^{\prime}\right)\right|_{v_{0}},
$$

où le maximum est pris sur l'ensemble des couples $\boldsymbol{\sigma}=\left(h_{0}, \boldsymbol{\sigma}_{1}\right) \in \mathbb{N} \times \mathbb{N}^{g}$ tels que $\left|\boldsymbol{\sigma}_{1}\right|=\left|\mathbf{h}_{1}\right|$. On en déduit le lemme de comparaison suivant.

Lemme 8.20. Soient $\mathbf{z}_{1}, \mathbf{z}_{1}^{\prime} \in D\left(0, r_{p_{0}}\right)$. Alors pour tout couple $\mathbf{h}=\left(h_{0}, \mathbf{h}_{1}\right) \in \mathbb{N} \times \mathbb{N}^{g-t}$ et tout $k \in \mathbb{N}$, on $a$

$$
\left|\frac{1}{\mathbf{h} !} \mathcal{D}_{\mathbf{w}}^{\mathbf{h}} F_{s}\left(k, k \mathbf{z}_{1}\right)-\frac{1}{\mathbf{h} !} \mathcal{D}_{\mathbf{w}}^{\mathbf{h}} F_{s}\left(k, k \mathbf{z}_{1}^{\prime}\right)\right|_{v_{0}} \leq r_{p_{0}}^{-1} \max _{\lambda_{0} \leq D_{0}}\left|\frac{P_{\lambda_{0}}^{\left(h_{0}\right)}}{h_{0} !}(k)\right|_{v_{0}}\|s\|\left\|_{E, v_{0}}\right\| \mathbf{z}_{1}-\mathbf{z}_{1}^{\prime} \|_{v_{0}} .
$$

Rappelons que $\widetilde{u}$ désigne un vecteur de $V \otimes_{K} \mathbb{C}_{v_{0}}$ tel que $\|u-\widetilde{u}\|_{v_{0}}=d(u, V)$ et que la base $\mathbf{w}$ de $W \otimes_{K} \mathbb{C}_{v_{0}}$ est formée de vecteurs de norme 1 (voir page 38). D'après la proposition 2.5 page 11 , nous pouvons supposer que cette base vérifie

$$
\forall\left(x_{1}, \ldots, x_{g-t}\right) \in \mathbb{C}_{v_{0}}^{g-t}, \quad\left\|\sum_{j=1}^{g-t} x_{j} w_{j}\right\|_{v_{0}}>r_{p_{0}} \max _{1 \leq j \leq g-t}\left|x_{j}\right|_{v_{0}} .
$$

Nous avons maintenant tous les outils nécessaires à l'extrapolation dans le cas ultramétrique, qui conduit au résultat suivant. Rappelons que l'on a supposé que $\|u\|_{v_{0}}<r_{p_{0}}$ et que $\mathfrak{R}$ est un nombre réel de l'intervalle $] 1, r_{p_{0}} /\|u\|_{v_{0}}[$.

Proposition 8.21. Il existe une constante $\overline{955} \geq 1$ telle que si $C_{0} \geq q 5$, alors

$$
\begin{aligned}
& \left|\prod_{i=1}^{n} \varphi_{i, \varepsilon_{i}}\left(m u_{i}\right)^{-D_{i}} \frac{1}{\boldsymbol{\tau} !} \mathcal{D}_{\mathbf{w}}^{\boldsymbol{\tau}} F_{s}(m, m u)\right|_{v_{0}} \\
& \quad \leq e^{-U}\|s\|_{v_{0}, \alpha} \times \max \left\{\left.\left|\frac{P_{\lambda_{0}}^{(h)}}{h !}(z)\right|_{v_{0}}\left|h \leq 2(g+1) T, \lambda_{0} \leq D_{0},\right| z\right|_{v_{0}} \leq \mathfrak{R}\right\} .
\end{aligned}
$$

Démonstration. D'après 8.15, on a

$$
\left|\prod_{i=1}^{n} \varphi_{i, \varepsilon_{i}}\left(m u_{i}\right)^{-D_{i}} \frac{1}{\boldsymbol{\tau} !} \mathcal{D}_{\mathbf{w}}^{\boldsymbol{\tau}} F_{s}(m, m u)\right|_{v_{0}}=\left|\frac{1}{\boldsymbol{\tau} !} \mathcal{D}_{\mathbf{w}}^{\boldsymbol{\tau}} F_{s}(m, m u)\right|_{v_{0}} .
$$

Considérons la fonction

$$
f:\left\{\left.\nu \in \mathbb{C}_{v_{0}}|| \nu\right|_{v_{0}}<r_{p_{0}} /\|u\|_{v_{0}}\right\} \ni \nu \mapsto \frac{1}{\tau !} \mathcal{D}_{\mathbf{w}}^{\boldsymbol{\tau}} F_{s}(\nu, \nu \widetilde{u}) .
$$

Remarquons que $d(u, V)=\|u-\widetilde{u}\|_{v_{0}}=\inf \left\{\|u-\mathbf{z}\|_{v_{0}} \mid \mathbf{z} \in V \otimes_{K} \mathbb{C}_{v_{0}}\right\} \leq\|u\|_{v_{0}}$. Si $d(u, V)=\|u\|_{v_{0}}$, on peut poser $\widetilde{u}=0$. Sinon, on a $d(u, V)<\|u\|_{v_{0}}$, et donc $\|\widetilde{u}\|_{v_{0}}=\|u\|_{v_{0}}$. Dans tous les cas, on en déduit que $f$ est analytique sur $\left\{\left.\nu \in \mathbb{C}_{v_{0}}|| \nu\right|_{v_{0}}<r_{p_{0}} /\|u\|_{v_{0}}\right\}$. 
En appliquant le lemme d'interpolation ultramétrique du paragraphe 6 (corollaire 6.3 page 33 à $f$ avec $R=\Re>1, S_{1}=S_{0}$ et $T_{1}=(g+1) T$, on a

$$
|f(m)|_{v_{0}} \leq \max \left\{\Re^{-(g+1) T S_{0}}|f|_{v_{0}, \Re}, \gamma \max _{\substack{h, k \in \mathbb{N} \\ h<(g+1) T, k<S_{0}}}\left|\frac{f^{(h)}(k)}{h !}\right|_{v_{0}}\right\}
$$

où

$$
\gamma=p_{0}^{((g+1) T-1)\left[\log \left(S_{0}-1\right) / \log p_{0}\right]} \quad \text { et } \quad|f|_{v_{0}, \mathfrak{R}}=\sup \left\{\left.|f(z)|_{v_{0}}\left|z \in \mathbb{C}_{v_{0}},\right| z\right|_{v_{0}} \leq \mathfrak{R}\right\} .
$$

Notons $\widetilde{\mathbf{x}}=\left(1, \widetilde{x}_{1}, \ldots, \widetilde{x}_{g-t}\right)$ les coordonnées de $(1, \widetilde{u})$ dans la base $\mathbf{w}$ :

$$
(1, \widetilde{u})=e_{0}+\sum_{j=1}^{n} \widetilde{x}_{j} w_{j}
$$

L'hypothèse faite sur $\mathbf{w}$ entraîne $\max _{1 \leq j \leq g-t}\left|\widetilde{x}_{j}\right|_{v_{0}}<r_{p_{0}}^{-1}\|\widetilde{u}\|_{v_{0}}<1$. Pour tous entiers $h, k \geq 0$, on a

$$
\frac{1}{h !} f^{(h)}(k)=\sum_{\substack{|\mathbf{j}|=h \\
\mathbf{j} \in \mathbb{N}^{g+1-t}}}\left(\begin{array}{c}
\boldsymbol{\tau}+\mathbf{j} \\
\mathbf{j}
\end{array}\right) \widetilde{\mathbf{x}}^{\mathbf{j}} \frac{1}{(\boldsymbol{\tau}+\mathbf{j}) !} \mathcal{D}_{\mathbf{w}}^{\boldsymbol{\tau}+\mathbf{j}} F_{s}(k, k \widetilde{u}),
$$

où $\widetilde{\mathbf{x}}^{\mathbf{j}}=\widetilde{x}_{1}^{j_{1}} \cdots \widetilde{x}_{g-t}^{j_{g-t}}$. On en déduit que pour tout $k \leq S_{0}$ et tout $h \leq(g+1) T$,

$$
\begin{aligned}
\left|\frac{1}{h !} f^{(h)}(k)\right|_{v_{0}} & \leq \max _{|\mathbf{j}|=h}\left|\frac{1}{(\boldsymbol{\tau}+\mathbf{j}) !} \mathcal{D}_{\mathbf{w}}^{\boldsymbol{\tau}+\mathbf{j}} F_{s}(k, k \widetilde{u})\right|_{v_{0}} \\
& \leq \max \left\{\max _{|\mathbf{j}|=h\left|\frac{1}{(\boldsymbol{\tau}+\mathbf{j}) !} \mathcal{D}_{\mathbf{w}}^{\boldsymbol{\tau}+\mathbf{j}} F_{s}(k, k \widetilde{u})-\frac{1}{(\boldsymbol{\tau}+\mathbf{j}) !} \mathcal{D}_{\mathbf{w}}^{\boldsymbol{\tau}+\mathbf{j}} F_{s}(k, k u)\right|_{v_{0}},}^{\left.\max _{|\mathbf{j}|=h}\left|\frac{1}{(\boldsymbol{\tau}+\mathbf{j}) !} \mathcal{D}_{\mathbf{w}}^{\boldsymbol{\tau}+\mathbf{j}} F_{s}(k, k u)\right|_{v_{0}}\right\} .}\right.
\end{aligned}
$$

En appliquant le lemme 8.20 et en remarquant que $(k, \boldsymbol{\tau}+\mathbf{j}) \in \boldsymbol{\Upsilon}$ pour tout $|\mathbf{j}|=h$, on a

$$
\left|\frac{1}{h !} f^{(h)}(k)\right|_{v_{0}} \leq\|s\|_{v_{0}, \alpha} \max \left\{\max _{\lambda_{0} \leq D_{0}}\left|\frac{P_{\lambda_{0}}^{\left(h_{0}\right)}}{h_{0} !}(k)\right|_{v_{0}} r_{p_{0}}^{-1} d(u, V), \alpha^{-1}\right\} .
$$

Comme $\mathfrak{R}<r_{p_{0}} /\|u\|_{v_{0}}$, le lemme 8.9 entraîne par ailleurs que

$$
|f|_{v_{0}, \Re} \leq \max _{\substack{|z|_{v_{0}} \leq \Re \\ \lambda_{0} \leq D_{0}}}\left|\frac{P_{\lambda_{0}}^{\left(\tau_{0}\right)}}{\tau_{0} !}(z)\right|_{v_{0}}\|s\|_{v_{0}, \alpha}
$$

D'après le lemme 8.6(7), le réel $\gamma$ de l'inégalité 8.16) est majoré par

$$
\exp \left((g+1) T \log S_{0}\right) \leq \exp \left((g+1) T S_{0} \log \mathfrak{R}\right) \leq \exp ((g+1) U) .
$$

De plus, on a

$$
\mathfrak{R}^{-(g+1) T S_{0}} \leq \mathfrak{R}^{-(g+1) \widetilde{T} S_{0} / 2}=\exp (-(g+1) U / 2) \leq e^{-U} .
$$

D'après l'inégalité 8.16 et les remarques précédentes, la valeur absolue $|f(m)|_{v_{0}}$ est majorée par le produit de $\|s\|_{v_{0}}$ avec 


$$
\max \left\{e^{-U} \max _{\substack{|z|_{v_{0}} \leq \mathfrak{R} \\ \lambda_{0} \leq D_{0}}}\left|\frac{P_{\lambda_{0}}^{\left(\tau_{0}\right)}}{\tau_{0} !}(z)\right|_{v_{0}}, e^{(g+1) U} \max _{\substack{h \leq(g+1) T \\ \lambda_{0} \leq D_{0} \\ k \leq S_{0}}}\left|\frac{P_{\lambda_{0}}^{\left(h_{0}\right)}}{h_{0} !}(k)\right|_{v_{0}} \max \left\{r_{p_{0}}^{-1} d(u, V), \alpha^{-1}\right\}\right\} .
$$

De plus,

$$
\alpha^{-1}=\exp \left(-U\left(g+1+\sqrt{C_{0}}\right)\right) \leq \exp (-(g+1) U-U)
$$

et si $C_{0}$ est suffisamment grand, on a

$$
r_{p_{0}}^{-1} d(u, V) \leq r_{p_{0}}^{-1} \exp \left(-2 \sqrt{C_{0}} U\right) \leq \exp (-(g+1) U-U) .
$$

On obtient alors

$$
|f(m)|_{v_{0}} \leq e^{-U}\|s\|_{v_{0}, \alpha} \max \left\{\left.\left|\frac{P_{\lambda_{0}}^{(h)}}{h !}(z)\right|_{v_{0}}\left|h \leq 2(g+1) T, \lambda_{0} \leq D_{0},\right| z\right|_{v_{0}} \leq \mathfrak{R}\right\} .
$$

On conclut en appliquant une nouvelle fois le lemme 8.20

8.9. Encadrement de la hauteur du jet. Rappelons que $D=[K: \mathbb{Q}] /\left[K_{v_{0}}: \mathbb{Q}_{v_{0}}\right]$.

\subsubsection{Minoration}

Lemme 8.22. Il existe une constante व6 telle que la hauteur de $\mathcal{J} \in S^{\ell}\left(W^{\vee}\right) \otimes_{K} K^{\prime}$ vérifie

$$
h(\mathcal{J}) \geq-\frac{U}{{ }_{36}} \frac{U}{C_{0} D} .
$$

Démonstration. Comme $\mathcal{J} \in S^{\ell}\left(W^{\vee}\right) \otimes_{K} K^{\prime}$ est non nul, on a $h(\mathcal{J}) \geq-\widehat{\mu}_{\max }\left(S^{\ell}\left(W^{\vee}\right)\right)$. D'après la proposition 2.13 de la page 14 , on en déduit que

$$
h(\mathcal{J}) \geq-\widehat{\mu}_{\max }\left(S^{\ell}\left(W^{\vee}\right)\right) \geq-\ell \widehat{\mu}_{\max }\left(W^{\vee}\right)-2 \ell \log g .
$$

Par ailleurs, on a

$$
\begin{aligned}
h(W)=-\widehat{\operatorname{deg}}_{\mathrm{n}}(W) & =-\sum_{i=1}^{g-t} \widehat{\mu}_{i}(W)=\widehat{\mu}_{\max }\left(W^{\vee}\right)+\sum_{i=2}^{g-t} \widehat{\mu}_{i}\left(W^{\vee}\right) \\
& \geq \widehat{\mu}_{\max }\left(W^{\vee}\right)-(g-t-1) \widehat{\mu}_{\max }\left(t_{G_{0} \times G}\right),
\end{aligned}
$$

car chacune des pentes successives $\widehat{\mu}_{i}\left(W^{\vee}\right)$ vérifie

$$
\widehat{\mu}_{i}\left(W^{\vee}\right)=-\widehat{\mu}_{\operatorname{dim} W+1-i}(W) \geq-\widehat{\mu}_{\max }(W) \geq-\widehat{\mu}_{\max }\left(t_{G_{0} \times G}\right) .
$$

Comme $\ell \leq(g+1) T$, on en déduit que $h(\mathcal{J}) \geq-{ }_{\overline{36}} T \max \{1, h(W)\}$, et l'on conclut en utilisant la définition de $T$.

\subsubsection{Majoration dans le cas archimédien}

Proposition 8.23. Supposons que la place $v_{0}$ est archimédienne et que le réel $C_{0}$ est supérieur à $\max \left\{q_{32}^{2}, q_{34}\right\}$. Alors

$$
h(\mathcal{J}) \leq \frac{2 q_{29} U}{D \sqrt{C_{0}}}-\frac{U}{2 D}+\aleph\left(\left(P_{\lambda_{0}}\right)_{\lambda_{0}}\right)
$$


où $\left(\left(P_{\lambda_{0}}\right)_{\lambda_{0}}\right)$ désigne la quantité

$$
\sum_{v \in \Sigma_{K}} \frac{\left[K_{v}: \mathbb{Q}_{v}\right]}{[K: \mathbb{Q}]} \log \max _{\substack{k \in \mathbb{N}, k \leq(g+1) S \\ \lambda_{0} \leq D_{0} \\ h \leq 2(g+1) T}}\left|\frac{P_{\lambda_{0}}^{(h)}}{h !}(k)\right|_{v}+\frac{1}{D} \log \max _{\substack{|z| \leq 2(g+1) S \mathfrak{e} \\ \lambda_{0} \leq D_{0} \\ h \leq 2(g+1) T}}\left|\frac{P_{\lambda_{0}}^{(h)}}{h !}(z)\right|_{v_{0}} .
$$

Démonstration. D'après la définition de $\|\mathcal{J}\|_{v}$ et la proposition 8.19 , il existe une constante $c \geq 1$ ne dépendant que de $g$ telle que

$$
\begin{aligned}
\sum_{v \in \Sigma_{K^{\prime}}, v \mid v_{0}} \frac{\left[K_{v}^{\prime}: \mathbb{Q}_{v}\right]}{\left[K^{\prime}: \mathbb{Q}\right]} \log \|\mathcal{J}\|_{v} \\
\quad \leq \sum_{v \in \Sigma_{K^{\prime}}, v \mid v_{0}} \frac{\left[K_{v}^{\prime}: \mathbb{Q} v\right]}{\left[K^{\prime}: \mathbb{Q}\right]}\left(-U+\log \|s\|_{\alpha, v}+\log \max _{\substack{|z| \leq 2(g+1) S \mathfrak{e} \\
\lambda_{0} \leq D_{0} \\
h \leq 2(g+1) T}}\left|\frac{P_{\lambda_{0}}^{(h)}}{h !}(z)\right|_{v_{0}}+c T\right) \\
\leq \sum_{v \in \Sigma_{K^{\prime}}, v \mid v_{0}} \frac{\left[K_{v}^{\prime}: \mathbb{Q}_{v}\right]}{\left[K^{\prime}: \mathbb{Q}\right]} \log \|s\|_{\alpha, v}+\frac{1}{D}\left(-\frac{U}{2}+\log _{\substack{|z| \leq 2(g+1) S \mathfrak{e} \\
\lambda_{0} \leq D_{0} \\
h \leq 2(g+1) T}}\left|\frac{P_{\lambda_{0}}^{(h)}}{h !}(z)\right|_{v_{0}}\right) .
\end{aligned}
$$

En combinant cette inégalité avec la proposition 8.17, on obtient

$$
\begin{gathered}
h(\mathcal{J}) \leq h_{\alpha}(s)+{ }_{32} \frac{U}{C_{0} D}-\frac{U}{2 D}+\frac{1}{D} \log \max _{\substack{|z| \leq 2(g+1) S \mathfrak{e} \\
\lambda_{0} \leq D_{0} \\
h \leq 2(g+1) T}}\left|\frac{P_{\lambda_{0}}^{(h)}}{h !}(z)\right|_{v_{0}} \\
+\sum_{v \in \Sigma_{K}, v \neq v_{0}} \frac{\left[K_{v}: \mathbb{Q} v\right]}{[K: \mathbb{Q}]} \log \max _{\substack{\lambda_{0} \leq D_{0} \\
h \leq(g+1) T}}\left|\frac{P_{\lambda_{0}}^{(h)}}{h !}(m)\right|_{v}
\end{gathered}
$$

D'après la proposition 8.11 , on a par ailleurs

$$
h_{\alpha}(s) \leq \frac{q \overline{29}}{D \sqrt{C_{0}}} U+\frac{1}{D} \max _{\substack{\lambda_{0} \leq D_{0} \\ k \in \mathbb{N}, k \leq S_{0} \\ h \leq 2(g+1) T}} \log \left|\frac{P_{\lambda_{0}}^{(h)}}{h !}(k)\right|_{v_{0}},
$$

ce qui achève la preuve.

\subsubsection{Majoration dans le cas ultramétrique}

Proposition 8.24. Supposons que la place $v_{0}$ est finie et que $C_{0} \geq \max \left\{q_{32}^{2}, q 35\right\}$. Alors

$$
h(\mathcal{J}) \leq-\frac{U}{D}+\frac{2 q_{29} U}{D \sqrt{C_{0}}}+\aleph\left(\left(P_{\lambda_{0}}\right)_{\lambda_{0}}\right)
$$

où

$$
\begin{aligned}
\aleph\left(\left(P_{\lambda_{0}}\right)_{\lambda_{0}}\right)= & \sum_{v \in \Sigma_{K}} \frac{\left[K_{v}: \mathbb{Q} v\right]}{[K: \mathbb{Q}]} \log \max _{\substack{k \in \mathbb{N}, k \leq(g+1) S \\
\lambda_{0} \leq D_{0} \\
h \leq 2(g+1) T}}\left|\frac{P_{\lambda_{0}}^{(h)}}{h !}(k)\right|_{v} \\
& +\frac{1}{D} \log \max \left\{\left|\frac{\left.\left.\left.P_{\lambda_{0}}^{(h)}(z)\right|_{v_{0}}\left|h \leq 2(g+1) T, \lambda_{0} \leq D_{0},\right| z\right|_{v_{0}} \leq \mathfrak{R}\right\} .}{h !}\right|\right.
\end{aligned}
$$


Démonstration. D'après les propositions 8.17 et 8.21, on a

$$
\begin{aligned}
h(\mathcal{J}) \leq & h_{\alpha}(s)-\frac{U}{D} \\
& +\frac{1}{D} \log \max \left\{\left.\left|\frac{P_{\lambda_{0}}^{(h)}}{h !}(z)\right|_{v_{0}}\left|h \leq 2(g+1) T, \lambda_{0} \leq D_{0},\right| z\right|_{v_{0}} \leq \mathfrak{R}\right\} \\
& +\sum_{v \neq v_{0}} \frac{\left[K_{v}: \mathbb{Q} v\right]}{[K: \mathbb{Q}]} \log \max _{\substack{h \leq(g+1) T \\
\lambda_{0} \leq D_{0}}}\left|\frac{P_{\lambda_{0}}^{(h)}}{h !}(m)\right|_{v}+{ }^{{ }_{32} \frac{U}{D C_{0}}} .
\end{aligned}
$$

D'après la proposition 8.11, la hauteur $h_{\alpha}(s)$ est majorée par

$$
\text { q29 } \frac{U}{D \sqrt{C_{0}}}+\frac{1}{D} \max _{\substack{\lambda_{0} \leq D_{0} \\ k \in \mathbb{N}, k \leq S_{0} \\ h \leq 2(g+1) T}} \log \left|\frac{P_{\lambda_{0}}^{(h)}}{h !}(k)\right|_{v_{0}} .
$$

On en déduit immédiatement la majoration voulue.

8.10. Poids de la droite affine. L'objet de ce paragraphe est de fixer le choix de la famille de polynômes $\left(P_{\lambda_{0}}\right)_{\lambda}$ et de majorer les quantités $\aleph\left(\left(P_{\lambda_{0}}\right)_{\lambda_{0}}\right)$ qui apparaissent dans les propositions 8.23 et 8.24 . La majoration de $\aleph\left(\left(P_{\lambda_{0}}\right)_{\lambda_{0}}\right)$ a une influence directe sur la minoration de $d(u, V)$ obtenue; comme l'a remarqué Fel'dman [17, il existe des familles de polynômes qui conviennent mieux que la base canonique $\left(X^{\lambda_{0}}\right)_{\lambda_{0} \leq D_{0}}$ de $K[X]_{\leq D_{0}}$. Nous utiliserons une variante des polynômes de Fel'dman, construite par Matveev [31. Considérons les polynômes binomiaux

$$
\Delta_{0}(X):=1, \quad \Delta_{k}(X):=\frac{X(X+1) \cdots(X+k-1)}{k !}, \quad k \in \mathbb{N} \backslash\{0\} .
$$

DÉFInItion 8.25. Étant donnés deux entiers naturels $a$ et $b$, le polynôme de Matveev $\delta_{b}(X ; a)$ est $\Delta_{b}(X)^{q} \Delta_{r}(X)$, où $q$ et $r$ désignent respectivement le quotient et le reste de la division euclidienne de $a$ par $b$.

Rappelons que l'on a noté $D=[K: \mathbb{Q}] /\left[K_{v_{0}}: \mathbb{Q}_{v_{0}}\right]$.

Proposition 8.26 ([21, lemme 3.10]). Soit $b \in \mathbb{N} \backslash\{0\}$. On pose $\mathfrak{r}=2(g+1) \mathfrak{e} S$ si $v_{0}$ est archimédienne, et $\mathfrak{r}=\mathfrak{R}$ si $v_{0}$ est une place finie. Il existe une constante absolue q37 $\geq 1$ vérifiant la propriété suivante. Si $P_{\lambda_{0}}=\delta_{b}\left(X ; \lambda_{0}\right)$ pour tout $\lambda_{0} \leq D_{0}$, alors la quantité $\aleph\left(\left(P_{\lambda_{0}}\right)_{\lambda_{0}}\right)$ égale $\grave{a}$

$$
\sum_{v \in \Sigma_{K}} \frac{\left[K_{v}: \mathbb{Q}_{v}\right]}{[K: \mathbb{Q}]} \log \max _{\substack{k \in \mathbb{N}, k \leq(g+1) S \\ \lambda_{0} \leq D_{0} \\ h \leq 2(g+1) T}}\left|\frac{P_{\lambda_{0}}^{(h)}}{h !}(k)\right|_{v}+\frac{1}{D} \log \max _{\substack{|z|_{v_{0}} \leq \mathfrak{r} \\ \lambda_{0} \leq D_{0} \\ h \leq 2(g+1) T}}\left|\frac{P_{\lambda_{0}}^{(h)}}{h !}(z)\right|_{v_{0}}
$$

est majorée par

$$
\text { q37 }\left(D_{0} \log \left(e+\frac{S}{b}\right)+b T+\frac{D_{0}}{D} \log \left(1+\frac{\mathfrak{e} S}{b}\right)\right)
$$

si $v_{0}$ est archimédienne, et par

$$
\text { q37 }\left(D_{0} \log \left(e+\frac{S}{b}\right)+b T+\frac{D_{0}}{D} \log (\Re)\right) .
$$

si $v_{0}$ est une place finie. 
Remarque 8.27. Le choix de la base canonique de $K[X]_{\lambda_{0} \leq D_{0}}$ pour la famille $\left(P_{\lambda_{0}}\right)_{\lambda_{0}}$ conduit à la majoration

$$
\aleph\left(\left(X^{\lambda_{0}}\right)_{\lambda_{0}}\right) \leq q_{37} D_{0}\left(\log S+\frac{\log \mathfrak{e}}{D}\right),
$$

qui est insuffisante pour démontrer les théorèmes 4.3 et 4.4 .

8.11. Conclusion. Nous allons maintenant combiner les estimations des paragraphes 8.9 et 8.10 pour aboutir à une contradiction.

8.11.1. Cas archimédien. Supposons que la place $v_{0}$ est archimédienne et que le nombre réel $C_{0}$ est supérieur à $\max \left\{q_{21}, q_{22}, q_{32}^{2}, q_{34}\right\}$. Sous l'hypothèse 2 , les propositions 8.22 et 8.23 entraînent

$$
-{ }^{36} \frac{U}{C_{0} D} \leq h(\mathcal{J}) \leq \frac{2 q 29}{D \sqrt{C_{0}}} U-\frac{U}{2 D}+\aleph\left(\left(P_{\lambda_{0}}\right)_{\lambda_{0}}\right),
$$

d'où

$$
U \leq \frac{2[\overline{36}+4 q[29}{\sqrt{C_{0}}} U+D \aleph\left(\left(P_{\lambda_{0}}\right)_{\lambda_{0}}\right) .
$$

On pose $b=\left[\frac{S_{0} \log \mathfrak{e}}{C_{0} D}\right]$ et $P_{\lambda_{0}}=\delta_{b}\left(X ; \lambda_{0}\right)$ pour tout entier naturel $\lambda_{0} \leq D_{0}$. D'après le lemme 8.5 et la proposition 8.26 on a les majorations

$$
\begin{aligned}
\aleph\left(\left(P_{\lambda_{0}}\right)_{\lambda_{0}}\right) & \leq q \underline{37}\left(D_{0} \log \left(e+\frac{S}{b}\right)+b T+\frac{D_{0}}{D} \log \left(1+\frac{\mathfrak{e} S}{b}\right)\right) \\
& \leq q \frac{27}{37}\left(D_{0} \log \left(e+\frac{2 S C_{0} D}{S_{0} \log \mathfrak{e}}\right)+\frac{1}{C_{0} D} T S_{0} \log \mathfrak{e}+\frac{D_{0}}{D} \log \left(1+\frac{2 \mathfrak{e} S C_{0} D}{S_{0} \log \mathfrak{e}}\right)\right) \\
& \leq 3 \frac{D_{0} A_{0}}{D}+q \frac{U}{C_{0} D} \leq 4 q_{37} \frac{U}{C_{0} D} .
\end{aligned}
$$

On obtient ainsi

$$
U \leq \frac{2 q[36+4 q 29+4 q[37}{\sqrt{C_{0}}} U
$$

On a donc une contradiction dès que

$$
C_{0}>\max \left\{q_{21}, q_{22}, q_{32}^{2}, q_{34},\left(2 q_{36}+4 q_{29}+4 q_{37}\right)^{2}\right\} .
$$

Dans ce cas, on en déduit que l'hypothèse 2 ne peut pas être vérifiée et donc que $d(u, V)>$ $\exp \left(-2 \sqrt{C_{0}} U\right)$. Pour conclure, il suffit de comparer $U$ avec la quantité $U_{2}$ du théorème 4.3. On a

$$
\begin{aligned}
U \leq & 8^{\frac{4 g+1}{t}} C_{0}^{2+\frac{1}{t}(4 g+3)} \mathfrak{a} \log \mathfrak{e}\left(\frac{D}{\log \mathfrak{e}} \log \left(e+\frac{D}{\log \mathfrak{e}}\right)+1\right)^{1 / t} \\
& \times \prod_{i=1}^{n}\left(1+\frac{D \max _{k \leq(g+1) C_{0}^{3} \mathfrak{a}} h\left(k \mathbf{p}_{i}\right)+\left(\mathfrak{e} \mathfrak{a}\left\|u_{i}\right\|_{v_{0}}\right)^{\rho_{i}}}{\mathfrak{a} \log \mathfrak{e}}\right)^{g_{i} / t} .
\end{aligned}
$$

Par ailleurs, il existe une constante 438 telle que $\frac{D}{\log \mathfrak{e}} \log \left(e+\frac{D}{\log \mathfrak{e}}\right) \leq 93 \frac{D}{\log \mathfrak{e}} \log \left(\frac{D}{\log \mathfrak{e}}\right)$. Le théorème 4.3 est donc démontré, une fois que l'on a remarqué que

$$
\max _{k \leq(g+1) C_{0}^{3} \mathfrak{a}} h\left(k \mathbf{p}_{i}\right) \leq \max _{\left.k \leq\left(\left[(g+1) C_{0}^{3}\right]+1\right)[\mathfrak{a}]\right)} h\left(k \mathbf{p}_{i}\right) \leq q \overline{39}\left(2(g+1) C_{0}^{3}\right)^{\rho_{i}}\left(1+\max _{k \leq \mathfrak{a}} h\left(k \mathbf{p}_{i}\right)\right)
$$

pour tout $i \in\{1, \ldots, n\}$. Cette dernière inégalité est une conséquence du lemme suivant. 
LEMME 8.28. Il existe une constante $\overline{3_{39}} \geq 1$ telle que pour tout couple d'entiers naturels non nuls $a_{1}, a_{2}$ et pour tout $i \in\{1, \ldots, n\}$, on ait

$$
\max _{k \leq a_{1} a_{2}} h\left(k \mathbf{p}_{i}\right) \leq q 39 a_{1}^{\rho_{i}}\left(1+\max _{k \leq a_{2}} h\left(k \mathbf{p}_{i}\right)\right) .
$$

Démonstration. On a

$$
\max _{k \leq a_{1} a_{2}} h\left(k \mathbf{p}_{i}\right)=\max \left\{\max _{k \leq a_{2}} h\left(k \mathbf{p}_{i}\right), \max _{a_{2}<k \leq a_{1} a_{2}} h\left(k \mathbf{p}_{i}\right)\right\} .
$$

Soit $k \in\left\{a_{2}+1, \ldots, a_{1} a_{2}\right\}$. Soient $q, r \in \mathbb{N}$ le quotient et le reste de la division euclidienne de $k$ par $a_{2}$, donc $k=q a_{2}+r, r<a_{2}$. Comme $k \leq a_{1} a_{2}, q$ est inférieur à $a_{1}$. D'après [41, propriété 4.5], il existe une constante $c \geq 1$ telle que

$$
h\left(k \mathbf{p}_{i}\right) \leq c \max \left\{1, h\left(q a_{2} \mathbf{p}_{i}\right), h\left(r \mathbf{p}_{i}\right)\right\} .
$$

D'après [19, page 144], il existe une constante $c^{\prime} \geq 1$ telle que

$$
h\left(q a_{2} \mathbf{p}_{i}\right) \leq c^{\prime} q^{\rho_{i}}\left(1+h\left(a_{2} \mathbf{p}_{i}\right)\right) \leq c^{\prime} a_{1}^{\rho_{i}}\left(1+\max _{0 \leq \ell \leq a_{2}} h\left(\ell \mathbf{p}_{i}\right)\right) .
$$

De plus, $h\left(r \mathbf{p}_{i}\right) \leq \max _{0 \leq \ell \leq a_{2}} h\left(\ell \mathbf{p}_{i}\right)$. On en déduit que

$$
h\left(k \mathbf{p}_{i}\right) \leq c c^{\prime} a_{1}^{\rho_{i}}\left(1+\max _{0 \leq \ell \leq a_{2}} h\left(\ell \mathbf{p}_{i}\right)\right)
$$

et donc

$$
\max _{k \leq a_{1} a_{2}} h\left(k \mathbf{p}_{i}\right) \leq c c^{\prime} a_{1}^{\rho_{i}}\left(1+\max _{\ell \leq a_{2}} h\left(\ell \mathbf{p}_{i}\right)\right)
$$

ce qui démontre le lemme.

8.11.2. Cas ultramétrique. Supposons que la place $v_{0}$ est finie et que le nombre réel $C_{0}$ est supérieur à $\max \left\{\left(\log p_{0}\right)^{2}, e^{2}, q_{21}, q_{22}, q_{32}^{2}, q_{35}\right\}$. Les propositions 8.22 et 8.24 entraînent

$$
-q_{36} \frac{U}{C_{0} D} \leq h(\mathcal{J}) \leq-\frac{U}{D}+\frac{2 q_{29} U}{\sqrt{C_{0}} D}+\aleph\left(\left(P_{\lambda_{0}}\right)_{\lambda_{0}}\right),
$$

d'où

$$
U \leq\left(q_{36}+2 q_{29}\right) \frac{U}{\sqrt{C_{0}}}+D \aleph\left(\left(P_{\lambda_{0}}\right)_{\lambda_{0}}\right)
$$

On pose $b=\left[S_{0} \log \Re /\left(C_{0} D\right)\right]$ et $P_{\lambda_{0}}=\delta_{b}\left(X ; \lambda_{0}\right)$ pour tout entier naturel $\lambda_{0} \leq D_{0}$. D'après la proposition 8.26 , on a

$$
\begin{aligned}
\aleph\left(\left(P_{\lambda_{0}}\right)_{\lambda_{0}}\right) & \leq q_{37}\left(D_{0} \log \left(e+\frac{S}{b}\right)+b T+\frac{D_{0}}{D} \log \left(1+\frac{\max \left\{S_{0}, \mathfrak{R}\right\}}{b}\right)\right) \\
& \leq q_{377}\left(D_{0} \log \left(e+\frac{2 S C_{0} D}{S_{0} \log \mathfrak{R}}\right)+\frac{1}{C_{0} D} T S_{0} \log \mathfrak{R}+\frac{D_{0}}{D} \log \left(1+\frac{\mathfrak{R} C_{0} D}{\log \mathfrak{R}}\right)\right) \\
& \leq q_{37}\left(2 \frac{A_{0} D_{0}}{D}+\frac{U}{C_{0} D}\right) \leq 3 q_{377} \frac{U}{C_{0} D} .
\end{aligned}
$$

On obtient finalement

$$
U \leq\left(q_{36}+2 q_{29}+3 q_{37}\right) \frac{U}{\sqrt{C_{0}}} .
$$

On a donc une contradiction dès que

$$
C_{0}>\max \left\{\left(\log p_{0}\right)^{2}, e^{2}, q_{21}, q_{22}, q_{32}^{2}, q_{35},\left(q_{36}+2 q_{29}+3 q_{37}\right)^{2}\right\} .
$$


On en déduit que l'hypothèse 2 ne peut pas être vérifiée et donc $d(u, V)>\exp \left(-2 \sqrt{C_{0}} U\right)$. Le théorème 4.4 découle alors d'une comparaison entre $U$ et la quantité $U_{3}$ du théorème, similaire à celle du cas archimédien.

\section{Démonstration dans le cas général}

Nous allons maintenant passer à la démonstration des théorèmes 4.1 et 4.2 . Le schéma de la preuve est identique, à la différence que nous n'ajouterons pas de facteur $G_{0}=\mathbb{G}_{\mathrm{a}}$ au cours de la démonstration. Les paramètres $A_{0}$ et $D_{0}$ n'interviennent plus ici, et la définition de $U$ est donc légèrement différente. La démonstration est ainsi simplifiée : par exemple, nous n'aurons pas à évaluer le « poids de la droite affine » comme au paragraphe 8.10. Certaines étapes nécessitent des modifications mineures (définition de $\widetilde{G}$, lemme de multiplicités, majoration du rang de $A_{0}$ ) et nous reproduirons les preuves adaptées en détails. En revanche, toutes les estimations analytiques de la démonstration ne seront que des cas particuliers de celles que nous avons déjà démontrées, pour lesquelles il nous suffira de poser $D_{0}=0$.

Dans la suite, nous supposons que l'hypothèse 1 est vérifiée : pour tout sous-groupe algébrique connexe $G^{\prime}$ de $G$ tel que $t_{G^{\prime}}+V \neq t_{G}$, on a $u \notin t_{G^{\prime}}\left(\mathbb{C}_{v_{0}}\right)$.

9.1. Choix d'un sous-groupe. Soient $\widetilde{D}_{1}, \ldots, \widetilde{D}_{n}, \widetilde{T}, C_{0}$ des nombres réels strictement positifs et $0<S_{0}<S$ des entiers. On suppose que $\widetilde{T}>1$ et on pose $T=[\widetilde{T}]$. Dans toute la suite, si $G^{\prime}$ est un sous-groupe algébrique de $G$, on note $r^{\prime}=\operatorname{codim}_{G} G^{\prime}$ et $\lambda^{\prime}=\operatorname{codim}_{V}\left(t_{G^{\prime}} \cap V\right)$. Posons $\Sigma_{\mathbf{p}}(S)=\left\{0_{G}, \mathbf{p}, \ldots, S \mathbf{p}\right\}$.

DÉfinition 9.1. Soit $G^{\prime}$ un sous-groupe algébrique connexe de $G$ tel que $t_{G^{\prime}}+V \neq t_{G}$. On définit le réel strictement positif

$$
A\left(G^{\prime}\right)=\left(\frac{\widetilde{T}^{\lambda^{\prime}} \operatorname{card}\left(\frac{\Sigma_{\mathbf{p}}(S)+G^{\prime}(\overline{\mathbb{Q}})}{G^{\prime}(\overline{\mathbb{Q}})}\right) \mathscr{H}\left(G^{\prime} ; \widetilde{D}_{1}, \ldots, \widetilde{D}_{n}\right)}{C_{0} \mathscr{H}\left(G ; \widetilde{D}_{1}, \ldots, \widetilde{D}_{n}\right)}\right)^{1 /\left(r^{\prime}-\lambda^{\prime}\right)}
$$

et on pose $B\left(G^{\prime}\right)=A\left(G^{\prime}\right)^{\left(r^{\prime}-\lambda^{\prime}\right) / r^{\prime}} \max \left\{1, A\left(G^{\prime}\right)\right\}^{\lambda^{\prime} / r^{\prime}}$.

Comme dans le cas semi-abélien, la quantité

$$
x:=\min \left\{B\left(G^{\prime}\right) \mid t_{G^{\prime}}+V \neq t_{G}\right\},
$$

où $G^{\prime}$ varie parmi les sous-groupes algébriques connexes $G^{\prime}$ de $G$ tel que $t_{G^{\prime}}+V \neq t_{G}$, est un nombre réel strictement positif. Fixons un sous-groupe $\widetilde{G}$ de $G$ tel que $B(\widetilde{G})=x$. Pour tout $i \in\{1, \ldots, n\}$, on pose également $D_{i}^{\#}=x \widetilde{D}_{i}, D_{i}=\left[D_{i}^{\#}\right]$ et $D_{i}^{\prime}=\max \left\{1, D_{i}\right\}$. La démonstration du lemme suivant est identique à celle du lemme 8.2 .

Lemme 9.2. Supposons que $x \leq 1$. Alors pour tout sous-groupe algébrique connexe $G^{\prime}$ de $G$ tel que $t_{G^{\prime}}+V \neq t_{G}$, l'inégalité suivante est vérifiée :

$$
\widetilde{T}^{\lambda^{\prime}} \operatorname{card}\left(\frac{\Sigma_{\mathbf{p}}(S)+G^{\prime}(\overline{\mathbb{Q}})}{G^{\prime}(\overline{\mathbb{Q}})}\right) \mathscr{H}\left(G^{\prime} ; D_{1}^{\#}, \ldots, D_{n}^{\#}\right) \geq C_{0} \mathscr{H}\left(G ; D_{1}^{\#}, \ldots, D_{n}^{\#}\right) .
$$

De plus, cette inégalité est une égalité pour $G^{\prime}=\widetilde{G}$. 
Comme dans le cas semi-abélien, le résultat suivant découle du théorème 5.1 et du lemme 9.2

Lemme 9.3. Supposons que $x \leq 1$. Si la place $v_{0}$ est finie, alors

$$
\operatorname{card}\left(\frac{\Sigma_{\mathbf{p}}(S)+\widetilde{G}(\overline{\mathbb{Q}})}{\widetilde{G}(\overline{\mathbb{Q}})}\right)=S+1 .
$$

Si $v_{0}$ est une place archimédienne, il existe une constante $40 \geq 1$ telle que si

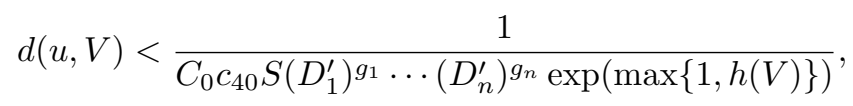

alors il n'existe pas d'entier $s \in\{1, \ldots, S\}$ tel que $s \mathbf{p} \in \widetilde{G}(\overline{\mathbb{Q}})$. En particulier

$$
\operatorname{card}\left(\frac{\Sigma_{\mathbf{p}}(S)+\widetilde{G}(\overline{\mathbb{Q}})}{\widetilde{G}(\overline{\mathbb{Q}})}\right)=S+1
$$

Démonstration. Si la place $v_{0}$ est finie, l'exponentielle exp réalise un difféomorphisme de $D\left(0, r_{p_{0}}\right)$ sur son image. S'il existe $s \in \mathbb{N} \backslash\{0\}$ tel que $s \mathbf{p} \in \widetilde{G}(\overline{\mathbb{Q}})$, on en déduit que $u \in t_{\widetilde{G}}\left(\mathbb{C}_{v_{0}}\right)$, ce qui est absurde d'après l'hypothèse 1 (par construction, on a $t_{\widetilde{G}}+V \neq t_{G}$ ). Supposons que la place $v_{0}$ est archimédienne. Nous allons appliquer le théorème 5.1 à $\mathcal{G}=G, \mathcal{H}=H$ et au point $u \in t_{G}$. L'hypothèse 1 correspond alors à celle du théorème 5.1 (1) Par définition de $\widetilde{G}$, on a $t_{\widetilde{G}}+V \neq t_{G}$. De plus, d'après le lemme 9.2, on a

$$
\widetilde{T}^{\widetilde{\lambda}} \operatorname{card}\left(\frac{\Sigma_{\mathbf{p}}(S)+\widetilde{G}(\overline{\mathbb{Q}})}{\widetilde{G}(\overline{\mathbb{Q}})}\right) \mathscr{H}\left(\widetilde{G} ; D_{1}^{\#}, \ldots, D_{n}^{\#}\right)=C_{0} \mathscr{H}\left(G ; D_{1}^{\#}, \ldots, D_{n}^{\#}\right) \text {. }
$$

D'après [28, propriété 4.4], pour tout $i \in\{0, \ldots, n\}$, l'application partielle

$$
x_{i} \mapsto \mathscr{H}\left(G ; x_{1}, \ldots, x_{n}\right) / \mathscr{H}\left(\widetilde{G} ; x_{1}, \ldots, x_{n}\right)
$$

est croissante. Comme $D_{i}^{\#} \leq 2 D_{i}^{\prime}$, on en déduit que

$$
\widetilde{T}^{\widetilde{\lambda}} \operatorname{card}\left(\frac{\Sigma_{\mathbf{p}}(S)+\widetilde{G}(\overline{\mathbb{Q}})}{\widetilde{G}(\overline{\mathbb{Q}})}\right) \mathscr{H}\left(\widetilde{G} ; D_{1}^{\prime}, \ldots, D_{n}^{\prime}\right) \leq C_{0} 2^{g} \mathscr{H}\left(G ; D_{1}^{\prime}, \ldots, D_{n}^{\prime}\right) \text {. }
$$

Comme $1 \leq D_{i}^{\prime}$ pour tout $i$, on a par ailleurs

$$
\operatorname{deg} \widetilde{G}=\mathscr{H}(\widetilde{G} ; 1, \ldots, 1) \leq \mathscr{H}\left(\widetilde{G} ; D_{1}^{\prime}, \ldots, D_{n}^{\prime}\right),
$$

et donc

$$
\operatorname{deg} \widetilde{G} \leq C_{0} 2^{g} \mathscr{H}\left(G ; D_{1}^{\prime}, \ldots, D_{n}^{\prime}\right)=C_{0} 2^{g} \operatorname{deg} G \cdot\left(D_{1}^{\prime}\right)^{g_{1}} \cdots\left(D_{n}^{\prime}\right)^{g_{n}} .
$$

On conclut alors avec le théorème 5.1 .1(1).

9.2. Fibré adélique hermitien des sections auxiliaires. Nous allons définir un fibré adélique $E$ de la même façon que dans le cas semi-abélien. Notons $\mathbb{P}=\mathbb{P}_{K}^{N_{1}} \times \cdots$ $\times \mathbb{P}_{K}^{N_{n}}$. Pour tout $i \in\{1, \ldots, n\}$, on fixe des coordonnées homogènes $\left(X_{i, j}\right)_{0 \leq j \leq N_{i}}$ de $\mathbb{P}_{K}^{N_{i}}$. Notons également $\mathbf{D}=\left(D_{1}, \ldots, D_{n}\right)$ et $K[\mathbb{P}]$ la $K$-algèbre multigraduée des polynômes multihomogènes en les variables $\left(X_{i, j}\right)_{1 \leq i \leq n, 0 \leq j \leq N_{i}}$. Soit $E$ l'espace des polynômes de multidegré $\mathbf{D}$ qui ne s'annulent pas identiquement sur $G$; en notant $I_{G}$ l'idéal annulateur 
de $G$ dans $\mathbb{P}$, on a ainsi $E=\left(K[\mathbb{P}] / I_{G}\right)_{\mathbf{D}}$. Considérons l'ensemble

$$
\Lambda=\left\{\boldsymbol{\lambda}=\left(\boldsymbol{\lambda}_{i}\right)_{1 \leq i \leq n} \in \prod_{i=1}^{n} \mathbb{N}^{N_{i}+1}\left|\boldsymbol{\lambda}_{i}=\left(\lambda_{i, j}\right)_{0 \leq j \leq N_{i}},\right| \boldsymbol{\lambda}_{i} \mid=D_{i}\right\} .
$$

L'ensemble des classes de polynômes de la forme $\prod_{i, j} X_{i, j}^{\lambda_{i, j}}, \boldsymbol{\lambda} \in \Lambda$, est une famille génératrice de $E$. Considérons une famille de tels polynômes dont les classes $s_{1}, \ldots, s_{\operatorname{dim}} E$ forment une base de $E$. On définit alors un fibré adélique hermitien $\left(E,\left(\|\cdot\|_{E, v}\right)_{v \in K}\right)$ en choisissant pour chaque place $v \in \Sigma_{K}$ la norme $\|\cdot\|_{E, v}$ rendant cette base orthonormée. On a ainsi $\widehat{\mu}_{\mathrm{n}}(E)=0$. Concrètement, un élément $s$ de $E$ s'écrit comme la classe d'équivalence d'un polynôme

$$
P=\sum_{\boldsymbol{\lambda} \in \Lambda} p_{\boldsymbol{\lambda}} \prod_{i=1}^{n} \prod_{j=0}^{N_{i}} X_{i, j}^{\lambda_{i, j}}
$$

On note alors $F_{s}=P \circ \Psi: \mathscr{U} \rightarrow \mathbb{C}_{v_{0}}$. Cette application est bien définie car elle ne dépend pas du choix d'un polynôme $P$ représentant $s$. Nous allons maintenant définir une norme particulière en la place $v_{0}$. Rappelons que $S_{0}>0$ est un entier et que $T=[\widetilde{T}]$. On considère l'ensemble

$$
\Upsilon=\left\{(m, \boldsymbol{\tau}) \in \mathbb{N} \times \mathbb{N}^{g-t}\left|m \leq S_{0},\right| \boldsymbol{\tau} \mid \leq 2 g T\right\} .
$$

Soit $\mathbf{w}=\left(w_{1}, \ldots, w_{g-t}\right)$ une base de $V \otimes_{K} \mathbb{C}_{v_{0}}$ formée de vecteurs de normes 1 . Considérons la matrice $\mathcal{A}_{0}$ de taille card $\boldsymbol{\Upsilon} \times \operatorname{dim} E$ définie par : pour tout $(m, \boldsymbol{\tau}) \in \boldsymbol{\Upsilon}$ et tout $i \in\{1, \ldots, \operatorname{dim} E\}$,

$$
\mathcal{A}_{0}[(m, \boldsymbol{\tau}), i]=\frac{1}{\boldsymbol{\tau} !} D_{\mathbf{w}}^{\boldsymbol{\tau}} F_{s_{i}}(m u) .
$$

Étant donné un nombre réel $\alpha>0$ et une place $v$ de $K$, on définit la norme $\|\cdot\|_{\alpha, v}$ sur $E \otimes_{K} \mathbb{C}_{v}$ en posant $\|\cdot\|_{\alpha, v}=\|\cdot\|_{E, v}$ si $v \neq v_{0}$ et

$$
\|s\|_{\alpha, v_{0}}= \begin{cases}\max \left\{\|s\|_{E, v_{0}}, \alpha\left|\mathcal{A}_{0} s\right|_{v_{0}}\right\} & \text { si } v_{0} \text { est finie } \\ \left(\|s\|_{E, v_{0}}^{2}+\left(\alpha\left|\mathcal{A}_{0} s\right|_{v_{0}}\right)^{2}\right)^{1 / 2} & \text { sinon. }\end{cases}
$$

On note $h_{\alpha}$ la hauteur (logarithmique et absolue) sur $E \otimes_{K} \overline{\mathbb{Q}}$ associée à cette famille de normes. Remarquons que pour toute place $v \in \Sigma_{K}$, on a l'inégalité $\|\cdot\|_{E, v} \leq\|\cdot\|_{\alpha, v}$.

9.3. Lemme de multiplicités. Nous allons maintenant énoncer une conséquence du lemme de multiplicités de Philippon [33. La preuve diffère légèrement de celle du cas semi-abélien, et les conditions (a) et (c) de l'énoncé ci-dessous ne sont pas les mêmes que celles du lemme 8.4. Cette différence nous conduira à faire un nouveau choix de paramètres au paragraphe 9.4 .

LEMme 9.4. Supposons que $x \leq 1$. Il existe une constante $41 \geq 1$ vérifiant la propriété suivante. Si les conditions

(a) $\left(q_{22} S\left(D_{1}^{\prime}\right)^{g_{1}} \cdots\left(D_{n}^{\prime}\right)^{g_{n}}\right)^{-1}>d(u, V)$;

(b) $\min \{T, S\}>C_{0}>$ q41,

(c) $T>41$ max $\left\{D_{1}^{\prime}, \ldots, D_{n}^{\prime}\right\}$ 
sont vérifiées, alors il n'existe pas d'élément $s \in E \otimes_{K} \overline{\mathbb{Q}} \backslash\{0\}$ telle que

$$
\mathcal{D}_{\mathbf{w}}^{\boldsymbol{\tau}} F_{s}(m, m u)=0 \quad \forall(m, \boldsymbol{\tau}) \in \mathbb{N} \times \mathbb{N}^{g-t}, m \leq g S,|\boldsymbol{\tau}| \leq g T .
$$

Démonstration. Nous raisonnons par l'absurde en supposant qu'il existe une solution $s \in E \otimes_{K} \overline{\mathbb{Q}} \backslash\{0\}$ au système d'égalités $(9.3)$. D'après le lemme de multiplicités de Philippon [33], il existe un sous-groupe algébrique connexe $G^{\prime} \subsetneq G$ tel que

$$
T^{\lambda^{\prime}} \operatorname{card}\left(\frac{\Sigma_{\mathbf{p}}(S)+G^{\prime}(\overline{\mathbb{Q}})}{G^{\prime}(\overline{\mathbb{Q}})}\right) \mathscr{H}\left(G^{\prime} ; D_{1}^{\prime}, \ldots, D_{n}^{\prime}\right) \leq 2^{g} \mathscr{H}\left(G ; D_{1}^{\prime}, \ldots, D_{n}^{\prime}\right) \text {. }
$$

Étape 1: Montrons que $t_{G^{\prime}}+V \neq t_{G}$. Par l'absurde, si $t_{G^{\prime}}+V=t_{G}$ alors $\lambda^{\prime}=r^{\prime}=$ $\operatorname{codim}_{G} G^{\prime}$ et on en déduit que

$$
T^{r^{\prime}} \leq 2^{g} \operatorname{deg} G \max \left\{D_{1}^{\prime}, \ldots, D_{n}^{\prime}\right\}^{r^{\prime}},
$$

ce qui contredit la condition (c) pourvu que l'on choisisse la constante 441 assez grande.

Étape 2: Montrons que $\lambda^{\prime} \geq 1$ ou bien que pour tout $m \in\{1, \ldots, S\}, m \mathbf{p} \notin G^{\prime}(K)$. D'après la première étape, $t_{G^{\prime}}+V \neq t_{G}$, donc d'après l'hypothèse 1 on a $u \notin t_{G^{\prime}}\left(\mathbb{C}_{v_{0}}\right)$. Si $\lambda^{\prime}=\operatorname{codim}_{V}\left(t_{G}^{\prime} \cap V\right)=0$, alors $V \subseteq t_{G}^{\prime}$. D'après l'inégalité (9.4), on a

$$
\operatorname{deg} G^{\prime} \leq 2^{g} \operatorname{deg} G \cdot\left(D_{1}^{\prime}\right)^{g_{1}} \cdots\left(D_{n}^{\prime}\right)^{g_{n}} .
$$

D'après la condition (a), on en déduit que

$$
d(u, V) \leq \frac{2^{g} \operatorname{deg} G}{q 41 S \operatorname{deg} G^{\prime}} .
$$

En appliquant le second point du théorème 5.1 au vecteur $u$ avec $\mathcal{G}=G, \mathcal{G}^{\prime}=G^{\prime}, \mathcal{H}=H$, on en déduit que pour tout $0<m \leq S, m \mathbf{p} \notin G^{\prime}(K)$ (pourvu que la constante q41 soit choisie suffisamment grande).

Étape 3 : Nous allons conclure en montrant que l'inégalité (9.4) contredit le lemme 9.2 . Premièrement, remarquons qu'il existe $i \in\{1, \ldots, n\}$ tel que $D_{i} \neq 0$. En effet, sinon tous les $D_{i}^{\prime}$ sont égaux à 1 et l'inégalité (9.4) entraîne

$$
T^{\lambda^{\prime}} \operatorname{card}\left(\frac{\Sigma_{\mathbf{p}}(S)+G^{\prime}(\overline{\mathbb{Q}})}{G^{\prime}(\overline{\mathbb{Q}})}\right) \leq 2^{g} \operatorname{deg} G .
$$

D'après l'étape 2 , on obtient $\min \{T, S+1\} \leq 2^{g} \operatorname{deg}(G)$, ce qui contredit la condition (b). Il existe donc au moins un $i$ tel que $D_{i} \neq 0$.

On considère alors les entiers $1 \leq k_{1}<\cdots<k_{h} \leq n$ pour lesquels $D_{k_{i}} \neq 0,1 \leq i \leq h$, et on note $\pi$ la projection

$$
\pi: G \rightarrow \prod_{i=1}^{h} G_{k_{i}}
$$

On montre que

$$
\mathscr{H}\left(G ; D_{1}^{\prime}, \ldots, D_{n}^{\prime}\right) \leq \operatorname{deg} G \cdot \mathscr{H}\left(\pi(G) ; D_{k_{1}}, \ldots, D_{k_{h}}\right)
$$

comme dans la preuve du lemme 8.4. On a également

$$
\mathscr{H}\left(\pi\left(G^{\prime}\right) ; D_{k_{1}}, \ldots, D_{k_{h}}\right) \leq \mathscr{H}\left(G^{\prime} ; D_{1}^{\prime}, \ldots, D_{n}^{\prime}\right) .
$$


En utilisant l'inégalité $(9.4)$, on obtient

$$
T^{\lambda^{\prime}} \operatorname{card}\left(\frac{\Sigma_{\mathbf{p}}(S)+G^{\prime}(\overline{\mathbb{Q}})}{G^{\prime}(\overline{\mathbb{Q}})}\right) \leq 2^{g} \operatorname{deg} G \cdot \frac{\mathscr{H}\left(\pi(G) ; D_{k_{1}}, \ldots, D_{k_{h}}\right)}{\mathscr{H}\left(\pi\left(G^{\prime}\right) ; D_{k_{1}}, \ldots, D_{k_{h}}\right)} .
$$

Puisque $D_{i}=\left[D_{i}^{\#}\right] \leq D_{i}^{\#}$ pour tout $i \in\{1, \ldots, n\}$, on obtient l'inégalité

$$
T^{\lambda^{\prime}} \operatorname{card}\left(\frac{\Sigma_{\mathbf{p}}(S)+G^{\prime}(\overline{\mathbb{Q}})}{G^{\prime}(\overline{\mathbb{Q}})}\right) \leq 2^{g} \operatorname{deg} G \cdot \frac{\mathscr{H}\left(\pi(G) ; D_{k_{1}}^{\#}, \ldots, D_{k_{h}}^{\#}\right)}{\mathscr{H}\left(\pi\left(G^{\prime}\right) ; D_{k_{1}}^{\#}, \ldots, D_{k_{h}}^{\#}\right)}
$$

par croissance des applications partielles

$$
x_{i} \mapsto \mathscr{H}\left(\pi(G) ; x_{k_{1}}, \ldots, x_{k_{h}}\right) / \mathscr{H}\left(\pi\left(G^{\prime}\right) ; x_{k_{1}}, \ldots, x_{k_{h}}\right)
$$

(voir [28, propriété 4.4]). On considère le groupe $G^{\prime \prime}$ (vu comme un sous-groupe de $G$ après permutation éventuelle des facteurs) défini par

$$
G^{\prime \prime}=\pi\left(G^{\prime}\right) \times \prod_{j \notin\left\{k_{1}, \ldots, k_{h}\right\}} G_{j} .
$$

Le sous-groupe $G^{\prime \prime}$ est distinct de $G$, car sinon $\pi\left(G^{\prime}\right)=\pi(G)$ et l'inégalité (9.5) ainsi que l'étape 2 entraînent $\min \{T, S+1\} \leq 2^{g} \operatorname{deg} G$, ce qui contredit la condition (b) pourvu que $q 41 \geq 2^{g} \operatorname{deg} G$. On a par ailleurs

$$
\frac{\mathscr{H}\left(\pi(G) ; D_{k_{1}}^{\#}, \ldots, D_{k_{h}}^{\#}\right)}{\mathscr{H}\left(\pi\left(G^{\prime}\right) ; D_{k_{1}}^{\#}, \ldots, D_{k_{h}}^{\#}\right)}=\frac{\left(\operatorname{dim} G^{\prime \prime}\right) !(\operatorname{dim} \pi(G)) !}{g !\left(\operatorname{dim} \pi\left(G^{\prime}\right)\right) !} \cdot \frac{\mathscr{H}\left(G ; D_{1}^{\#}, \ldots, D_{n}^{\#}\right)}{\mathscr{H}\left(G^{\prime \prime} ; D_{1}^{\#}, \ldots, D_{n}^{\#}\right)}
$$

et

- $\operatorname{dim} \pi(G)-\operatorname{dim} \pi\left(G^{\prime}\right)=\operatorname{codim}_{G} G^{\prime \prime}=r^{\prime \prime}$,

- $\lambda^{\prime \prime}=\operatorname{codim}_{V}\left(V \cap t_{G^{\prime \prime}}\right) \leq \operatorname{codim}_{V}\left(V \cap t_{G^{\prime}}\right)=\lambda^{\prime}\left(\operatorname{car} G^{\prime} \subseteq G^{\prime \prime}\right)$,

- $\operatorname{card}\left(\frac{\Sigma_{\mathbf{p}}(S)+G^{\prime \prime}(\overline{\mathbb{Q}})}{G^{\prime \prime}(\overline{\mathbb{Q}})}\right) \leq \operatorname{card}\left(\frac{\Sigma_{\mathbf{p}}(S)+G^{\prime}(\overline{\mathbb{Q}})}{G^{\prime}(\overline{\mathbb{Q}})}\right)$.

D'après l'inégalité 9.5 , on en déduit qu'il existe une constante $c$ ne dépendant que de $g$ telle que

$T^{\lambda^{\prime \prime}} \operatorname{card}\left(\frac{\Sigma_{\mathbf{p}}(S)+G^{\prime \prime}(\overline{\mathbb{Q}})}{G^{\prime \prime}(\overline{\mathbb{Q}})}\right) \mathscr{H}\left(G^{\prime \prime} ; D_{1}^{\#}, \ldots, D_{n}^{\#}\right) \leq c \operatorname{deg} G \cdot \mathscr{H}\left(G ; D_{1}^{\#}, \ldots, D_{n}^{\#}\right)$.

Si $C_{0}>(c \operatorname{deg} G)^{2}$, on montre que $t_{G^{\prime \prime}}+V \neq t_{G}$ en utilisant les mêmes arguments qu'à l'étape 1. On en déduit que si la constante 41 est suffisamment grande, l'inégalité (9.6) contredit le lemme 9.2 , ce qui achève la démonstration.

9.4. Choix des paramètres. Nous allons maintenant fixer tous les paramètres introduits précédemment. Rappelons que l'on a noté $D=[K: \mathbb{Q}] /\left[K_{v_{0}}: \mathbb{Q}_{v_{0}}\right]$. Dans toute la suite, $C_{0}$ désigne un nombre réel supérieur à $\max \{440,441\} \geq 1$. Si $v_{0} \mid p_{0}$ est ultramétrique, on suppose également que $C_{0}>e^{2}$.

9.4.1. Cas archimédien. Supposons que la place $v_{0}$ est archimédienne. Soit $\mathfrak{e} \geq e$ un nombre réel et soit $\mathfrak{a} \geq 1$ un nombre réel tel que

$$
\mathfrak{a} \geq \frac{\max \left\{D, D h(V), \log \|u\|_{v_{0}}\right\}}{\log \mathfrak{e}} .
$$


Posons alors $S_{0}:=\left[C_{0} \mathfrak{a}\right]$ et $S:=\left[C_{0}^{3} \mathfrak{a}\right]$. On définit également

$$
\begin{aligned}
A_{i} & :=D \max _{k \leq(g+1) S} h\left(k \mathbf{p}_{i}\right)+\left(1+C_{0}^{3} \mathfrak{a} \mathfrak{e}\left\|u_{i}\right\|_{v_{0}}\right)^{\rho_{i}}, \quad i \in\{1, \ldots, n\}, \\
U & :=C_{0} S_{0} \log \mathfrak{e}(S+1)^{1 / t} \prod_{i=1}^{n}\left(C_{0}^{2}+\frac{C_{0} A_{i}}{S_{0} \log \mathfrak{e}}\right)^{g_{i} / t} .
\end{aligned}
$$

On pose alors

$$
\widetilde{T}:=\frac{U}{S_{0} \log \mathfrak{e}}, \quad \widetilde{D}_{i}:=\frac{U}{C_{0} A_{i}+C_{0}^{2} S_{0} \log \mathfrak{e}}, \quad i \in\{1, \ldots, n\} .
$$

Rappelons que l'on a noté $T=[\widetilde{T}], D_{i}=\left[x \widetilde{D}_{i}\right]$ et $D_{i}^{\prime}=\max \left\{1, D_{i}\right\}$ pour tout $i$. Nous aurons besoin du lemme suivant.

LEMME 9.5. Les inégalités suivantes sont vérifiées :

(1) $x \leq 1$;

(2) $\widetilde{T}>C_{0}^{2}, T>C_{0}$;

(3) $\left(S_{0}+1\right) /(S+1) \leq 2 / C_{0}^{2}, S / S_{0} \leq 2 C_{0}^{2}$;

(4) $T>C_{0} \max \left\{D_{1}^{\prime}, \ldots, D_{n}^{\prime}\right\}$;

(5) $D_{i} \leq D_{i}^{\prime} \leq \widetilde{D}_{i} \leq \widetilde{D}_{i} A_{i} \leq U / C_{0} \forall i \in\{1, \ldots, n\}$;

(6) $\widetilde{D}_{i} \max _{k \leq(g+1) S} h\left(k \mathbf{p}_{i}\right) \leq U /\left(D C_{0}\right) \forall i \in\{1, \ldots, n\}$;

(7) $\widetilde{D}_{i}\left(1+S \mathfrak{e}\left\|u_{i}\right\|_{v_{0}}\right)^{\rho_{i}} \leq U / C_{0} \forall i \in\{1, \ldots, n\}$.

Démonstration. Montrons le premier point. Par définition de $x$, on a l'implication

$$
\frac{1}{\operatorname{deg} G} \frac{\widetilde{T}^{g-t}(S+1)}{C_{0} \widetilde{D}_{1}^{g_{1}} \cdots \widetilde{D}_{n}^{g_{n}}}=A(\{0\})^{t} \leq 1 \Longrightarrow x \leq 1 .
$$

La définition de $U$ correspond précisément à $\operatorname{deg} G \cdot A(\{0\})^{t}=C_{0}^{-(t+1)} \leq 1$, ce qui démontre le point (1) Les points suivants sont des conséquences immédiates des définitions et de l'inégalité $x \leq 1$.

9.4.2. Cas ultramétrique. Supposons que la place $v_{0}$ est ultramétrique. Soit $\mathfrak{R} \in$ ] $1, r_{p_{0}} /\|u\|_{v_{0}}[$ et soit $\mathfrak{a} \geq 1$ tel que

$$
\mathfrak{a} \log \mathfrak{R} \geq D \max \{1, h(V)\}+\log _{+}\left((\log \mathfrak{R})^{-1}\right) .
$$

Posons $S_{0}:=\left[C_{0} \mathfrak{a}\right]$ et $S:=\left[C_{0}^{3} \mathfrak{a}\right]$. On définit également les quantités

$$
\begin{aligned}
A_{i} & :=D \max _{k \leq(g+1) S} h\left(k \mathbf{p}_{i}\right), \quad i \in\{1, \ldots, n\} \\
U & :=C_{0} S_{0}(\log \Re)(S+1)^{1 / t} \prod_{i=1}^{n}\left(C_{0}^{2}+\frac{C_{0} A_{i}}{S_{0} \log \Re}\right)^{g_{i} / t} .
\end{aligned}
$$

On pose alors

$$
\widetilde{T}:=\frac{U}{S_{0} \log \Re}, \quad \widetilde{D}_{i}:=\frac{U}{C_{0} A_{i}+C_{0}^{2} S_{0} \log \Re}, \quad i \in\{1, \ldots, n\} .
$$

Rappelons que l'on a noté $T=[\widetilde{T}], D_{i}=\left[x \widetilde{D}_{i}\right]$ et $D_{i}^{\prime}=\max \left\{1, D_{i}\right\}$ pour tout $i \in$ $\{1, \ldots, n\}$. Comme dans le cas archimédien, énumérons quelques propriétés utiles satisfaites par ces paramètres. 
LEMME 9.6. Les inégalités suivantes sont vérifiées :

(1) $x \leq 1$;

(2) $\widetilde{T}>C_{0}^{2}, T>C_{0}$;

(3) $\left(S_{0}+1\right) /(S+1) \leq 2 / C_{0}^{2}, S / S_{0} \leq 2 C_{0}^{2}$;

(4) $T>C_{0} \max \left\{D_{1}^{\prime}, \ldots, D_{n}^{\prime}\right\}$;

(5) $D_{i} \leq D_{i}^{\prime} \leq \widetilde{D}_{i} \leq \widetilde{D}_{i} A_{i} \leq U / C_{0} \forall i \in\{1, \ldots, n\}$;

(6) $\widetilde{D}_{i} \max _{k \leq(g+1) S} h\left(k \mathbf{p}_{i}\right) \leq U /\left(D C_{0}\right) \forall i \in\{1, \ldots, n\}$;

(7) $S_{0} \log \mathfrak{R} \geq \log S_{0}$.

9.4.3. Hypothèse centrale. Nous allons raisonner par l'absurde afin de démontrer une minoration de la distance $d(u, V)$. Dans toute la suite, on fait l'hypothèse suivante, qui implique en particulier que les conclusions des lemmes 9.3 et 9.4 sont satisfaites.

Hypothèse 3. La distance $d(u, V)$ est inférieure à $\exp \left(-2 \sqrt{C_{0}} U\right)$.

Dans toute la suite, on pose $\alpha:=\exp \left(U\left(g+\sqrt{C_{0}}\right)\right)$.

Remarque importante : Dans la suite du texte, nous allons établir des estimations analytiques dont les démonstrations sont les mêmes que celles du cas semi-abélien, appliquées avec $D_{0}=0$. Nous renverrons systématiquement aux démonstrations correspondantes du paragraphe 8. Bien que notre définition de $U$ soit légèrement différente, les seules propriétés des paramètres dont nous avons besoin dans les preuves sont celles des lemmes 9.5 et 9.6 , qui sont exactement les mêmes que celles des lemmes 8.5 et 8.6.

\section{5. Étude de la matrice $\mathcal{A}_{0}$}

9.5.1. Majoration du rang. Le rang $\operatorname{rg} \mathcal{A}_{0}$ de la matrice $\mathcal{A}_{0}$ est égal au rang du système

$$
\forall(m, \boldsymbol{\tau}) \in \boldsymbol{\Upsilon}, \quad D_{\mathbf{w}}^{\boldsymbol{\tau}} F_{s}(m u)=0
$$

en les inconnues $p_{i}$ définies par $s=\sum_{i=1}^{\operatorname{dim} E} p_{i} s_{i}$. On note $\widetilde{\lambda}=\operatorname{codim}_{V}\left(V \cap t_{\widetilde{G}}\right)$. Les arguments de la démonstration du lemme 6.7 de [35] montrent que ce rang est inférieur à celui du système

$$
\forall(m, \boldsymbol{\tau}) \in \mathbb{N} \times \mathbb{N}^{\tilde{\lambda}}, 0 \leq m \leq S_{0},|\boldsymbol{\tau}| \leq 2 g T, \quad D_{\mathbf{w}^{\prime}}^{\boldsymbol{\tau}} P(m \mathbf{p}+\widetilde{G})=0,
$$

où $\mathbf{w}^{\prime}$ désigne une base d'un supplémentaire de $V \cap t_{\widetilde{G}}$ dans $V$ si $\widetilde{\lambda} \geq 1$ (voir aussi la démonstration du lemme 6.1 de [14]), et où les inconnues du système sont les coordonnées des polynômes $P \in K[\mathbb{P}]_{\mathbf{D}}$ (dans une base quelconque de $K[\mathbb{P}]_{\mathbf{D}}$ ). On en déduit que

$$
\operatorname{rg} \mathcal{A}_{0} \leq \operatorname{card}\left\{\boldsymbol{\tau} \in \mathbb{N}^{\tilde{\lambda}}|| \boldsymbol{\tau} \mid \leq 2 g T\right\} \operatorname{card}\left(\frac{\Sigma_{\mathbf{q}}\left(S_{0}\right)+\widetilde{G}(\overline{\mathbb{Q}})}{\widetilde{G}(\overline{\mathbb{Q}})}\right) \operatorname{dim}\left(\overline{\mathbb{Q}}[\mathbb{P}] / I_{\widetilde{G}}\right)_{2 \mathbf{D}},
$$

où $I_{\widetilde{G}}$ désigne l'idéal des polynômes identiquement nuls sur $\widetilde{G}$. Un raisonnement analogue à celui du paragraphe 8.5.1 conduit à l'existence d'une constante $42 \geq 1$ telle que

$$
\operatorname{rg} \mathcal{A}_{0} \leq C_{0} \frac{\operatorname{card}\left(\frac{\Sigma_{\mathbf{p}}\left(S_{0}\right)+\widetilde{G}(\overline{\mathbb{Q}})}{\widetilde{G}(\overline{\mathbb{Q}})}\right)}{\operatorname{card}\left(\frac{\Sigma_{\mathbf{p}}(S)+\widetilde{G}(\overline{\mathbb{Q}})}{\widetilde{G}(\overline{\mathbb{Q}})}\right)} \operatorname{dim} E .
$$

En appliquant le lemme 9.3 on obtient la la proposition suivante. 
Proposition 9.7. Il existe une constante q42 telle que

$$
\frac{\operatorname{rg} \mathcal{A}_{0}}{\operatorname{dim} E} \leq q_{42} C_{0} \frac{S_{0}+1}{S+1} \leq \frac{2 q_{42}}{C_{0}} .
$$

9.5.2. Majoration de la norme d'opérateur de $\mathcal{A}_{0}$. Nous allons maintenant majorer la quantité

$$
\left\|\mathcal{A}_{0}\right\|_{v_{0}}=\sup \left\{\frac{\left|\mathcal{A}_{0} s\right|_{v_{0}}}{\|s\|_{E, v_{0}}} \mid s \in E \otimes_{K} \mathbb{C}_{v_{0}}\right\} .
$$

La majoration repose sur les mêmes arguments que dans le cas semi-abélien. Soit $\mathbf{v}=$ $\left(v_{1}, \ldots, v_{g-t}\right)$ une base de $V \otimes_{K} \mathbb{C}_{v_{0}}$ telle que $\left\|v_{i}\right\|_{v_{0}} \leq 1$ pour tout $i \in\{1, \ldots, g-t\}$. Le résultat suivant correspond au lemme 8.9 dans le cas où $D_{0}=0$.

Lemme 9.8. Soit $\boldsymbol{\tau}=\left(\tau_{1}, \ldots, \tau_{g-t}\right) \in \mathbb{N}^{g-t}$ et soit $s \in E \otimes_{K} \mathbb{C}_{v_{0}}$. Si v $v_{0}$ est archimédienne, alors pour tout $z \in \mathbb{C}_{v_{0}}^{g_{1}} \times \cdots \times \mathbb{C}_{v_{0}}^{g_{n}} \simeq t_{G}\left(\mathbb{C}_{v_{0}}\right)$,

$$
\left|\frac{1}{\boldsymbol{\tau} !} \mathcal{D}_{\mathbf{v}}^{\boldsymbol{\tau}} F_{s}(z)\right|_{v_{0}} \leq\left(g^{2}-1\right)^{|\boldsymbol{\tau}|} \prod_{i=1}^{n} \exp \left(q_{43} D_{i}\left(1+\left\|z_{i}\right\|_{v_{0}}\right)^{\rho_{i}}\right)\|s\|_{E, v_{0}}
$$

où $z_{i}$ désigne la projection de z sur $\mathbb{C}_{v_{0}}^{g_{i}}$ et $\Psi_{43} \geq 1$ est une constante. Si $v_{0}$ est une place finie, alors pour tout $z \in D\left(0, r_{p_{0}}\right)$,

$$
\left|\frac{1}{\boldsymbol{\tau} !} \mathcal{D}_{\mathbf{v}}^{\boldsymbol{\tau}} F_{s}(z)\right|_{v_{0}} \leq\|s\|_{E, v_{0}} .
$$

La proposition suivante est une conséquence immédiate de la définition de la norme d'opérateur et des lemmes 9.5 et 9.8 .

Proposition 9.9. Si $v_{0}$ est une place archimédienne, il existe une constante $44 \geq 1$ telle que la norme d'opérateur de $\mathcal{A}_{0}$ vérifie

$$
\left\|\mathcal{A}_{0}\right\|_{v_{0}} \leq \exp \left(q 44_{44} U / C_{0}\right) .
$$

Si la place $v_{0}$ est ultramétrique, alors $\left\|\mathcal{A}_{0}\right\|_{v_{0}} \leq 1$.

9.6. Construction d'une section auxiliaire. Les majorations du paragraphe 9.5 nous permettent d'appliquer le lemme de Siegel du paragraphe 2 pour obtenir la proposition suivante.

Proposition 9.10. Il existe une constante $q_{45}$ et une section $s \in E \otimes_{K} \overline{\mathbb{Q}} \backslash\{0\}$ telle que

$$
h_{\alpha}(s) \leq \frac{4 \overline{45}}{D \sqrt{C_{0}}} U \text {. }
$$

Démonstration. La preuve est en tout point similaire à celle de la proposition 8.11 : il suffit d'appliquer le lemme de Siegel 2.14 et les propositions 9.7 et 9.9 .

9.7. Construction d'un jet et premières estimations. Considérons un élément non nul $s \in E \otimes_{K} \overline{\mathbb{Q}}$ dont la hauteur $h_{\alpha}(s)$ vérifie la majoration de la proposition 9.10 . Soit $(m, \ell) \in \mathbb{N} \times \mathbb{N}$ le couple minimal (pour l'ordre lexicographique) pour lequel il existe un élément $\boldsymbol{\tau}$ de $\mathbb{N}^{g-t}$ tel que $|\tau|=\ell$ et $\mathcal{D}_{\mathbf{w}}^{\boldsymbol{\tau}} F_{s}(m u) \neq 0$. D'après le lemme de multiplicités 8.4 . on a $m \leq g S$ et $\ell \leq g T$. Soit $K^{\prime}$ une extension finie de $K$ telle que $s \in E \otimes_{K} K^{\prime}$. Pour 
tout $i \in\{1, \ldots, n\}$, on choisit un entier $\varepsilon_{i} \in\left\{0, \ldots, N_{i}\right\}$ vérifiant

$$
\left|\varphi_{i, \varepsilon_{i}}\left(m u_{i}\right)\right|_{v_{0}}=\max _{0 \leq j \leq N_{i}}\left|\varphi_{i, j}\left(m u_{i}\right)\right|_{v_{0}} .
$$

En particulier $\varphi_{i, \varepsilon_{i}}\left(m u_{i}\right) \neq 0$. Soit $\mathbf{b}=\left(b_{1}, \ldots, b_{g-t}\right)$ une $K$-base de $V$. On note $\mathbf{b}^{\vee}$ la base duale de $\mathbf{b}$. On considère alors l'élément $\mathcal{J}$ de $S^{\ell}\left(V^{\vee}\right) \otimes_{K} \mathbb{C}_{v_{0}}$ défini par

$$
\mathcal{J}=\sum_{\substack{\boldsymbol{\tau} \in \mathbb{N}^{g-t} \\|\boldsymbol{\tau}|=\ell}} \prod_{i=1}^{n} \varphi_{i, \varepsilon_{i}}\left(m u_{i}\right)^{-D_{i}} \frac{1}{\boldsymbol{\tau} !} \mathcal{D}_{\mathbf{b}}^{\boldsymbol{\tau}} F_{s}(m u)\left(\mathbf{b}^{\vee}\right)^{\boldsymbol{\tau}}
$$

Les résultats du paragraphe 8.7 (appliqués avec $D_{0}=0$ ) montrent que $\mathcal{J}$ ne dépend pas du choix de la base b et que $\mathcal{J}$ appartient à $S^{\ell}\left(V^{\vee}\right) \otimes_{K} K^{\prime}$. On munit l'espace vectoriel $S^{\ell}\left(V^{\vee}\right) \otimes_{K} K^{\prime}$ de la structure de fibré adélique hermitien induite par celle de $V$. Nous disposons également de la majoration suivante.

Proposition 9.11. On a l'inégalité

$$
\sum_{\substack{v \in \Sigma_{K^{\prime}} \\ v \nmid v_{0}}} \frac{\left[K_{v}^{\prime}: \mathbb{Q}_{v}\right]}{\left[K^{\prime}: \mathbb{Q}\right]} \log \|\mathcal{J}\|_{v} \leq \sum_{\substack{v \in \Sigma_{K^{\prime}} \\ v \nmid v_{0}}} \frac{\left[K_{v}^{\prime}: \mathbb{Q}_{v}\right]}{\left[K^{\prime}: \mathbb{Q}\right]} \log \|s\|_{\alpha, v}+\frac{U}{\bar{D} C_{0}},
$$

où $q_{46}=q_{32} \geq 1$ est la constante de la proposition 8.17 .

Démonstration. La démonstration est identique à celle de la proposition 8.17 (il suffit de poser $\left.D_{0}=0\right)$.

Afin d'obtenir une majoration de la hauteur de $\mathcal{J}$, nous devons maintenant majorer $\|\mathcal{J}\|_{v}$ pour toute place $v$ de $K^{\prime}$ au-dessus de $v_{0}$. Afin d'aboutir à une contradiction, cette étape nécessite une étude minutieuse et fait l'objet de la partie suivante.

9.8. Majorations en les places $v \mid v_{0}$. Soit $v \in \Sigma_{K^{\prime}}$ une place divisant $v_{0}$. Comme dans le cas semi-abélien, la majoration de $\|\mathcal{J}\|_{v}$ repose sur une extrapolation sur les points. On peut supposer sans perte de généralité que la base $\mathbf{w}=\left(w_{1}, \ldots, w_{g-t}\right)$ de $V \otimes_{K} \mathbb{C}_{v_{0}}$ (définie à la page 69 est orthonormée si $v_{0}$ est archimédienne, et qu'elle vérifie : pour tout $x=\sum_{j=1}^{g-t} x_{i} w_{j} \in V \otimes_{K} \mathbb{C}_{v_{0}},\|x\|_{v_{0}}>r_{p_{0}} \max _{j}\left|x_{j}\right|_{v_{0}}$ si $v_{0}$ est ultramétrique (voir page 60. En reprenant exactement les mêmes arguments qu'au paragraphe 8.8 (appliqués avec $D_{0}=0$ ), nous obtenons la proposition suivante.

Proposition 9.12. Il existe une constante $47 \geq 1$ telle que si $C_{0} \geq 47$, alors pour tout $\boldsymbol{\tau} \in \mathbb{N}^{g-t}$ tel que $|\boldsymbol{\tau}|=\ell$ et pour toute place $v \in \Sigma_{K^{\prime}}$ telle que $v \mid v_{0}$, on a

$$
\left|\prod_{i=1}^{n} \varphi_{i, \varepsilon_{i}}\left(m u_{i}\right)^{-D_{i}} \frac{1}{\boldsymbol{\tau} !} \mathcal{D}_{\mathbf{w}}^{\boldsymbol{\tau}} F_{s}(m u)\right|_{v} \leq e^{-U}\|s\|_{\alpha, v} .
$$

9.9. Conclusion. Nous allons obtenir une contradiction au moyen de la proposition suivante.

Proposition 9.13. Supposons que le réel $C_{0}$ est supérieur à $\max \left\{q_{46}^{2}, q 47\right\}$. Alors il existe une constante q48 telle que

$$
-{ }_{48} \frac{U}{C_{0} D} \leq h(\mathcal{J}) \leq \frac{2 q \overline{45} U}{D \sqrt{C_{0}}}-\frac{U}{2 D} .
$$


Démonstration. En reprenant les arguments du lemme 8.22 (en remplaçant $W$ par $V$ et $t_{G_{0} \times G}$ par $\left.t_{G}\right)$, on obtient

$$
h(\mathcal{J}) \geq-q_{48} \max \{1, h(V)\} \geq-q_{48} \frac{U}{C_{0} D} .
$$

D'après la proposition 9.12 et la définition de $\|\mathcal{J}\|_{v}$, on a

$$
\sum_{v \in \Sigma_{K^{\prime}}, v \mid v_{0}} \frac{\left[K_{v}^{\prime}: \mathbb{Q}_{v}\right]}{\left[K^{\prime}: \mathbb{Q}\right]} \log \|\mathcal{J}\|_{v} \leq \sum_{v \in \Sigma_{K^{\prime}}, v \mid v_{0}} \frac{\left[K_{v}^{\prime}: \mathbb{Q}_{v}\right]}{\left[K^{\prime}: \mathbb{Q}\right]} \log \|s\|_{\alpha, v}-\frac{U}{2 D}
$$

En appliquant la proposition 9.11, on obtient

$$
h(\mathcal{J}) \leq h_{\alpha}(s)-\frac{U}{2 D}+q 4 \frac{U}{D C_{0}} .
$$

Par ailleurs, rappelons que la section $s \in E \otimes_{K} K^{\prime}$ a été choisie de sorte que la majoration de la proposition 9.10 soit satisfaite :

$$
h_{\alpha}(s) \leq \frac{\square \lcm{45}}{D \sqrt{C_{0}}} U
$$

On en déduit la majoration voulue.

La proposition 9.13 implique que

$$
U \leq \frac{2 q 48+4 q 45}{\sqrt{C_{0}}} U .
$$

On a donc une contradiction si $C_{0}>\max \left\{q_{40}, q_{41}, q_{46,}^{2} q_{47},\left(2 q_{48}+44_{45}\right)^{2}\right\}$, et alors

$$
\log d(u, V)>-2 \sqrt{C_{0}} U .
$$

Pour conclure, il suffit de majorer le terme $U$ par les quantités $U_{0}$ et $U_{1}$ des théorèmes 4.1 et 4.2 (à constantes près). Ces comparaisons sont analogues à celles du paragraphe 8.11 et ne font pas intervenir d'argument nouveau.

\section{Références}

[1] Y. Amice, Interpolation p-adique, dans : Les Tendances Géométriques en Algèbre et Théorie des Nombres, Éditions du Centre National de la Recherche Scientifique, Paris, 1966, 15-25.

[2] D. Bertrand, Lemmes de Schwarz et lemmes d'approximations dans les domaines ultramétriques, Groupe d'Étude d'Analyse Ultramétrique (3e année : 1975/76), no. 2 (MarseilleLuminy, 1976), exp. J8, 12 pp., 1977.

[3] D. Bertrand et P. Philippon, Sous-groupes algébriques de groupes algébriques commutatifs, Illinois J. Math. 32 (1988), 263-280.

[4] E. Bombieri and J. Vaaler, On Siegel's lemma, Invent. Math. 73 (1983), 11-32.

[5] S. Bosch, W. Lütkebohmert and M. Raynaud, Néron Models, Ergeb. Math. Grenzgeb. 21, Springer, Berlin, 1990.

[6] V. Bosser et E. Gaudron, Logarithmes des points rationnels des variétés abéliennes, Canad. J. Math. 71 (2019), 247-298.

[7] J.-B. Bost, Périodes et isogénies des variétés abéliennes sur les corps de nombres (d'après D. Masser et G. Wüstholz), dans : Séminaire Bourbaki, Vol. 1994/95, exp. 795, Astérisque 237 (1996), 4, 115-161. 
[8] J.-B. Bost, Algebraic leaves of algebraic foliations over number fields, Publ. Math. Inst. Hautes Études Sci. 93 (2001), 161-221.

[9] N. Bourbaki, Éléments de mathématique. Fasc. XXXVII. Groupes et algèbres de Lie. Chapitre II: Algèbres de Lie libres. Chapitre III: Groupes de Lie, Act. Sci. Industr. 1349, Hermann, Paris, 1972.

[10] M. Brion, Some structure theorems for algebraic groups, dans : Proc. Sympos. Pure Math. 94, Amer. Math. Soc., 2017, 53-125.

[11] M. Chardin, Une majoration de la fonction de Hilbert et ses conséquences pour l'interpolation algébrique, Bull. Soc. Math. France 117 (1989), 305-318.

[12] P. L. Cijsouw and M. Waldschmidt, Linear forms and simultaneous approximations, Compos. Math. 34 (1977), 173-197.

[13] B. Conrad, A modern proof of Chevalley's theorem on algebraic groups, J. Ramanujan Math. Soc. 17 (2002), 1-18.

[14] S. David, Minorations de formes linéaires de logarithmes elliptiques, Mém. Soc. Math. France (N.S.) 62 (1995), iv+143 pp.

[15] S. David and N. Hirata-Kohno, Linear forms in elliptic logarithms, J. Reine Angew. Math. 628 (2009), 37-89.

[16] M. Demazure, Fibrés tangents, algèbres de Lie, dans : Schémas en Groupes (Sém. Géométrie Algébrique, Inst. Hautes Études Sci., 1963), fasc. 1, exp. 2, 40 pp., Inst. Hautes Études Sci., Paris, 1963.

[17] N. I. Fel'dman, An improvement of the estimate of a linear form in the logarithms of algebraic numbers, Mat. Sb. (N.S.) 77 (119) (1968), 423-436 (en russe).

[18] É. Gaudron, Mesure d'indépendance de logarithmes dans un groupe algébrique commutatif, Thèse de doctorat, Univ. Jean Monnet, Saint Étienne, 2001.

[19] É. Gaudron, Mesures d'indépendance linéaire de logarithmes dans un groupe algébrique commutatif, Invent. Math. 162 (2005), 137-188.

[20] É. Gaudron, Formes linéaires de logarithmes effectives sur les variétés abéliennes, Ann. Sci. École Norm. Sup. (4) 39 (2006), 699-773.

[21] É. Gaudron, Étude du cas rationnel de la théorie des formes linéaires de logarithmes, J. Number Theory 127 (2007), 220-261.

[22] É. Gaudron, Pentes des fibrés vectoriels adéliques sur un corps global, Rend. Sem. Mat. Univ. Padova 119 (2008), 21-95.

[23] É. Gaudron, Géométrie des nombres adélique et lemmes de Siegel généralisés, Manuscripta Math. 130 (2009), 159-182.

[24] É. Gaudron, Minorations simultanées de formes linéaires de logarithmes de nombres algébriques, Bull. Soc. Math. France 142 (2014), 1-62.

[25] É. Gaudron et G. Rémond, Lemmes de Siegel d'évitement, Acta Arith. 154 (2012), 125-136.

[26] É. Gaudron et G. Rémond, Minima, pentes et algèbre tensorielle, Israel J. Math. 195 (2013), 565-591.

[27] É. Gaudron et G. Rémond, Corps de Siegel, J. Reine Angew. Math. 726 (2017), 187-247.

[28] N. Hirata-Kohno, Formes linéaires de logarithmes de points algébriques sur les groupes algébriques, Invent. Math. 104 (1991), 401-433.

[29] N. Hirata-Kohno, Approximations simultanées sur les groupes algébriques commutatifs, Compos. Math. 86 (1993), 69-96.

[30] H. Lange, A remark on the degrees of commutative algebraic groups, Illinois J. Math. 33 (1989), 409-415. 
[31] E. M. Matveev, Arithmetic properties of the values of generalized binomials, Mat. Zametki 54 (1993), no. 4, 76-81, 159 (en russe).

[32] P. Philippon, Lemmes de zéros dans les groupes algébriques commutatifs, Bull. Soc. Math. France 114 (1986), 355-383.

[33] P. Philippon, Nouveaux lemmes de zéros dans les groupes algébriques commutatifs, Rocky Mountain J. Math. 26 (1996), 1069-1088.

[34] P. Philippon et M. Waldschmidt, Formes linéaires de logarithmes simultanées sur les groupes algébriques commutatifs, dans : Séminaire de Théorie des Nombres, Paris 1986-87, Progr. Math. 75, Birkhäuser, Boston, MA, 1988, 313-347.

[35] P. Philippon et M. Waldschmidt, Formes linéaires de logarithmes sur les groupes algébriques commutatifs, Illinois J. Math. 32 (1988), 281-314.

[36] P. Robba, Lemmes de Schwarz et lemmes d'approximations p-adiques en plusieurs variables, Invent. Math. 48 (1978), 245-277.

[37] D. Roy and J. L. Thunder: An absolute Siegel's lemma, J. Reine Angew. Math. 476 (1996), $1-26$.

[38] J.-P. Serre, Lie Algebras and Lie Groups, W. A. Benjamin, New York, 1965.

[39] M. Waldschmidt, Nombres transcendants et groupes algébriques, Astérisque 69 (1979).

[40] M. Waldschmidt, A lower bound for linear forms in logarithms, Acta Arith. 37 (1980), $257-283$.

[41] M. Waldschmidt, Approximation diophantienne dans les groupes algébriques commutatifs. I. Une version effective du théorème du sous-groupe algébrique, J. Reine Angew. Math. 493 (1997), 61-113.

[42] U. Zannier, Lecture Notes on Diophantine Analysis, Edizioni della Normale, Pisa, 2009.

[43] S. Zhang, Positive line bundles on arithmetic varieties, J. Amer. Math. Soc. 8 (1995), 187-221. 